\title{
Enantioselective Synthesis of Quaternary Stereocenters via Chromium Catalysis
}

\section{SUPPORTING INFORMATION}

Yang Xiong, Guozhu Zhang

State Key Laboratory of Organometallic Chemistry, Shanghai Institute of Organic Chemistry, Chinese Academy of Sciences, 345 Lingling Road, Shanghai 200032, P. R. China

\section{CONTENTS}

1. General Information $\quad$ S2

2. Experimental Details S3

(1) Preparation and Characterization of Allylic Chlorides S3

(2) Preparation and Characterization of Ligands S3

$\begin{array}{ll}\text { (3) General Procedure } & \text { S7 }\end{array}$

(4) Evaluation of Chiral Ligands and Other Reaction Parameters S8

(5) The Characterization of Products $\quad \mathbf{S 1 0}$

$\begin{array}{ll}\text { (6) Reference } & \mathbf{S 1 9}\end{array}$

3. Copies of NMR Spectra and HPLC Chromatographs $\mathbf{S 2 0}$ 


\section{General Information}

NMR spectra were recorded at room temperature on the following spectrometers: Agilent (400 MHz) and VARIAN (400 MHz). Chemical shifts are given in ppm and coupling constants in $\mathrm{Hz}$. ${ }^{1} \mathrm{H}$ spectra were calibrated in relation to the reference measurement of TMS $(0.00 \mathrm{ppm}) .{ }^{13} \mathrm{C}$ spectra were calibrated in relation to deuterated solvents, namely $\mathrm{CDCl}_{3}(77.16 \mathrm{ppm})$. The following abbreviations were used for ${ }^{1} \mathrm{H}$ NMR spectra to indicate the signal multiplicity: $s$ (singlet), $d$ (doublet), $t$ (triplet), $q$ (quartet) and $m$ (multiplet) as well as combinations of them. When combinations of multiplicities are given the first character noted refers to the largest coupling constant. High performance liquid chromatography (HPLC) was carried out with Agilent 1260 Infinity on a UV spectrophotometric detector (210 $\mathrm{nm}$, Agilent). For ESI ${ }^{+}$-spectra an El'HR (GC-TOF) spectrometer was applied. Infrared Spectroscopy (IR) was processed on an FT-IR spectrometer named Nicolet 380 . The method is denoted in brackets. For the most significant bands the wave number $\tilde{v}\left(\mathrm{~cm}^{-1}\right)$ is given.

Chemicals were purchased from commercial suppliers. Unless stated otherwise, all the substrates and solvents were purified and dried according to standard methods prior to use. Reactions requiring inert conditions were carried out in glove box. 


\section{Experimental details}

\section{(1) Preparation and Characterization of Allylic Chlorides}

Allylic chlorides 1a - 1f were synthesized according to previously described literature methods ${ }^{[1 \mathrm{a}, \mathrm{b}]}$ using ethyl acetate for extraction instead of diethyl ether; Allylic chlorides $\mathbf{1 g}$ was synthesized according to previously described literature methods ${ }^{[1 \mathrm{c}]}$.

\section{(2) Preparation and Characterization of Ligands}

\section{General Procedure A: Preparation of Chiral Sulfonamide Ligands}

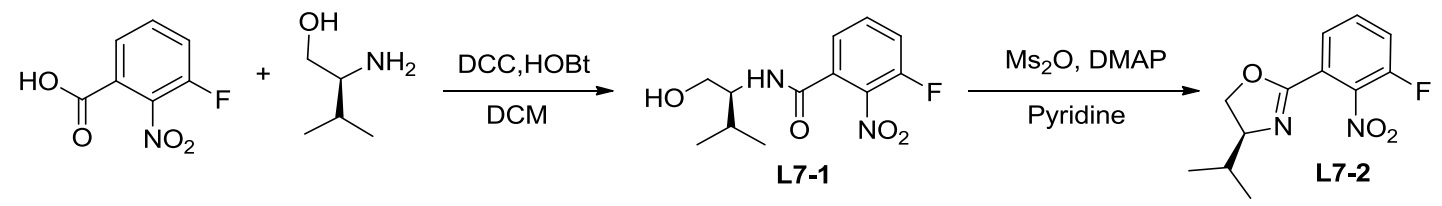

\section{Synthesis of L7-1}

To a $250 \mathrm{~mL}$ round bottom flask was added $10 \mathrm{~g}$ of 3-fluoro-2-nitrobenzoic acid $(54.0 \mathrm{mmol}$, 1 equiv.), $400 \mathrm{mg}$ of 1 -hydroxybenzotriazole ( $3 \mathrm{mmol}, 0.015$ equiv.), $100 \mathrm{~mL}$ of methylene chloride, $7.78 \mathrm{~g}$ of (S)-2-amino-3-methylbutan-1-ol (75.6 mmol, 1.4 equiv.),, and, after cooling to $0 \stackrel{\circ}{\circ}, 22.2 \mathrm{~g}$ of dicyclohexylcarbodiimide (108 mmol, 2 equiv.). The reaction was allowed to warm to room temperature overnight $(12 \mathrm{~h})$. The reaction mixture was filtered through celite which was then washed with additional methylene chloride. The combined organic phases were concentrated in vacuo. The product was separated by flash column chromatography (Petroleum ether : Ethyl acetate $=1: 1$ ) as yellow/white solid $(12.4 \mathrm{~g}, 45.9$ $\mathrm{mmol})$ in $85 \%$ yield. ${ }^{1} \mathrm{H} \mathrm{NMR}\left(400 \mathrm{MHz}, \mathrm{CDCl}_{3}\right) \delta 7.60-7.51(\mathrm{~m}, 1 \mathrm{H}), 7.39(\mathrm{~d}, J=7.7 \mathrm{~Hz}$, $1 \mathrm{H}), 7.37-7.30(\mathrm{~m}, 1 \mathrm{H}), 6.35(\mathrm{~d}, J=8.5 \mathrm{~Hz}, 1 \mathrm{H}), 3.91-3.82(\mathrm{~m}, 1 \mathrm{H}), 3.78(\mathrm{~d}, J=0.8 \mathrm{~Hz}$, 2H), $2.18(\mathrm{~s}, 1 \mathrm{H}), 1.96(\mathrm{dd}, J=14.0,6.9 \mathrm{~Hz}, 1 \mathrm{H}), 1.00$ (dd, $J=6.8,3.5 \mathrm{~Hz}, 6 \mathrm{H}) .{ }^{13} \mathrm{C}$ NMR $\left(101 \mathrm{MHz}, \mathrm{CDCl}_{3}\right) \delta 164.4,132.9,132.8,123.51,123.5,119.4,119.2,62.9,57.6,29.1,19.5$, 18.9. ${ }^{19} \mathrm{~F} \mathrm{NMR}\left(376 \mathrm{MHz}, \mathrm{CDCl}_{3}\right) \delta-121.00,-121.01,-121.02,-121.02,-121.03,-121.03$, -121.04, -121.05. HRMS (El $\left.{ }^{(+)}, 70 \mathrm{eV}\right): \mathrm{C}_{12} \mathrm{H}_{13} \mathrm{~N}_{2} \mathrm{O}_{3} \mathrm{~F}\left[\mathrm{M}-\mathrm{H}_{2} \mathrm{O}\right]^{+}$: calcd: 252.0913, found: 252.0910 .

\section{Synthesis of L7-2}

To a $100 \mathrm{~mL}$ round bottom flask was added $3.6 \mathrm{~g}$ (13.2 mmol, 1 equiv. ) of the above white solid (S)-3-fluoro-N-(1-hydroxy-3-methylbutan-2-yl)-2-nitrobenzamide, $30 \mathrm{~mL}$ of methylene chloride and $6 \mathrm{~mL}$ of pyridine, after cooling to $0 \stackrel{\circ}{\circ}$, $3.4 \mathrm{~g}$ (19.8 mmol, 1.5 equiv.) of $\mathrm{Ms}_{2} \mathrm{O}$. The reaction was warm to room temperature, after 30 mins, heated at reflux under nitrogen $8 \mathrm{~h}$. The organic layer was washed with water and dried over anhydrous sodium sulfate. The L7-2 (S)-2-(3-fluoro-2-nitrophenyl)-4-isopropyl-4,5-dihydroox-azole (3.0 g, 11.9 mmol) was purified by flash column chromatography (Petroleum ether : Ethyl acetate $=1: 1$ ), in $90 \%$ yield. ${ }^{1} \mathrm{H}$ NMR $\left(400 \mathrm{MHz}, \mathrm{CDCl}_{3}\right) \delta 7.72$ (dt, $\left.J=7.9,1.1 \mathrm{~Hz}, 1 \mathrm{H}\right), 7.50(\mathrm{td}, J=8.2,5.2 \mathrm{~Hz}$, $1 \mathrm{H}), 7.34(\mathrm{td}, J=9.1,1.2 \mathrm{~Hz}, 1 \mathrm{H}), 4.40-4.31(\mathrm{~m}, 1 \mathrm{H}), 4.16-4.02(\mathrm{~m}, 2 \mathrm{H}), 1.86-1.72(\mathrm{~m}$, $1 \mathrm{H}), 0.95(\mathrm{~d}, J=6.8 \mathrm{~Hz}, 3 \mathrm{H}), 0.89(\mathrm{~d}, J=6.8 \mathrm{~Hz}, 3 \mathrm{H}) .{ }^{13} \mathrm{C} \mathrm{NMR}\left(101 \mathrm{MHz}, \mathrm{CDCl}_{3}\right) \delta 158.2$, 158.2, 154.7, 152.2, 131.5, 131.4, 125.7, 125.6, 122.4, 119.6, 119.4, 73.0, 71.0, 32.7, 18.5, 18.2. ${ }^{19} \mathrm{~F}$ NMR (376 MHz, $\left.\mathrm{CDCl}_{3}\right) \delta-124.08,-124.10,-124.11,-124.12 . \mathrm{HRMS}\left(\mathrm{El}^{(+)}, 70\right.$ $\mathrm{eV}): \mathrm{C}_{12} \mathrm{H}_{13} \mathrm{~N}_{2} \mathrm{O}_{3} \mathrm{~F}[\mathrm{M}]^{+}$: calcd. 252.0920, found: 252.0910 . 


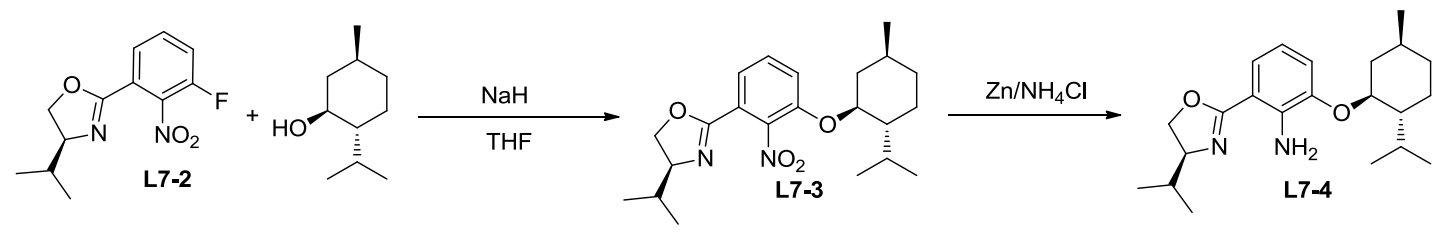

\section{Synthesis of L7-3}

To a $100 \mathrm{~mL}$ round bottom flask was added a slurry of $5.1 \mathrm{~g}$ of $\mathrm{NaH}(18 \mathrm{mmol}, 60 \%$ in mineral oil, 1.8 equiv.) in anhydrous THF (35 mL) was added $1.56 \mathrm{~g}$ of unnatural menthol (1S,2R,5S)-2-isopropyl-5-methylcyclohexan-1-ol (14 mmol, 1.4 equiv.) at $0{ }^{\circ} \mathrm{C}$ with stirring for $1 \mathrm{~h}$. To the cooled reaction mixture was slowly added $2.53 \mathrm{~g}$ of L7-2 $(10 \mathrm{mmol}, 1.0$ equiv.) via syringe. The reaction was allowed to warm to room temperature and stirred for 6 $\mathrm{h}$, quenched with water at $0{ }^{\circ} \mathrm{C}$. The organic solvent (THF) was removed in vacuo and the slurry was extracted with ethyl acetate. The combined organic layers were washed with brine, water, dried over $\mathrm{Na}_{2} \mathrm{SO}_{4}$ and filtered. The solvent was removed in vacuo to afford crude L7-3, which was used for the next step without further purification. ${ }^{1} \mathrm{H} N \mathrm{NM}(400 \mathrm{MHz}$, $\left.\mathrm{CDCl}_{3}\right) \delta 7.46(\mathrm{~d}, J=7.1 \mathrm{~Hz}, 1 \mathrm{H}), 7.38(\mathrm{t}, J=8.1 \mathrm{~Hz}, 1 \mathrm{H}), 7.15(\mathrm{~d}, J=8.2 \mathrm{~Hz}, 1 \mathrm{H}), 4.40-$ $4.30(\mathrm{~m}, 1 \mathrm{H}), 4.09$ (dd, $J=3.7,2.2 \mathrm{~Hz}, 2 \mathrm{H}), 3.39(\mathrm{td}, J=10.4,4.3 \mathrm{~Hz}, 1 \mathrm{H}), 2.24-2.01(\mathrm{~m}$, $2 \mathrm{H}$ ), 1.81 (dq, $J=13.2,6.7 \mathrm{~Hz}, 1 \mathrm{H}), 1.70$ (tdd, $J=8.6,6.0,3.1 \mathrm{~Hz}, 2 \mathrm{H}), 1.58-1.36(\mathrm{~m}, 3 \mathrm{H})$, $0.97(\mathrm{~d}, J=6.8 \mathrm{~Hz}, 4 \mathrm{H}), 0.93-0.86(\mathrm{~m}, 12 \mathrm{H}), 0.72(\mathrm{~d}, J=7.0 \mathrm{~Hz}, 3 \mathrm{H}) \cdot{ }^{13} \mathrm{C}$ NMR $(101 \mathrm{MHz}$, $\left.\mathrm{CDCl}_{3}\right) \delta 159.2,149.7,130.4,121.4,121.0,117.1,79.7,72.7,70.7,47.5,39.6,34.1,32.7$, $31.4,25.7,23.43,22.0,20.7,18.6,18.1,16.3 . \mathrm{HRMS}\left(\mathrm{El}^{(+)}, 70 \mathrm{eV}\right): \mathrm{C}_{22} \mathrm{H}_{34} \mathrm{~N}_{2} \mathrm{O}_{2}[\mathrm{M}]^{+}$: calcd: 358.2609, found: 358.2620 .

\section{Synthesis of L7-4}

To a $100 \mathrm{~mL}$ round bottom flask was added $1.94 \mathrm{~g}$ of $\mathbf{L 7 - 3}(5 \mathrm{mmol}, 1.0$ equiv.) in $30 \mathrm{ml}$ acetone, $1.3 \mathrm{~g}$ of zinc powder (20 mmol, 4.0 equiv.) and $3.3 \mathrm{~mL}$ of $6 \mathrm{~mol} / \mathrm{L}$ ammonium chloride (20 mmol, equiv.). The mixture was vigorously shaken at room temperature for $4 \mathrm{~h}$. The filtrate was diluted with EtOAc and extracted saturated aqueous $\mathrm{NaHCO}_{3}$. The organic layer was collected, dried $\mathrm{Na}_{2} \mathrm{SO}_{4}$, and concentrated to afford L7-4 $(1.76 \mathrm{~g}, 4.9 \mathrm{mmol})$ in $98 \%$ yield $^{[2]} .{ }^{1} \mathrm{H}$ NMR $\left(400 \mathrm{MHz}, \mathrm{CDCl}_{3}\right) \delta 7.39-7.18(\mathrm{~m}, 1 \mathrm{H}), 6.82(\mathrm{~d}, \mathrm{~J}=7.6 \mathrm{~Hz}, 1 \mathrm{H})$, $6.56(\mathrm{t}, \mathrm{J}=8.0 \mathrm{~Hz}, 1 \mathrm{H}), 6.38(\mathrm{~s}, 2 \mathrm{H}), 4.38-4.26(\mathrm{~m}, 1 \mathrm{H}), 4.08(\mathrm{ddd}, \mathrm{J}=14.3,12.8,5.9 \mathrm{~Hz}$, $2 \mathrm{H}), 3.99(\mathrm{t}, \mathrm{J}=8.0 \mathrm{~Hz}, 1 \mathrm{H}), 2.32-2.11(\mathrm{~m}, 2 \mathrm{H}), 1.83-1.52(\mathrm{~m}, 3 \mathrm{H}), 1.49-1.38(\mathrm{~m}, 3 \mathrm{H})$, $1.26(\mathrm{~d}, \mathrm{~J}=7.1 \mathrm{~Hz}, 1 \mathrm{H}), 1.18-1.08(\mathrm{~m}, 1 \mathrm{H}), 1.03(\mathrm{~d}, \mathrm{~J}=6.7 \mathrm{~Hz}, 3 \mathrm{H}), 0.92(\mathrm{dd}, \mathrm{J}=10.8$, $6.9 \mathrm{~Hz}, 9 \mathrm{H}), 0.78(\mathrm{~d}, \mathrm{~J}=6.9 \mathrm{~Hz}, 3 \mathrm{H}) .{ }^{13} \mathrm{C} \operatorname{NMR}\left(101 \mathrm{MHz}, \mathrm{CDCl}_{3}\right) \delta 163.7,144.9,140.5$, 120.8, 114.5, 113.7, 108.7, 72.9, 68.8, 48.2, 40.3, 34.5, 33.3, 31.4, 26.2, 23.7, 22.2, 20.8, 19.0, 18.78, 16.8 .

\section{Synthesis of Chiral Sulfonamide Ligand L7}

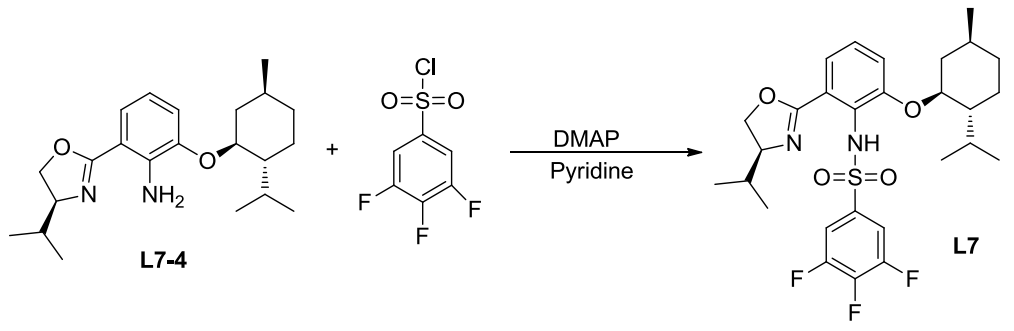


To a $100 \mathrm{~mL}$ round bottom flask was added $0.880 \mathrm{~g}$ of $\mathbf{L 7 - 4}(2.5 \mathrm{mmol}, 1.0$ equiv.) in anhydrous pyridine (15 mL), $1.15 \mathrm{~g}$ of 3,4,5-trifluorobenzenesulfonyl chloride (5 mmol, 2.0 equiv.) and $66 \mathrm{mg}$ of DMAP ( $1 \mathrm{mmol}, 0.2$ equiv.). The solution was stirred overnight before quenched with water. The mixture was extracted with ethyl acetate and the combined organic layers were washed with $1 \mathrm{NHCl}$, brine and water. The organic layer was dried over $\mathrm{Na}_{2} \mathrm{SO}_{4}$ and filtered. The crude product purified by chromatography (Petroleum ether : Ethyl acetate $=10: 1)$ to afford pure product $\mathbf{L} 7(1.11 \mathrm{~g}, 2.0 \mathrm{mmol})$, in $80 \%$ yield. ${ }^{1} \mathrm{H}$ NMR $\left(400 \mathrm{MHz}, \mathrm{CDCl}_{3}\right) \delta 12.09(\mathrm{~s}, 1 \mathrm{H}), 7.59(\mathrm{t}, \mathrm{J}=6.4 \mathrm{~Hz}, 2 \mathrm{H}), 7.34(\mathrm{~d}, \mathrm{~J}=6.9 \mathrm{~Hz}, 1 \mathrm{H}), 7.06(\mathrm{t}$, $\mathrm{J}=8.0 \mathrm{~Hz}, 1 \mathrm{H}), 6.96(\mathrm{~d}, \mathrm{~J}=7.9 \mathrm{~Hz}, 1 \mathrm{H}), 4.37(\mathrm{~s}, 1 \mathrm{H}), 4.09(\mathrm{~d}, \mathrm{~J}=12.1 \mathrm{~Hz}, 2 \mathrm{H}), 3.88(\mathrm{td}, \mathrm{J}$ $=10.7,4.1 \mathrm{~Hz}, 1 \mathrm{H}), 1.94(\mathrm{~d}, \mathrm{~J}=12.2 \mathrm{~Hz}, 1 \mathrm{H}), 1.80(\mathrm{~m}, 1 \mathrm{H}), 1.63(\mathrm{ddd}, \mathrm{J}=22.8,13.2,8.0$ $\mathrm{Hz}, 2 \mathrm{H}), 1.48(\mathrm{dd}, \mathrm{J}=13.3,3.3 \mathrm{~Hz}, 1 \mathrm{H}), 1.36-1.25(\mathrm{~m}, 2 \mathrm{H}), 1.20(\mathrm{~d}, \mathrm{~J}=12.7 \mathrm{~Hz}, 1 \mathrm{H}), 1.03$ $(\mathrm{d}, \mathrm{J}=6.7 \mathrm{~Hz}, 3 \mathrm{H}), 0.91(\mathrm{t}, \mathrm{J}=9.0 \mathrm{~Hz}, 3 \mathrm{H}), 0.84(\mathrm{dd}, \mathrm{J}=10.6,6.3 \mathrm{~Hz}, 3 \mathrm{H}), 0.77-0.67(\mathrm{~m}$, $2 \mathrm{H}), 0.63(\mathrm{~d}, \mathrm{~J}=7.0 \mathrm{~Hz}, 3 \mathrm{H}), 0.46(\mathrm{~d}, \mathrm{~J}=6.9 \mathrm{~Hz}, 3 \mathrm{H}), 0.42-0.26(\mathrm{~m}, 1 \mathrm{H}) .{ }^{13} \mathrm{C}$ NMR $(101$ $\left.\mathrm{MHz}, \mathrm{CDCl}_{3}\right) \delta 151.4,128.5,125.3,120.8,119.5,116.3,112.1,112.1,111.9,78.8,72.6$, 70.0, 47.3, 39.8, 34.38, 33.2, 31.5, 25.3, 22.7, 22.1, 20.6, 18.7, 18.7, 15.6. ${ }^{19} \mathrm{~F}$ NMR (376

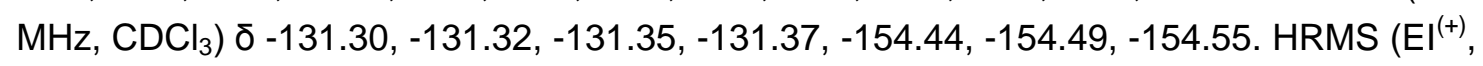
$70 \mathrm{eV}): \mathrm{C}_{28} \mathrm{H}_{36} \mathrm{O}_{4} \mathrm{~N}_{2} \mathrm{~F}_{3} \mathrm{~S}[\mathrm{M}+1]^{+}$: calcd. 553.2343, found: 553.2342. [ $\left.\alpha\right]_{\mathrm{D}}{ }^{20}=21.8(\mathrm{c}=0.60$, $\left.\mathrm{CH}_{2} \mathrm{Cl}_{2}\right) ; \mathrm{mp}=95^{\circ} \mathrm{C}$.

Synthesis of N-(2-((S)-4-isopropyl-4,5-dihydrooxazol-2-yl)-6-(((1S,2R,5S)-2-isopropyl5-methylcyclohexyl)oxy)phenyl)benzenesulfonamide (L6)

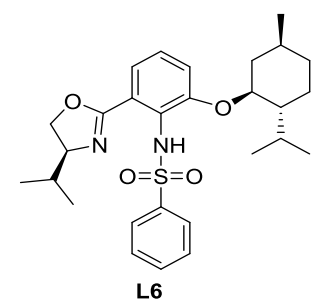

According to the general procedure A, L6 $(199.5 \mathrm{mg}, 0.4 \mathrm{mmol})$ was prepared from L7-4 (179.3 $\mathrm{mg}, 0.5 \mathrm{mmol})$, benzenesulfonyl chloride(176.6 $\mathrm{mg}, 1 \mathrm{mmol}$ ) as white solid in $80 \%$ yield $^{[2]}$.

${ }^{1} \mathrm{H} \mathrm{NMR}\left(400 \mathrm{MHz}, \mathrm{CDCl}_{3}\right) \delta 11.82(\mathrm{~s}, 1 \mathrm{H}), 7.98(\mathrm{~d}, \mathrm{~J}=7.3 \mathrm{~Hz}, 2 \mathrm{H}), 7.53(\mathrm{~d}, \mathrm{~J}=7.2 \mathrm{~Hz}, 1 \mathrm{H})$, $7.47(\mathrm{t}, \mathrm{J}=7.4 \mathrm{~Hz}, 2 \mathrm{H}), 7.37(\mathrm{~d}, \mathrm{~J}=7.7 \mathrm{~Hz}, 1 \mathrm{H}), 7.08(\mathrm{t}, \mathrm{J}=8.0 \mathrm{~Hz}, 1 \mathrm{H}), 7.00(\mathrm{~d}, \mathrm{~J}=8.1 \mathrm{~Hz}$, $1 \mathrm{H}), 4.37(\mathrm{~d}, \mathrm{~J}=7.3 \mathrm{~Hz}, 1 \mathrm{H}), 4.21-4.02(\mathrm{~m}, 2 \mathrm{H}), 3.89(\mathrm{~d}, \mathrm{~J}=4.1 \mathrm{~Hz}, 1 \mathrm{H}), 1.92(\mathrm{~d}, \mathrm{~J}=12.4$ $\mathrm{Hz}, 1 \mathrm{H}), 1.88-1.68(\mathrm{~m}, 2 \mathrm{H}), 1.57(\mathrm{~d}, \mathrm{~J}=12.2 \mathrm{~Hz}, 1 \mathrm{H}), 1.50-1.40(\mathrm{~m}, 1 \mathrm{H}), 1.28(\mathrm{~d}, \mathrm{~J}=5.8$ $\mathrm{Hz}, 2 \mathrm{H}), 1.08(\mathrm{~d}, \mathrm{~J}=6.7 \mathrm{~Hz}, 3 \mathrm{H}), 0.98(\mathrm{~d}, \mathrm{~J}=6.7 \mathrm{~Hz}, 3 \mathrm{H}), 0.85(\mathrm{~d}, \mathrm{~J}=6.5 \mathrm{~Hz}, 3 \mathrm{H}), 0.82-$ $0.74(\mathrm{~m}, 2 \mathrm{H}), 0.65(\mathrm{~d}, \mathrm{~J}=7.0 \mathrm{~Hz}, 3 \mathrm{H}), 0.51(\mathrm{~d}, \mathrm{~J}=6.9 \mathrm{~Hz}, 3 \mathrm{H}), 0.42-0.26(\mathrm{~m}, 1 \mathrm{H})$.

Synthesis of 3,5-difluoro-N-(2-((S)-4-isopropyl-4,5-dihydrooxazol-2-yl)-6-(((1S,2R,5S)2-iso-propyl-5-methylcyclohexyl)oxy)phenyl)benzenesulfonamide (L8)

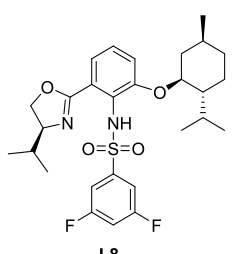


According to the general procedure A, $\mathbf{L} 8(219.2 \mathrm{mg}, 0.41 \mathrm{mmol})$ was prepared from $\mathbf{~} \mathbf{7 - 4}$ (219.2 $\mathrm{mg}, 0.5 \mathrm{mmol}), 3,5$-difluorobenzenesulfonyl chloride $(212.6 \mathrm{mg}, 1 \mathrm{mmol}$ ) as white solid in $82 \%$.

${ }^{1} \mathrm{H}$ NMR $\left(400 \mathrm{MHz}, \mathrm{CDCl}_{3}\right) \delta 12.17(\mathrm{~s}, 1 \mathrm{H}), 7.55(\mathrm{~d}, \mathrm{~J}=4.5 \mathrm{~Hz}, 2 \mathrm{H}), 7.40(\mathrm{~d}, \mathrm{~J}=7.7 \mathrm{~Hz}$, $1 \mathrm{H}), 7.12(\mathrm{t}, \mathrm{J}=8.0 \mathrm{~Hz}, 1 \mathrm{H}), 7.01(\mathrm{dd}, \mathrm{J}=14.6,8.3 \mathrm{~Hz}, 2 \mathrm{H}), 4.42(\mathrm{t}, \mathrm{J}=7.3 \mathrm{~Hz}, 1 \mathrm{H}), 4.24-$ $4.03(\mathrm{~m}, 2 \mathrm{H}), 3.94(\mathrm{td}, \mathrm{J}=10.8,4.1 \mathrm{~Hz}, 1 \mathrm{H}), 2.17-1.44(\mathrm{~m}, 5 \mathrm{H}), 1.29(\mathrm{~d}, \mathrm{~J}=30.0 \mathrm{~Hz}, 2 \mathrm{H})$, $1.10(\mathrm{~d}, \mathrm{~J}=6.6 \mathrm{~Hz}, 3 \mathrm{H}), 0.99(\mathrm{~d}, \mathrm{~J}=6.7 \mathrm{~Hz}, 3 \mathrm{H}), 0.88(\mathrm{~d}, \mathrm{~J}=6.5 \mathrm{~Hz}, 3 \mathrm{H}), 0.80(\mathrm{~m}, 2 \mathrm{H})$, $0.69(\mathrm{~d}, \mathrm{~J}=7.0 \mathrm{~Hz}, 3 \mathrm{H}), 0.52(\mathrm{~d}, \mathrm{~J}=6.9 \mathrm{~Hz}, 3 \mathrm{H}), 0.35(\mathrm{t}, \mathrm{J}=10.8 \mathrm{~Hz}, 1 \mathrm{H}) .{ }^{13} \mathrm{C} \mathrm{NMR}(101$ $\left.\mathrm{MHz}, \mathrm{CDCl}_{3}\right) \delta 163.7,163.6,161.2,151.4,125.1,120.7,119.4,116.2,110.6,110.3,107.4$, 107.1, 106.9, 78.7, 72.6, 70.0, 46.8, 39.6, 34.3, 33.2, 31.5, 25.3, 22.7, 22.1, 20.6, 18.7, 15.6. HRMS $\left(\mathrm{El}^{(+)}, 70 \mathrm{eV}\right): \mathrm{C}_{28} \mathrm{H}_{37} \mathrm{O}_{4} \mathrm{~N}_{2} \mathrm{~F}_{2} \mathrm{~S}[\mathrm{M}+1]^{+}$: calcd: 535.2437, found: 535.2437. $[\alpha]_{D}^{20}=19.1\left(\mathrm{c}=0.49, \mathrm{CH}_{2} \mathrm{Cl}_{2}\right) ; \mathrm{mp}=116{ }^{\circ} \mathrm{C}$.

Synthesis of 2,3,5,6-tetrafluoro-N-(2-((S)-4-isopropyl-4,5-dihydrooxazol-2-yl)-6 -(((1S,2R,5S)-2-isopropyl-5-methylcyclohexyl)oxy)phenyl)benzenesulfonamide (L9)<smiles>CC1CCC(C(C)C)C(Oc2cccc(C3=NC(C(C)C)CO3)c2NS(=O)(=O)c2c(F)c(F)c(F)c(F)c2F)C1</smiles>

According to the general procedure A, $\mathbf{L} \mathbf{9}(111.3 \mathrm{mg}, 0.2 \mathrm{mmol})$ was prepared from L7-4 (89.6 mg, $0.25 \mathrm{mmol}$ ), 2,3,5,6-tetrafluorobenzenesulfonyl chloride (124.3 mg, $0.5 \mathrm{mmol}$ ) as white solid in $78 \%$ yield .

${ }^{1} \mathrm{H}$ NMR $\left(400 \mathrm{MHz}, \mathrm{CDCl}_{3}\right) \delta 12.50(\mathrm{~s}, 1 \mathrm{H}), 7.41$ (dd, $\left.\mathrm{J}=7.7,0.9 \mathrm{~Hz}, 1 \mathrm{H}\right), 7.28$ (dd, J = 11.3, $4.1 \mathrm{~Hz}, 1 \mathrm{H}), 7.13(\mathrm{t}, \mathrm{J}=8.0 \mathrm{~Hz}, 1 \mathrm{H}), 7.06(\mathrm{~d}, \mathrm{~J}=7.7 \mathrm{~Hz}, 1 \mathrm{H}), 4.44(\mathrm{t}, \mathrm{J}=7.8 \mathrm{~Hz}, 1 \mathrm{H}), 4.24-$ $4.07(\mathrm{~m}, 2 \mathrm{H}), 3.97(\mathrm{td}, \mathrm{J}=10.7,4.2 \mathrm{~Hz}, 1 \mathrm{H}), 2.06(\mathrm{~d}, \mathrm{~J}=12.0 \mathrm{~Hz}, 1 \mathrm{H}), 1.86(\mathrm{dd}, \mathrm{J}=13.3$, $6.6 \mathrm{~Hz}, 1 \mathrm{H}), 1.70(\mathrm{~m}, 3 \mathrm{H}), 1.53(\mathrm{dd}, \mathrm{J}=13.4,3.3 \mathrm{~Hz}, 1 \mathrm{H}), 1.45-1.30(\mathrm{~m}, 1 \mathrm{H}), 1.11(\mathrm{~d}, \mathrm{~J}=$ $6.7 \mathrm{~Hz}, 3 \mathrm{H}), 1.00(\mathrm{~d}, \mathrm{~J}=6.7 \mathrm{~Hz}, 3 \mathrm{H}), 0.89(\mathrm{~d}, \mathrm{~J}=6.5 \mathrm{~Hz}, 3 \mathrm{H}), 0.87-0.77(\mathrm{~m}, 2 \mathrm{H}), 0.67$ (d, $\mathrm{J}=7.0 \mathrm{~Hz}, 3 \mathrm{H}), 0.51(\mathrm{~d}, \mathrm{~J}=6.9 \mathrm{~Hz}, 3 \mathrm{H}), 0.37(\mathrm{dd}, \mathrm{J}=15.6,7.0 \mathrm{~Hz}, 1 \mathrm{H}) .{ }^{13} \mathrm{C}$ NMR $(101$ $\left.\mathrm{MHz}, \mathrm{CDCl}_{3}\right) \delta 163.3,151.4,144.8,128.2,125.3,120.7,119.2,116.7,108.6,79.7,72.7$,

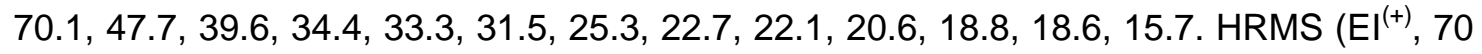
eV) : $\mathrm{C}_{28} \mathrm{H}_{35} \mathrm{O}_{4} \mathrm{~N}_{2} \mathrm{~F}_{4} \mathrm{~S}[\mathrm{M}+1]^{+}$: calcd: 571.2247 , found: 571.2248. $[\alpha]_{D}{ }^{20}=24.8(\mathrm{c}=1.07$, $\left.\mathrm{CH}_{2} \mathrm{Cl}_{2}\right) ; \mathrm{mp}=90^{\circ} \mathrm{C}$.

Synthesis of 3,5-dichloro-N-(2-((S)-4-isopropyl-4,5-dihydrooxazol-2-yl)-6-(((1S,2R,5S) -2-isopropyl-5-methylcyclohexyl)oxy)phenyl)benzenesulfonamide (L10)

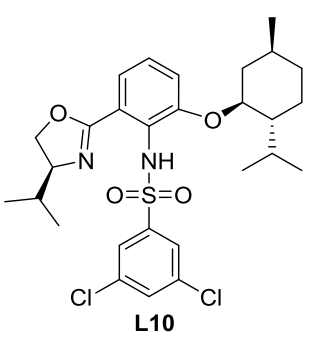


According to the general procedure A, L10 (106.4 mg, $0.19 \mathrm{mmol}$ ) was prepared from L7-4 (89.6 mg, $0.25 \mathrm{mmol}$ ), 3,5-dichlorobenzenesulfonyl chloride $(122.8 \mathrm{mg}, 0.5 \mathrm{mmol}$ ) as white solid in $75 \%$ yield.

${ }^{1} \mathrm{H} \mathrm{NMR}\left(400 \mathrm{MHz}, \mathrm{CDCl}_{3}\right) \delta 7.89(\mathrm{~d}, \mathrm{~J}=1.8 \mathrm{~Hz}, 2 \mathrm{H}), 7.53(\mathrm{t}, \mathrm{J}=1.8 \mathrm{~Hz}, 1 \mathrm{H}), 7.45-7.36$ $(\mathrm{m}, 1 \mathrm{H}), 7.11(\mathrm{t}, \mathrm{J}=8.1 \mathrm{~Hz}, 1 \mathrm{H}), 7.03(\mathrm{~d}, \mathrm{~J}=8.1 \mathrm{~Hz}, 1 \mathrm{H}), 4.42(\mathrm{t}, \mathrm{J}=8.1 \mathrm{~Hz}, 1 \mathrm{H}), 4.14$ (ddd, $\mathrm{J}=17.8,15.1,8.1 \mathrm{~Hz}, 2 \mathrm{H}), 3.93(\mathrm{td}, \mathrm{J}=10.8,4.3 \mathrm{~Hz}, 1 \mathrm{H}), 2.01(\mathrm{~d}, \mathrm{~J}=12.8 \mathrm{~Hz}, 1 \mathrm{H}), 1.87$ (dq, J = 13.2, $6.6 \mathrm{~Hz}, 1 \mathrm{H}), 1.71-1.58(\mathrm{~m}, 2 \mathrm{H}), 1.50(\mathrm{dd}, \mathrm{J}=13.0,3.3 \mathrm{~Hz}, 1 \mathrm{H}), 1.43-1.28$ $(\mathrm{m}, 2 \mathrm{H}), 1.11(\mathrm{~d}, \mathrm{~J}=6.7 \mathrm{~Hz}, 3 \mathrm{H}), 1.00(\mathrm{~d}, \mathrm{~J}=6.7 \mathrm{~Hz}, 3 \mathrm{H}), 0.91(\mathrm{~d}, \mathrm{~J}=6.5 \mathrm{~Hz}, 3 \mathrm{H}), 0.89-$ $0.81(\mathrm{~m}, 2 \mathrm{H}), 0.67(\mathrm{~d}, \mathrm{~J}=7.0 \mathrm{~Hz}, 3 \mathrm{H}), 0.49(\mathrm{~d}, \mathrm{~J}=6.9 \mathrm{~Hz}, 3 \mathrm{H}), 0.34-0.20(\mathrm{~m}, 1 \mathrm{H}) .{ }^{13} \mathrm{C}$ $\operatorname{NMR}\left(101 \mathrm{MHz}, \mathrm{CDCl}_{3}\right) \delta 146.1,135.2,131.5,128.7,125.4,125.0,120.6,116.1,79.0$, 77.3, 72.7, 69.9, 46.8, 39.8, 34.2, 33.2, 31.6, 25.3, 22.7, 22.1, 20.7, 18.7, 15.7. HRMS (El ${ }^{(+)}$, $70 \mathrm{eV}$ ) : $\mathrm{C}_{28} \mathrm{H}_{36} \mathrm{~N}_{2} \mathrm{O}_{4} \mathrm{SCl}_{2}[\mathrm{M}+1]^{+}$: calcd: 566.1763 , found: $566.1773 .[\alpha]_{\mathrm{D}}{ }^{20}=43.7$ (c = 0.64, $\left.\mathrm{CH}_{2} \mathrm{Cl}_{2}\right), \mathrm{mp}=105^{\circ} \mathrm{C}$.

Synthesis of N-(2-((S)-4-isopropyl-4,5-dihydrooxazol-2-yl)-6-(((1S,2R,5S)-2-isopropyl -5-methylcyclohexyl)oxy)phenyl)methanesulfonamide (L11)

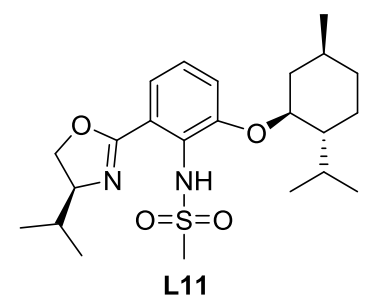

According to the general procedure A, $\mathbf{L} 11(84.0 \mathrm{mg}, 0.19 \mathrm{mmol})$ was prepared from $\mathbf{L} 7-\mathbf{4}$ (89.6 mg, $0.25 \mathrm{mmol}$ ), methanesulfonyl chloride $(57.2 \mathrm{mg}, 0.5 \mathrm{mmol})$ as white solid in $77 \%$ yield.

${ }^{1} \mathrm{H}$ NMR $\left(400 \mathrm{MHz}, \mathrm{CDCl}_{3}\right) \delta 11.82(\mathrm{~s}, 1 \mathrm{H}), 7.37(\mathrm{dd}, \mathrm{J}=7.8,1.2 \mathrm{~Hz}, 1 \mathrm{H}), 7.06(\mathrm{t}, \mathrm{J}=8.0 \mathrm{~Hz}$, $1 \mathrm{H}), 6.98(\mathrm{~d}, \mathrm{~J}=7.7 \mathrm{~Hz}, 1 \mathrm{H}), 4.38(\mathrm{dd}, \mathrm{J}=8.6,7.4 \mathrm{~Hz}, 1 \mathrm{H}), 4.18-4.02(\mathrm{~m}, 2 \mathrm{H}), 3.88(\mathrm{td}, \mathrm{J}$ $=10.7,4.2 \mathrm{~Hz}, 1 \mathrm{H}), 2.38(\mathrm{~s}, 3 \mathrm{H}), 1.96(\mathrm{~d}, \mathrm{~J}=12.1 \mathrm{~Hz}, 1 \mathrm{H}), 1.84(\mathrm{dd}, \mathrm{J}=13.3,6.6 \mathrm{~Hz}, 1 \mathrm{H})$, $1.75-1.65(\mathrm{~m}, 1 \mathrm{H}), 1.60(\mathrm{~d}, \mathrm{~J}=12.6 \mathrm{~Hz}, 1 \mathrm{H}), 1.44(\mathrm{dd}, \mathrm{J}=13.3,3.4 \mathrm{~Hz}, 1 \mathrm{H}), 1.31(\mathrm{dd}, \mathrm{J}=$ 10.7, 7.5 Hz, 2H), $1.10(\mathrm{~d}, \mathrm{~J}=6.7 \mathrm{~Hz}, 3 \mathrm{H}), 0.98(\mathrm{~d}, \mathrm{~J}=6.7 \mathrm{~Hz}, 3 \mathrm{H}), 0.88(\mathrm{~d}, \mathrm{~J}=6.5 \mathrm{~Hz}, 3 \mathrm{H})$, $0.84(\mathrm{~m}, 2 \mathrm{H}), 0.61(\mathrm{~d}, \mathrm{~J}=7.0 \mathrm{~Hz}, 3 \mathrm{H}), 0.48(\mathrm{~d}, \mathrm{~J}=6.9 \mathrm{~Hz}, 3 \mathrm{H}), 0.33(\mathrm{dd}, \mathrm{J}=12.4,10.4 \mathrm{~Hz}$, 1H). ${ }^{13} \mathrm{C} \mathrm{NMR}\left(101 \mathrm{MHz}, \mathrm{CDCl}_{3}\right) \delta 140.5,133.2,132.8,127.9,126.9,120.6,86.4,47.4$, 42.1, 33.5, 31.7, 25.7, 22.9, 21.7, 20.6, 15.1. HRMS (El $\left.{ }^{(+)}, 70 \mathrm{eV}\right): \mathrm{C}_{23} \mathrm{H}_{37} \mathrm{O}_{4} \mathrm{~N}_{2} \mathrm{~S}[\mathrm{M}+1]^{+}$: calcd: 437.2464 , found: $437.2469 .\left[{ }_{0}\right]_{D}^{20}=30.7\left(c=0.44, \mathrm{CH}_{2} \mathrm{Cl}_{2}\right) ; \mathrm{mp}=118^{\circ} \mathrm{C}$.

\section{(3) General procedure B: chromium-catalyzed reaction}

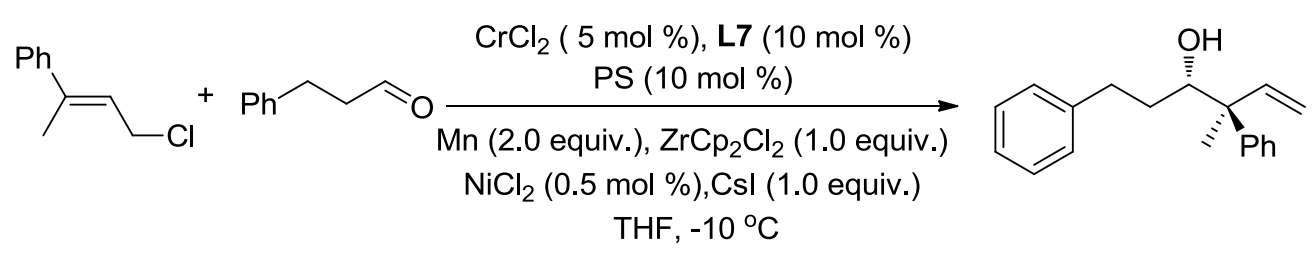

To a mixture of anhydrous chromium(II) chloride (1.2 mg, $0.01 \mathrm{mmol}, 5 \mathrm{mmol} \%$ ), 3,4,5-trifluoro-N-(2-((S)-4-isopropyl-4,5-dihydrooxazol-2-yl)-6-(((1S,2R,5S)-2-isopropyl-5-m ethylcyclohexyl)oxy)phenyl)benzenesulfonamide ( $\mathbf{L 7}, 11.1 \mathrm{mg}, 0.02 \mathrm{mmol}, 10 \mathrm{mmol} \%$ ) 
and Proton sponge (4.4 mg, $0.02 \mathrm{mmol}, 10 \mathrm{mmol} \%)$ was added THF (0.6 mL) under an nitrogen atmosphere. The mixture was stirred vigorously at room temperature for 2 hours before it was transferred into a vessel charged with $\mathrm{Zr}(\mathrm{Cp})_{2} \mathrm{Cl}_{2}(60.0 \mathrm{mg}, 0.2 \mathrm{mmol}, 1.0$ equiv.), Csl (53.2 mg, $0.2 \mathrm{mmol}, 1.0$ equiv.), $\mathrm{NiCl}_{2}(0.1 \mathrm{mg}, 0.001 \mathrm{mmol}, 0.5 \mathrm{mmol} \%$ ) and Manganese powder (22.0 mg, $0.4 \mathrm{mmol}, 2.0$ equiv.). Then allyl chloride (0.4 mmol, 2.0 equiv.) and aldehyde $(0.2 \mathrm{mmol}, 1.0$ equiv.) were added in succession. The resulting suspension was stirred at $-10{ }^{\circ} \mathrm{C}$ for $10 \mathrm{~h}$. After the full consumption of aldehyde, the reaction mixture was diluted with undried $E A$ and the resulting suspension was filtered over a pad of silica gel using EA as eluent. Volatiles were evaporated in vacuo. The residue was purified by chromatography (Petroleum ether : Ethyl acetate $=10: 1$ ) afforded the product.

\section{(4) Evaluation of Chiral Ligands and Other Reaction Parameters}

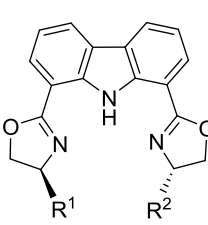

L1: $\quad \mathrm{R}^{1}=\mathrm{R}^{2}=i \mathrm{Pr}$ L1-2: $R^{1}=R^{2}=t B u$ L1-3: $\mathrm{R}^{1}=\mathrm{R}^{2}=\mathrm{PhCH}_{2}$ L1-4: $R^{1}=R^{2}=P h$ L1-5: $\mathrm{R}^{1}=\mathrm{R}^{2}=\mathrm{Et}$
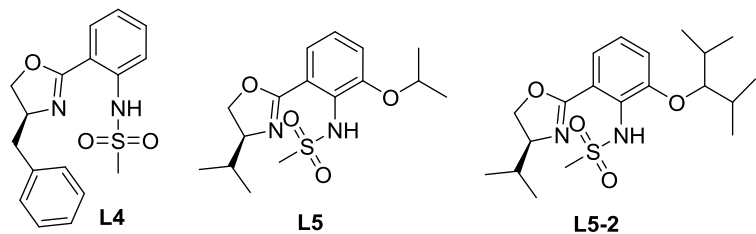<smiles>CC(C)[C@@H]1CC[C@@H](C)C[C@H]1Oc1cccc(C2=N[C@H](C(C)C)CO2)c1NS(=O)(=O)c1ccccc1</smiles>

L6<smiles>CC(C)[C@H]1COC(c2cccc(O[C@@H]3C[C@H](C)CC[C@H]3C(C)C)c2NS(=O)(=O)c2c(F)c(F)cc(F)c2F)=N1</smiles>

L9<smiles>[Z7]c1c(F)cc(S(=O)(=O)Nc2c(O[C@@H]3C[C@H](C)CC[C@H]3C(C)C)cccc2C2=N[C@@H](C(C)C)CO2)cc1F</smiles><smiles>CC(C)[C@@H]1CC[C@@H](C)C[C@H]1Oc1cccc(C2=N[C@H](C(C)C)CO2)c1NS(=O)(=O)c1cc(Cl)cc(Cl)c1</smiles>

L10

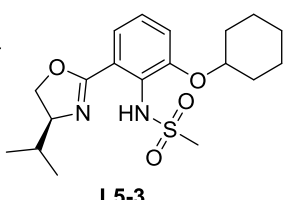

L5-3

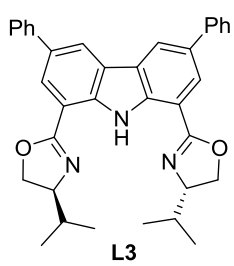<smiles>CC(C)[C@@H]1CC[C@@H](C)C[C@H]1Oc1cccc(C2=N[C@H](C(C)C)CO2)c1NS(=O)(=O)c1cc(F)cc(F)c1</smiles>

L8

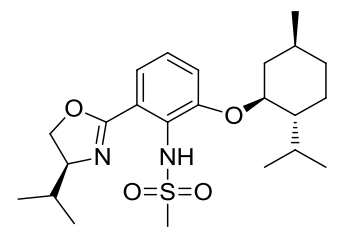

L11

Figure 1. Tested Ligands 


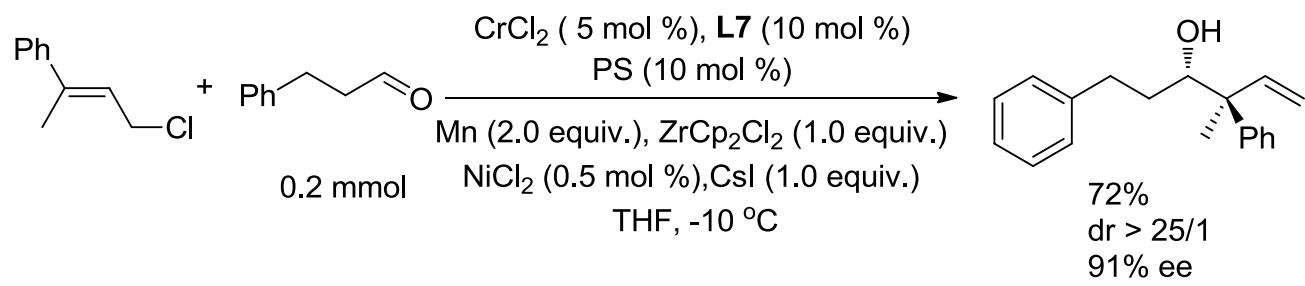

Table 1. Evaluation of Chiral Ligands and Other Reaction Parameters

\begin{tabular}{|c|c|c|c|}
\hline Entry & Deviation from the standard conditions & Yield (\%) & ee $(\%)$ \\
\hline 1 & $\mathbf{L 1 - 2} / \mathrm{NaI} / \mathrm{rt}$ & 50 & 24 \\
\hline 2 & $\mathbf{L 1 - 3} / \mathrm{NaI} / \mathrm{rt}$ & 63 & 45 \\
\hline 3 & L1-4 / NaI / rt & 60 & 45 \\
\hline 4 & L1-5 / NaI / rt & 42 & 33 \\
\hline 5 & $\mathbf{L} \mathbf{2} / \mathrm{NaI} / \mathrm{rt}$ & 70 & 30 \\
\hline 6 & L5-2 / NaI / rt & 69 & 70 \\
\hline 7 & $\mathbf{L 8} / \mathrm{NaI} / \mathrm{rt}$ & 72 & 82 \\
\hline 8 & $\mathbf{L 9} / \mathrm{NaI} / \mathrm{rt}$ & 70 & 71 \\
\hline 9 & $\mathbf{L 1 0} / \mathrm{NaI} / \mathrm{rt}$ & 69 & 72 \\
\hline 10 & $\mathbf{L 5 - 3} / \mathrm{NaI} / \mathrm{rt}$ & 68 & 77 \\
\hline 11 & $\mathbf{L 1}$ & 65 & 50 \\
\hline 12 & $\mathbf{L 2}$ & 65 & 30 \\
\hline 13 & $\mathbf{L 3}$ & 67 & 45 \\
\hline 14 & $\mathbf{L 4}$ & 69 & 7 \\
\hline 15 & $\mathbf{L 5}$ & 66 & 55 \\
\hline 12 & L6 & 70 & 55 \\
\hline 17 & L11 & 50 & 65 \\
\hline 18 & $\mathbf{L} 7 / \mathrm{rt}$ & 70 & 83 \\
\hline 19 & $\mathbf{L} 7$ / CsF / rt & 72 & 83 \\
\hline 20 & $\mathrm{NiCl}_{2}(\mathrm{dme})$ instead of $\mathrm{NiCl}_{2}$ & 70 & 88 \\
\hline 21 & $\begin{array}{l}\mathrm{NiCl}_{2} \text { (2,9-Dimethyl-1,10-phenanthroline) } \\
\text { instead of } \mathrm{NiCl}_{2}\end{array}$ & 50 & 86 \\
\hline 22 & $\mathbf{L 7} / 10{ }^{\circ} \mathrm{C}$ & 71 & 88 \\
\hline 23 & $\mathbf{L 7} / 0{ }^{\circ} \mathrm{C}$ & 72 & 89 \\
\hline 24 & None & 72 & 91 \\
\hline 25 & Without $\mathrm{NiCl}_{2}$ & 50 & 81 \\
\hline 26 & $5 \% \mathrm{CrCl}_{2} / 7 \% \mathbf{L} 7$ & 78 & 85 \\
\hline 27 & Without CsI & 49 & 72 \\
\hline 28 & L7 / DME / $-10{ }^{\circ} \mathrm{C}$ & 72 & 90 \\
\hline 29 & TMSCl instead of $\mathrm{ZrCp}_{2} \mathrm{Cl}_{2}$ & 60 & 83 \\
\hline 30 & $\mathrm{CrCl}_{3} / \mathrm{Mn}$ instead of $\mathrm{CrCl}_{2}$ & 64 & 86 \\
\hline
\end{tabular}


(5) Characterization of products

(3R,4R)-4-methyl-1,4-diphenylhex-5-en-3-ol (2a)<smiles>C=CC(C)(c1ccccc1)C(O)CCc1ccccc1</smiles>

According to the general procedure $B, 2 a(186.5 \mathrm{mg}, 0.70 \mathrm{mmol})$ was prepared from (E)-(4-chlorobut-2-en-2-yl)benzene (333.2 mg, $2 \mathrm{mmol}$ ), 3-phenylpropanal(134.2 mg, 1 $\mathrm{mmol})$ as colorless oil in $70 \%$ yield.

${ }^{1} \mathrm{H}$ NMR $\left(400 \mathrm{MHz}, \mathrm{CDCl}_{3}\right) \delta 7.66-6.91(\mathrm{~m}, 10 \mathrm{H}), 6.27(\mathrm{dd}, J=17.7,10.9 \mathrm{~Hz}, 1 \mathrm{H}), 5.28$ (dd, $J=10.9,1.1 \mathrm{~Hz}, 1 \mathrm{H}), 5.14(\mathrm{dd}, J=17.7,1.1 \mathrm{~Hz}, 1 \mathrm{H}), 4.03-3.89(\mathrm{~m}, 1 \mathrm{H}), 2.99-2.84$ $(\mathrm{m}, 1 \mathrm{H}), 2.69-2.43(\mathrm{~m}, 1 \mathrm{H}), 1.62(\mathrm{dd}, J=7.8,4.2 \mathrm{~Hz}, 2 \mathrm{H}), 1.37(\mathrm{~s}, 3 \mathrm{H}) .{ }^{13} \mathrm{C}$ NMR $(101$ $\left.\mathrm{MHz}, \mathrm{CDCl}_{3}\right) \delta 145.2,143.1,142.2,128.52,128.50,128.3,126.8,126.4,125.8,114.8,76.5$, 49.6, 33.1, 33.0, 19.7. IR (neat) $\mathrm{cm}^{-1} \tilde{\mathrm{v}}: 3577,3467,2956,2856,1494,1260,1057,918$, 698. $\mathrm{HRMS}\left(\mathrm{El}^{(+)}, 70 \mathrm{eV}\right): \mathrm{C}_{19} \mathrm{H}_{22} \mathrm{O}\left[\mathrm{M}^{+}\right.$: calcd: 266.1668 , found: $266.1671 ;[\alpha]_{\mathrm{D}}{ }^{20}=18.4$ (c $=1.04, \mathrm{CH}_{2} \mathrm{Cl}_{2}$ ). $\mathrm{HPLC}$ (Chiralcel IE-H column, hexanes : $i-\mathrm{PrOH}=95: 5,1 \mathrm{~mL} / \mathrm{min}, 210$ $\mathrm{nm}), \mathrm{t}_{\mathrm{minor}}=4.6 \mathrm{~min}, \mathrm{t}_{\mathrm{major}}=5.0 \mathrm{~min}, 91 \%$ ee.

(3S,4S)-3-methyl-3-phenylnon-1-en-4-ol (2b)<smiles>C=C[C@](C)(c1ccccc1)[C@H](O)CCCCCC</smiles>

According to the general procedure $\mathrm{B}, \mathbf{2} \mathbf{b}(34.5 \mathrm{mg}, 0.14 \mathrm{mmol})$ was prepared as colorless oil in $68 \%$ yield.

1H NMR (400 MHz, $\left.\mathrm{CDCl}_{3}\right)$ ठ 7.38-7.31(m, 4H), 7.24- 7.20(m, 1H), 6.29 (dd, J = 17.6, 10.8 $\mathrm{Hz}, 1 \mathrm{H}$ ), 5.29 (dd, J = 10.8, 1.2 Hz, 1H), 5.17 (dd, J = 17.6, $1.2 \mathrm{~Hz}, 1 \mathrm{H}), 3.91(\mathrm{dd}, \mathrm{J}=7.3$, $2.4 \mathrm{~Hz}, 1 \mathrm{H}), 1.38(\mathrm{~s}, 3 \mathrm{H}), 1.35-1.24(\mathrm{~m}, 10 \mathrm{H}), 0.86(\mathrm{t}, \mathrm{J}=6.8 \mathrm{~Hz}, 3 \mathrm{H}) ;{ }^{13} \mathrm{C}$ NMR $(101 \mathrm{MHz}$, $\left.\mathrm{CDCl}_{3}\right) \delta 145.5,143.3,128.4,126.8,126.3,114.6,77.2,49.7,31.8,31.2,29.7,29.3,27.1$, 22.6, 19.8, 14.1. IR (neat) $\mathrm{cm}^{-1} \tilde{\mathrm{v}}$ : 3486, 2926, 1452, 1260, 1030, 800, 732. HRMS (El ${ }^{(+)}, 70$ $\mathrm{eV}): \mathrm{C}_{16} \mathrm{H}_{24}\left[\mathrm{M}-\mathrm{H}_{2} \mathrm{O}\right]^{+}$: calcd: 228.1887, found: 228.1878; $[\mathrm{\alpha}]_{\mathrm{D}}{ }^{20}=-14.0\left(\mathrm{c}=0.60, \mathrm{CH}_{2} \mathrm{Cl}_{2}\right)$; HPLC (Chiralcel IE-H column, hexanes : $i-\mathrm{PrOH}=98: 2,0.8 \mathrm{~mL} / \mathrm{min}, 210 \mathrm{~nm}$ ), $\mathrm{t}_{\text {minor }}=4.5$ $\min , \mathrm{t}_{\text {major }}=5.0 \mathrm{~min}, 91 \%$ ee.

(1R,2R)-1-cyclohexyl-2-methyl-2-phenylbut-3-en-1-ol (2c)<smiles>C=CC(C)(c1ccccc1)C(O)C1CCCCC1</smiles>

According to the general procedure B, 2c $(202.8 \mathrm{mg}, 0.83 \mathrm{mmol})$ was prepared from cyclohexanecarbaldehyde (112.2 mg, $1 \mathrm{mmol}$ ), (E)-(4-chlorobut-2-en-2-yl)benzene (333.2 $\mathrm{mg}, 2 \mathrm{mmol}$ ) as colorless oil in $83 \%$ yield.

${ }^{1} \mathrm{H}$ NMR $\left(400 \mathrm{MHz}, \mathrm{CDCl}_{3}\right) \delta 7.38(\mathrm{dd}, J=8.4,1.1 \mathrm{~Hz}, 2 \mathrm{H}), 7.32(\mathrm{dd}, J=10.4,5.1 \mathrm{~Hz}, 2 \mathrm{H})$, $7.24-7.17(\mathrm{~m}, 1 \mathrm{H}), 6.37$ (dd, $J=17.7,10.9 \mathrm{~Hz}, 1 \mathrm{H}), 5.25(\mathrm{dd}, J=10.9,1.2 \mathrm{~Hz}, 1 \mathrm{H}), 5.13$ (dd, $J=17.7,1.2 \mathrm{~Hz}, 1 \mathrm{H}), 3.74(\mathrm{~s}, 1 \mathrm{H}), 1.88-1.53(\mathrm{~m}, 6 \mathrm{H}), 1.43(\mathrm{~s}, 3 \mathrm{H}), 1.19-1.05(\mathrm{~m}$, 
$5 \mathrm{H}) .{ }^{13} \mathrm{C}$ NMR $\left(101 \mathrm{MHz}, \mathrm{CDCl}_{3}\right) \delta 146.2,144.0,128.3,126.8,126.2,114.0,81.2,50.0,39$. 5, 33.3, 26.8, 26.3, 20.3. IR (neat) $\mathrm{cm}^{-1} \tilde{\mathrm{v}}$ : 3585, 2921, 1445, 1258, 1021, 914, 761, 741. HRMS (El(+), $70 \mathrm{eV}): \mathrm{C}_{17} \mathrm{H}_{22}\left[\mathrm{M}-\mathrm{H}_{2} \mathrm{O}\right]^{+}$: calcd: 226.1718, found: $266.1722 ;[\alpha]_{\mathrm{D}}^{20}=-26.5$ (c $=0.30, \mathrm{CH}_{2} \mathrm{Cl}_{2}$ ). HPLC (Chiralcel IE-H column, hexanes: $i \mathrm{PrOH}=98: 2,0.8 \mathrm{~mL} / \mathrm{min}, 210$ $\mathrm{nm}), \mathrm{t}_{\text {minor }}=6.0 \mathrm{~min}, \mathrm{t}_{\text {major }}=6.9 \mathrm{~min}, 94 \% \mathrm{ee}$.

(3R,4R)-8-chloro-3-methyl-3-phenyloct-1-en-4-ol (2d)<smiles>C=C[C@](C)(c1ccccc1)[C@H](O)CCCCCl</smiles>

According to the general procedure $\mathrm{B}, \mathbf{2} \mathbf{d}(35.4 \mathrm{mg}, 0.14 \mathrm{mmol})$ was prepared as colorless oil in $71 \%$ yield.

${ }^{1} \mathrm{H}$ NMR $\left(400 \mathrm{MHz}, \mathrm{CDCl}_{3}\right)$ ठ 7.38- 7.32(m, 4H), 7.24- 7.20(m, 1H), 6.28 (dd, J = 17.6, 10.8 $\mathrm{Hz}, 1 \mathrm{H}$ ), 5.30 (dd, J = 10.8, 1.2 Hz, 1H), $5.18(\mathrm{dd}, \mathrm{J}=17.6,1.2 \mathrm{~Hz}, 1 \mathrm{H}), 3.91(\mathrm{~d}, \mathrm{~J}=10 \mathrm{~Hz}$, $1 \mathrm{H}), 3.48(\mathrm{t}, \mathrm{J}=6.5 \mathrm{~Hz}, 2 \mathrm{H}), 1.77-1.66(\mathrm{~m}, 4 \mathrm{H}), 1.38(\mathrm{~s}, 3 \mathrm{H}), 1.35-1.30(\mathrm{t}, \mathrm{J}=6.8 \mathrm{~Hz}, 2 \mathrm{H}) ;{ }^{13} \mathrm{C}$ NMR $\left(101 \mathrm{MHz}, \mathrm{CDCl}_{3}\right) \delta$ 143.0, 128.5, 126.7, 126.4, 114.8, 77.1, 49.7, 45.0, 32.5, 30.4, 24.5, 19.7. IR (neat) $\mathrm{cm}^{-1} \tilde{\mathrm{v}}$ : 2961, 2850, 1445, 1260, 1018, 914, 798, 701; HRMS (El ${ }^{(+)}, 70$ eV) : $\mathrm{C}_{18} \mathrm{H}_{20} \mathrm{O}[\mathrm{M}]^{+}$: calcd: 252.1514, found: 252.1516; $[\alpha]_{\mathrm{D}}{ }^{20}=-14.9\left(\mathrm{c}=0.2, \mathrm{CH}_{2} \mathrm{Cl}_{2}\right)$. HPLC (Chiralcel IE-H column, hexanes : $\mathrm{i}-\mathrm{PrOH}=98: 2,1 \mathrm{~mL} / \mathrm{min}, 210 \mathrm{~nm}$ ), $\mathrm{t}_{\text {minor }}=6.3 \mathrm{~min}$, $t_{\text {major }}=7.1 \mathrm{~min}, 91 \%$ ee.

(3R,4R)-3-methyl-3-phenylocta-1,7-dien-4-ol (2e)<smiles>C=CCCC(O)C(C)(C=C)c1ccccc1</smiles>

According to the general procedure $\mathrm{B}, \mathbf{2 e}(35.0 \mathrm{mg}, 0.16 \mathrm{mmol})$ was prepared as colorless oil in $81 \%$ yield.

${ }^{1} \mathrm{H}$ NMR $\left(400 \mathrm{MHz}, \mathrm{CDCl}_{3}\right) \delta 7.41-7.29(\mathrm{~m}, 4 \mathrm{H}), 7.25-7.15(\mathrm{~m}, 1 \mathrm{H}), 6.29(\mathrm{dd}, \mathrm{J}=17.7$, $10.9 \mathrm{~Hz}, 1 \mathrm{H}$ ), $5.87-5.68(\mathrm{~m}, 1 \mathrm{H}), 5.29$ (dd, J = 10.9, $1.2 \mathrm{~Hz}, 1 \mathrm{H}$ ), 5.17 (dd, J = 17.7, $1.2 \mathrm{~Hz}$, $1 \mathrm{H}$ ), 4.98 (ddd, J = 13.6, 11.1, 1.3 Hz, 1H), $3.94(\mathrm{~d}, \mathrm{~J}=9.6 \mathrm{~Hz}, 1 \mathrm{H}), 2.29$ (dt, J = 14.3, 6.5 $\mathrm{Hz}, 1 \mathrm{H}), 2.17-1.94(\mathrm{~m}, 1 \mathrm{H}), 1.76-1.50(\mathrm{~m}, 2 \mathrm{H}), 1.38(\mathrm{~s}, 3 \mathrm{H}) .{ }^{13} \mathrm{C}$ NMR $(101 \mathrm{MHz}, \mathrm{CDCl} 3)$ ठ 145.3, 143.1, 138.6, 128.5, 126.8, 126.8, 126.4, 114.8, 114.7, 76.6, 49.6, 31.1, 30.4, 19.8. IR (neat) $\mathrm{cm}^{-1} \tilde{\mathrm{v}}: 3082,2961,1446,1260,1018,915,764,700 . \operatorname{HRMS}(\operatorname{El}(+), 70 \mathrm{eV})$ : $\mathrm{C}_{15} \mathrm{H}_{20} \mathrm{O}[\mathrm{M}]^{+}$: calcd: 216.1510 , found: $256.1514 ;[\alpha]_{D}{ }^{20}=-14.9\left(\mathrm{c}=0.70, \mathrm{CH}_{2} \mathrm{Cl}_{2}\right)$. HPLC (Chiralcel IE-H column, hexanes $: \mathrm{i}-\mathrm{PrOH}=99: 1,1 \mathrm{~mL} / \mathrm{min}, 210 \mathrm{~nm}$ ), $\mathrm{t}_{\text {minor }}=5.3 \mathrm{~min}$, $\mathrm{t}_{\text {major }}=$ $6.0 \mathrm{~min}, 90 \%$ ee.

(3R,4R, 6S)-3,6,10-trimethyl-3-phenylundeca-1,9-dien-4-ol (2f)<smiles>C=CC(C)(c1ccccc1)C(O)CCCCC=C(C)C</smiles>

According to the general procedure $B, 2 f(47.6 \mathrm{mg}, 0.166 \mathrm{mmol})$ was prepared as yellow oil in $83 \%$ yield. 
${ }^{1} \mathrm{H}$ NMR $\left(400 \mathrm{MHz}, \mathrm{CDCl}_{3}\right) \delta 7.41-7.30(\mathrm{~m}, 4 \mathrm{H}), 7.25-7.18(\mathrm{~m}, 1 \mathrm{H}), 6.30(\mathrm{dd}, J=17.7$, $10.9 \mathrm{~Hz}, 1 \mathrm{H}$ ), 5.29 (dd, $J=10.9,1.2 \mathrm{~Hz}, 1 \mathrm{H}), 5.16(\mathrm{dd}, J=17.7,1.2 \mathrm{~Hz}, 1 \mathrm{H}), 5.07$ (ddd, $J=$ 7.1, 5.8, $1.4 \mathrm{~Hz}, 1 \mathrm{H}$ ), 4.03 (ddd, $J=10.4,3.3,1.5 \mathrm{~Hz}, 1 \mathrm{H}), 2.13-1.82(\mathrm{~m}, 2 \mathrm{H}), 1.66(\mathrm{~d}, J=$ $0.7 \mathrm{~Hz}, 3 \mathrm{H}), 1.58(\mathrm{~d}, J=2.0 \mathrm{~Hz}, 3 \mathrm{H}), 1.51(\mathrm{dd}, J=3.4,1.4 \mathrm{~Hz}, 1 \mathrm{H}), 1.37(\mathrm{~s}, 3 \mathrm{H}), 1.36-$ $1.08(\mathrm{~m}, 2 \mathrm{H}), 1.04-0.94(\mathrm{~m}, 1 \mathrm{H}), 0.85(\mathrm{~d}, J=6.6 \mathrm{~Hz}, 3 \mathrm{H}) \cdot{ }^{13} \mathrm{C} \mathrm{NMR}\left(101 \mathrm{MHz}, \mathrm{CDCl}_{3}\right) \delta$ 145.4, 143.3, 131.1, 128.4, 126.8, 126.3, 124.8, 114.7, 74.8, 49.6, 38.4, 38.2, 29.3, 25.7, 25.6, 19.7, 18.8, 17.7. IR (neat) $\mathrm{cm}^{-1}$ ṽ: 3084, 2962, 1445, 1290, 1020, 917, 739, 700. $\operatorname{HRMS}\left(\mathrm{El}^{(+)}, 70 \mathrm{eV}\right): \mathrm{C}_{20} \mathrm{H}_{30} \mathrm{O}[\mathrm{M}]^{+}:$calcd: 286.2295, found: 286.2297 ; $[\mathrm{\alpha}]_{\mathrm{D}}{ }^{20}=14.0$ (c = $0.60, \mathrm{CH}_{2} \mathrm{Cl}_{2}$ ). HPLC (Chiralcel IE-H column, hexanes : i-PrOH =98:2, $1 \mathrm{~mL} / \mathrm{min}, 210 \mathrm{~nm}$ ), $\mathrm{t}_{\text {minor }}=4.4 \mathrm{~min}, \mathrm{t}_{\text {major }}=4.7 \mathrm{~min}, 92 \%$ ee.

\section{(3R,4R)-4-ethyl-1,4-diphenylhex-5-en-3-ol (2g)}

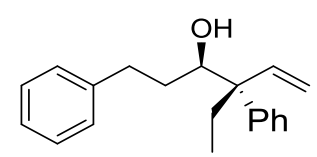

According to the general procedure $\mathrm{B}, \mathbf{2 g}(252.5 \mathrm{mg}, 0.90 \mathrm{mmol})$ was prepared from (E)-(1-chloropent-2-en-3-yl)benzene (361.4 mg, 2 mmol), 3-phenylpropanal(134.2 mg, 1 $\mathrm{mmol})$ as colorless oil in $90 \%$ yield.

${ }^{1} \mathrm{H}$ NMR $\left(400 \mathrm{MHz}, \mathrm{CDCl}_{3}\right) \delta 7.33(\mathrm{~d}, J=4.1 \mathrm{~Hz}, 4 \mathrm{H}), 7.31-7.22(\mathrm{~m}, 3 \mathrm{H}), 7.19(\mathrm{~d}, J=7.2$ $\mathrm{Hz}, 3 \mathrm{H}), 6.13(\mathrm{dd}, J=18.0,11.2 \mathrm{~Hz}, 1 \mathrm{H}), 5.38(\mathrm{~d}, J=11.3 \mathrm{~Hz}, 1 \mathrm{H}), 5.08(\mathrm{~d}, J=18.0 \mathrm{~Hz}$, $1 \mathrm{H}$ ), 4.00 (dd, $J=10.2,4.1 \mathrm{~Hz}, 1 \mathrm{H}$ ), 2.91 (ddd, $J=14.3,9.8,4.8 \mathrm{~Hz}, 1 \mathrm{H}), 2.66(\mathrm{~d}, J=9.1$ $\mathrm{Hz}, 1 \mathrm{H}$ ), 1.81 (dd, $J=14.0,7.0 \mathrm{~Hz}, 3 \mathrm{H}), 1.49$ (ddd, $J=14.5,9.7,5.2 \mathrm{~Hz}, 1 \mathrm{H}$ ), 0.61 (t, $J=$ $7.4 \mathrm{~Hz}, 3 \mathrm{H}) .{ }^{13} \mathrm{C}$ NMR $\left(101 \mathrm{MHz}, \mathrm{CDCl}_{3}\right) \delta 142.4,142.3,140.3,128.6,128.3,128.3,126.4$, 125.8, 116.0, 75.4, 53.7, 33.8, 33.0, 27.8, 8.7. IR (neat) $\mathrm{cm}^{-1} \tilde{\mathrm{v}}: 3479,3025,2964,1454$, 1261, 1031, 918, 757, 698. HRMS $\left(\mathrm{El}^{(+)}, 70 \mathrm{eV}\right): \mathrm{C}_{20} \mathrm{H}_{24} \mathrm{O}[\mathrm{M}]^{+}:$calcd: 280.1830 , found: 280.1827; $[\alpha]_{D}^{20}=20.8\left(\mathrm{c}=0.80, \mathrm{CH}_{2} \mathrm{Cl}_{2}\right.$ ). HPLC (Chiralcel OD-H column, hexanes : $\mathrm{i}-\mathrm{PrOH}=98: 2,0.8 \mathrm{~mL} / \mathrm{min}, 210 \mathrm{~nm}), \mathrm{t}_{\text {minor }}=4.4 \mathrm{~min}, \mathrm{t}_{\text {major }}=4.8 \mathrm{~min}, 92 \%$ ee.

(3R,4R)-3-ethyl-3-phenyldec-1-en-4-ol (2h)

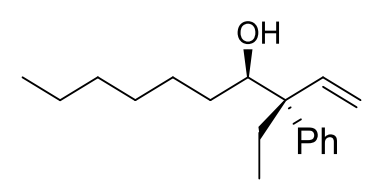

According to the general procedure B, $2 \mathbf{h}(42.2 \mathrm{mg}, 0.16 \mathrm{mmol})$ was prepared as colorless oil in $81 \%$ yield.

${ }^{1} \mathrm{H}$ NMR $\left(400 \mathrm{MHz}, \mathrm{CDCl}_{3}\right) \delta 7.36(\mathrm{~d}, J=4.0 \mathrm{~Hz}, 4 \mathrm{H}), 7.26-7.18(\mathrm{~m}, 1 \mathrm{H}), 6.14(\mathrm{dd}, J=$ 18.0, 11.2 Hz, 1H), $5.42(\mathrm{~d}, J=11.3 \mathrm{~Hz}, 1 \mathrm{H}), 5.16(\mathrm{~d}, J=17.9 \mathrm{~Hz}, 1 \mathrm{H}), 3.98(\mathrm{~d}, J=6.8 \mathrm{~Hz}$, $1 \mathrm{H}), 3.61-3.45(\mathrm{~m}, 2 \mathrm{H}), 1.89-1.75(\mathrm{~m}, 4 \mathrm{H}), 1.56-1.42(\mathrm{~m}, 2 \mathrm{H}), 0.88(\mathrm{dd}, J=10.1,5.1$ $\mathrm{Hz}, 3 \mathrm{H}), 0.67(\mathrm{t}, J=7.4 \mathrm{~Hz}, 3 \mathrm{H}) .{ }^{13} \mathrm{C} \operatorname{NMR}\left(101 \mathrm{MHz}, \mathrm{CDCl}_{3}\right) \delta 142.6,140.6,128.3,128.2$, 126.3, 115.7, 76.2, 53.8, 32.0, 31.9, 29.4, 27.8, 26.9, 22.7, 14.1, 8.8. IR (neat) $\mathrm{cm}^{-1} \tilde{\mathrm{v}}: 3471$, 2926, 1464, 1260, 1062, 915, 760, 701. HRMS (El $\left.{ }^{(+)}, 70 \mathrm{eV}\right): \mathrm{C}_{18} \mathrm{H}_{26}\left[\mathrm{M}-\mathrm{H}_{2} \mathrm{O}\right]^{+}:$calcd: 242.2036, found: $242.2035 ;[\alpha]_{D}^{20}=15.1\left(\mathrm{c}=0.30, \mathrm{CH}_{2} \mathrm{Cl}_{2}\right.$ ). HPLC (Chiralcel OD-H column, hexanes : $i-\mathrm{PrOH}=96: 4,1 \mathrm{~mL} / \mathrm{min}, 210 \mathrm{~nm}), t_{\text {minor }}=4.2 \mathrm{~min}, \mathrm{t}_{\text {major }}=4.0 \mathrm{~min}, 92 \%$ ee. 
<smiles>C=C[C@](CC)(c1ccccc1)C(O)CCCCCl</smiles>

According to the general procedure $B, 2 \mathbf{i}(42.7 \mathrm{mg}, 0.16 \mathrm{mmol})$ was prepared as colorless oil in $80 \%$ yield.

${ }^{1} \mathrm{H}$ NMR $\left(400 \mathrm{MHz}, \mathrm{CDCl}_{3}\right) \delta 7.35-7.30(\mathrm{~m}, 4 \mathrm{H}), 7.26-7.19(\mathrm{~m}, 1 \mathrm{H}), 6.13(\mathrm{dd}, J=18.0$, $11.2 \mathrm{~Hz}, 1 \mathrm{H}), 5.42(\mathrm{~d}, J=11.3 \mathrm{~Hz}, 1 \mathrm{H}), 5.15(\mathrm{~d}, J=17.9 \mathrm{~Hz}, 1 \mathrm{H}), 3.97(\mathrm{~d}, J=6.8 \mathrm{~Hz}, 1 \mathrm{H})$, $3.60-3.40(\mathrm{~m}, 2 \mathrm{H}), 1.91-1.73(\mathrm{~m}, 4 \mathrm{H}), 1.54-1.43(\mathrm{~m}, 2 \mathrm{H}), 0.87$ (dd, J=10.1, $5.1 \mathrm{~Hz}$, 2H), 0.66 (t, $J=7.4 \mathrm{~Hz}, 3 \mathrm{H}) .{ }^{13} \mathrm{C}$ NMR $\left(101 \mathrm{MHz}, \mathrm{CDCl}_{3}\right) \delta 140.3,128.3,128.2,126.4$, 116.1, 76.1, 53.8, 45.1, 32.6, 31.1, 27.7, 24.3, 8.8. IR (neat) $\mathrm{cm}^{-1} \tilde{\mathrm{v}}: 3585,2933,1446,1260$, 1020, 914, 798, 702. HRMS $\left(\mathrm{El}^{(+)}, 70 \mathrm{eV}\right): \mathrm{C}_{16} \mathrm{H}_{23} \mathrm{OCl}[\mathrm{M}]^{+}:$calcd: 266.1432, found: 266.1437; $[\alpha]_{D}{ }^{20}=15.6\left(\mathrm{c}=0.50, \mathrm{CH}_{2} \mathrm{Cl}_{2}\right)$. HPLC (Chiralcel IE-H column, hexanes : $\mathrm{i}-\mathrm{PrOH}$ $=99: 1,1 \mathrm{~mL} / \mathrm{min}, 210 \mathrm{~nm}), \mathrm{t}_{\text {minor }}=8.8 \mathrm{~min}, \mathrm{t}_{\text {major }}=9.6 \mathrm{~min}, 90 \%$ ee.

(3R,4R)-4-cyclohexyl-4-methyl-1-phenylhex-5-en-3-ol (2j)<smiles>C=CC(C)(CCCc1ccccc1)C1CCCCC1</smiles>

According to the general procedure $\mathrm{B}, \mathbf{2} \mathbf{j}(45.2 \mathrm{mg}, 0.17 \mathrm{mmol})$ was prepared as colorless oil in $83 \%$ yield.

${ }^{1} \mathrm{H}$ NMR (400 MHz, CDCl 3 ) ס 7.29 (dd, J = 10.1, 4.6 Hz, 2H), 7.20 (dd, J = 16.3, 7.5 Hz, 3H), 5.79 (dd, J = 17.7, $10.9 \mathrm{~Hz}, 1 \mathrm{H}$ ), $5.22(\mathrm{dd}, \mathrm{J}=11.0,1.5 \mathrm{~Hz}, 1 \mathrm{H}$ ), 5.00 (dd, J = 17.7, $1.6 \mathrm{~Hz}$, $1 \mathrm{H}), 3.59(\mathrm{~d}, \mathrm{~J}=10.3 \mathrm{~Hz}, 1 \mathrm{H}), 3.00-2.88(\mathrm{~m}, 1 \mathrm{H}), 2.62(\mathrm{~m}, 1 \mathrm{H}), 1.80-1.59(\mathrm{~m}, 7 \mathrm{H}), 1.25$ - $1.06(\mathrm{~m}, 4 \mathrm{H}), 0.92-0.85(\mathrm{~m}, 3 \mathrm{H}) .{ }^{13} \mathrm{C} \mathrm{NMR}\left(101 \mathrm{MHz}, \mathrm{CDCl}_{3}\right) \delta$ 142.5, 142.4, 128.5, 128.3, 125.7, 115.5, 73.2, 47.4, 43.1, 33.3, 32.8, 27.7, 27.0, 26.7, 14.5. IR (neat) $\mathrm{cm}^{-1} \tilde{\mathrm{v}}$ : 3481, 2924, 1465, 1261, 1028, 916, 803, 700. HRMS (El $\left.{ }^{(+)}, 70 \mathrm{eV}\right): \mathrm{C}_{19} \mathrm{H}_{28} \mathrm{O}$ : calcd: 272.2139, found: 272.2140; $[\alpha]_{D}{ }^{20}=28.9\left(\mathrm{c}=0.62, \mathrm{CH}_{2} \mathrm{Cl}_{2}\right)$. HPLC (Chiralcel IE-H column, hexanes : $\mathrm{i}-\mathrm{PrOH}=98: 2,1 \mathrm{~mL} / \mathrm{min}, 210 \mathrm{~nm}), \mathrm{t}_{\text {minor }}=6.3 \mathrm{~min}, \mathrm{t}_{\mathrm{majo}} \mathrm{r}=6.8 \mathrm{~min}, 93 \%$ ee.

(3R,4R)-4-(4-chlorophenyl)-4-methyl-1-phenylhex-5-en-3-ol (2k)<smiles>C=CC(c1ccc(Cl)cc1)[C@@H](O)C=C</smiles>

According to the general procedure B, $2 \mathbf{k}(47.5 \mathrm{mg}, 0.16 \mathrm{mmol})$ was prepared as colorless oil in $79 \%$ yield.

${ }^{1} \mathrm{H} \mathrm{NMR}\left(400 \mathrm{MHz}, \mathrm{CDCl}_{3}\right) \delta 7.27(\mathrm{dt}, J=7.5,3.0 \mathrm{~Hz}, 4 \mathrm{H}), 7.24-7.22(\mathrm{~m}, 1 \mathrm{H}), 7.22-7.16$ (m, 2H), $7.13(\mathrm{~d}, J=7.1 \mathrm{~Hz}, 2 \mathrm{H}), 6.21(\mathrm{dd}, J=17.6,10.9 \mathrm{~Hz}, 1 \mathrm{H}), 5.28(\mathrm{~d}, J=10.9 \mathrm{~Hz}, 1 \mathrm{H})$, $5.13(\mathrm{~d}, J=17.6 \mathrm{~Hz}, 1 \mathrm{H}), 3.88(\mathrm{~d}, J=9.1 \mathrm{~Hz}, 1 \mathrm{H}), 2.97-2.77(\mathrm{~m}, 1 \mathrm{H}), 2.59(\mathrm{dt}, J=13.8$, $8.2 \mathrm{~Hz}, 1 \mathrm{H}), 1.61(\mathrm{t}, J=3.3 \mathrm{~Hz}, 2 \mathrm{H}), 1.31(\mathrm{~s}, 3 \mathrm{H}) .{ }^{13} \mathrm{C} \mathrm{NMR}\left(101 \mathrm{MHz}, \mathrm{CDCl}_{3}\right) \delta 143.8$, 
142.8, 141.9, 132.2, 128.5, 128.34, 128.29, 125.8, 115.2, 76.3, 49.3, 33.0, 19.6. IR (neat) $\mathrm{cm}^{-1} \tilde{\mathrm{v}}$ : 3062, 2926, 1454, 1261,1096, 1011, 921, 813, 748, 700. HRMS (El $\left.{ }^{(+)}, 70 \mathrm{eV}\right)$ : $\mathrm{C}_{19} \mathrm{H}_{19} \mathrm{Cl}\left[\mathrm{M}-\mathrm{H}_{2} \mathrm{O}\right]^{+}$: calcd: 282.1182, found: 282.1175; $[\alpha]_{D}{ }^{20}=16.06$ (c $=0.50, \mathrm{CH}_{2} \mathrm{Cl}_{2}$ ). HPLC (Chiralcel IE-H column, hexanes : $i-\mathrm{PrOH}=99: 1,1 \mathrm{~mL} / \mathrm{min}, 210 \mathrm{~nm}$ ), $\mathrm{t}_{\text {minor }}=8.7 \mathrm{~min}$, $t_{\text {major }}=9.8 \mathrm{~min}, 93 \%$ ee.

(1R,2R)-2-(4-chlorophenyl)-1-cyclohexyl-2-methylbut-3-en-1-ol (2I)<smiles>C=CC(c1ccc(Cl)cc1)[C@@H](O)C=C</smiles>

According to the general procedure B, 2l $(209.1 \mathrm{mg}, 0.75 \mathrm{mmol})$ was prepared from(E)-1-chloro-4-(4-chlorobut-2-en-2-yl)benzene(402.2 mg,2 mmol), cyclohexanecarbaldehyde $(112.2 \mathrm{mg}, 1 \mathrm{mmol})$ as colorless oil in $75 \%$ yield.

${ }^{1} \mathrm{H}$ NMR $\left(400 \mathrm{MHz}, \mathrm{CDCl}_{3}\right) \delta 7.61-7.05(\mathrm{~m}, 4 \mathrm{H}), 6.31$ (dd, $\left.J=17.7,10.9 \mathrm{~Hz}, 1 \mathrm{H}\right), 5.25$ (dd, $J=10.8,1.0 \mathrm{~Hz}, 1 \mathrm{H}), 5.12(\mathrm{dd}, J=17.7,1.0 \mathrm{~Hz}, 1 \mathrm{H}), 3.69(\mathrm{~d}, J=3.3 \mathrm{~Hz}, 1 \mathrm{H}), 1.89-1.55$ $(\mathrm{m}, 6 \mathrm{H}), 1.41(\mathrm{~s}, 3 \mathrm{H}), 1.22-0.99(\mathrm{~m}, 5 \mathrm{H}) .{ }^{13} \mathrm{C} \mathrm{NMR}\left(101 \mathrm{MHz}, \mathrm{CDCl}_{3}\right) \delta 144.9,143.7$, 131.9, 128.4, 128.3, 114.3, 81.1, 49.7, 39.6, 33.3, 26.8, 26.3, 20.4. IR (neat) $\mathrm{cm}^{-1} \tilde{\mathrm{v}}: 2961$, 2925, 1493, 1260, 1096, 1014, 799. HRMS (El $\left.{ }^{(+)}, 70 \mathrm{eV}\right): \mathrm{C}_{17} \mathrm{H}_{23} \mathrm{OCl}\left[\mathrm{M}^{+}\right.$: calcd: 278.1441 , found: $278.1437 ;[\alpha]_{D}{ }^{20}=-25.0\left(\mathrm{c}=0.48, \mathrm{CH}_{2} \mathrm{Cl}_{2}\right.$ ). HPLC (Chiralcel IE-H column, hexanes:i-PrOH $=99: 1,1 \mathrm{~mL} / \mathrm{min}, 210 \mathrm{~nm}$ ), $\mathrm{t}_{\text {minor }}=7.3 \mathrm{~min}, \mathrm{t}_{\text {major }}=8.8 \mathrm{~min}, 93 \%$ ee.

(3R,4R)-4-(4-bromophenyl)-4-methyl-1-phenylhex-5-en-3-ol (2m)<smiles>C=C[C@H](O)[C@H](O)CCc1ccccc1</smiles>

According to the general procedure $\mathrm{B}, 2 \mathrm{~m}(40.5 \mathrm{mg}, 0.16 \mathrm{mmol})$ was prepared as colorless oil in $80 \%$ yield.

${ }^{1} \mathrm{H}$ NMR $\left(400 \mathrm{MHz}, \mathrm{CDCl}_{3}\right) \delta 7.45-7.39(\mathrm{~m}, 2 \mathrm{H}), 7.27-7.24(\mathrm{~m}, 2 \mathrm{H}), 7.22-7.10(\mathrm{~m}, 5 \mathrm{H})$, $6.21(\mathrm{dd}, J=17.6,1.2 \mathrm{~Hz}, 1 \mathrm{H}), 5.28(\mathrm{dd}, J=10.9,0.9 \mathrm{~Hz}, 1 \mathrm{H}), 5.13(\mathrm{dd}, J=17.6,0.9 \mathrm{~Hz}$, $1 \mathrm{H}), 3.88(\mathrm{~d}, J=9.1 \mathrm{~Hz}, 1 \mathrm{H}), 2.89(\mathrm{ddd}, J=14.1,8.9,5.5 \mathrm{~Hz}, 1 \mathrm{H}), 2.66-2.54(\mathrm{~m}, 1 \mathrm{H})$, $1.70-1.59(\mathrm{~m}, 2 \mathrm{H}), 1.35(\mathrm{~s}, 3 \mathrm{H}) .{ }^{13} \mathrm{C}$ NMR $\left(101 \mathrm{MHz}, \mathrm{CDCl}_{3}\right) \delta 144.3,142.7,141.9,131.5$, 131.2, 128.7, 128.5, 128.4, 127.2, 125.8, 120.3, 115.2, 76.3, 49.4, 33.0, 19.6, 15.8. IR (neat) $\mathrm{cm}^{-1}$ ṽ: 3576, 2926, 1489, 1261, 1008, 921, 810, 747, 700. HRMS (EI $\left.{ }^{(+)}, 70 \mathrm{eV}\right)$ : $\mathrm{C}_{19} \mathrm{H}_{19} \mathrm{Br}\left[\mathrm{M}-\mathrm{H}_{2} \mathrm{O}\right]^{+}$: calcd: 326.0666, found: 326.0670; $[\alpha]_{D}{ }^{20}=19.6\left(\mathrm{c}=0.53, \mathrm{CH}_{2} \mathrm{Cl}_{2}\right)$. HPLC (Chiralcel IE-H column, hexanes : $\mathrm{i}-\mathrm{PrOH}=99: 1,1 \mathrm{~mL} / \mathrm{min}, 210 \mathrm{~nm}$ ), $\mathrm{t}_{\text {minor }}=10.0$ $\min , \mathrm{t}_{\text {major }}=11.1 \mathrm{~min}, 91 \%$ ee. 
<smiles>C=CC(O)(c1ccc(Br)cc1)C1CCCCC1</smiles>

According to the general procedure $\mathrm{B}, \mathbf{2 n}(47.8 \mathrm{mg}, 0.15 \mathrm{mmol})$ was prepared as colorless oil in $74 \%$ yield.

${ }^{1} \mathrm{H}$ NMR $\left(400 \mathrm{MHz}, \mathrm{CDCl}_{3}\right) \delta 7.43(\mathrm{~d}, J=8.7 \mathrm{~Hz}, 2 \mathrm{H}), 7.26(\mathrm{~d}, J=8.8 \mathrm{~Hz}, 2 \mathrm{H}), 6.30(\mathrm{dd}, J=$ 18, 11.2, Hz, 1H), 5.25 (dd, $J=10.8,1.2 \mathrm{~Hz}, 1 \mathrm{H}), 5.12(\mathrm{dd}, J=17.6,1.2 \mathrm{~Hz}, 1 \mathrm{H}), 3.69(\mathrm{~d}, J$ $=3.5 \mathrm{~Hz}, 1 \mathrm{H}), 1.69-1.58(\mathrm{~m}, 6 \mathrm{H}), 1.41(\mathrm{~s}, 3 \mathrm{H}), 1.24-0.95(\mathrm{~m}, 5 \mathrm{H}) .{ }^{13} \mathrm{C} \mathrm{NMR}(101 \mathrm{MHz}$, $\left.\mathrm{CDCl}_{3}\right) \delta 145.4,143.6,131.3,128.7,127.2,114.4,81.0,49.8,39.5,33.3,26.7,26.2,20.3$. IR (neat) $\mathrm{cm}^{-1} \tilde{\mathrm{v}}: 3481,2923,1489,1260,1008,918,800$. HRMS $\left(\mathrm{El}^{(+)}, 70 \mathrm{eV}\right): \mathrm{C}_{17} \mathrm{H}_{21} \mathrm{Br}$ $\left[\mathrm{M}-\mathrm{H}_{2} \mathrm{O}\right]^{+}$: calcd: 304.0832, found: 304.0827; $[\alpha]_{D}{ }^{20}=-29.2\left(\mathrm{c}=0.36, \mathrm{CH}_{2} \mathrm{Cl}_{2}\right)$. HPLC (Chiralcel IE-H column, hexanes : $i-\mathrm{PrOH}=99: 1,1 \mathrm{~mL} / \mathrm{min}, 210 \mathrm{~nm}$ ), $\mathrm{t}_{\mathrm{minor}}=8.4 \mathrm{~min}, \mathrm{t}_{\mathrm{major}}=$ $10.4 \min , 91 \%$ ee.

(3R,4R)-4-(4-fluorophenyl)-4-methyl-1-phenylhex-5-en-3-ol (20)<smiles>C=CC(c1ccc(F)cc1)[C@@H](O)C=C</smiles>

According to the general procedure $\mathrm{B}, 20(43.2 \mathrm{mg}, 0.15 \mathrm{mmol})$ was prepared as colorless oil in $76 \%$ yield.

${ }^{1} \mathrm{H}$ NMR (400 MHz, CDCl $)$ ) $7.34-7.22(\mathrm{~m}, 4 \mathrm{H}), 7.18(\mathrm{t}, J=7.3 \mathrm{~Hz}, 1 \mathrm{H}), 7.13(\mathrm{~d}, J=6.9$ $\mathrm{Hz}, 2 \mathrm{H}$ ), 6.99 (t, $J=8.8 \mathrm{~Hz}, 2 \mathrm{H}), 6.23(\mathrm{dd}, J=17.6,10.9 \mathrm{~Hz}, 1 \mathrm{H}), 5.28(\mathrm{dd}, J=10.9,1.0 \mathrm{~Hz}$, $1 \mathrm{H}), 5.13(\mathrm{dd}, J=17.7,1.0 \mathrm{~Hz}, 1 \mathrm{H}), 3.88(\mathrm{~d}, J=9.2 \mathrm{~Hz}, 1 \mathrm{H}), 2.89-2.82(\mathrm{~m}, 1 \mathrm{H}), 2.74-2.34$ $(\mathrm{m}, 1 \mathrm{H}), 1.61(\mathrm{t}, J=3.3 \mathrm{~Hz}, 2 \mathrm{H}), 1.36(\mathrm{~s}, 3 \mathrm{H}) .{ }^{13} \mathrm{C} \mathrm{NMR}\left(101 \mathrm{MHz}, \mathrm{CDCl}_{3}\right) \delta$ 143.0, 142.0, 128.5, 128.4, 128.4, 128.3, 125.8, 115.3, 115.04, 114.95, 76.4, 49.2, 33.04, 32.98, 9.8. IR (neat) $\mathrm{cm}^{-1} \tilde{\text { v: }}$ 3470, 2925, 1495, 1260, 1008, 920, 819, 747, 700. HRMS (El $\left.{ }^{(+)}, 70 \mathrm{eV}\right)$ : $\mathrm{C}_{19} \mathrm{H}_{19} \mathrm{~F}\left[\mathrm{M}-\mathrm{H}_{2} \mathrm{O}\right]^{+}$: calcd: 266.1471, found: 266.1465. [ $\left.\alpha\right]_{\mathrm{D}}{ }^{20}=15.6$ (c $=0.48, \mathrm{CH}_{2} \mathrm{Cl}_{2}$ ). HPLC (Chiralcel IE-H column, hexanes:i-PrOH = 99:1, $1 \mathrm{~mL} / \mathrm{min}, 210 \mathrm{~nm}$ ), $\mathrm{t}_{\text {minor }}=6.9 \mathrm{~min}$, $t_{\text {major }}=8.3 \mathrm{~min}, 94 \%$ ee.

(3R,4R)-4-(4-fluorophenyl)-4-methyl-1-phenylhex-5-en-3-ol (2p)<smiles>C=CC(O)C(C=C)(c1ccc(F)cc1)C1CCCCC1</smiles>

According to the general procedure $\mathrm{B}, \mathbf{2 p}(37.8 \mathrm{mg}, 0.14 \mathrm{mmol})$ was prepared as colorless oil in $72 \%$ yield. 
${ }^{1} \mathrm{H}$ NMR $\left(400 \mathrm{MHz}, \mathrm{CDCl}_{3}\right) \delta 7.56-7.29(\mathrm{~m}, 2 \mathrm{H}), 7.00(\mathrm{t}, J=8.7 \mathrm{~Hz}, 2 \mathrm{H}), 6.33(\mathrm{dd}, J=$ $17.7,10.9 \mathrm{~Hz}, 1 \mathrm{H}), 5.25(\mathrm{dd}, J=10.9,1.0 \mathrm{~Hz}, 1 \mathrm{H}), 5.12(\mathrm{dd}, J=17.7,1.1 \mathrm{~Hz}, 1 \mathrm{H}), 3.69(\mathrm{~d}$, $J=3.3 \mathrm{~Hz}, 1 \mathrm{H}), 1.85-1.46(\mathrm{~m}, 6 \mathrm{H}), 1.45(\mathrm{~s}, 3 \mathrm{H}), 1.19-0.99(\mathrm{~m}, 5 \mathrm{H}) .{ }^{13} \mathrm{C}$ NMR $(101 \mathrm{MHz}$, $\left.\mathrm{CDCl}_{3}\right) \delta 143.9,141.8,128.5,128.4,115.1,114.9,114.2,81.2,49.6,39.5,33.3,26.8,26.3$, 20.6. ${ }^{19} \mathrm{~F} \mathrm{NMR}\left(376 \mathrm{MHz}, \mathrm{CDCl}_{3}\right.$ ) $\delta-117.15,-117.16$. IR (neat) $\mathrm{cm}^{-1} \mathrm{v}: 2959,2927,1454$, 1260, 1015, 921, 800, 700. HRMS $\left(\mathrm{EI}^{(+)}, 70 \mathrm{eV}\right): \mathrm{C}_{19} \mathrm{H}_{21} \mathrm{~F}\left[\mathrm{M}-\mathrm{H}_{2} \mathrm{O}\right]^{+}$: calcd: 244.1633 , found: 244.1627; $[\alpha]_{D}{ }^{20}=-15.5\left(\mathrm{c}=0.30, \mathrm{CH}_{2} \mathrm{Cl}_{2}\right)$. HPLC (Chiralcel IE-H column, hexanes : $i-\mathrm{PrOH}=99: 1,1 \mathrm{~mL} / \mathrm{min}, 210 \mathrm{~nm}), \mathrm{t}_{\text {minor }}=5.6 \mathrm{~min}, \mathrm{t}_{\text {major }}=6.7 \mathrm{~min}, 95 \%$ ee.

(3R,4S)-4,8-dimethyl-1-phenyl-4-vinyInon-7-en-3-ol (2q)

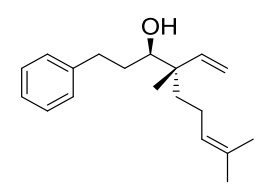

According to the general procedure $B, 2 q(242.4 \mathrm{mg}, 0.89 \mathrm{mmol})$ was prepared from (E)-1-bromo-3,7-dimethylocta-2,6-diene (2.0 mmol, $434.4 \mathrm{mg}$ ), 3-phenylpropanal(134.2 mg, $1 \mathrm{mmol}$ ) as colorless oil in $89 \%$ yield.

${ }^{1} \mathrm{H}$ NMR $\left(400 \mathrm{MHz}, \mathrm{CDCl}_{3}\right) \delta 7.33-7.23(\mathrm{~m}, 2 \mathrm{H}), 7.23-7.12(\mathrm{~m}, 3 \mathrm{H}), 5.72(\mathrm{dd}, J=17.6$, $10.9 \mathrm{~Hz}, 1 \mathrm{H}), 5.20(\mathrm{dd}, J=10.8,1.3 \mathrm{~Hz}, 1 \mathrm{H}), 5.11-4.98(\mathrm{~m}, 2 \mathrm{H}), 3.29(\mathrm{~d}, J=10.4 \mathrm{~Hz}, 1 \mathrm{H})$, $2.99-2.82(\mathrm{~m}, 1 \mathrm{H}), 2.59(\mathrm{ddd}, J=13.7,9.9,6.9 \mathrm{~Hz}, 1 \mathrm{H}), 1.92-1.72(\mathrm{~m}, 3 \mathrm{H}), 1.65(\mathrm{~s}, 3 \mathrm{H})$, $1.55(\mathrm{~s}, 3 \mathrm{H}), 1.42-1.19(\mathrm{~m}, 3 \mathrm{H}), 0.95(\mathrm{~s}, 3 \mathrm{H}) .{ }^{13} \mathrm{C} \mathrm{NMR}\left(101 \mathrm{MHz}, \mathrm{CDCl}_{3}\right) \delta 144.1,142.4$, 128.5, 128.3, 125.7, 124.6, 115.4, 107.2, 76.3, 45.1, 37.4, 33.3, 32.8, 25.7, 22.7, 17.6, 16.4.

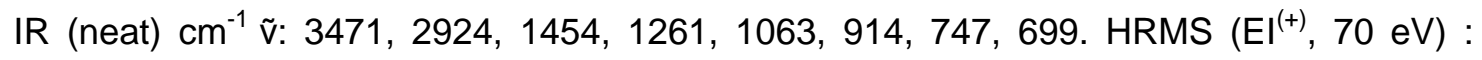
$\mathrm{C}_{19} \mathrm{H}_{28} \mathrm{O}[\mathrm{M}]^{+}$: calcd: 272.2145 , found: $272.2140 .[\alpha]_{\mathrm{D}}{ }^{20}=17.4\left(\mathrm{c}=0.22, \mathrm{CH}_{2} \mathrm{Cl}_{2}\right)$. HPLC (Chiralcel IE-H column, hexanes: $\mathrm{i}-\mathrm{PrOH}=98: 2,1 \mathrm{~mL} / \mathrm{min}, 210 \mathrm{~nm}$ ), $\mathrm{t}_{\text {minor }}=11.0 \mathrm{~min}, \mathrm{t}_{\text {major }}=$ $11.8 \mathrm{~min}, 95 \%$ ee.

(1S,2S)-2,6-dimethyl-1-phenyl-2-vinylhept-5-en-1-ol (2r)<smiles>C=CC(O)c1ccccc1CC=CC(C)C</smiles>

According to the general procedure $B, 2 r(d r=4: 1)(40.1 \mathrm{mg}, 0.16 \mathrm{mmol})$ was prepared as colorless oil in $82 \%$ yield $^{[3]}$.

Major isomer: ${ }^{1} \mathrm{H}$ NMR $\left(400 \mathrm{MHz}, \mathrm{CDCl}_{3}\right) \delta 7.41-7.17(\mathrm{~m}, 5 \mathrm{H}), 5.86(\mathrm{dd}, \mathrm{J}=17.6,10.8 \mathrm{~Hz}$, $1 \mathrm{H}), 5.29(\mathrm{~d}, \mathrm{~J}=10.8 \mathrm{~Hz}, 1 \mathrm{H}), 5.10(\mathrm{~d}, \mathrm{~J}=17.6 \mathrm{~Hz}, 1 \mathrm{H}), 5.05(\mathrm{~d}, \mathrm{~J}=7.4 \mathrm{~Hz}, 1 \mathrm{H}), 4.43(\mathrm{~s}$, $1 \mathrm{H}), 1.85$ (dd, J = 16.1, 7.9 Hz, 2H), $1.65(\mathrm{~s}, 3 \mathrm{H}), 1.55(\mathrm{~s}, 3 \mathrm{H}), 1.32-1.19(\mathrm{~m}, 2 \mathrm{H}), 0.93(\mathrm{~s}$, $3 \mathrm{H}) \cdot\left[\mathrm{\alpha}_{\mathrm{D}}{ }^{20}=-7.1\left(\mathrm{c}=0.49, \mathrm{CH}_{2} \mathrm{Cl}_{2}\right)\right.$. HPLC (Chiralcel OD-H column, hexanes : $i-\mathrm{PrOH}=$ $97: 3,1 \mathrm{~mL} / \mathrm{min}, 210 \mathrm{~nm}), \mathrm{t}_{\text {major }}=6.7 \mathrm{~min}, \mathrm{t}_{\text {minor }}=9.0 \mathrm{~min}, 93 \%$ ee.

(3R,4S,E)-4,8-dimethyl-1-phenyl-4-vinylnona-1,7-dien-3-ol (2s) 


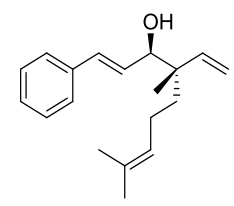

According to the general procedure $B, 2 \mathbf{s}(\mathrm{dr}=5: 1)(44.3 \mathrm{mg}, 0.16 \mathrm{mmol})$ was prepared as colorless oil in $82 \%$ yield $^{[3]}$.

Major isomer: ${ }^{1} \mathrm{H}$ NMR (400 MHz, $\left.\mathrm{CDCl}_{3}\right) \delta 7.47-7.36(\mathrm{~m}, 2 \mathrm{H}), 7.32(\mathrm{t}, \mathrm{J}=7.5 \mathrm{~Hz}, 2 \mathrm{H})$, $7.24(\mathrm{t}, \mathrm{J}=4.7 \mathrm{~Hz}, 1 \mathrm{H}), 6.59(\mathrm{dd}, \mathrm{J}=15.8,5.9 \mathrm{~Hz}, 1 \mathrm{H}), 6.25(\mathrm{dd}, \mathrm{J}=7.0,2.9 \mathrm{~Hz}, 1 \mathrm{H}), 6.20$ $(d, J=7.4 \mathrm{~Hz}, 1 \mathrm{H}$ ), $5.86(\mathrm{dd}, \mathrm{J}=17.6,10.8 \mathrm{~Hz}, 1 \mathrm{H}$ ), 5.28 (dd, J = 10.8, 1.3 Hz, $1 \mathrm{H}$ ), 5.13 (dd, J = 17.6, 1.3 Hz, 1H), $3.99(\mathrm{~d}, \mathrm{~J}=7.4 \mathrm{~Hz}, 1 \mathrm{H}), 1.93(\mathrm{dd}, \mathrm{J}=16.6,7.6 \mathrm{~Hz}, 2 \mathrm{H}), 1.67$ $(\mathrm{s}, 3 \mathrm{H}), 1.58(\mathrm{~s}, 3 \mathrm{H}), 1.47-1.35(\mathrm{~m}, 2 \mathrm{H}), 1.02(\mathrm{~d}, \mathrm{~J}=7.8 \mathrm{~Hz}, 3 \mathrm{H}) \cdot[\mathrm{\alpha}]_{\mathrm{D}}^{20}=22.12(\mathrm{c}=0.97$, $\mathrm{CH}_{2} \mathrm{Cl}_{2}$ ). HPLC (Chiralcel OD-H column, hexanes : $i-\mathrm{PrOH}=97: 3,1 \mathrm{~mL} / \mathrm{min}, 210 \mathrm{~nm}$ ), $\mathrm{t}_{\text {minor }}$ $=10.4 \mathrm{~min}, \mathrm{t}_{\mathrm{major}}=15.4 \mathrm{~min}, 90 \%$ ee.

(3R,4S)-4-(((4-chlorophenyl)sulfonyl)methyl)-4-methyl-1-phenylhex-5-en-3-ol (2t)<smiles>C=CC(O)(CCc1ccccc1)C(=O)Oc1ccc(Cl)cc1</smiles>

According to the general procedure $\mathrm{B}, \mathbf{2 t}(61,4 \mathrm{mg}, 0.16 \mathrm{mmol})$ was prepared as colorless oil in $81 \%$ yield.

${ }^{1} \mathrm{H}$ NMR $\left(400 \mathrm{MHz}, \mathrm{CDCl}_{3}\right) \delta 7.83(\mathrm{~d}, \mathrm{~J}=8.6 \mathrm{~Hz}, 2 \mathrm{H}), 7.53(\mathrm{~d}, \mathrm{~J}=8.5 \mathrm{~Hz}, 2 \mathrm{H}), 7.31-7.23$ $(\mathrm{m}, 2 \mathrm{H}), 7.20(\mathrm{~d}, \mathrm{~J}=7.0 \mathrm{~Hz}, 3 \mathrm{H}), 5.90(\mathrm{dd}, \mathrm{J}=17.6,10.9 \mathrm{~Hz}, 1 \mathrm{H}), 5.14(\mathrm{~d}, \mathrm{~J}=10.9 \mathrm{~Hz}, 1 \mathrm{H})$, $5.03(\mathrm{~d}, \mathrm{~J}=17.5 \mathrm{~Hz}, 1 \mathrm{H}), 3.98(\mathrm{~d}, \mathrm{~J}=8.0 \mathrm{~Hz}, 1 \mathrm{H}), 3.92(\mathrm{dd}, \mathrm{J}=9.0,5.8 \mathrm{~Hz}, 1 \mathrm{H}), 3.47(\mathrm{~d}, \mathrm{~J}$ $=14.0 \mathrm{~Hz}, 1 \mathrm{H}), 3.03-2.81(\mathrm{~m}, 3 \mathrm{H}), 2.70-2.53(\mathrm{~m}, 1 \mathrm{H}), 1.34(\mathrm{~s}, 3 \mathrm{H}) .{ }^{13} \mathrm{C} \mathrm{NMR}(101 \mathrm{MHz}$, $\left.\mathrm{CDCl}_{3}\right) \delta 142.1,140.2,132.0,129.7,129.2,128.5,128.4,125.9,123.9,115.4,75.1,63.1$, 46.4, 33.4, 33.1, 19.8. HRMS (El $\left.{ }^{(+)}, 70 \mathrm{eV}\right): \mathrm{C}_{20} \mathrm{H}_{23} \mathrm{O}_{3} \mathrm{SCl}[\mathrm{M}]^{+}$: calcd: 378.1052, found: 378.1056. $[\alpha]_{D}{ }^{20}=-6.3\left(\mathrm{c}=0.57, \mathrm{CH}_{2} \mathrm{Cl}_{2}\right)$. HPLC (Phenomenex Lux cellulose-1 $(250 \times 4.6$ $\mathrm{mm} \times 5 \mathrm{um}$ ) column, $\left.\mathrm{CH}_{3} \mathrm{OH}: \mathrm{H}_{2} \mathrm{O}=90: 10,0.7 \mathrm{~mL} / \mathrm{min}, 254 \mathrm{~nm}\right), \mathrm{t}_{\text {minor }}=12.2 \mathrm{~min}, \mathrm{t}_{\text {major }}=$ $13.5 \mathrm{~min}, 92 \%$ ee.

(2R,3S)-1-(4-methoxyphenyl)-3,7-dimethyl-3-vinyloct-6-en-2-ol (2u)<smiles>C=C[C@H](O)[C@@H](/C=C/C=C(C)C)Cc1ccc(OC)cc1</smiles>

According to the general procedure $\mathrm{B}, \mathbf{2 u}(102.4 \mathrm{mg}, 0.36 \mathrm{mmol})$ was prepared as colorless oil in $71 \%$ yield from 2-(4-methoxyphenyl)acetaldehyde $(0.5 \mathrm{mmol}, 75.1 \mathrm{mg})$, (E)-1-bromo-3,7-dimethylocta-2,6-diene $(1.0 \mathrm{mmol}, 217.2 \mathrm{mg})^{[4]}$.

${ }^{1} \mathrm{H}$ NMR $\left(400 \mathrm{MHz}, \mathrm{CDCl}_{3}\right) \delta 7.15(\mathrm{~d}, \mathrm{~J}=8.3 \mathrm{~Hz}, 2 \mathrm{H}), 6.85(\mathrm{~d}, \mathrm{~J}=8.2 \mathrm{~Hz}, 2 \mathrm{H}), 5.85(\mathrm{dd}, \mathrm{J}=$ 17.6, $10.9 \mathrm{~Hz}, 1 \mathrm{H}), 5.22(\mathrm{~d}, \mathrm{~J}=10.7 \mathrm{~Hz}, 1 \mathrm{H}), 5.11-5.07(\mathrm{~m}, 2 \mathrm{H}), 3.79(\mathrm{~s}, 3 \mathrm{H}), 3.49(\mathrm{~d}, \mathrm{~J}=$ 
$10.7 \mathrm{~Hz}, 1 \mathrm{H}), 2.83(\mathrm{~d}, \mathrm{~J}=13.7 \mathrm{~Hz}, 1 \mathrm{H}), 2.56-2.27(\mathrm{~m}, 1 \mathrm{H}), 2.06-1.82(\mathrm{~m}, 2 \mathrm{H}), 1.68(\mathrm{~s}$, $3 \mathrm{H}), 1.60(\mathrm{~s}, 3 \mathrm{H}), 1.54-1.41(\mathrm{~m}, 2 \mathrm{H}), 1.08(\mathrm{~s}, 3 \mathrm{H}) .[\alpha]_{\mathrm{D}}^{20}=22.7,\left(\mathrm{c}=0.73, \mathrm{CH}_{2} \mathrm{Cl}_{2}\right) . \mathrm{HPLC}$ (Chiralcel IE-H column, hexanes : $i-\mathrm{PrOH}=99: 1,0.6 \mathrm{~mL} / \mathrm{min}, 210 \mathrm{~nm}$ ), $t_{\text {minor }}=14.8 \mathrm{~min}$, $t_{\text {major }}=18.6 \mathrm{~min}, 91 \%$ ee.

(1R,2R)-1-cyclohexyl-2-methyl-2-phenylbutane-1,4-diol

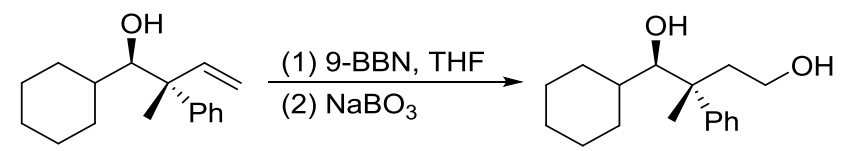

To a $50 \mathrm{~mL}$, 3-necked, round-bottomed flask equipped with a reflux condenser containing a solution of $3 \mathrm{~g}$ ( $48.8 \mathrm{mg}, 0.2 \mathrm{mmol}, 1.0$ equiv.) in $2 \mathrm{~mL}$ of THF was added a solution of 9-BBN in THF solution ( $3 \mathrm{~mL}, 0.5 \mathrm{M}, 0.6 \mathrm{mmol}, 3.0$ equiv.). The solution was then heated to reflux for $3 \mathrm{~h}$. The reaction mixture was cooled at $0{ }^{\circ} \mathrm{C}$ and $\mathrm{H}_{2} \mathrm{O}(0.7 \mathrm{~mL})$, sodium perborate (277.7 mg, $1.8 \mathrm{mmol}, 9.0$ equiv.) and sodium hydroxide $(66.7 \mathrm{mg}, 1.8 \mathrm{mmol}, 9.0$ equiv.) were added, after which the mixture was stirred at $50^{\circ} \mathrm{C}$ in an oil bath for $4 \mathrm{~h}$. The phases were then separated and the aqueous layer was extracted with ethyl acetate $(3 \times 50 \mathrm{~mL})$. The organic extracts were combined, dried $\left(\mathrm{MgSO}_{4}\right)$, and concentrated. The residue was purified by column chromatography (silicagel, Petroleum ether : Ethyl acetate,3:1 to $1: 1$ ) to (1S,2S)-1-cyclohexyl-2-methyl-2-phenylbutane-1,4-diol as a white powder ${ }^{[3 \mathrm{a}]}$ in $90 \%$ yield. ${ }^{1} \mathrm{H} \mathrm{NMR}\left(400 \mathrm{MHz}, \mathrm{CDCl}_{3}\right)$ ठ $7.38-7.28(\mathrm{~m}, 4 \mathrm{H}), 7.21$ (dd, J = 8.7, $\left.4.4 \mathrm{~Hz}, 1 \mathrm{H}\right), 3.70$ (d, J $=3.5 \mathrm{~Hz}, 1 \mathrm{H}), 3.59(\mathrm{dt}, \mathrm{J}=11.0,6.6 \mathrm{~Hz}, 1 \mathrm{H}), 3.48(\mathrm{dt}, \mathrm{J}=10.8,6.6 \mathrm{~Hz}, 1 \mathrm{H}), 2.09(\mathrm{dt}, \mathrm{J}=$ 13.4, $6.6 \mathrm{~Hz}, 1 \mathrm{H}), 2.03-1.92(\mathrm{br}, 2 \mathrm{H}), 1.65(\mathrm{br}, 2 \mathrm{H}), 1.53(\mathrm{~s}, 3 \mathrm{H}), 1.38(\mathrm{br}, 1 \mathrm{H}), 1.27$ $(\mathrm{br}, 1 \mathrm{H}), 1.14-0.89(\mathrm{~m}, 5 \mathrm{H}) .[\mathrm{\alpha}]_{\mathrm{D}}^{20}=13.8,\left(\mathrm{c}=0.3, \mathrm{CH}_{2} \mathrm{Cl}_{2}\right)$.

\section{(S,E)-4-(3,7-dimethyl-3-vinylocta-1,6-dien-1-yl)phenol ((S)-Bakuchiol)}

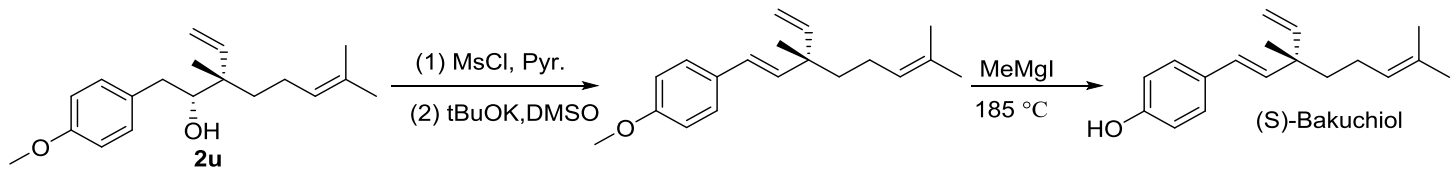

\section{(S,E)-1-(3,7-dimethyl-3-vinylocta-1,6-dien-1-yl)-4-methoxybenzene}

To a solution of $2 \mathbf{u}(0.145 \mathrm{~g}, 0.5 \mathrm{mmol})$ in $3 \mathrm{ml}$ of dry pyridine was added $\mathrm{MeSO}_{2} \mathrm{Cl}(63 \mathrm{mg}$, $0.55 \mathrm{mmol})$, and the mixture was stirred at r.t. for $10 \mathrm{~h} . \mathrm{H}_{2} \mathrm{O}(0.5 \mathrm{ml})$ was added, and the product was extracted with $\mathrm{Et}_{2} \mathrm{O}$. The extracts were washed with $\mathrm{H}_{2} \mathrm{O}$ and dried $\left(\mathrm{Na}_{2} \mathrm{SO}_{4}\right)$. The solvent was removed under reduced pressure to give yellow oil without further purification. To a solution of the above yellow oil $(74 \mathrm{mg}, 0.2 \mathrm{mmol}$ ) in $0.5 \mathrm{ml}$ of dry DMSO was added $t \mathrm{BuOK}(44.8 \mathrm{mg}, 0.4 \mathrm{mmol})$ in one portion, and the mixture was stirred at r.t. for $5 \mathrm{~h}$. The mixture was poured into $3 \mathrm{ml}$ of $\mathrm{H}_{2} \mathrm{O}$, and the product was extracted with $\mathrm{Et}_{2} \mathrm{O}$. The combined organic phase was washed with aq. $\mathrm{NaCl}$ and dried $\left(\mathrm{Na}_{2} \mathrm{SO}_{4}\right)$. The solvent was removed under reduced pressure, and the crude product was purified by column chromatography (silicagel; Petroleum ether) to afford (S,E)-1-(3,7-dimethyl-3-vinylocta-1,6 -dien-1-yl)-4-methoxybenzene (49 mg, 91.0\% for two steps), Colorless oil ${ }^{[4]}$. ${ }^{1} \mathrm{H}$ NMR (400 $\left.\mathrm{MHz}, \mathrm{CDCl}_{3}\right) \delta 7.30(\mathrm{~d}, \mathrm{~J}=8.5 \mathrm{~Hz}, 2 \mathrm{H}), 6.84(\mathrm{~d}, \mathrm{~J}=8.6 \mathrm{~Hz}, 2 \mathrm{H}), 6.26(\mathrm{~d}, \mathrm{~J}=16.0 \mathrm{~Hz}, 1 \mathrm{H})$, $6.06(\mathrm{~d}, \mathrm{~J}=16.2 \mathrm{~Hz}, 1 \mathrm{H}), 5.88(\mathrm{dd}, \mathrm{J}=17.3,10.8 \mathrm{~Hz}, 1 \mathrm{H}), 5.11(\mathrm{t}, \mathrm{J}=7.2 \mathrm{~Hz}, 1 \mathrm{H}), 5.02$ (dd, $\mathrm{J}=14.0,8.3 \mathrm{~Hz}, 2 \mathrm{H}), 3.80(\mathrm{~s}, 3 \mathrm{H}), 1.95(\mathrm{dd}, \mathrm{J}=16.7,8.0 \mathrm{~Hz}, 2 \mathrm{H}), 1.67(\mathrm{~s}, 3 \mathrm{H}), 1.59(\mathrm{~s}, 3 \mathrm{H})$, 
$1.48(\mathrm{dd}, \mathrm{J}=20.5,12.1 \mathrm{~Hz}, 2 \mathrm{H}), 1.20(\mathrm{~s}, 3 \mathrm{H}) \cdot[\alpha]_{D}^{20}=22.9\left(\mathrm{c}=0.57, \mathrm{CH}_{2} \mathrm{Cl}_{2}\right)$.

\section{(S,E)-4-(3,7-dimethyl-3-vinylocta-1,6-dien-1-yl)phenol ((S)-Bakuchiol)}

To a solution of (S,E)-1-(3,7-dimethyl-3-vinylocta-1,6-dien-1-yl)-4-methoxybenzene (54 mg, $0.2 \mathrm{mmol})$ in $3 \mathrm{ml}$ of $\mathrm{Et}_{2} \mathrm{O}$ was added a soln. of $\mathrm{MeMgl}$ in $\mathrm{Et}_{2} \mathrm{O}(1.0 \mathrm{~mL}, 0.25 \mathrm{M})$. The solvent was removed under reduced pressure, and the residue was heated under Ar at 185 ${ }^{\circ} \mathrm{C}$ for $10 \mathrm{~min}$. The mixture was cooled to r.t., and the reaction was quenched with $1 \mathrm{ml}$ of sat. aq. $\mathrm{NH}_{4} \mathrm{Cl}$ soln. The product was extracted with $\mathrm{Et}_{2} \mathrm{O}$. The combined organic phase was washed with sat. aq. $\mathrm{NaCl}$ soln. and dried $\left(\mathrm{Na}_{2} \mathrm{SO}_{4}\right)$. The solvent was removed under reduced pressure, and the crude product was purified by column chromatography (silica gel; Petroleum ether : Ethyl acetate $=10: 1)$ to afford (S)-Bakuchiol ${ }^{[4]}$ in $80 \%$ yield. ${ }^{1} \mathrm{H}$ NMR $\left(400 \mathrm{MHz}, \mathrm{CDCl}_{3}\right) \delta 7.25(\mathrm{~d}, \mathrm{~J}=9.9 \mathrm{~Hz}, 2 \mathrm{H}), 6.77(\mathrm{~d}, \mathrm{~J}=8.5 \mathrm{~Hz}, 2 \mathrm{H}), 6.25(\mathrm{~d}, \mathrm{~J}=16.3 \mathrm{~Hz}$, $1 \mathrm{H}), 6.05(\mathrm{~d}, \mathrm{~J}=16.2 \mathrm{~Hz}, 1 \mathrm{H}), 5.88(\mathrm{dd}, \mathrm{J}=17.4,10.8 \mathrm{~Hz}, 1 \mathrm{H}), 5.11(\mathrm{~d}, \mathrm{~J}=7.0 \mathrm{~Hz}, 1 \mathrm{H})$, 5.02 (dd, J = 13.7, 9.2 Hz, 2H), 1.95 (dd, J = 16.2, $7.5 \mathrm{~Hz}, 2 \mathrm{H}$ ), 1.67 (s, 3H), 1.58 (s, 3H), 1.49 (dd, $\mathrm{J}=10.2,6.7 \mathrm{~Hz}, 2 \mathrm{H}), 1.19(\mathrm{~s}, 3 \mathrm{H}) .{ }^{13} \mathrm{C} \mathrm{NMR}\left(101 \mathrm{MHz}, \mathrm{CDCl}_{3}\right) \delta 154.7,145.9$, 135.8, 131.3, 130. 8, 127.3, 126.5, 124.8, 115.3, 111.9, 42.5, 41.3, 25.7, 23.3, 23.2, 17.6. $[\alpha]_{D}^{20}=10.9\left(\mathrm{c}=0.1, \mathrm{CH}_{2} \mathrm{Cl}_{2}\right)$.

\section{(6) Reference}

1. (a) Gerard, A. P.; Giorgio C.; Stefania R. Bioorg. Med. Chem. 2003, 11, 4015. (b) Zhang, P.; Le, Hai.; Kyne, R. E.; Morken, P. J. J. Am. Chem. Soc. 2014, 136, 17918. (c) Eun-Taek Oh; Hee Jin Kim; Jung Taek Oh; Liang S.; Inkyun Yun; Kyunggu Nam; Jae-Hong Min; Joon Woo Kim; Sangho Koo. Eur. J. Org. Chem. 2012, 4954.

2. Guo, H.; Dong, C.-G.; Kim, D.-S.; Urabe, D.; Wang, J.; Kim, J. T.; Liu, X.; Sasaki, T.; Kishi, Y. J. Am. Chem. Soc. 2009, 131, 15387.

3. (a) Denmark, S. E.; Fu, J. Org. Lett. 2002, 4, 1951. (b) Denmark, S. R.; Fu, J.; Lawler, M. J. J. Org. Chem., 2006, 71, 1523. (c) Denmark, S. R.; Fu, J.; Coe, D.M.; Su, X.; Pratt, N. E.; Griedel, B. D. J. Org. Chem. 2006, 71, 1513. (d) Denmark, S. E.; Fu, J. J. Am. Chem. Soc. 2001, 123, 9488.

4. (a) Du, X-L.; Chen, H-L.; Feng, H-J.; Li, Y-C. Helv. Chim. Acta. 2008, 91, 371. (b) Shuki, A.; Yasuo, B. J. Chem. Soc., Perkin Trans. 1, 1991, 10, 2395. 
3. Copies of NMR specta and HPLC chromatographs

L7-1 ${ }^{1} \mathrm{H}$

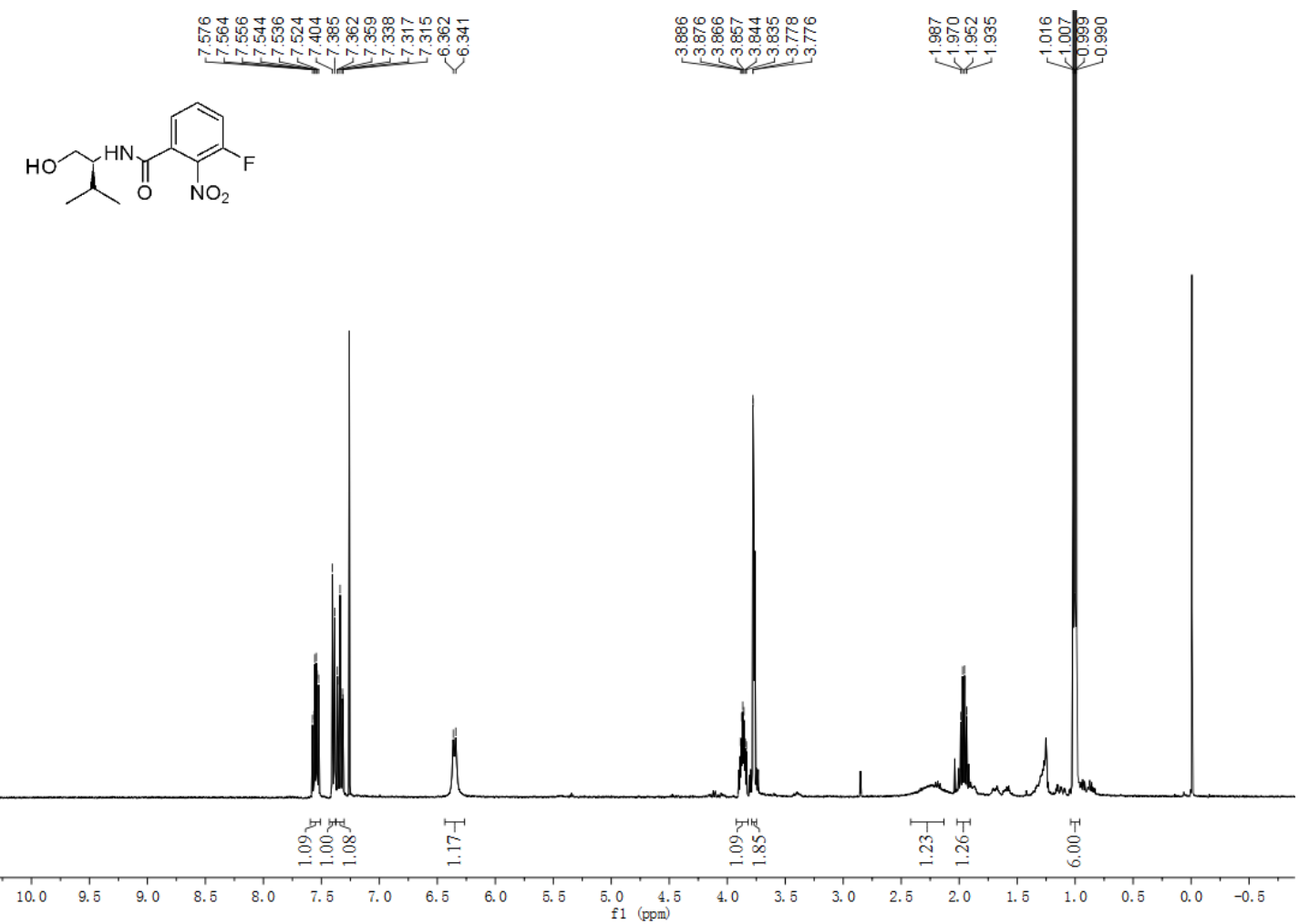

L7-1 ${ }^{13} \mathrm{C}$

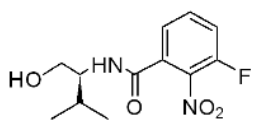

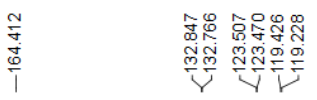

\begin{tabular}{ll}
$\overline{0}$ & \multirow{0}{0}{} \\
0 & 0 \\
0 & 1 \\
1 & 1
\end{tabular}

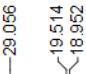
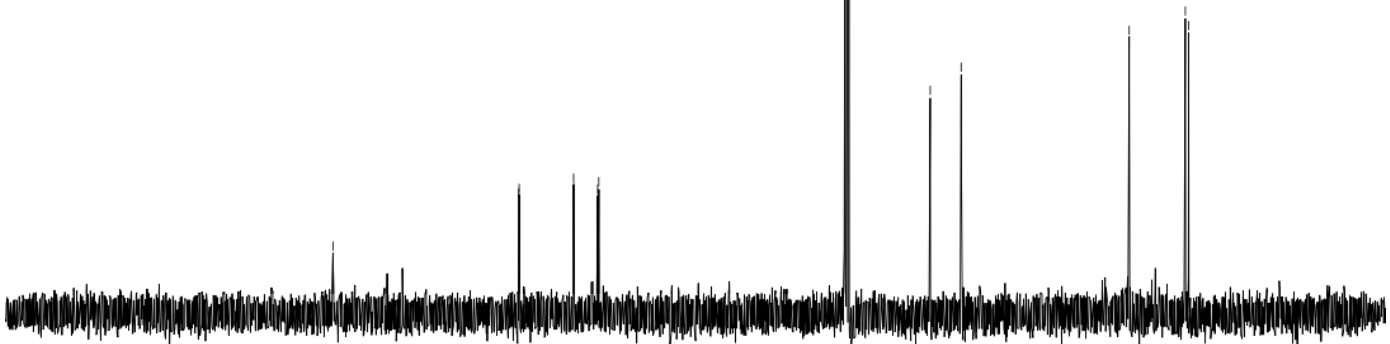

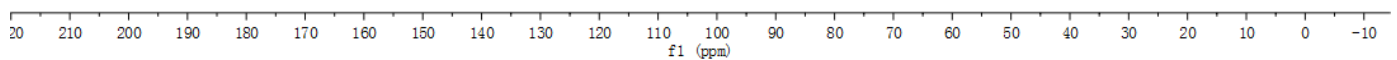


L7-1 ${ }^{19} \mathrm{~F}$

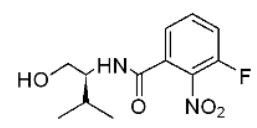

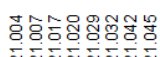

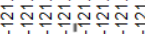

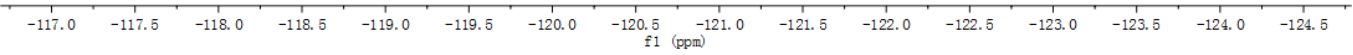

L7-2 ${ }^{1} \mathrm{H}$

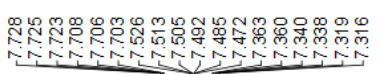

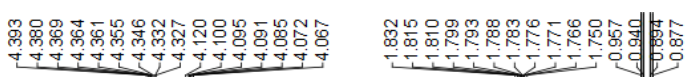

$\sum_{-\mathrm{N}}^{\mathrm{O}} \mathrm{NO}_{2}$

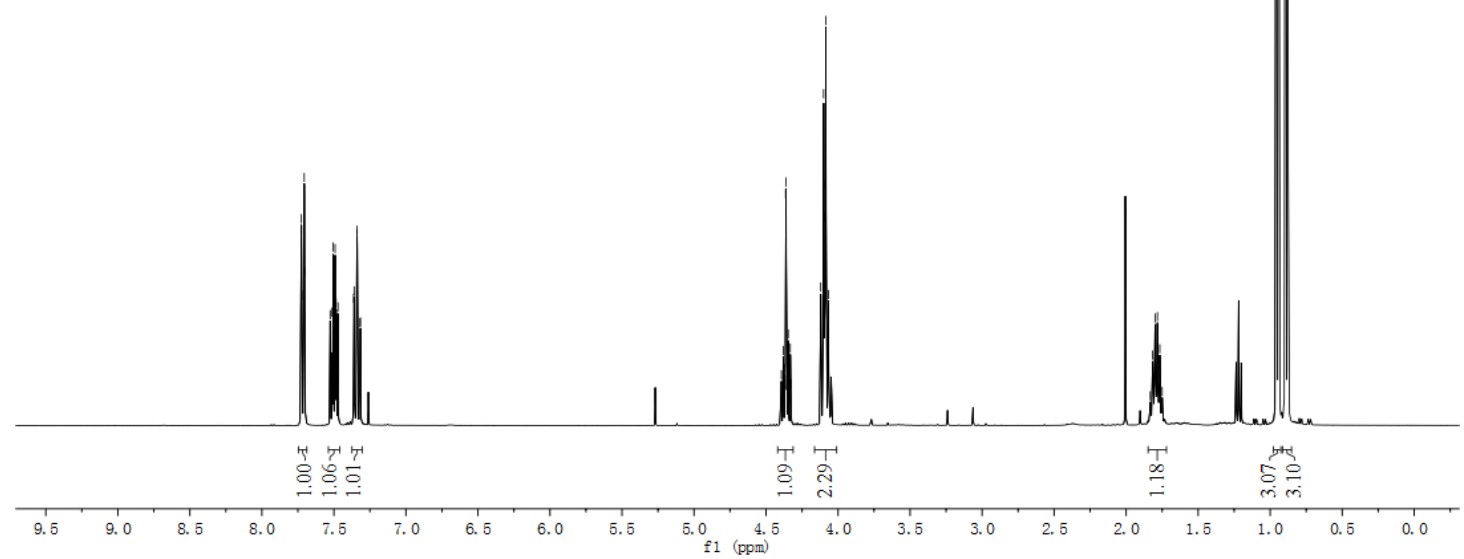



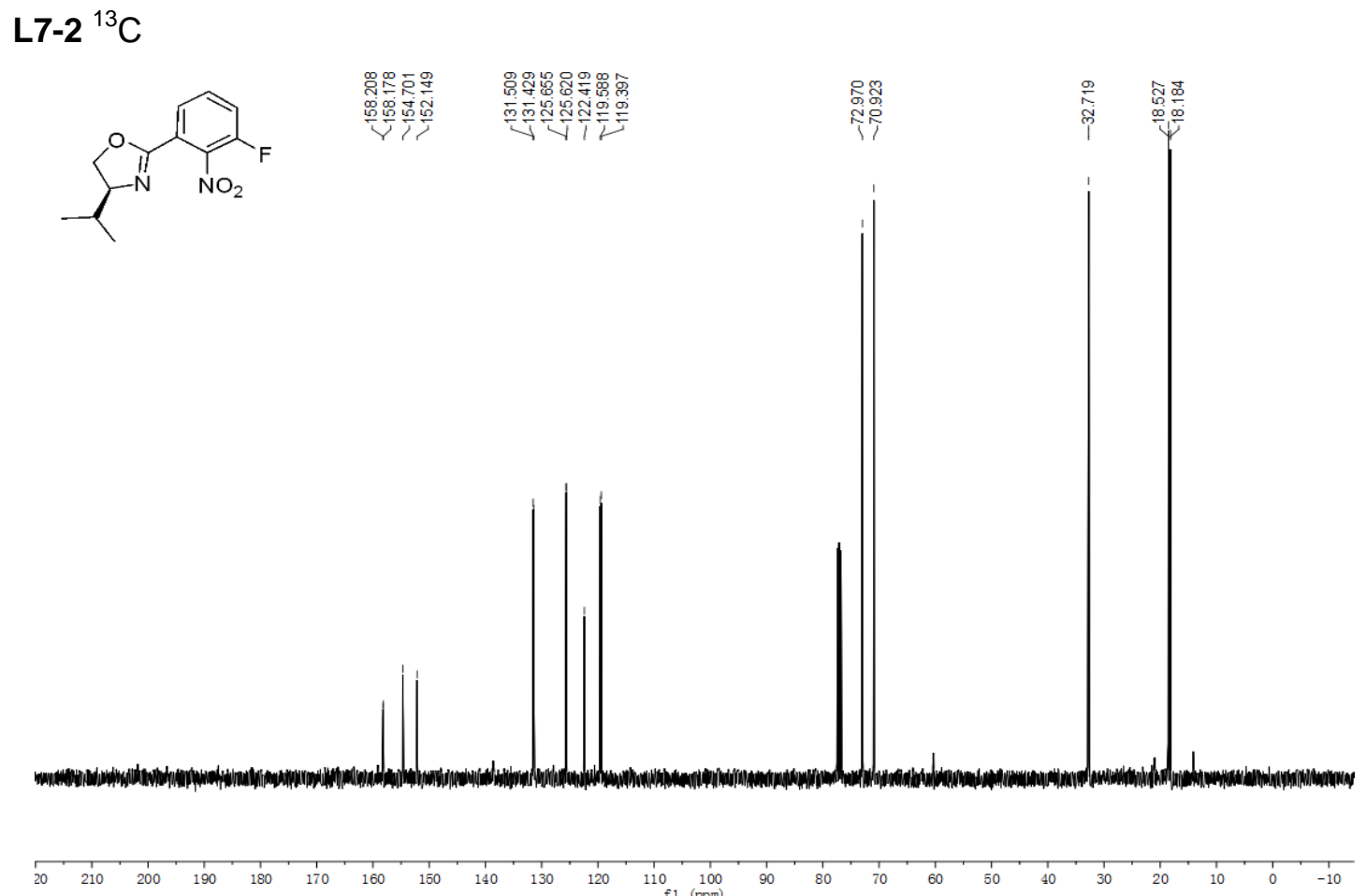

L7-2 ${ }^{19} \mathrm{~F}$

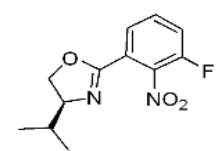

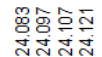

문둔

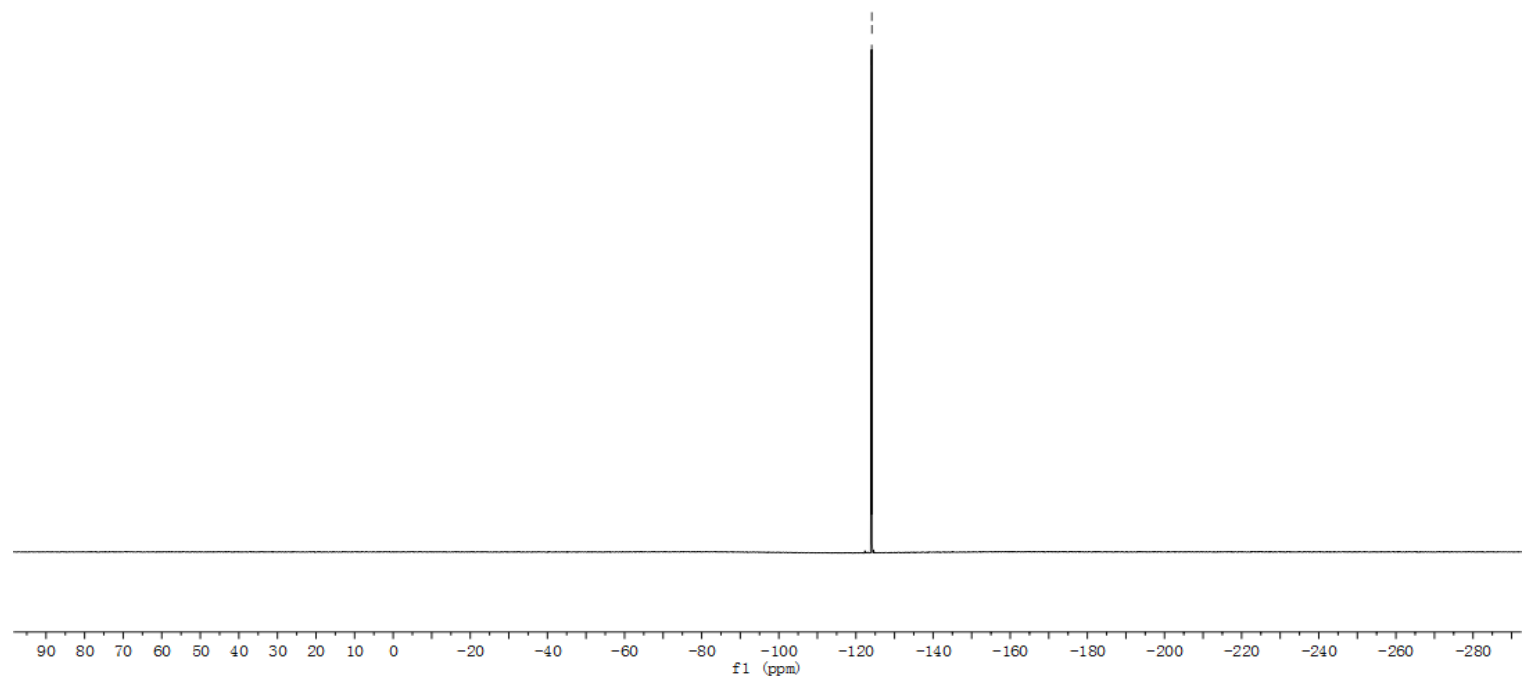


L7-3 ${ }^{1} \mathrm{H}$

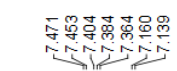

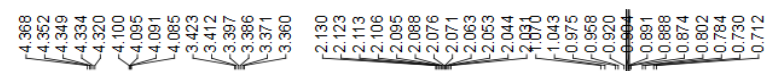

$\int_{-N}^{n}$
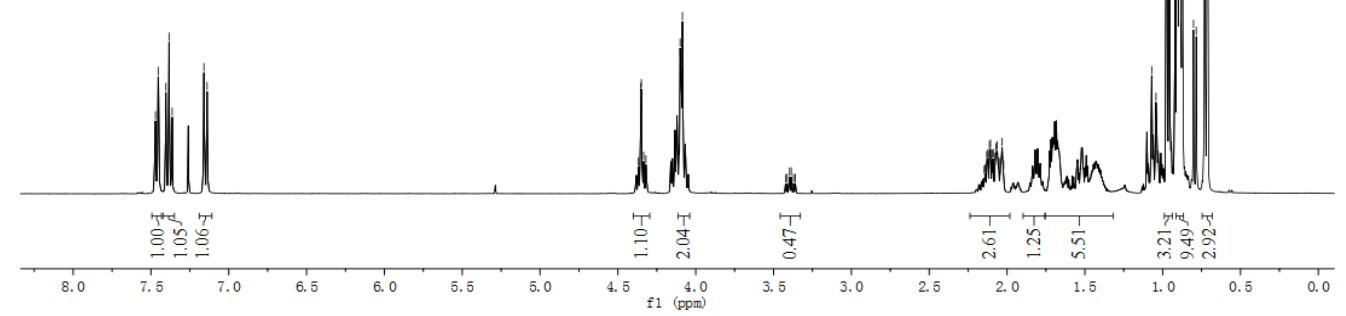

L7-3 ${ }^{13} \mathrm{C}$

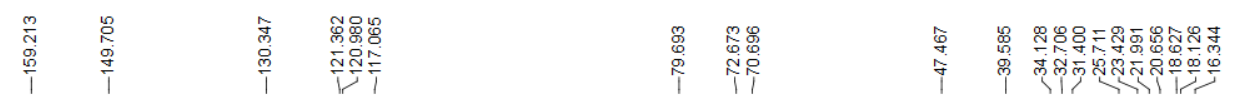<smiles>CC1CCC(C(C)C)C(Oc2cccc(C3=NC(C(C)C)CO3)c2[N+](=O)[O-])C1</smiles>

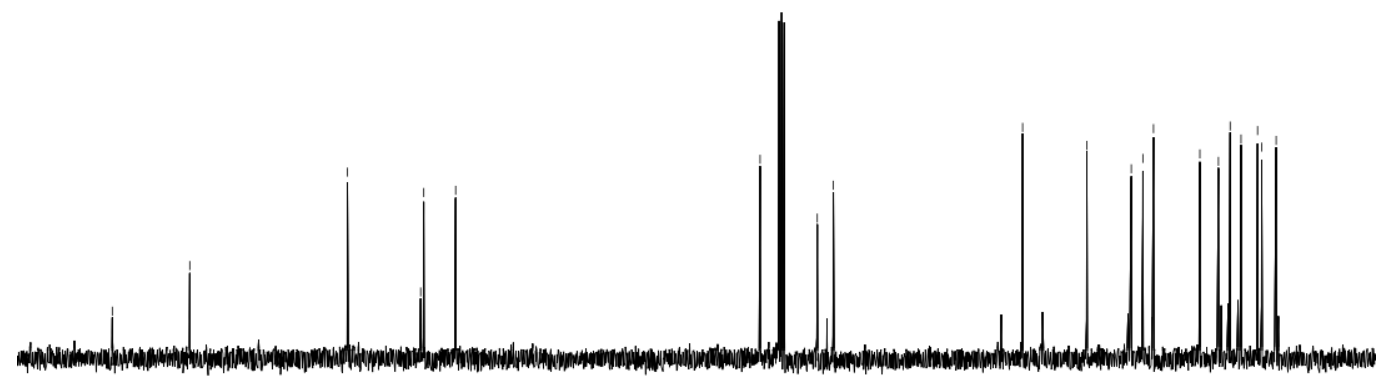

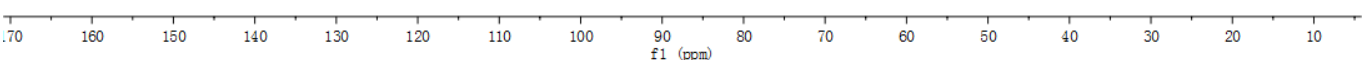


L7-4 ${ }^{1} \mathrm{H}$

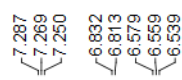

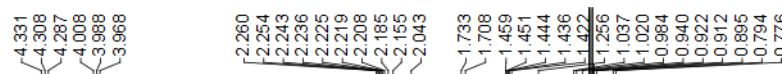
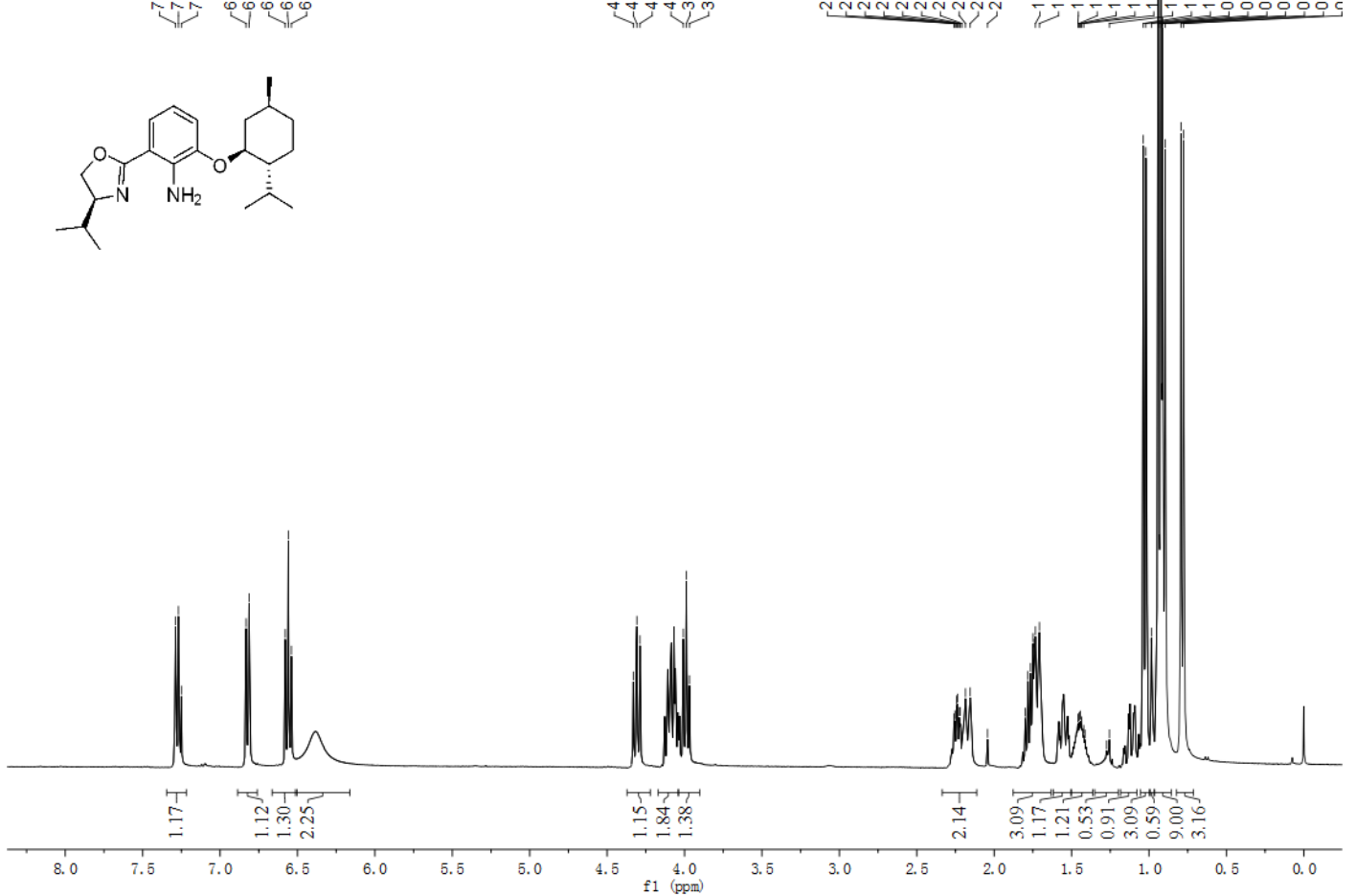

\section{L6 ${ }^{1} \mathrm{H}$}

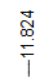

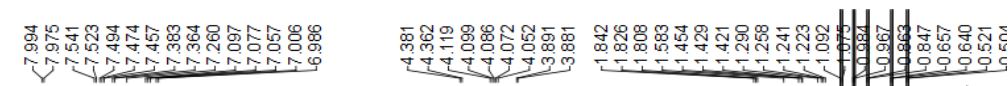

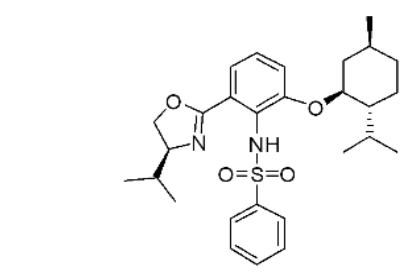

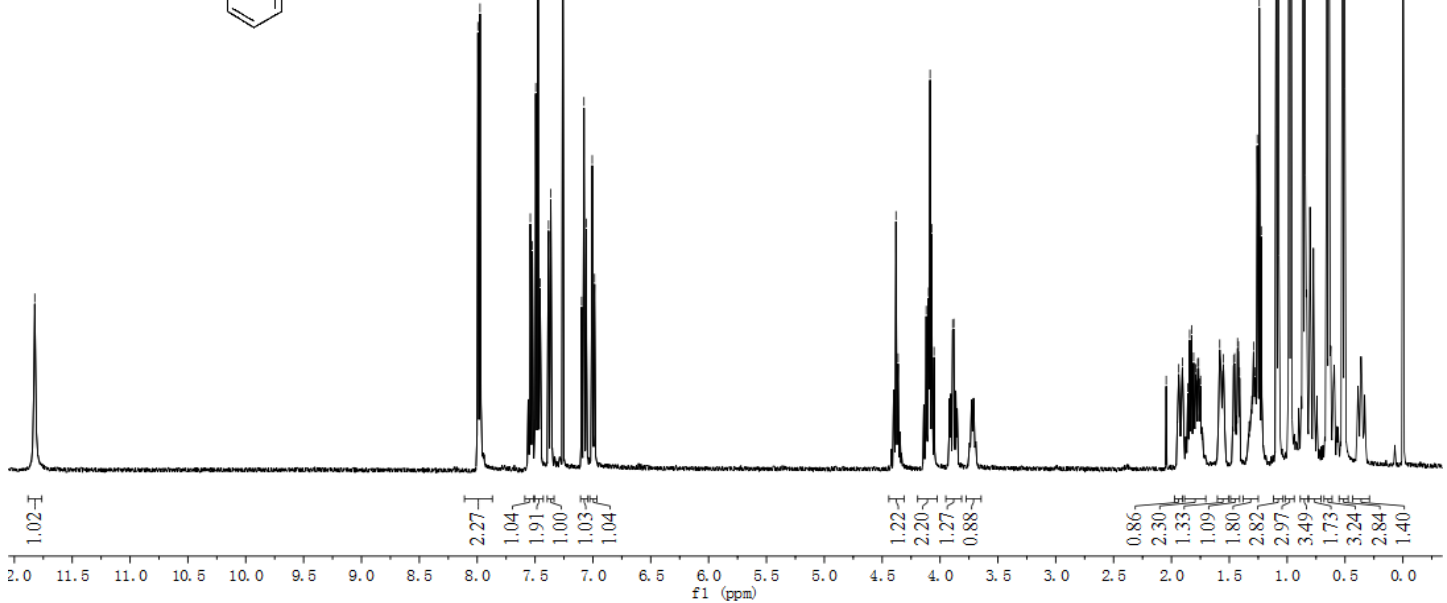


L7 ${ }^{1} \mathrm{H}$

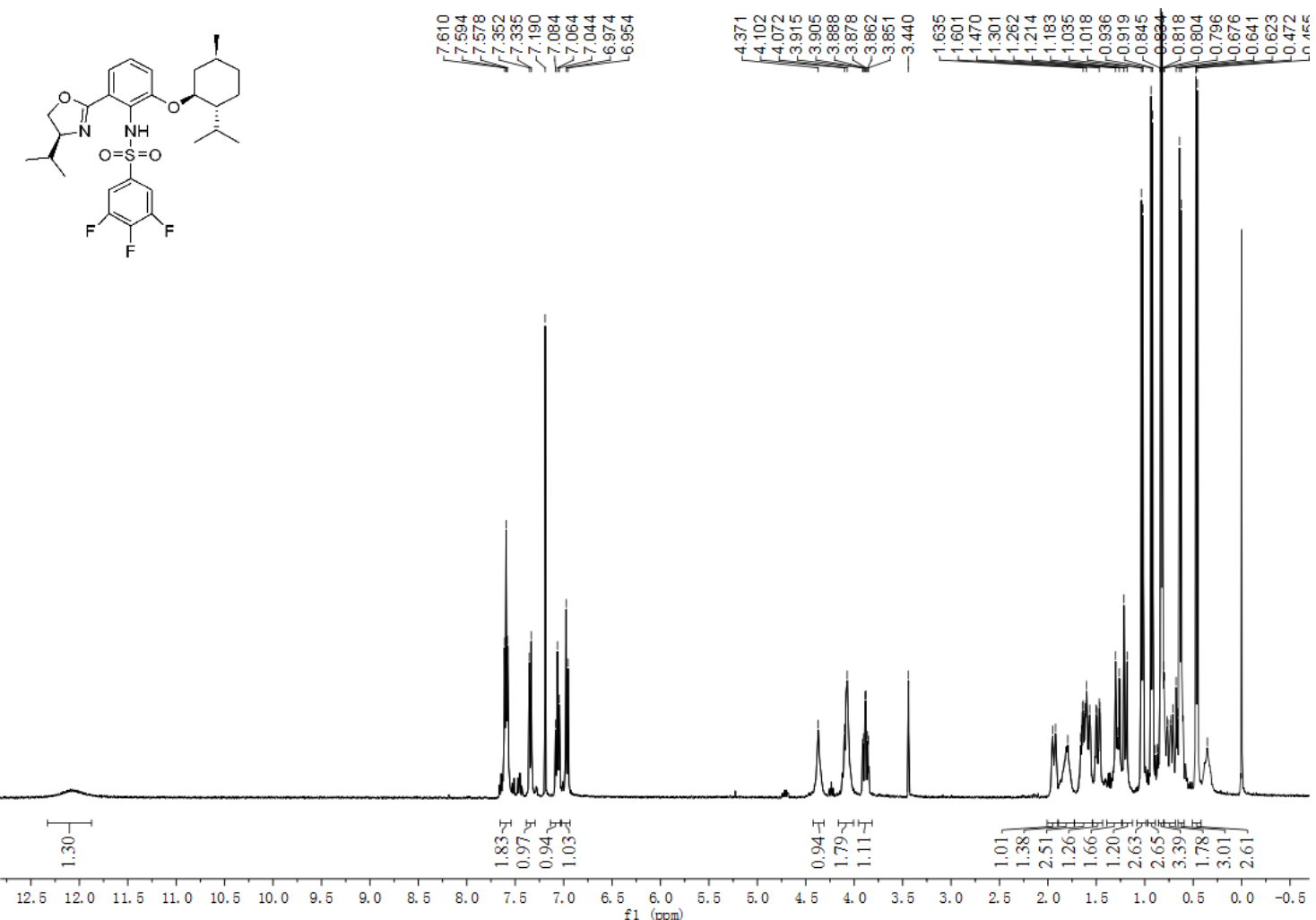

L7 ${ }^{13} \mathrm{C}$

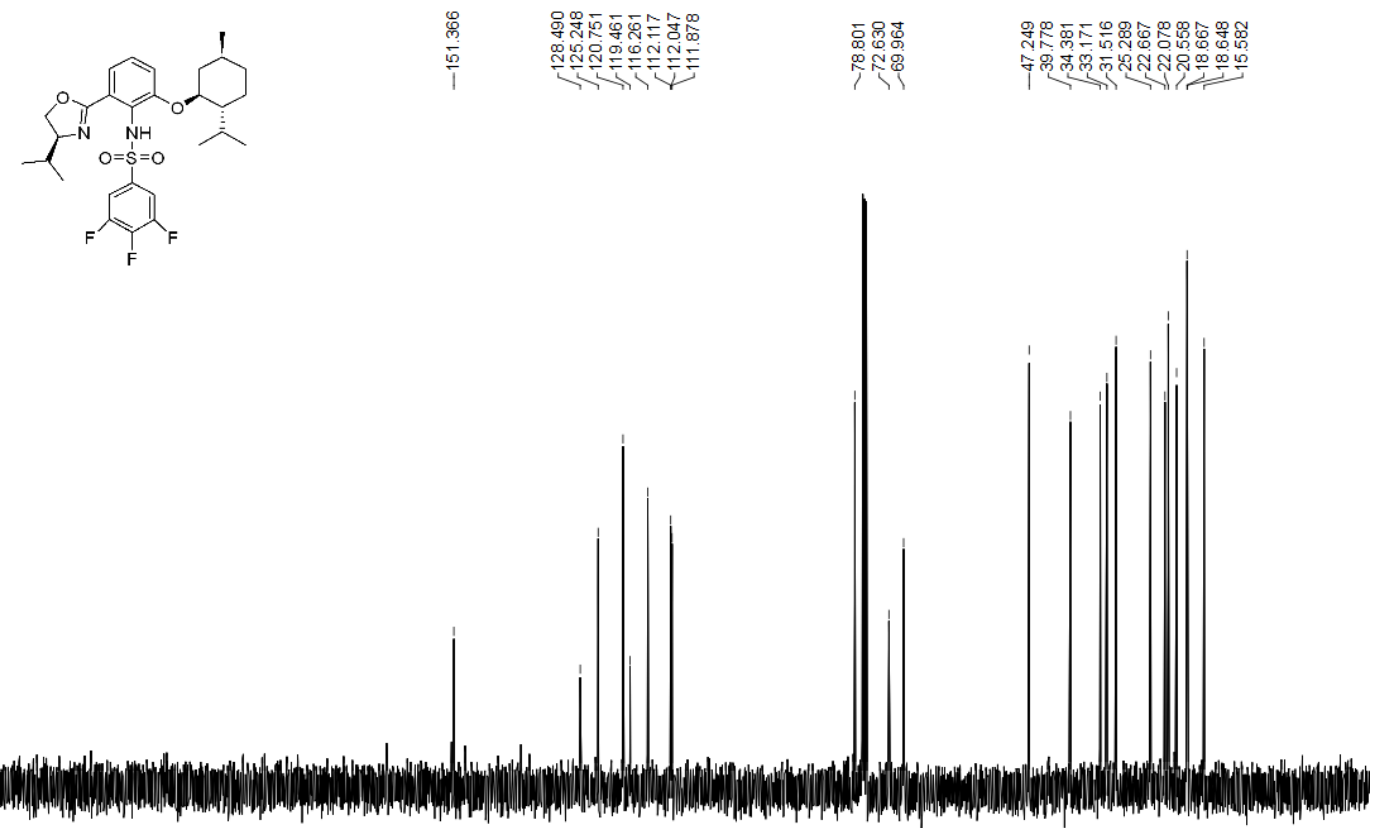

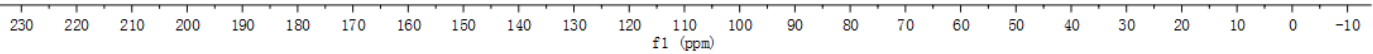



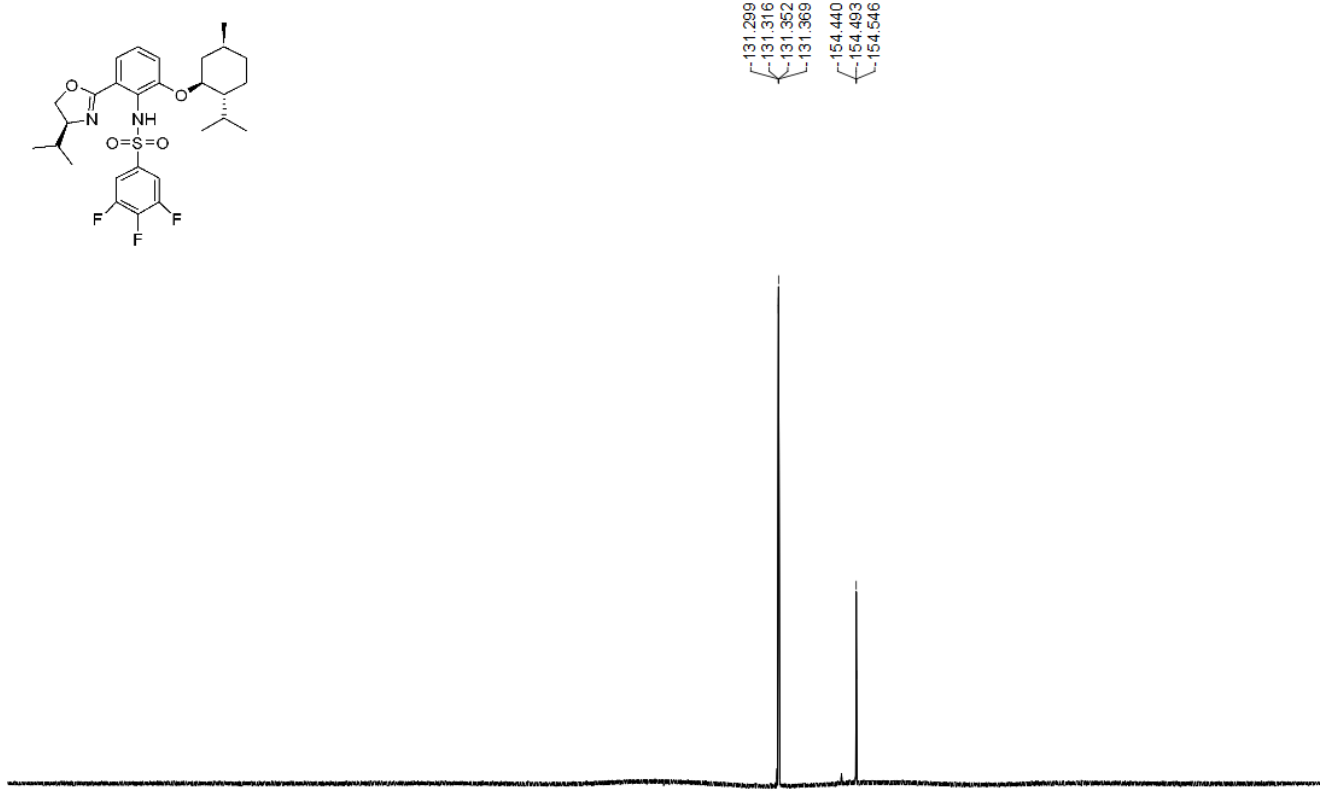

L8 ${ }^{1} \mathrm{H}$

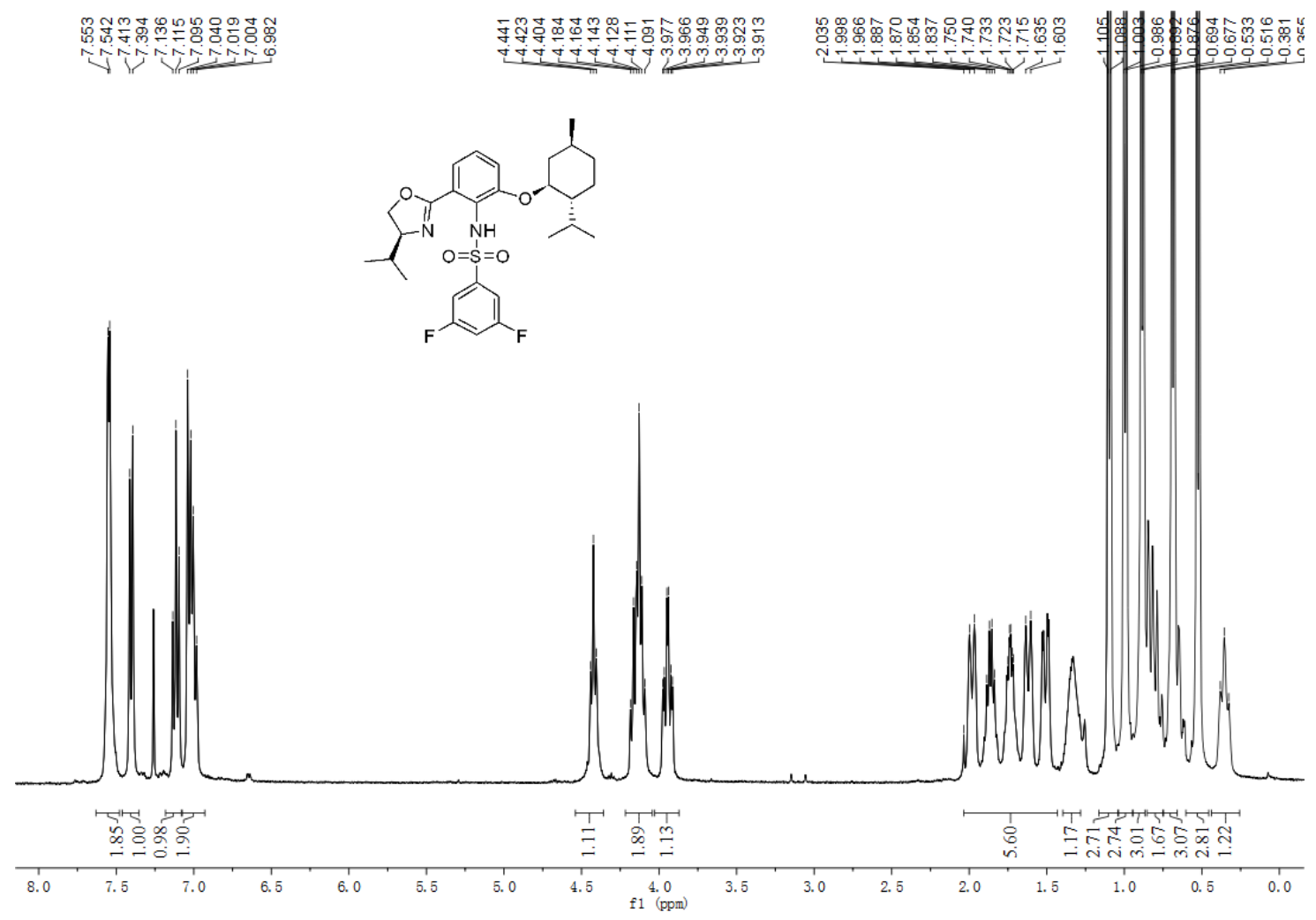


L8 ${ }^{13} \mathrm{C}$
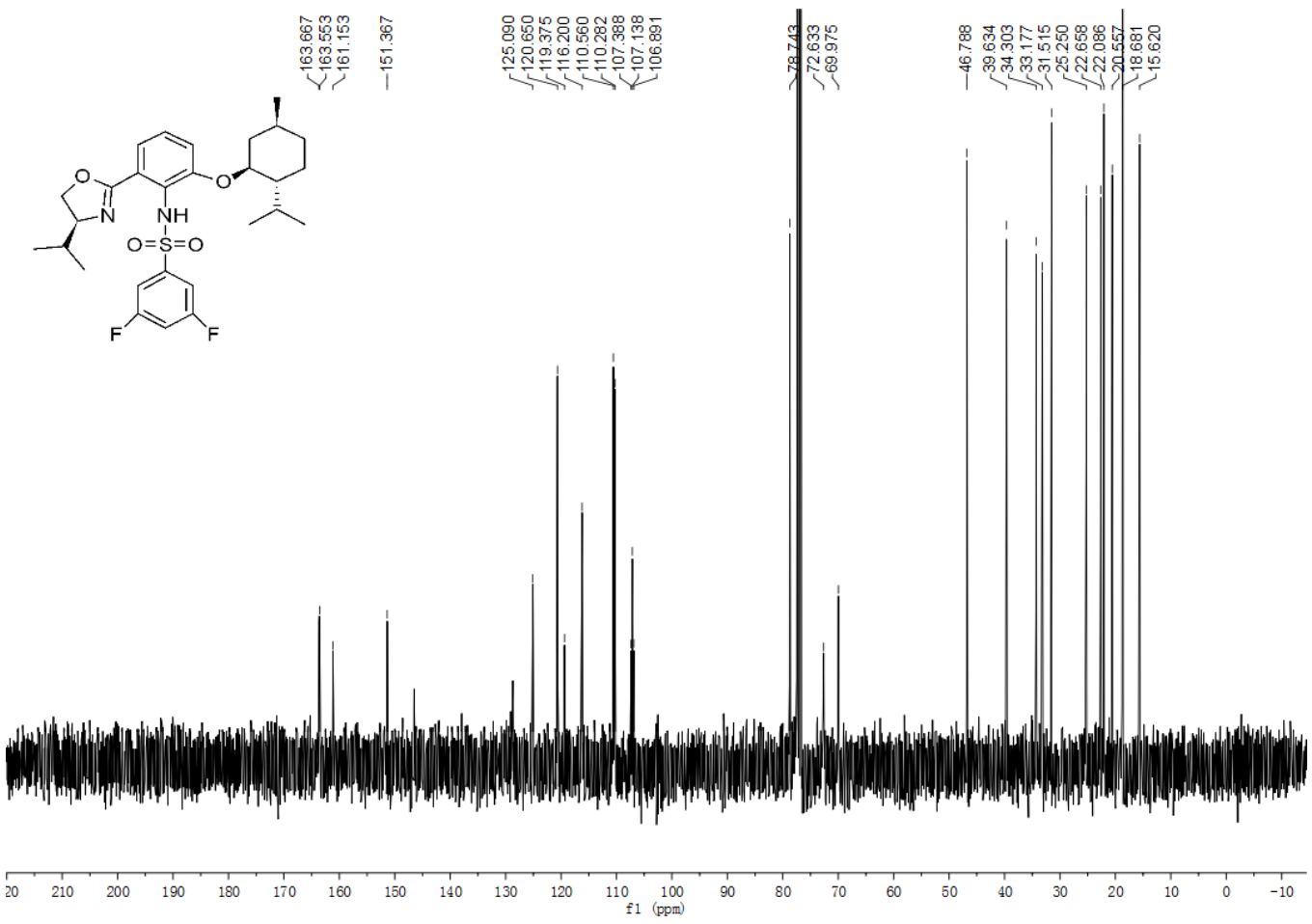

L9 ${ }^{1} \mathrm{H}$

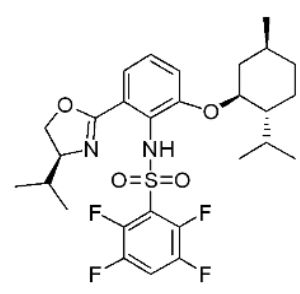

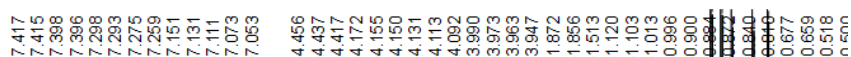

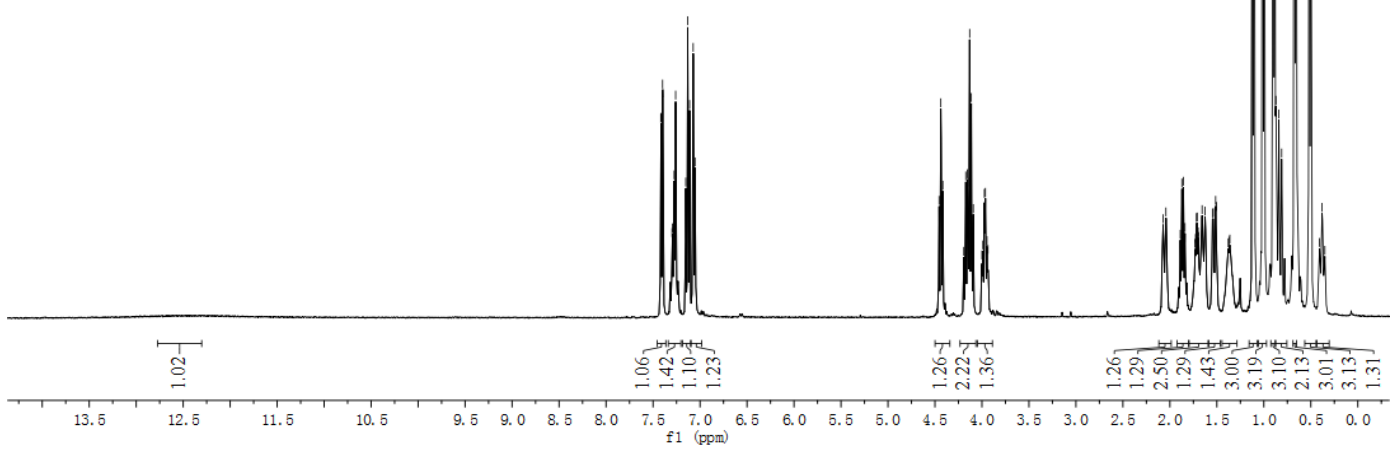


L9 ${ }^{13} \mathrm{C}$

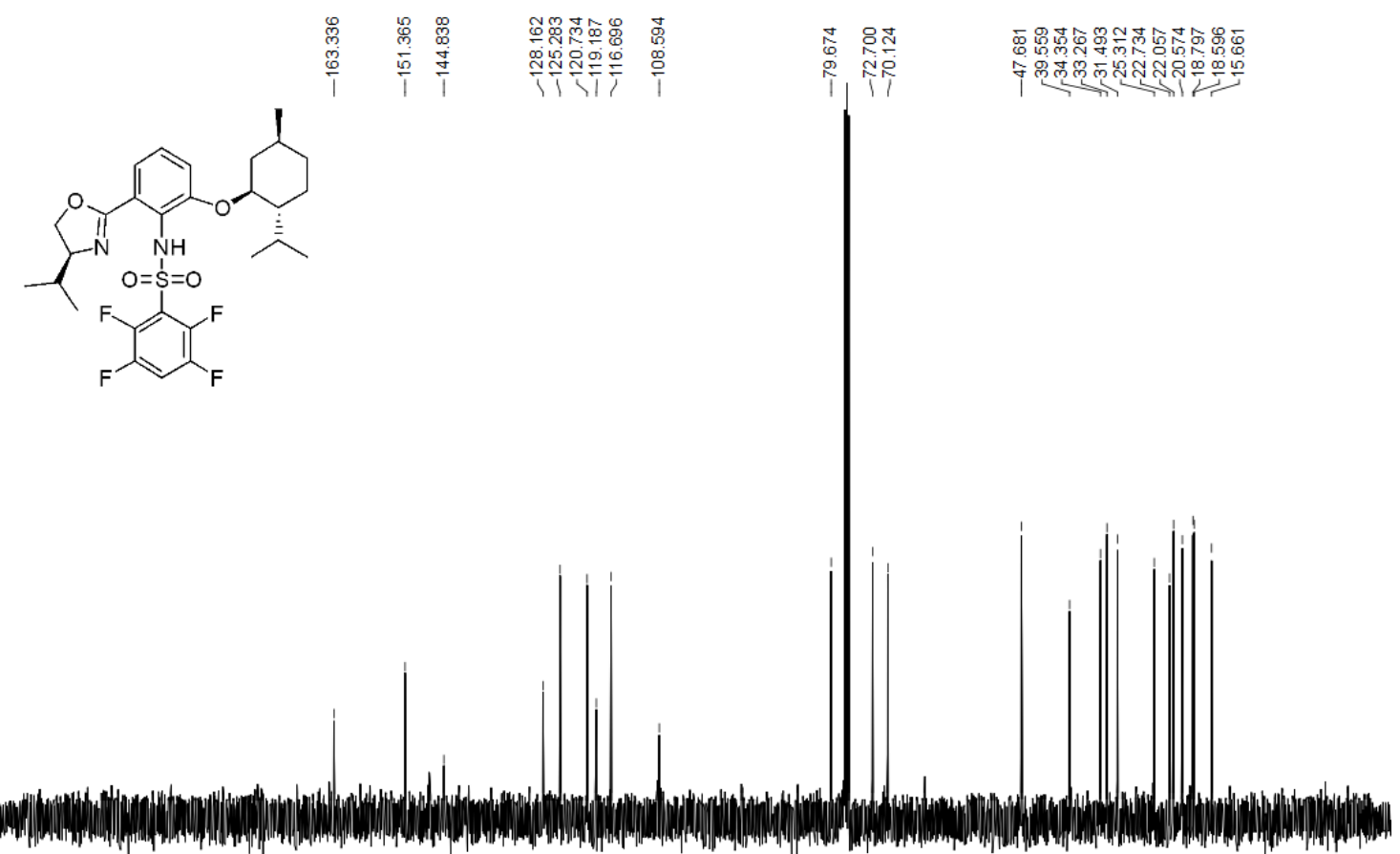

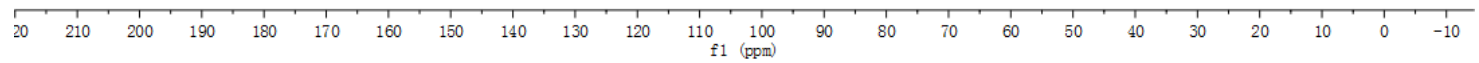

L10 ${ }^{1} \mathrm{H}$

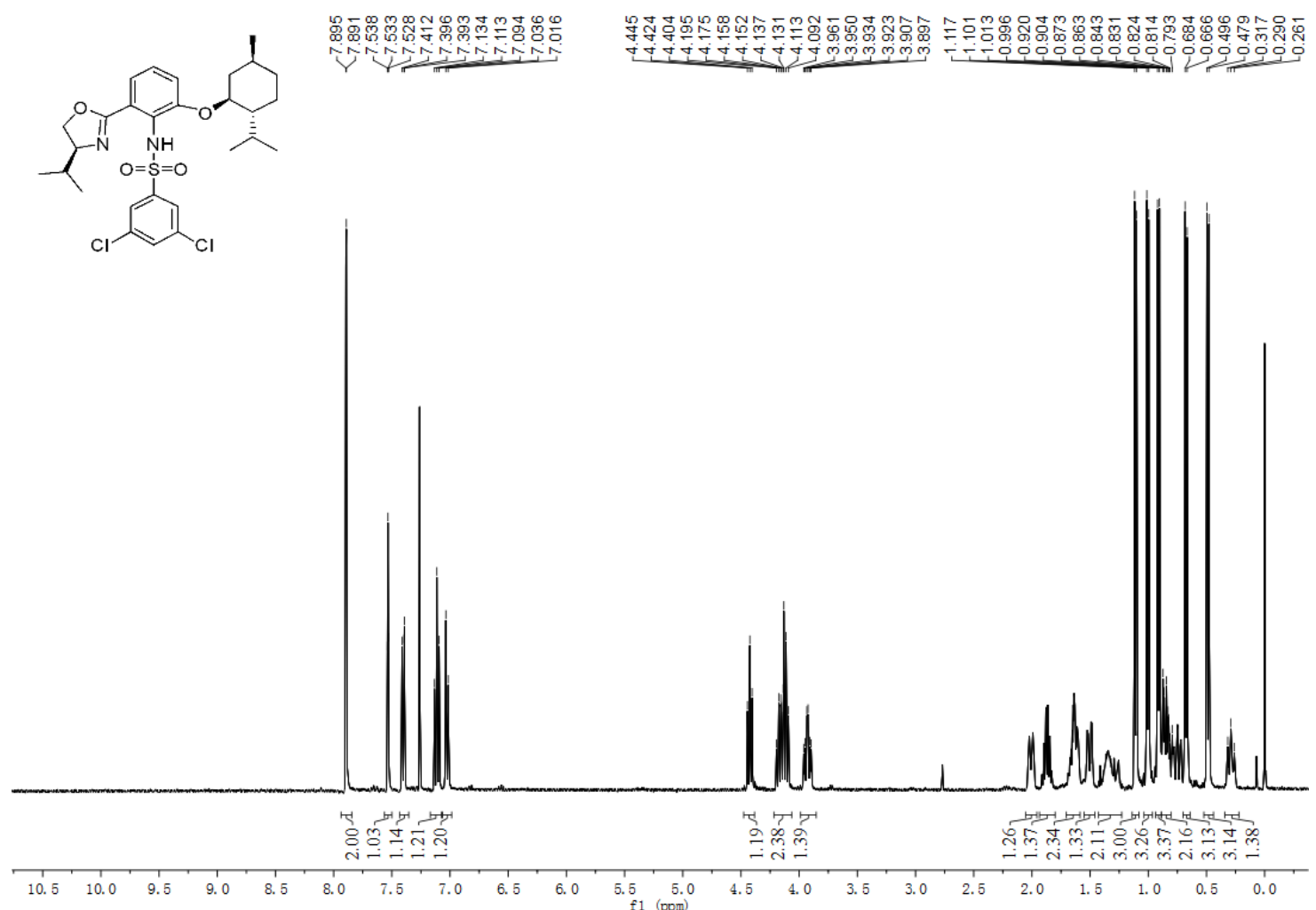



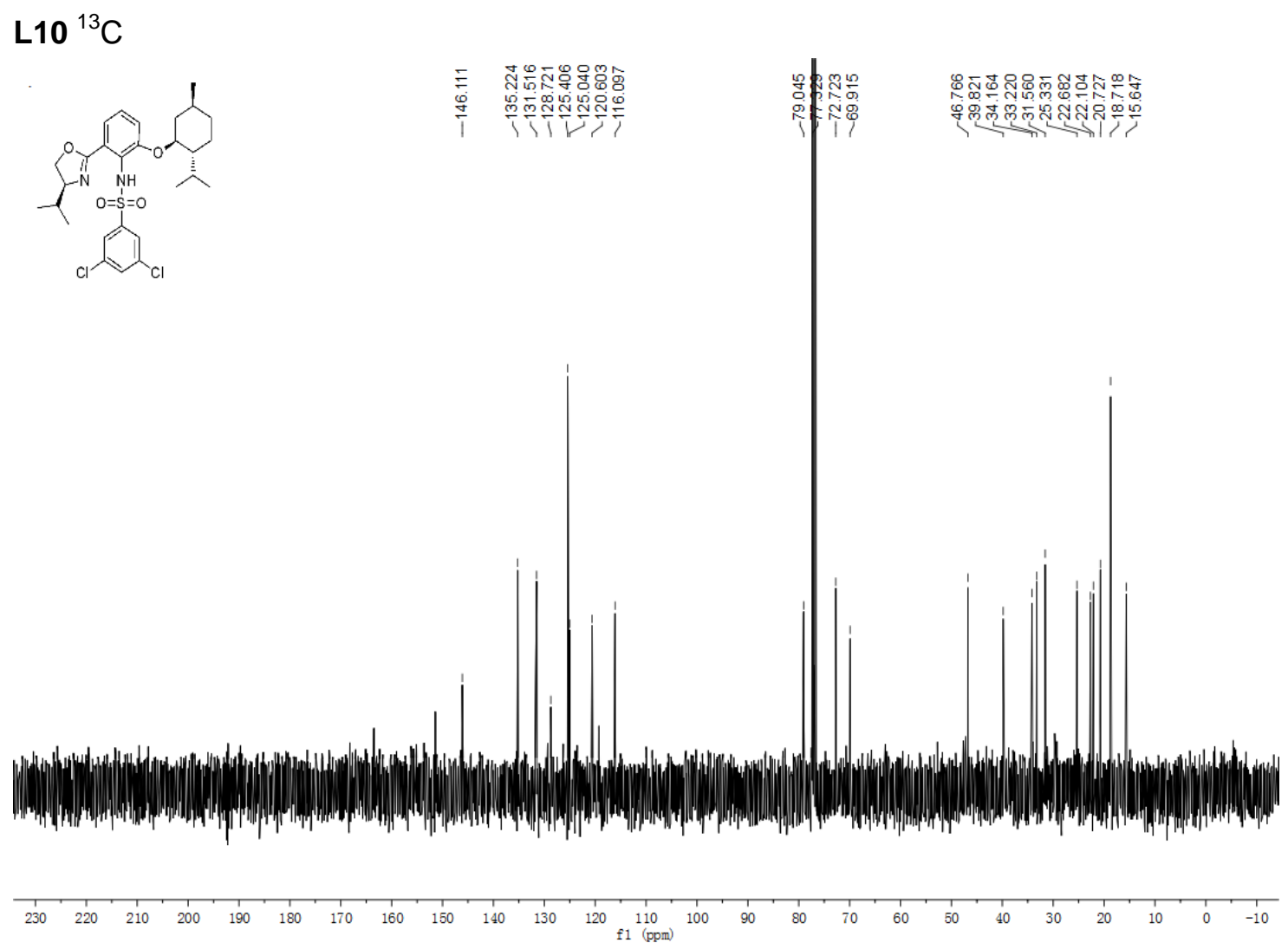

L11 ${ }^{1} \mathrm{H}$

$\stackrel{\substack{\infty \\ i}}{\stackrel{\infty}{i}}$

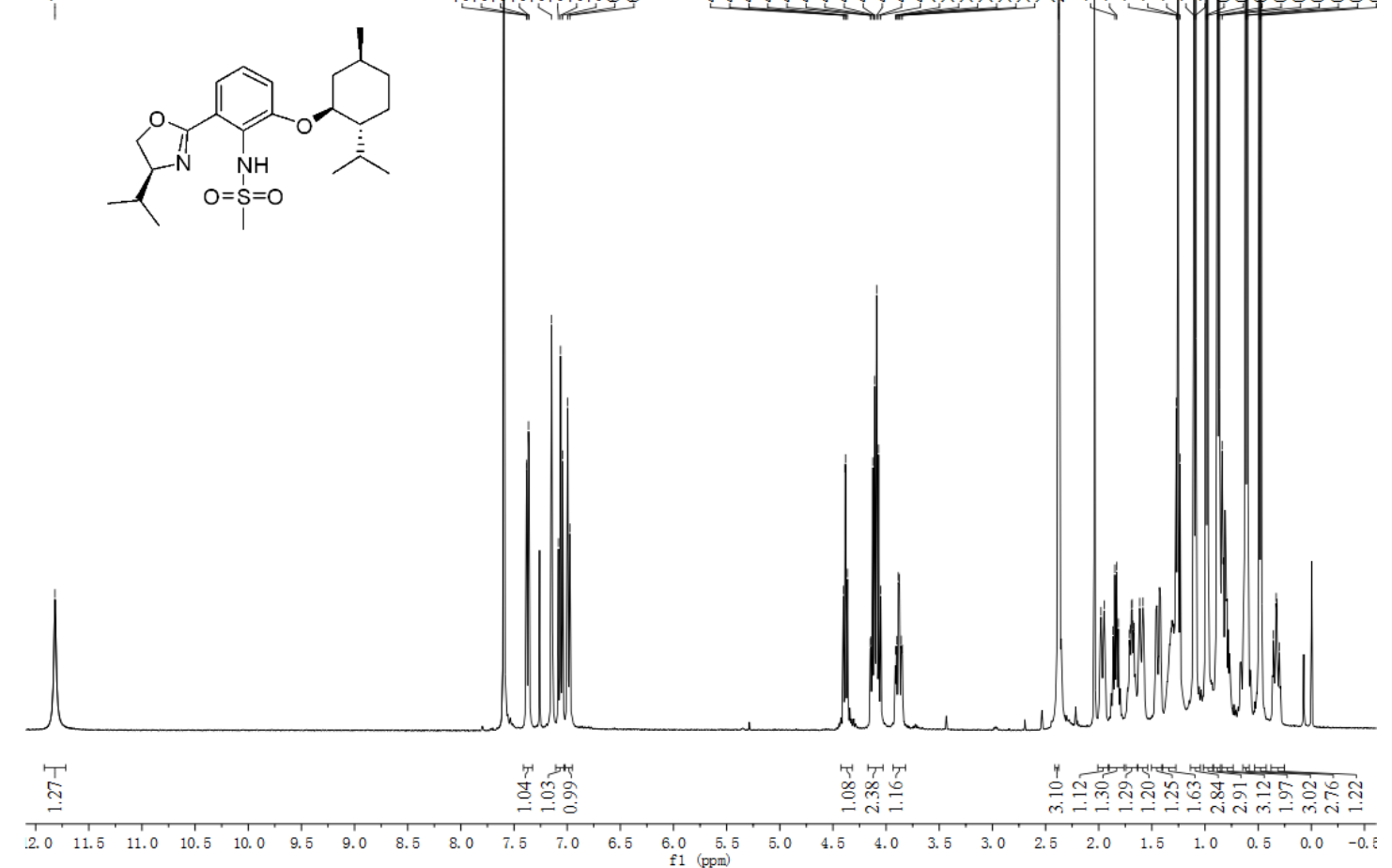

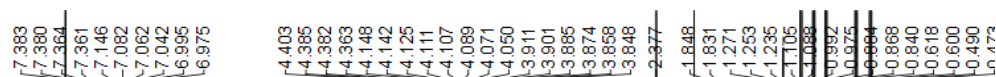


L11 ${ }^{13} \mathrm{C}$
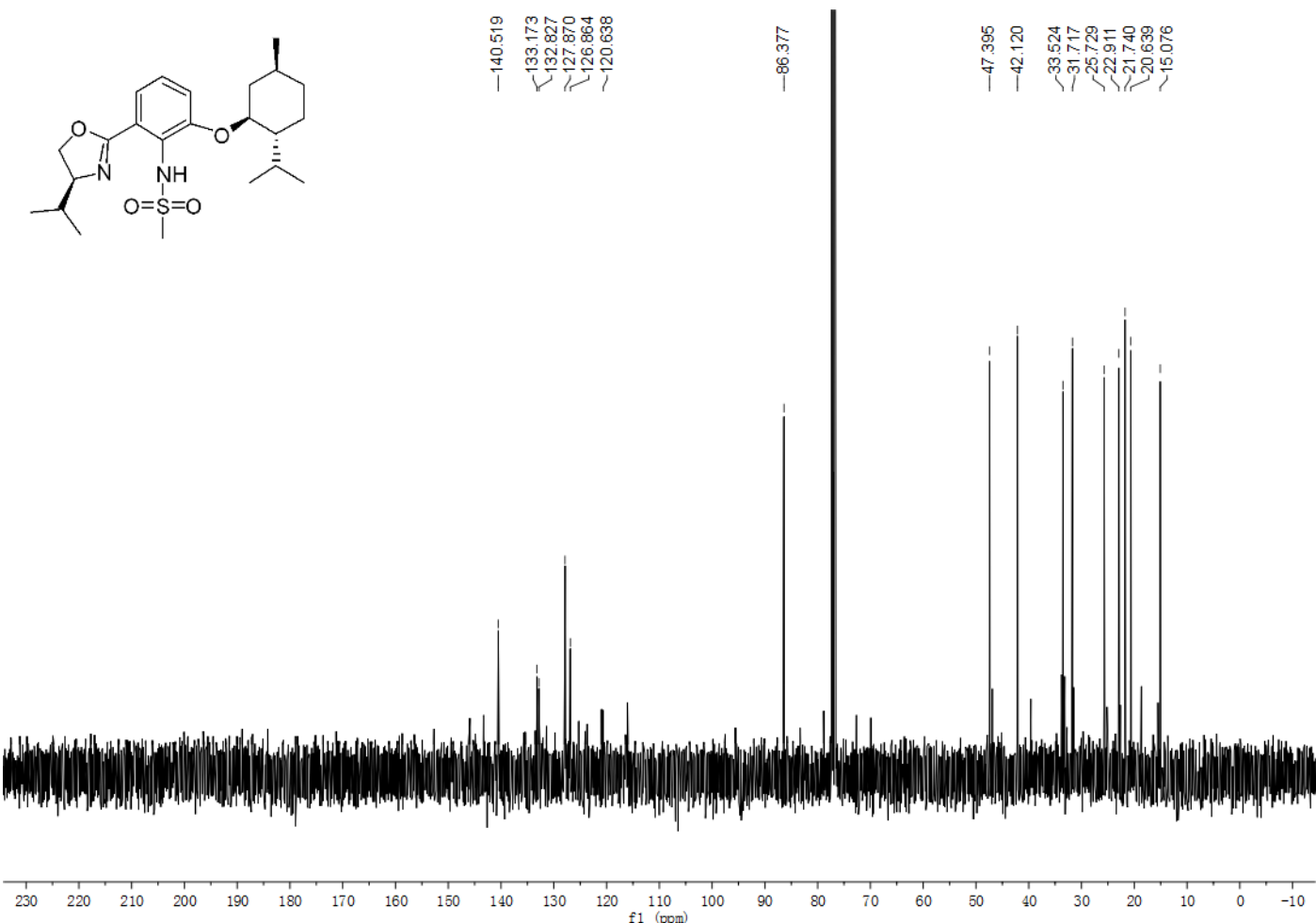

2a ${ }^{1} \mathrm{H}$
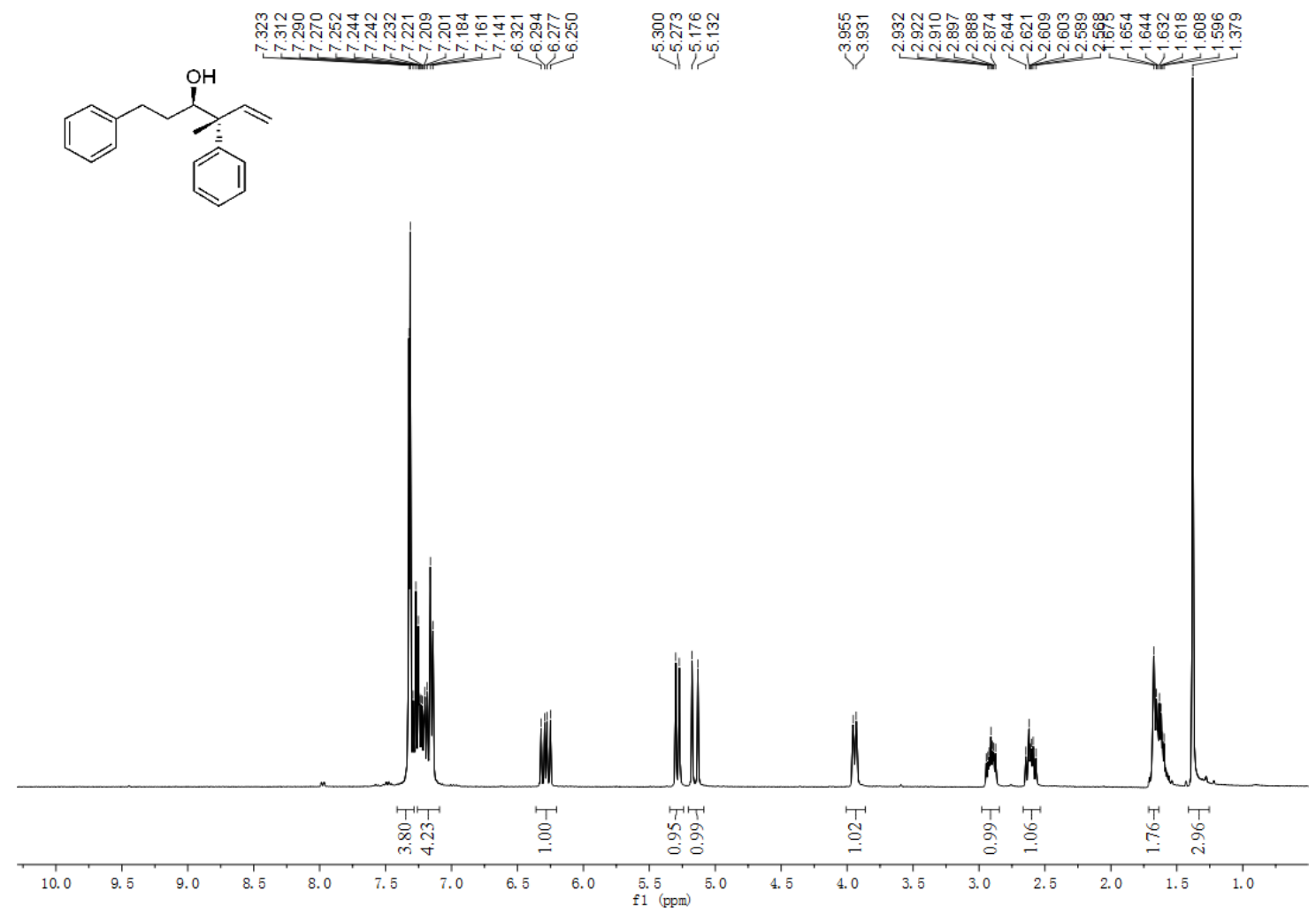
$2 a{ }^{13} \mathrm{C}$

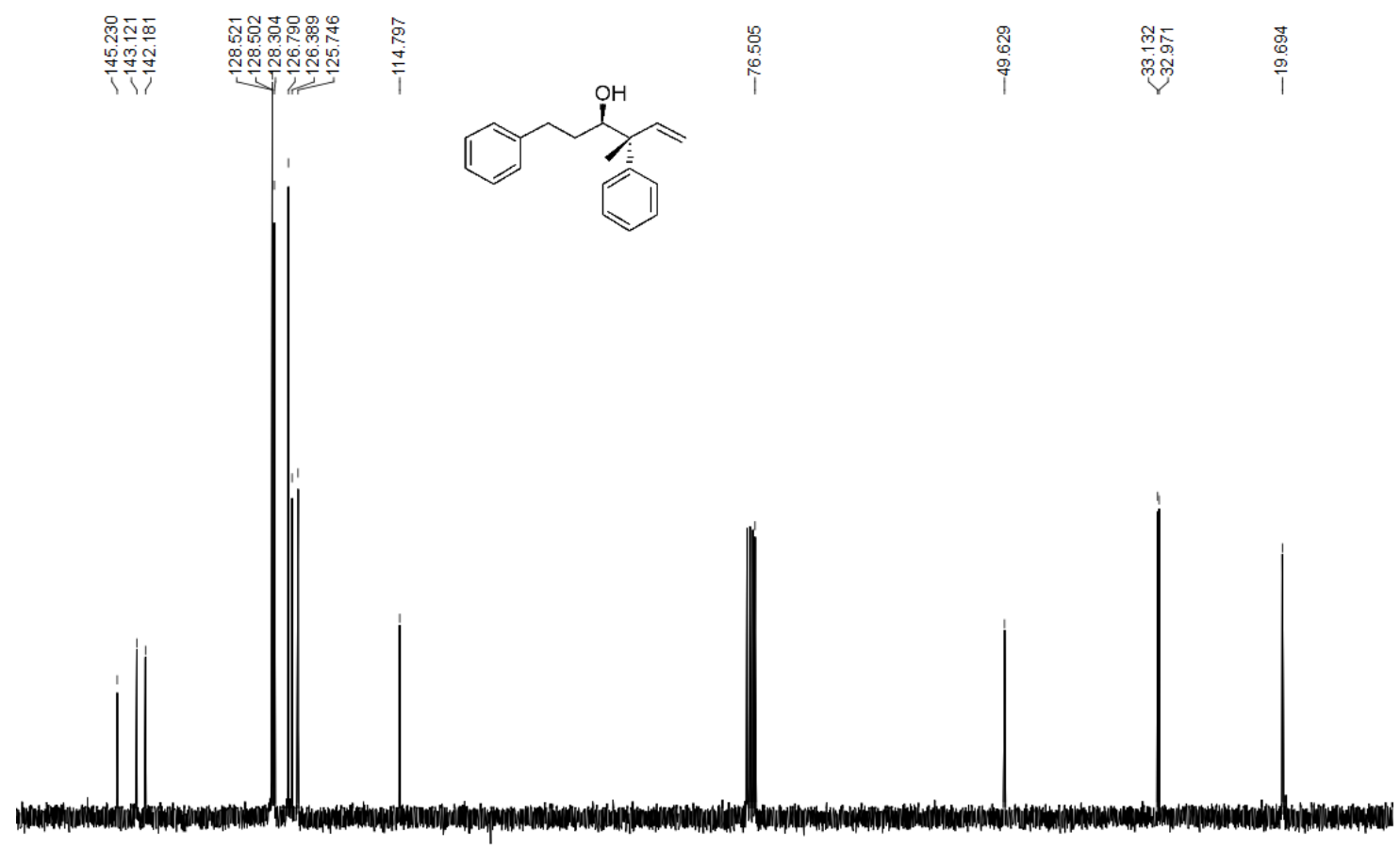

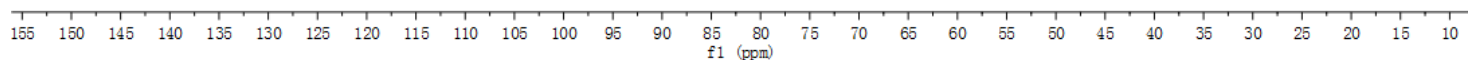

2a HPLC (racemic)

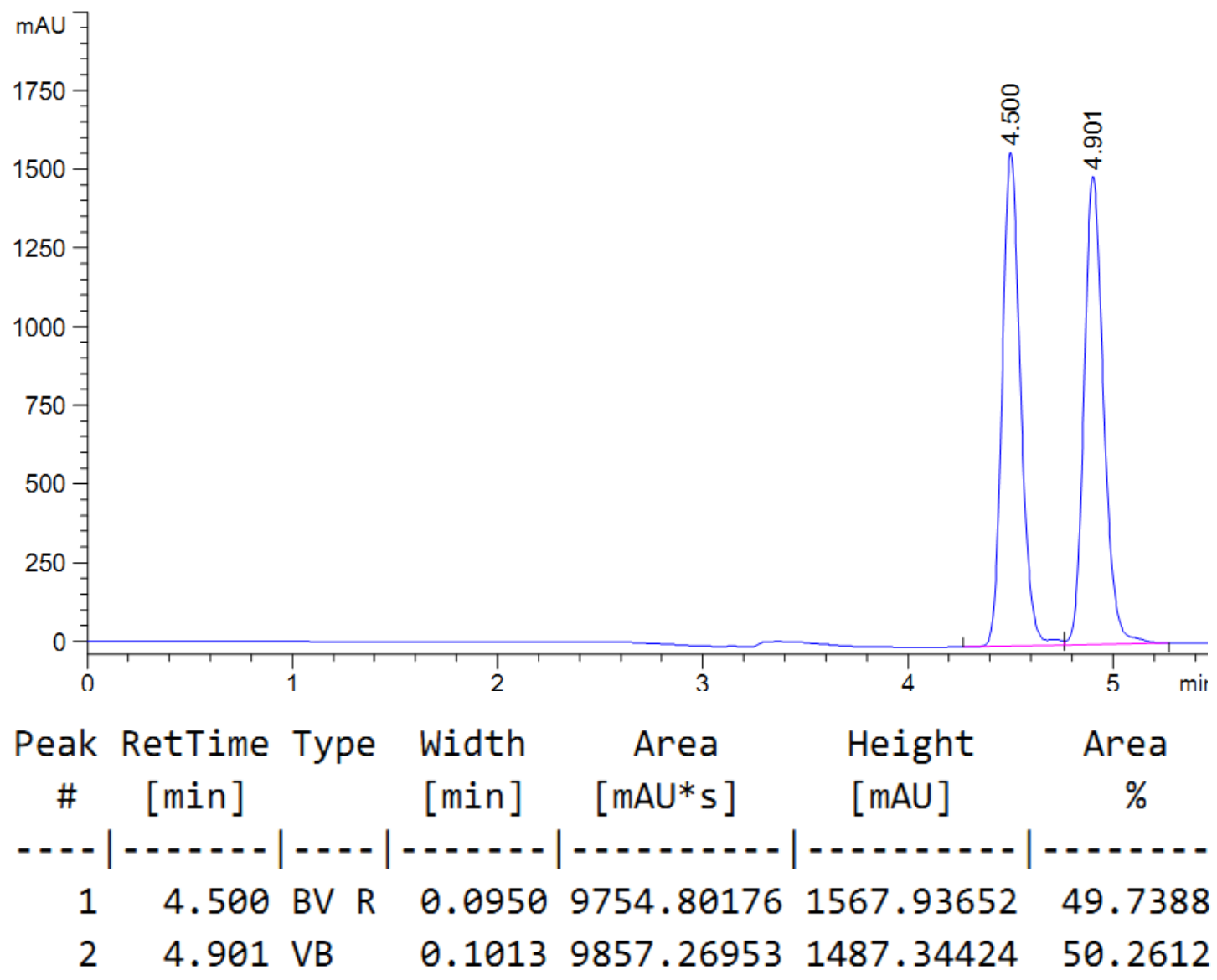


2a HPLC (91\% ee)

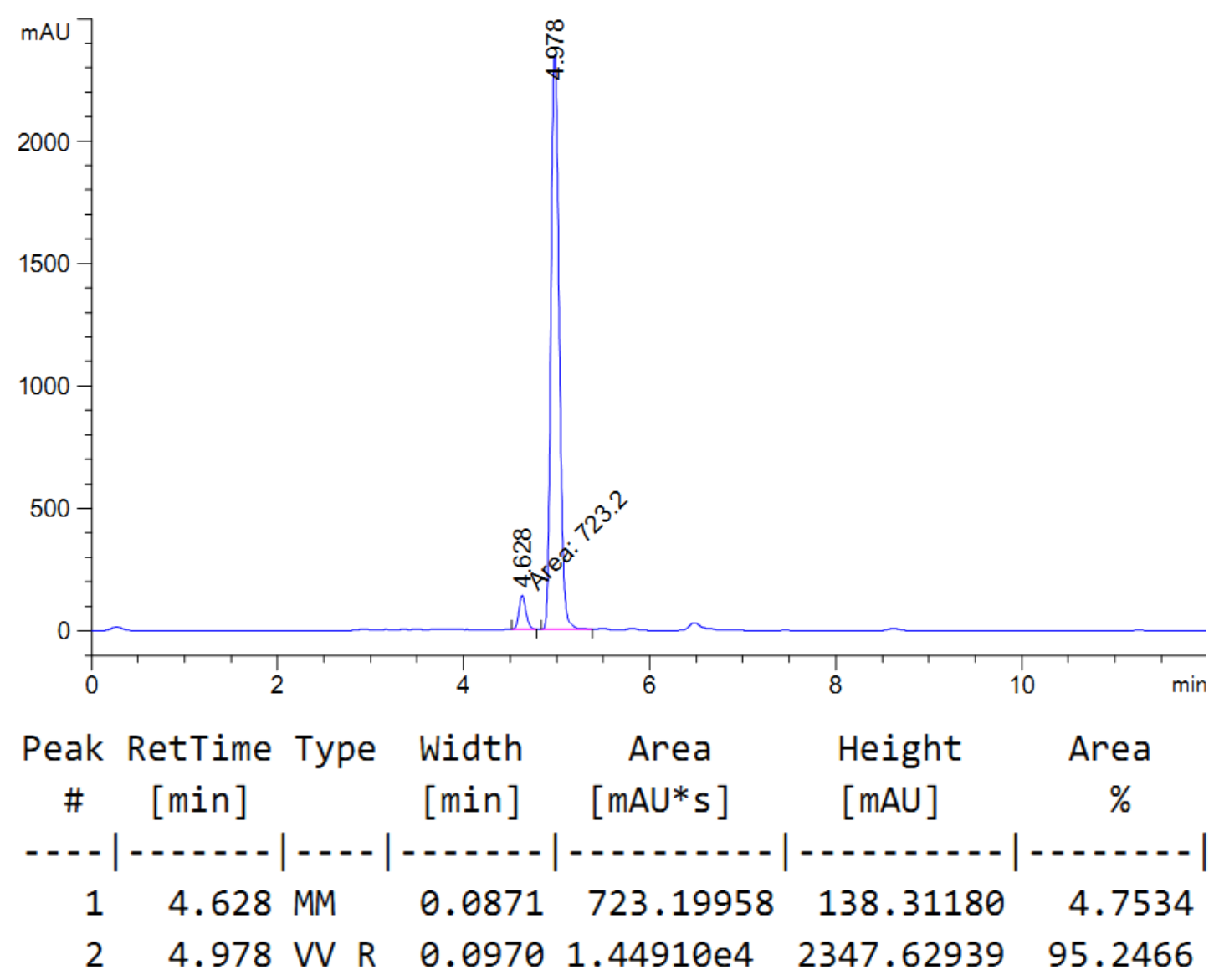

$2 b^{1} \mathrm{H}$

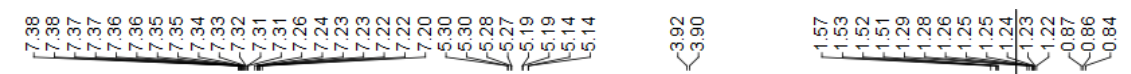<smiles>C=CC(C)(c1ccccc1)C(O)CCCCCC</smiles>

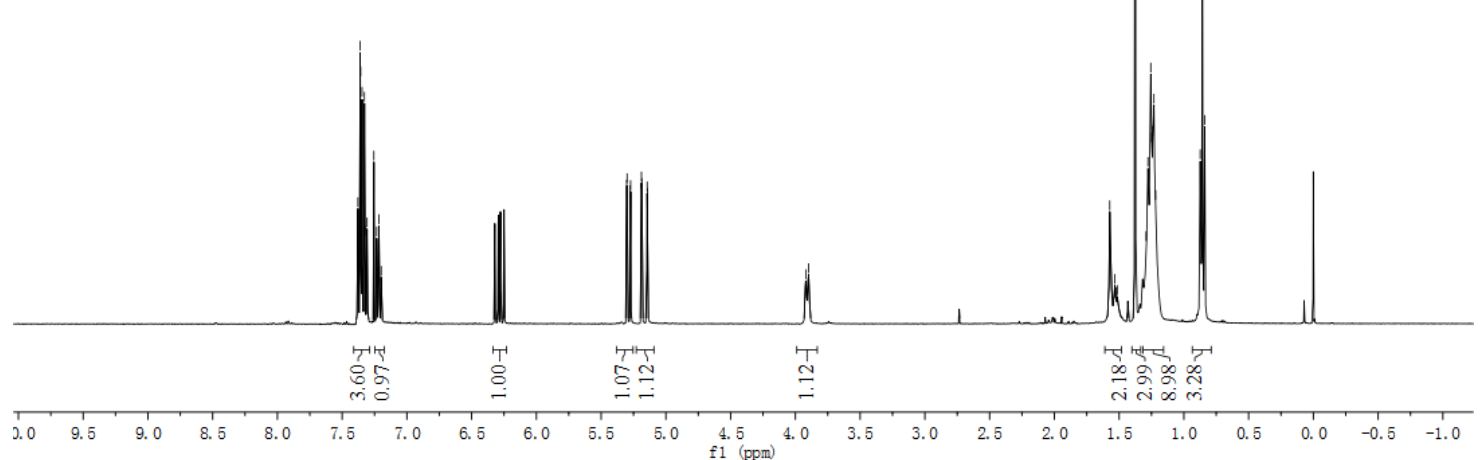


$2 b{ }^{13} \mathrm{C}$<smiles>C=CC(C)(c1ccccc1)C(O)CCCCCC</smiles>
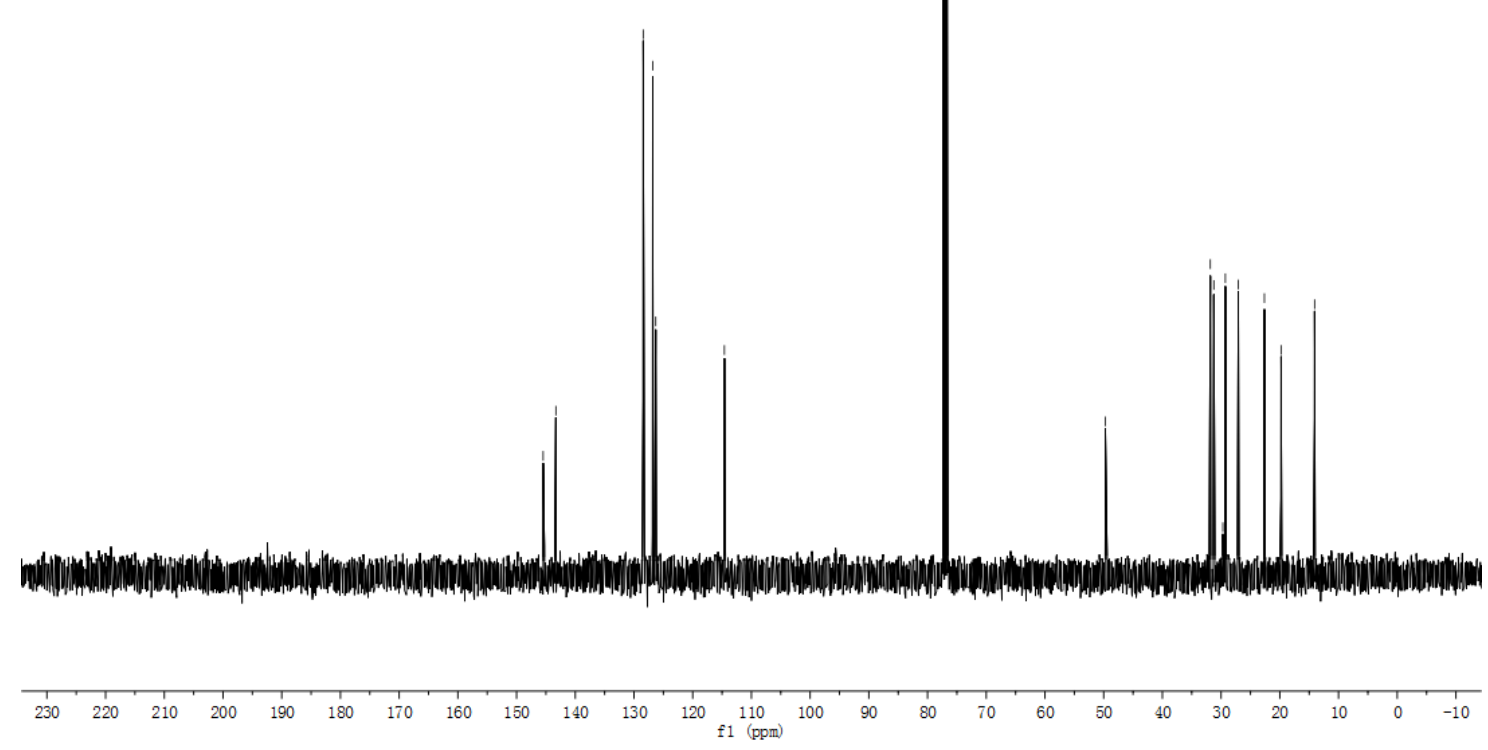

2b HPLC (racemic)

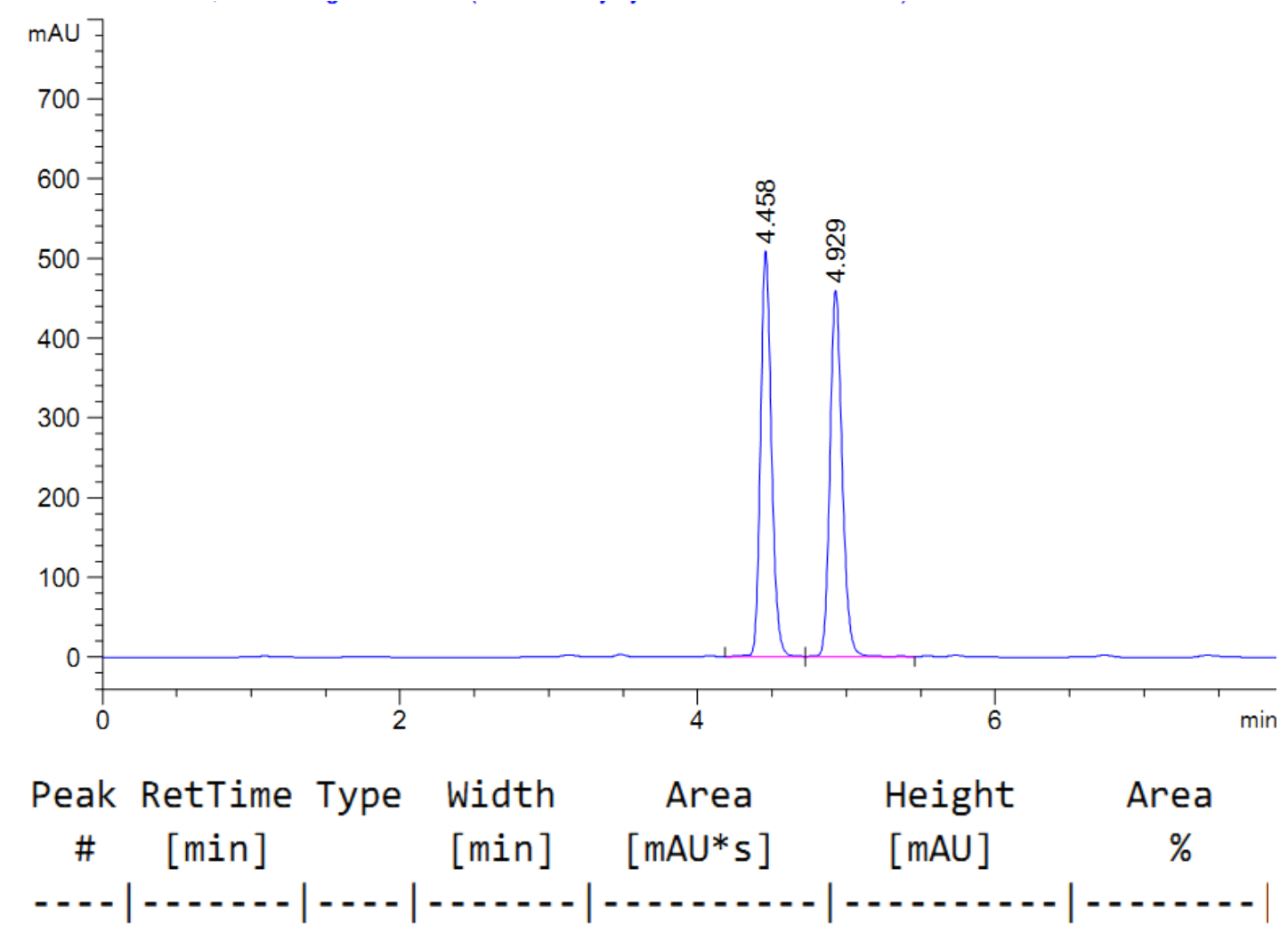

$\begin{array}{llllllll}1 & 4.458 & \text { VB R } & 0.0784 & 2591.83813 & 508.78098 & 49.7862\end{array}$

$\begin{array}{llllllll}2 & 4.929 & B V & R & 0.0874 & 2614.10278 & 459.07004 & 50.2138\end{array}$ 


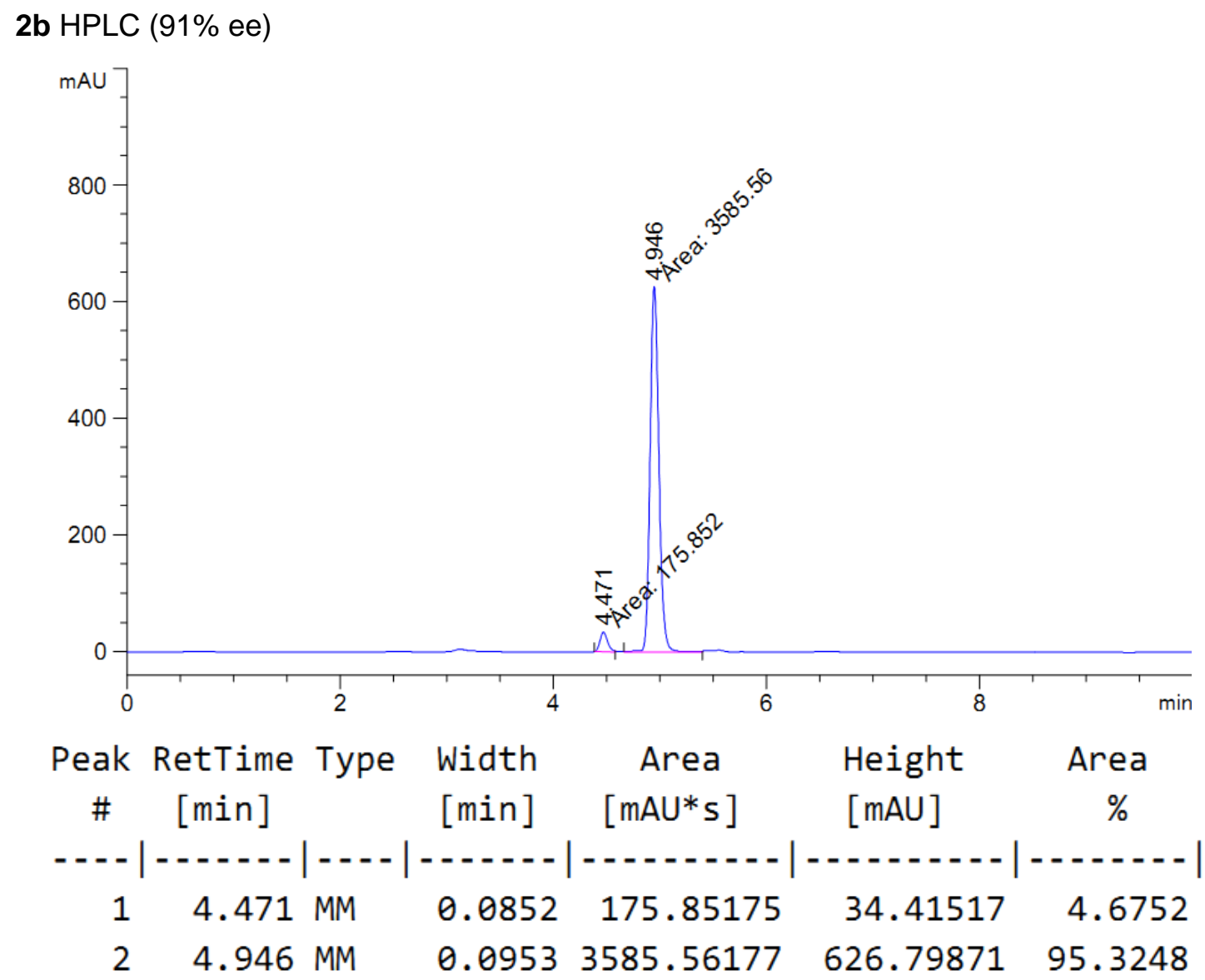

2c ${ }^{1} \mathrm{H}$

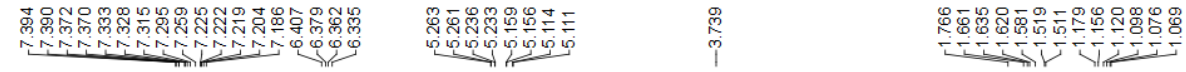

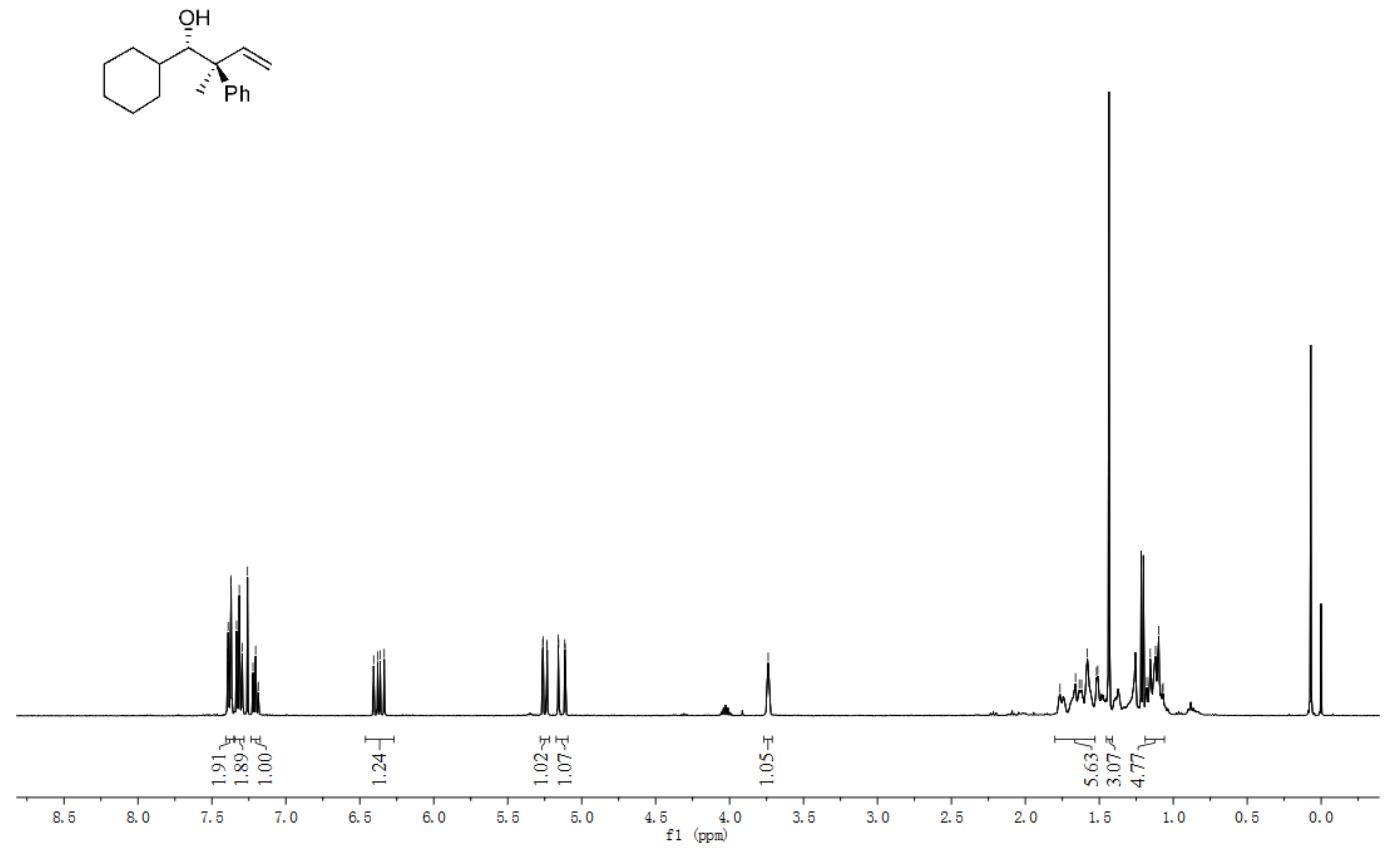


$2 c{ }^{13} \mathrm{C}$<smiles>C=CC(C)(P)C(O)C1CCCCC1</smiles>
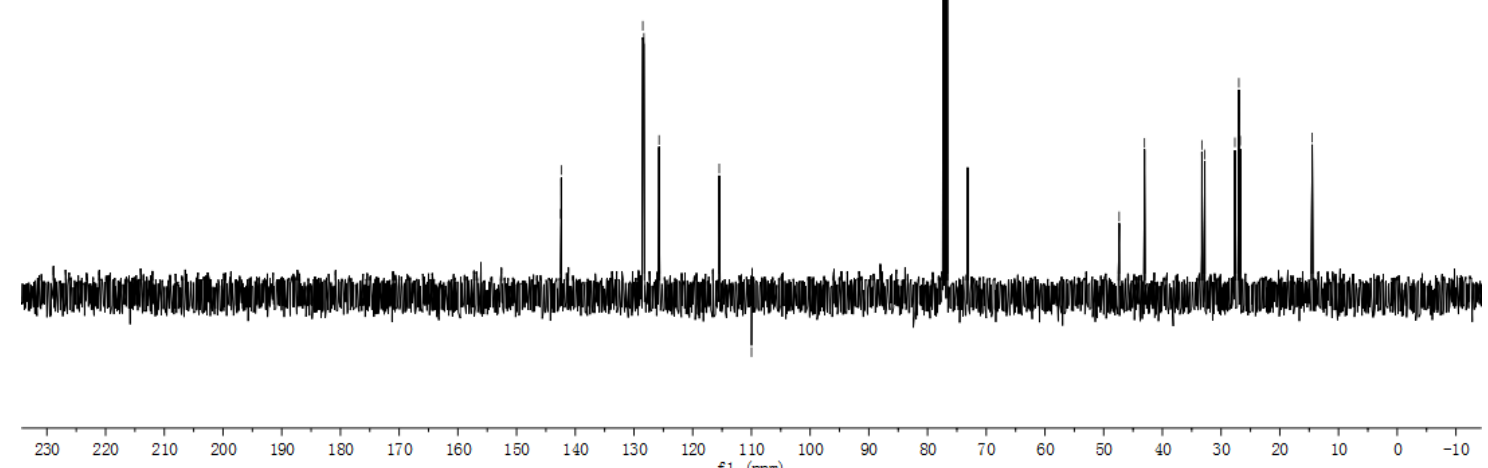

2c HPLC (racemic)

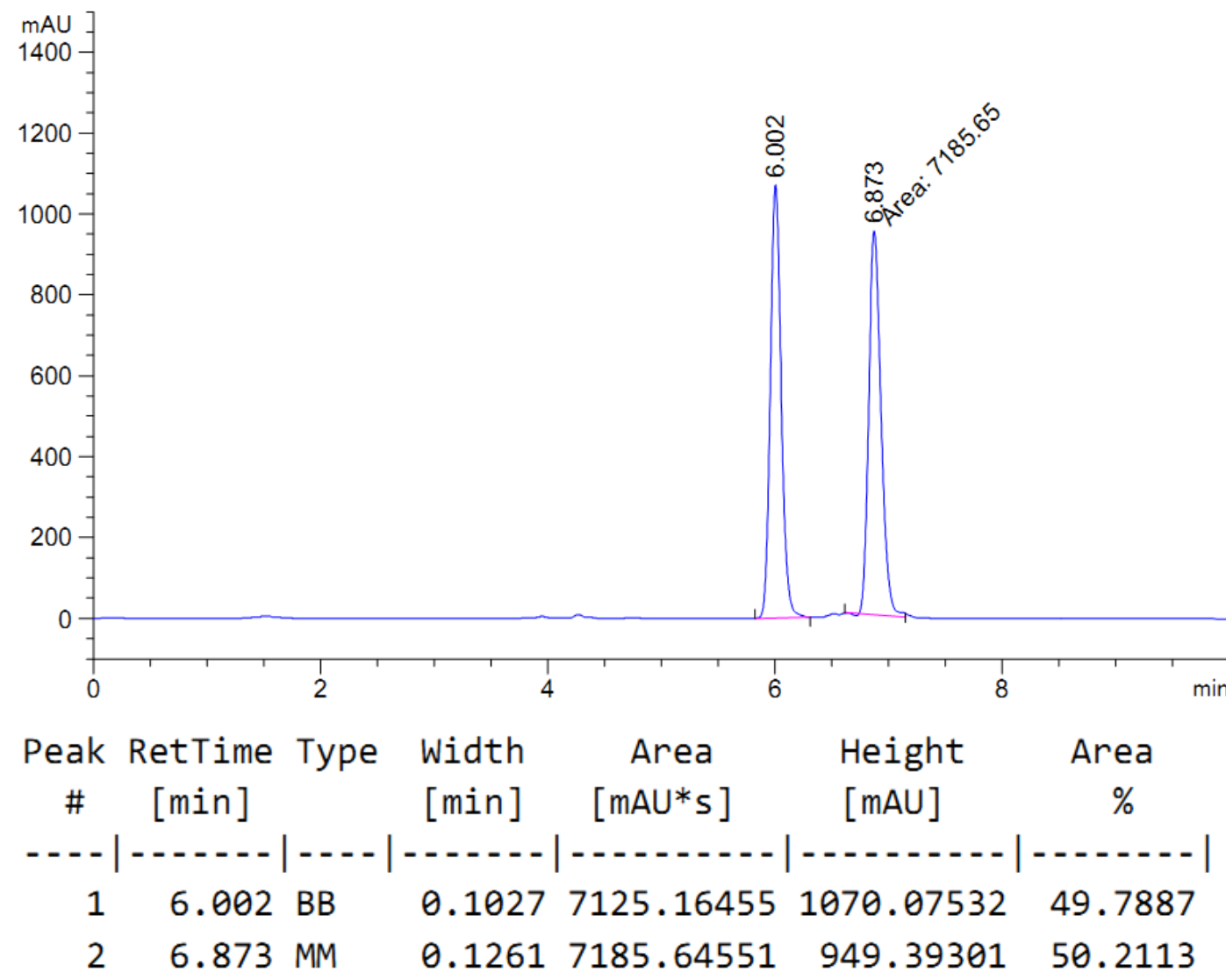


2c HPLC(94 \% ee)

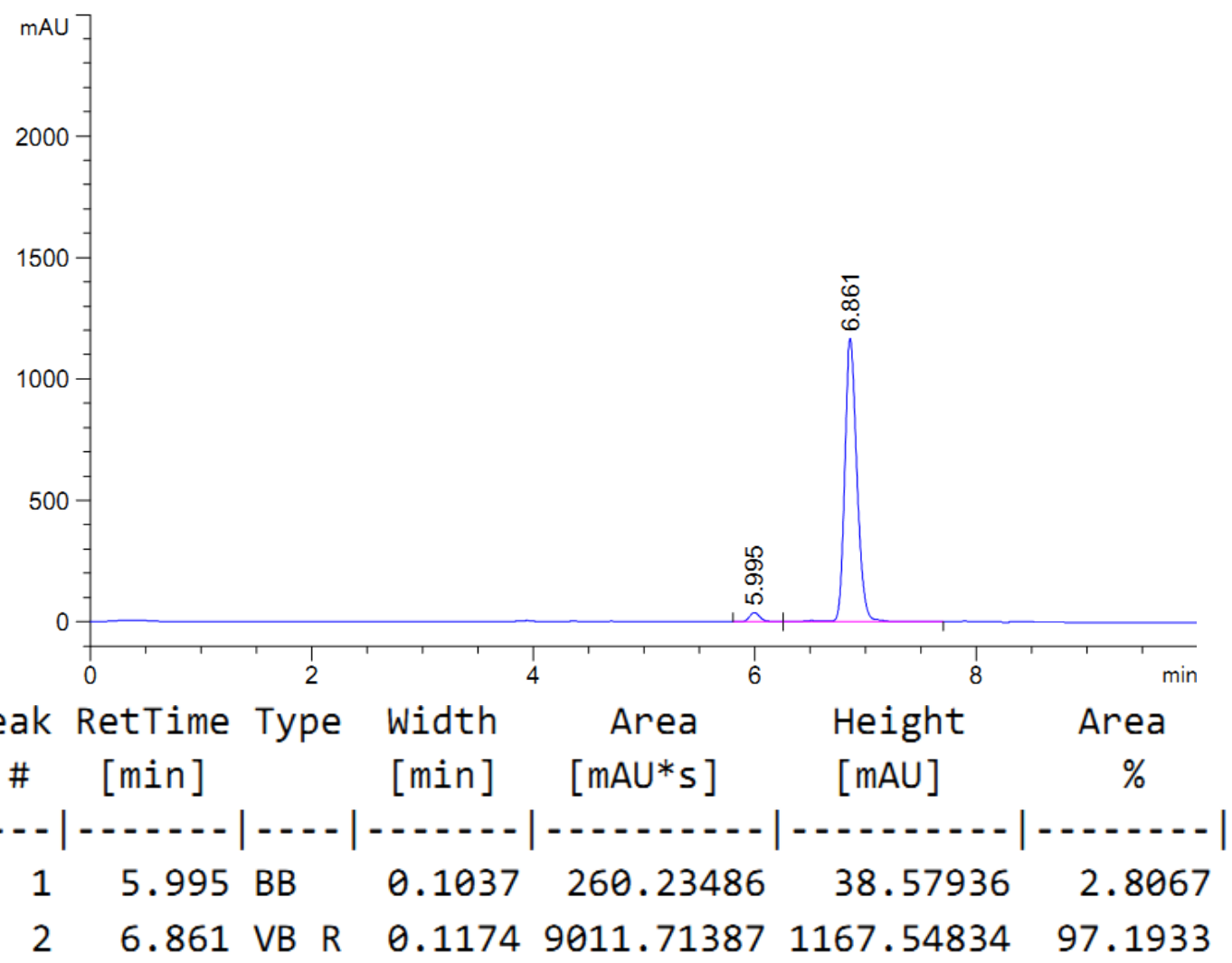

2d ${ }^{1} \mathrm{H}$

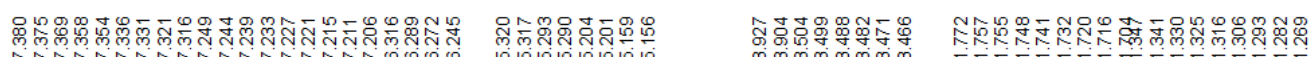<smiles>C=CC(C)(c1ccccc1)C(O)CCCCCl</smiles>

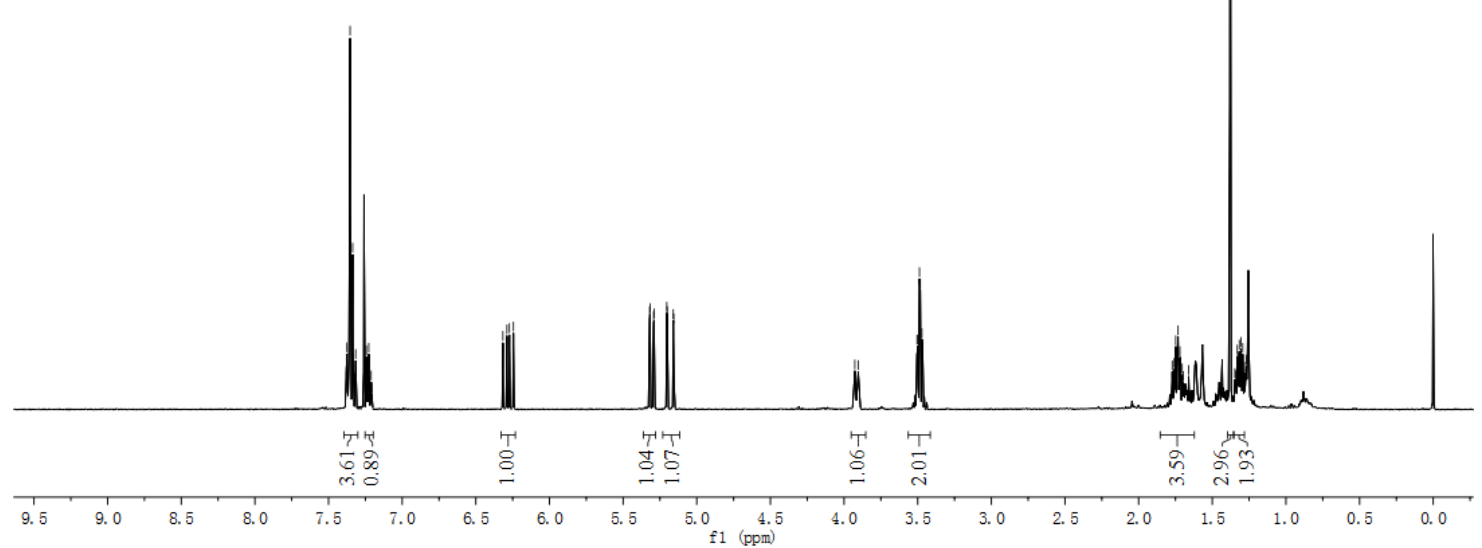


$2 \mathbf{d}^{13} \mathrm{C}$

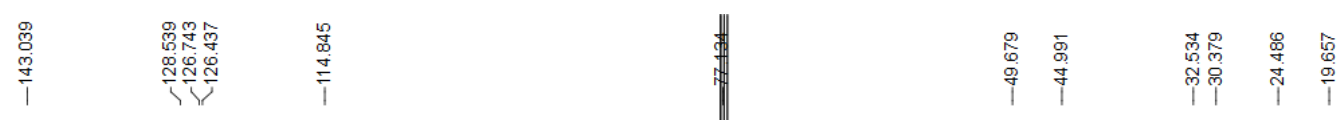<smiles>CC(C)(C)C(O)CCCCCl</smiles>

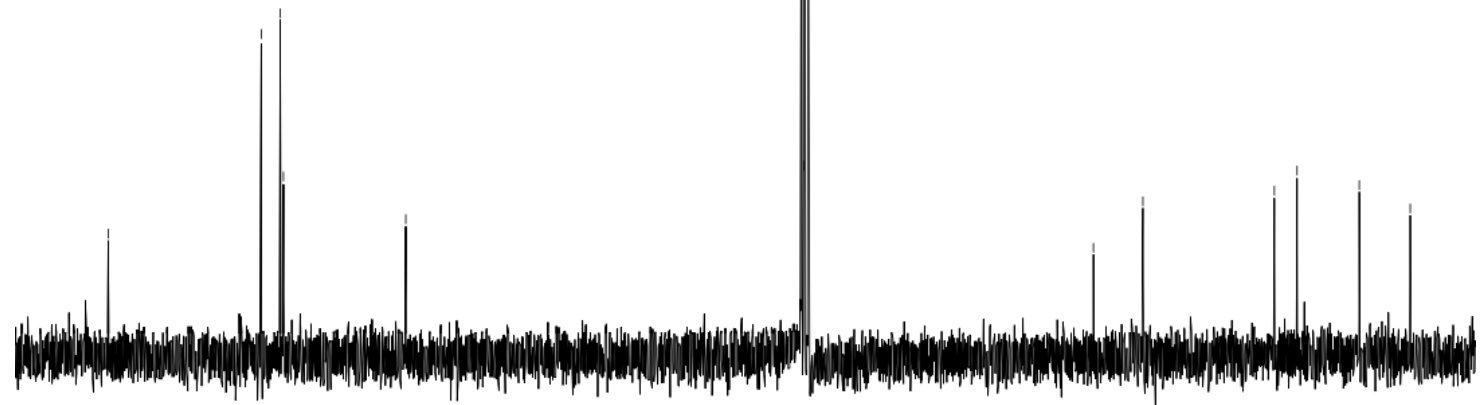

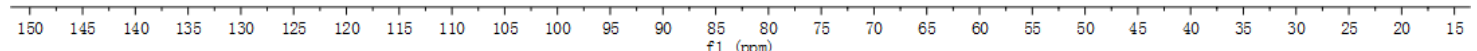

2d HPLC (racemic)

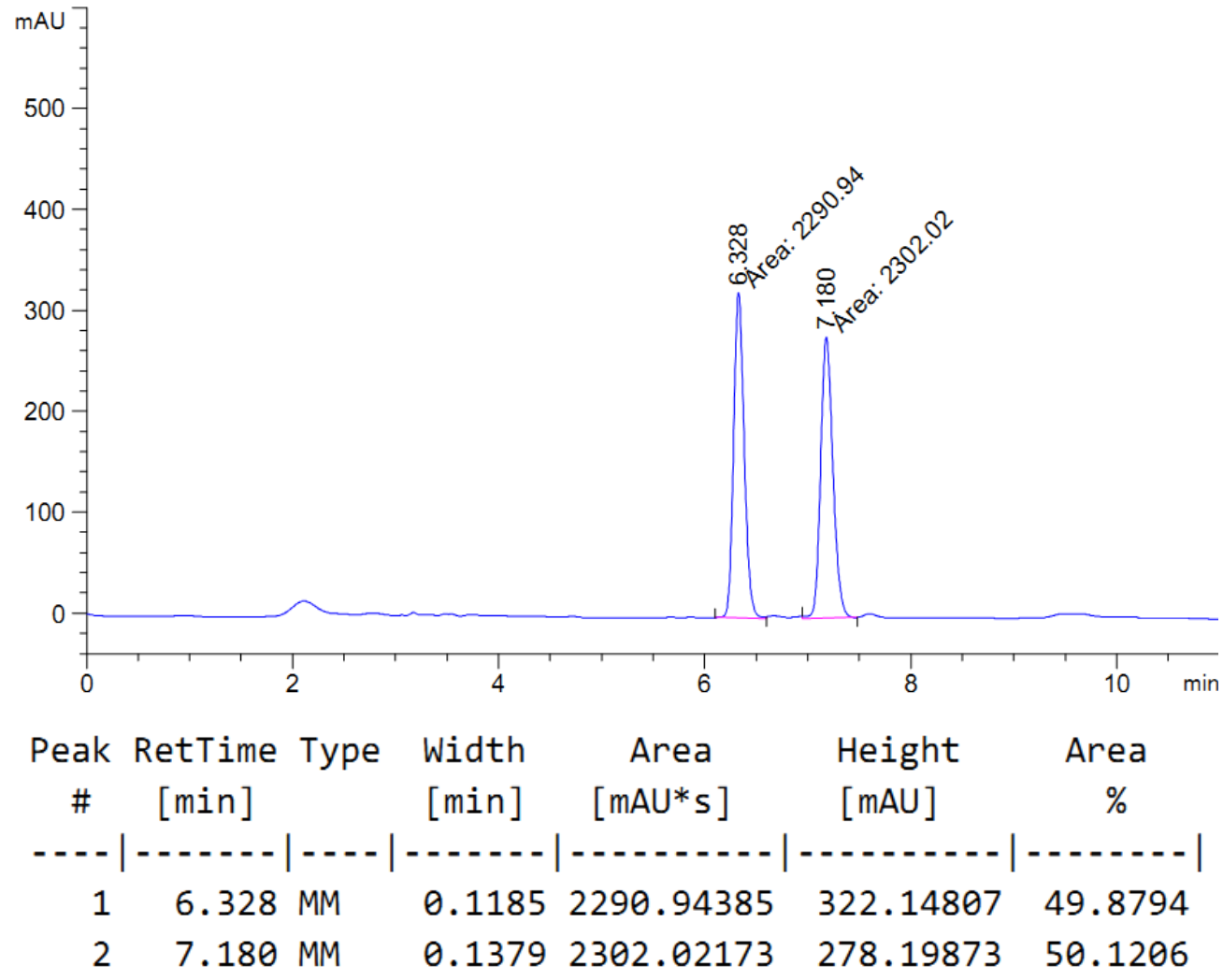




\section{2d HPLC(90\% ee)}

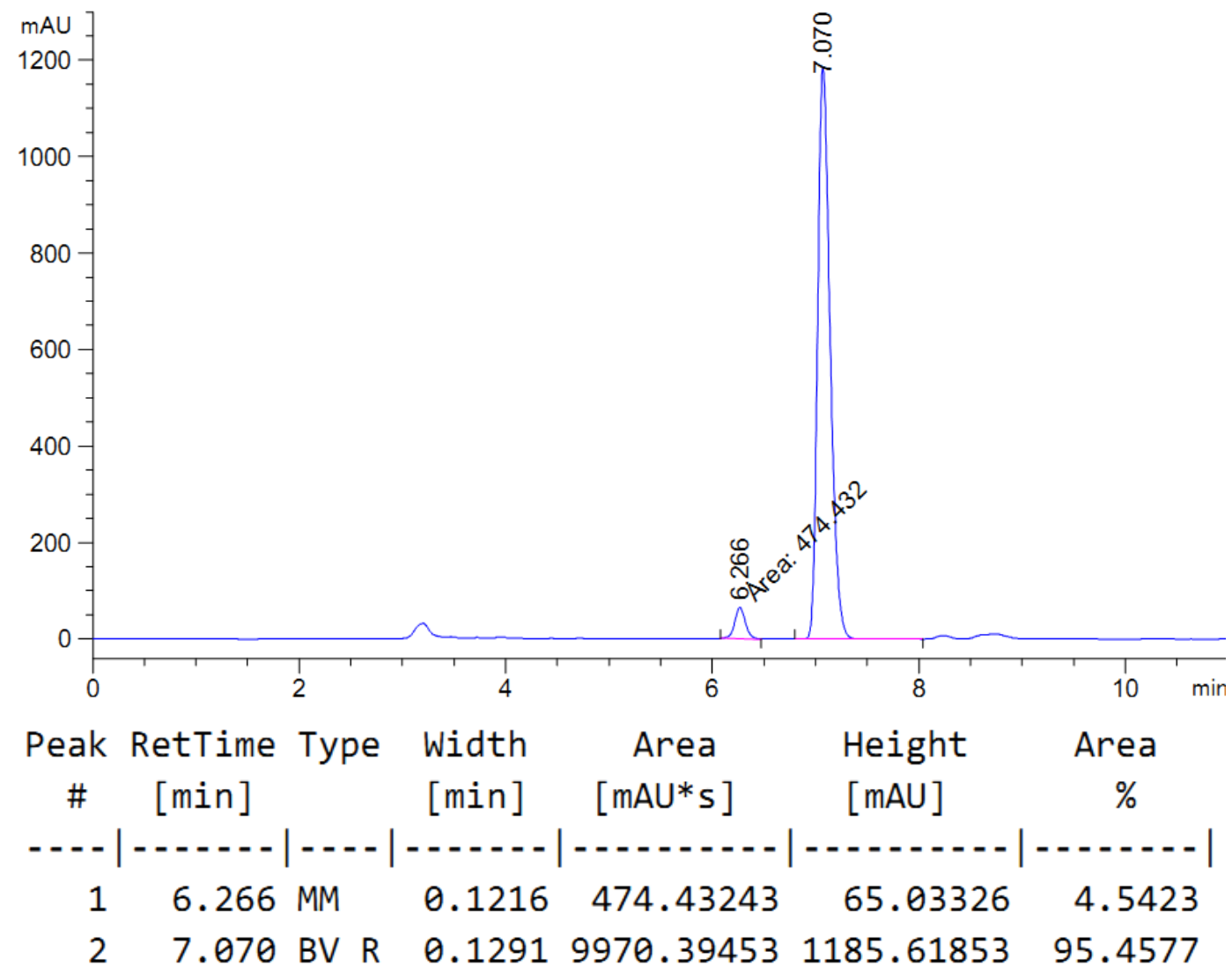

$2 e^{1} \mathrm{H}$

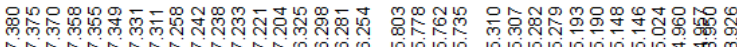

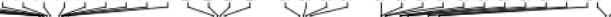<smiles>C=CCCC(O)C(C)(C=C)c1ccccc1</smiles>

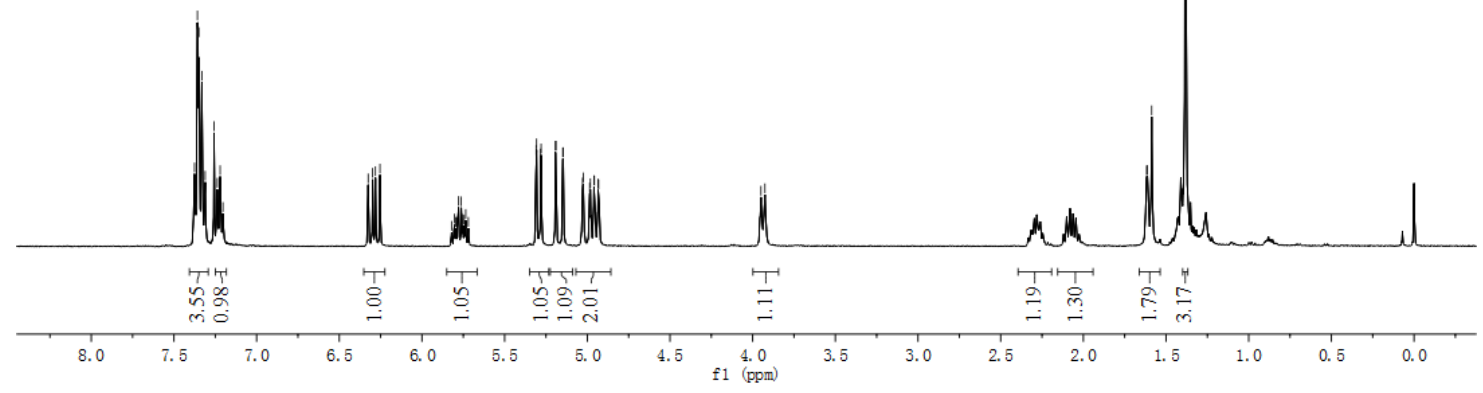


$2 e^{13} \mathrm{C}$
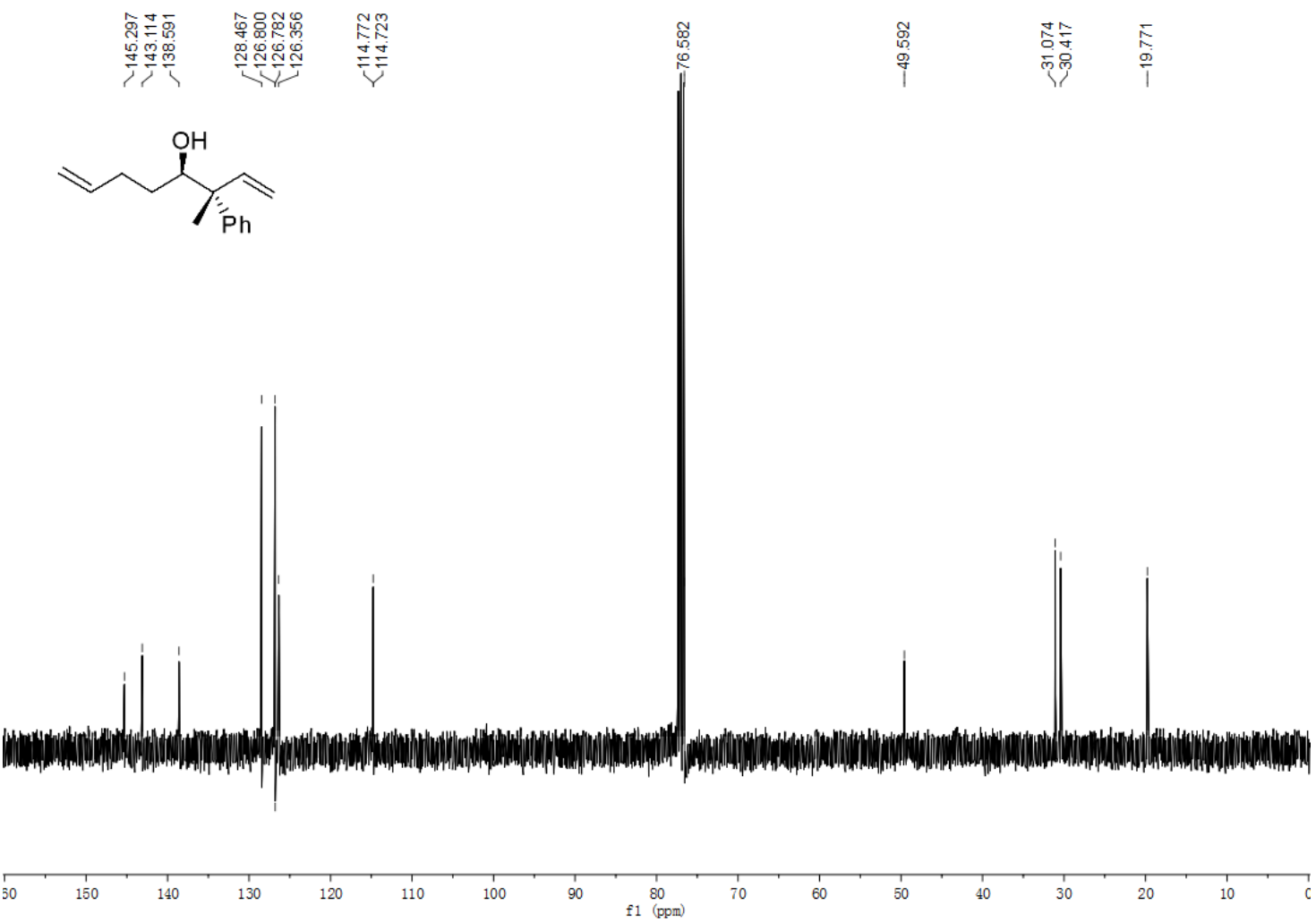

2e HPLC (racemic)

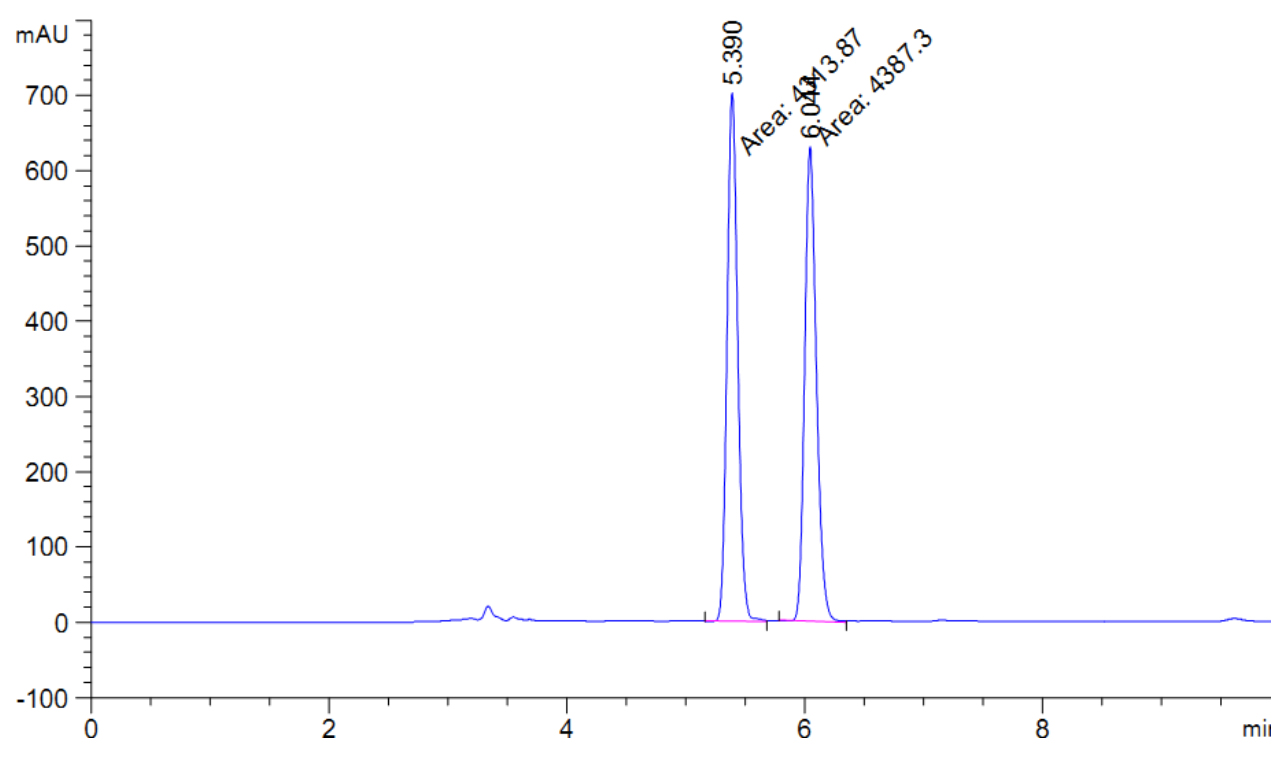

Peak RetTime Type Width Area Height Area

\# [min] [min] [mAU*s] [mAU]

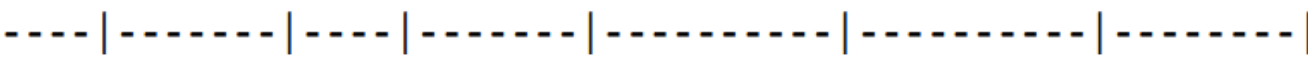

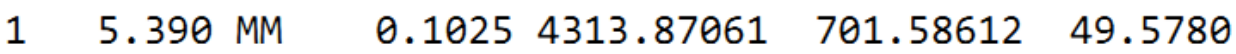

$\begin{array}{lllllll}2 & 6.044 \text { MM } & 0.1160 & 4387.30371 & 630.21417 & 50.4220\end{array}$ 
2e HPLC(90\% ee)
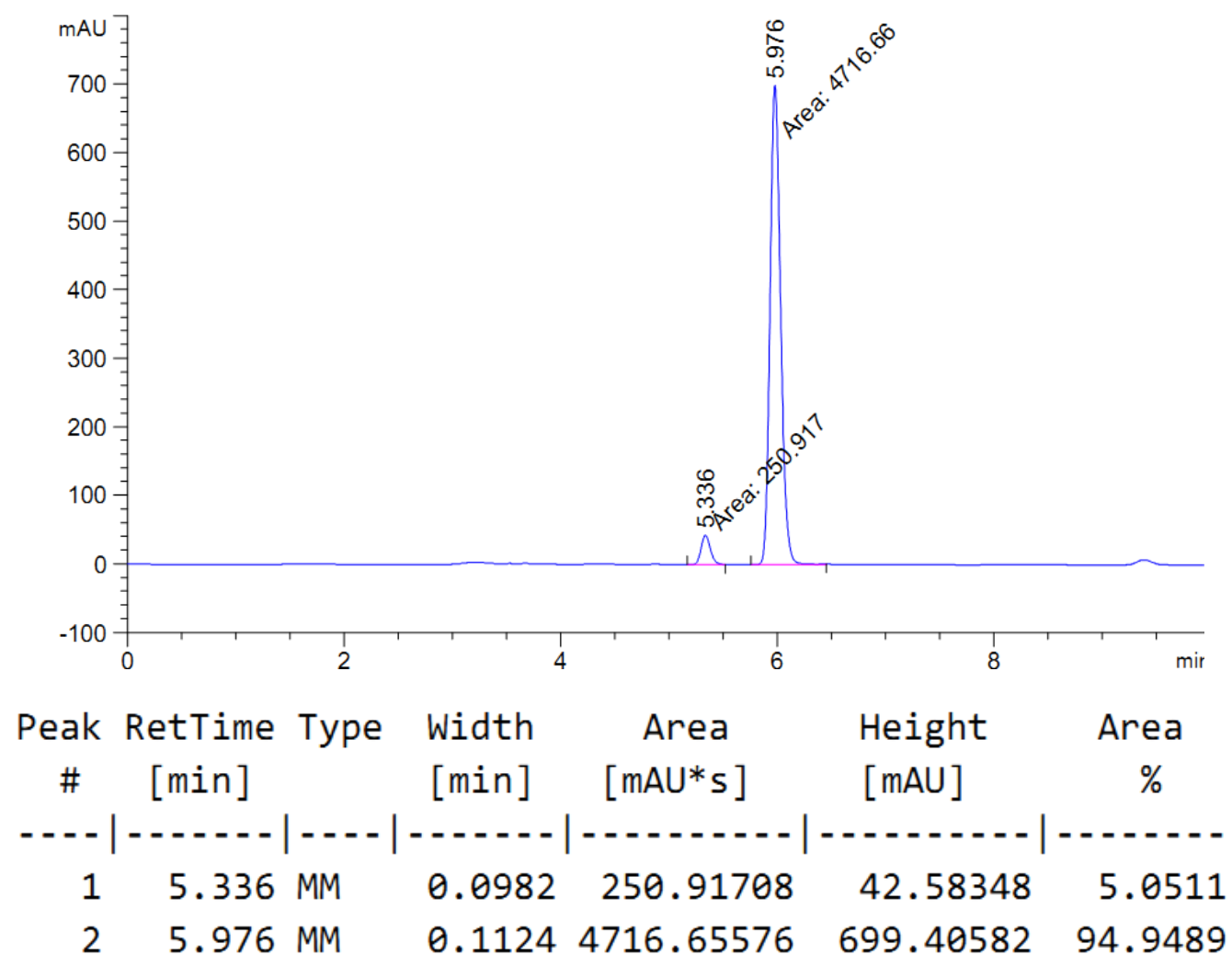

$2 f^{1} \mathrm{H}$

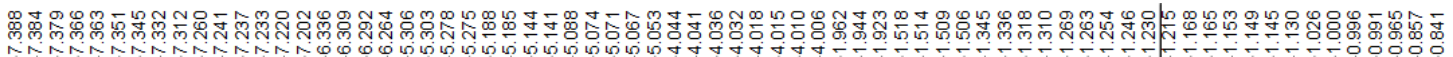<smiles>CC=CC(C)(c1ccccc1C)C(O)CCCCC=C(C)C</smiles>

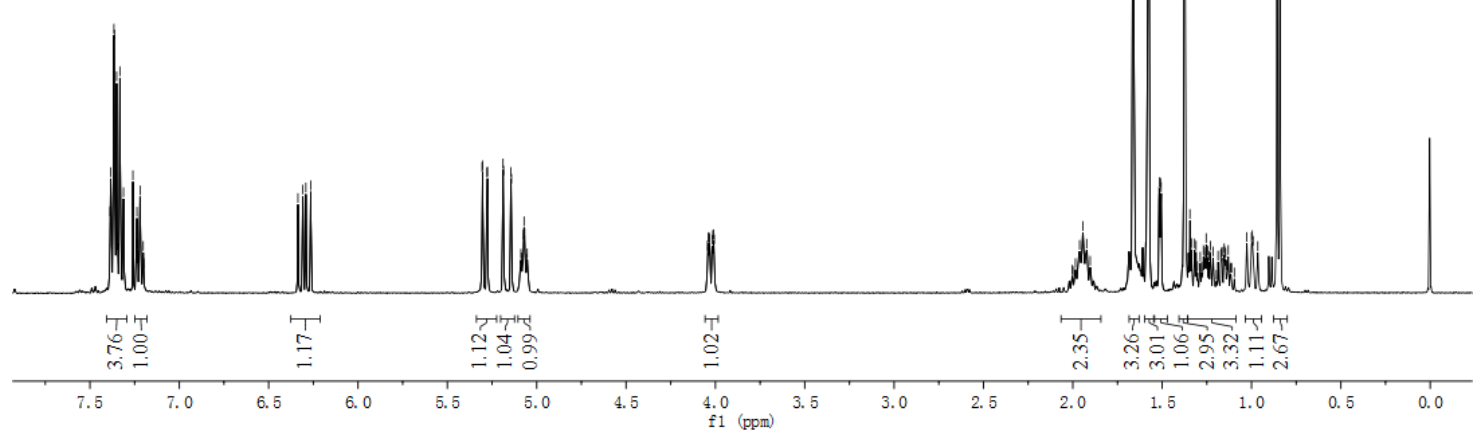


$2 f^{13} \mathrm{C}$
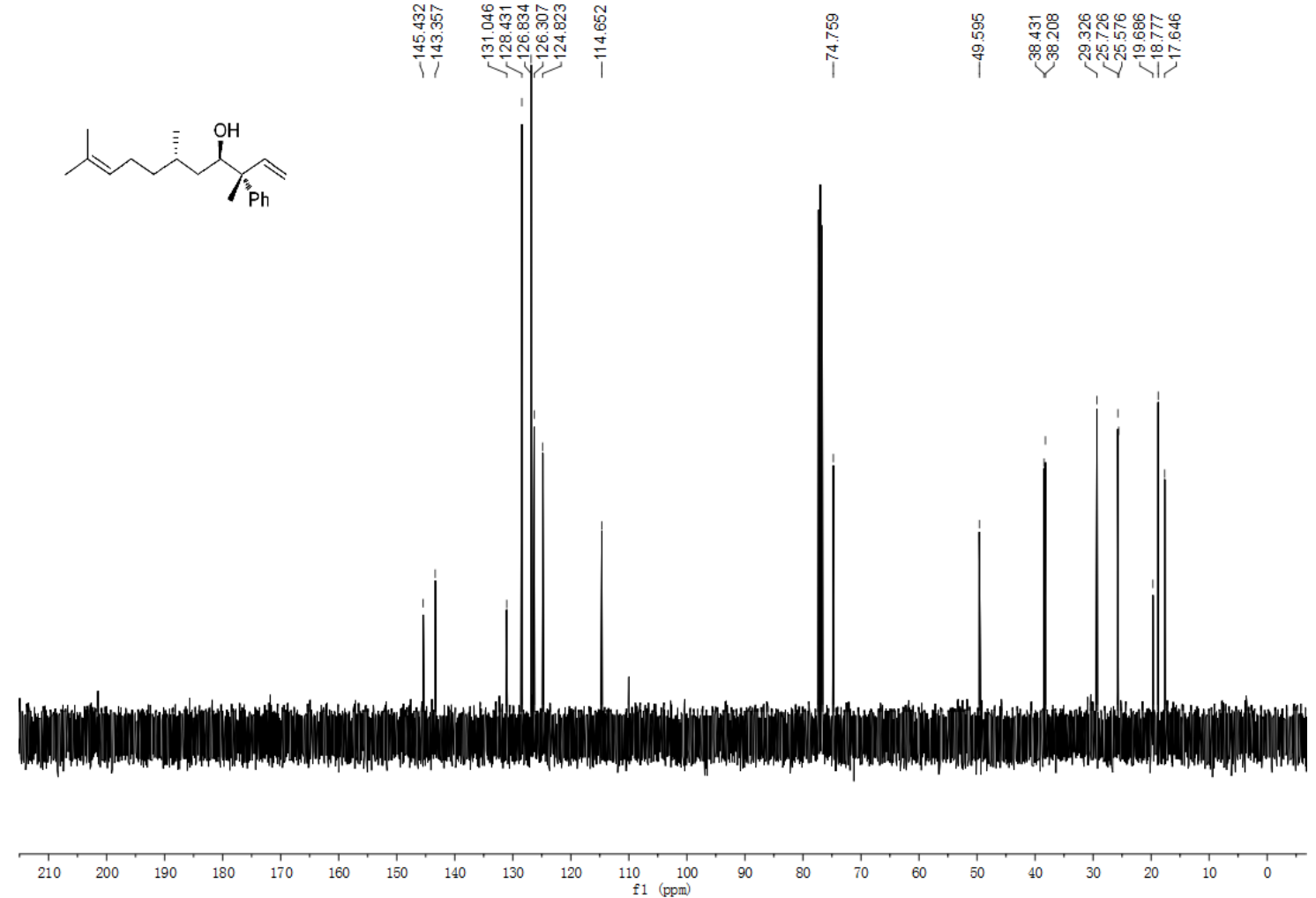

2f HPLC (racemic)

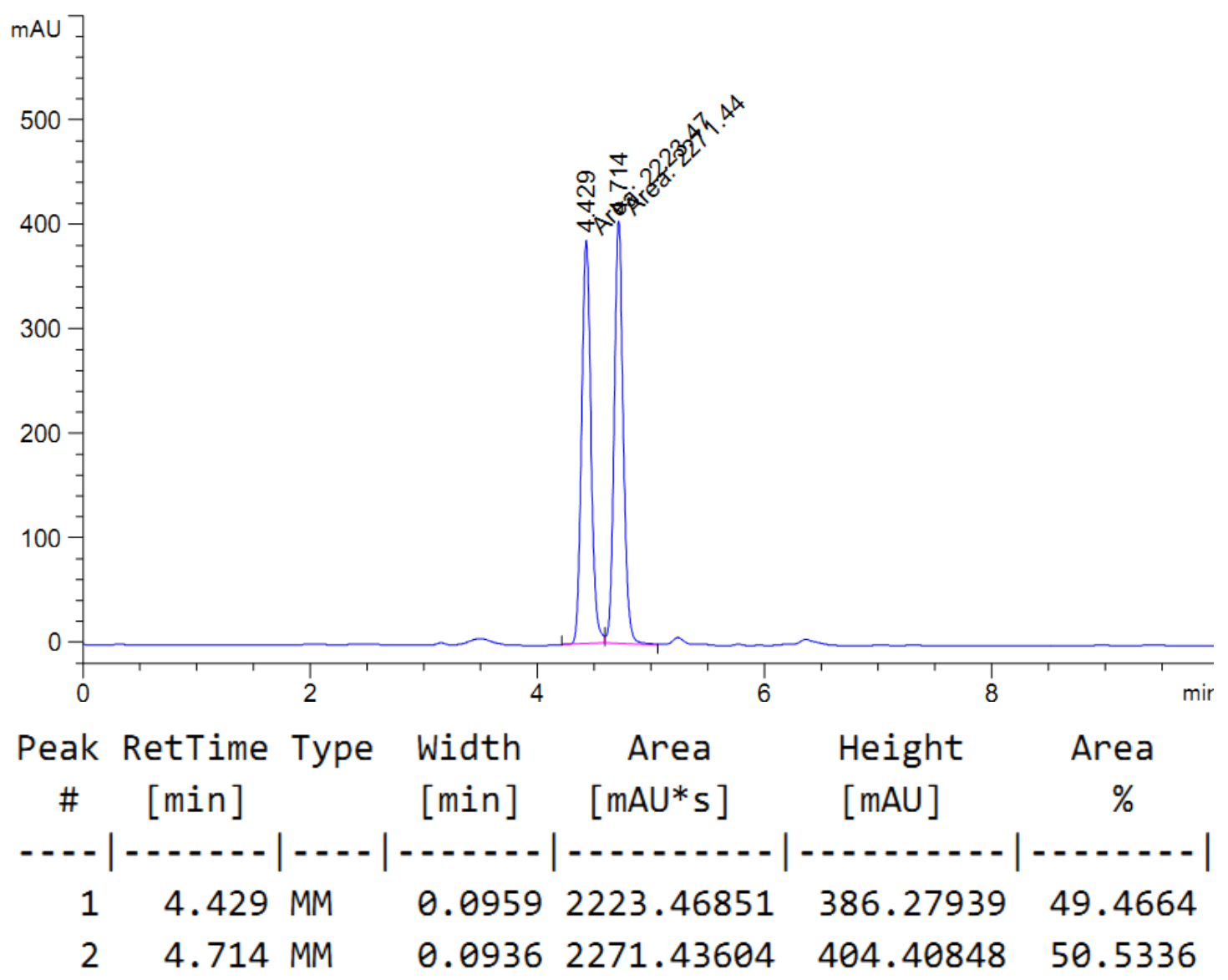


2f $\operatorname{HPLC}(92 \%$ ee $)$

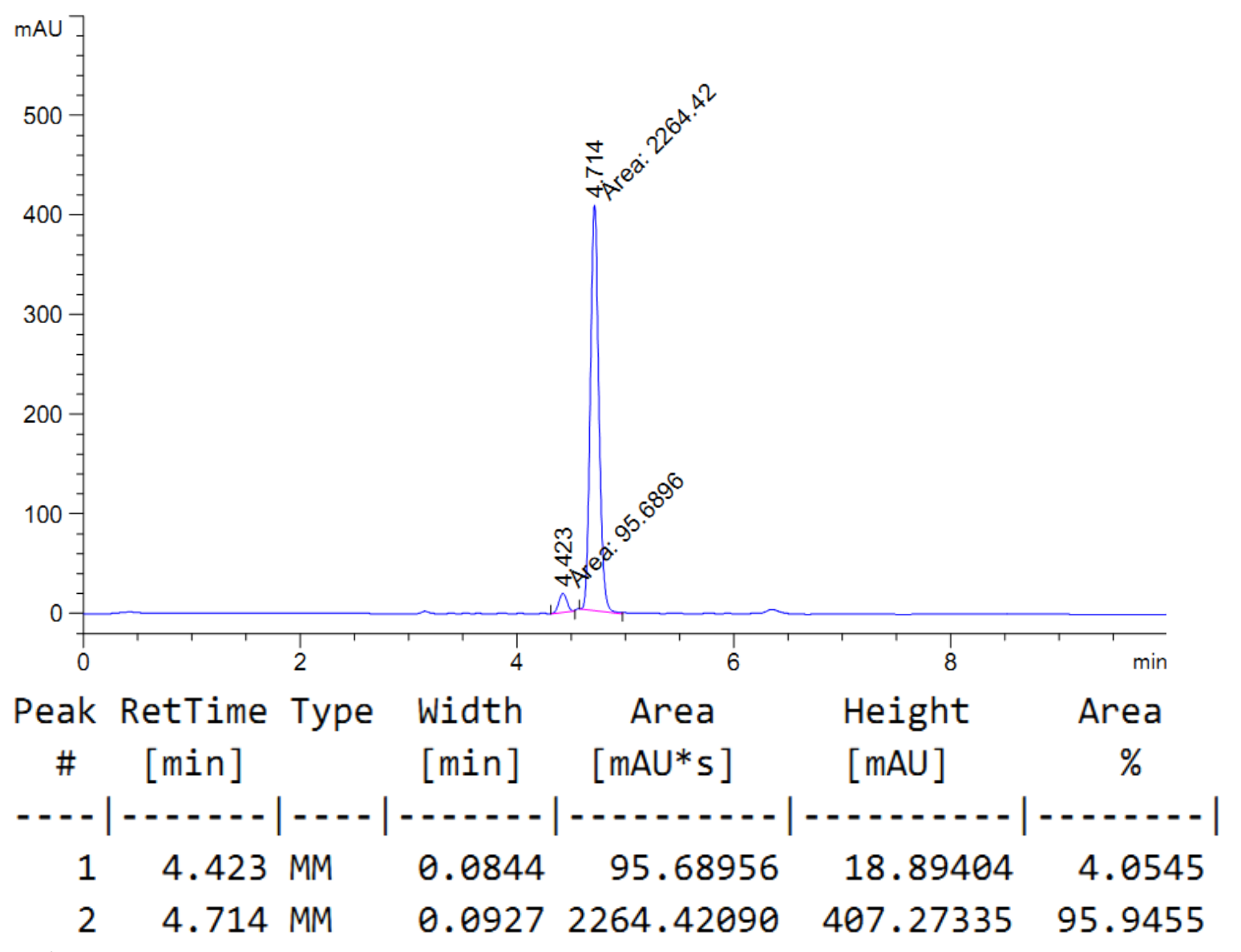

$2 g^{1} \mathrm{H}$<smiles>CCC(C)(c1ccccc1)C(O)CCc1ccccc1</smiles>

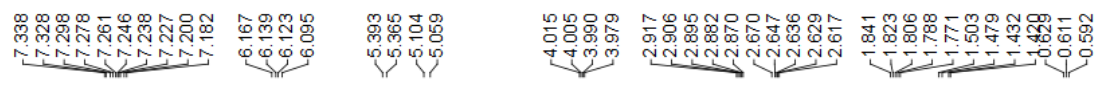

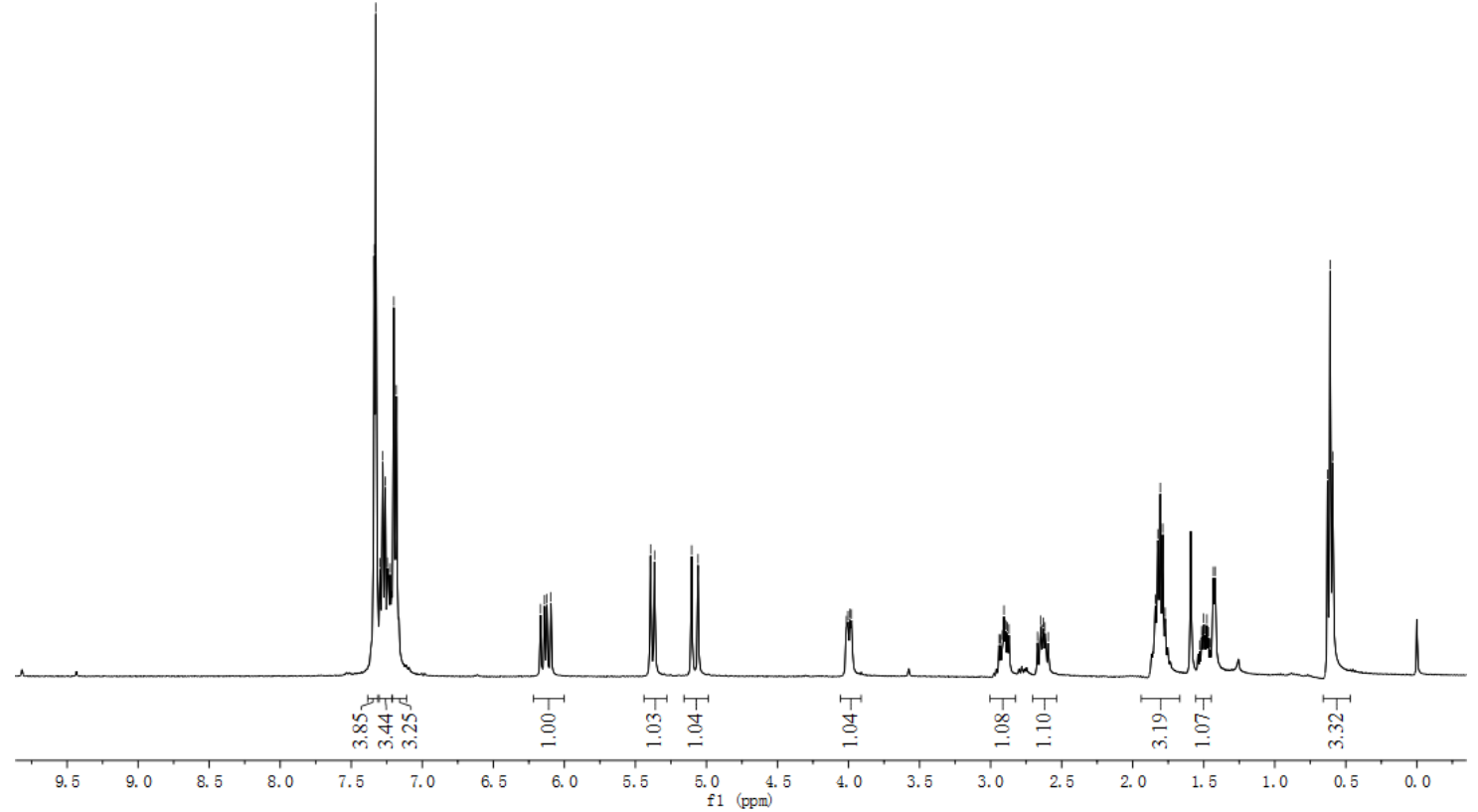



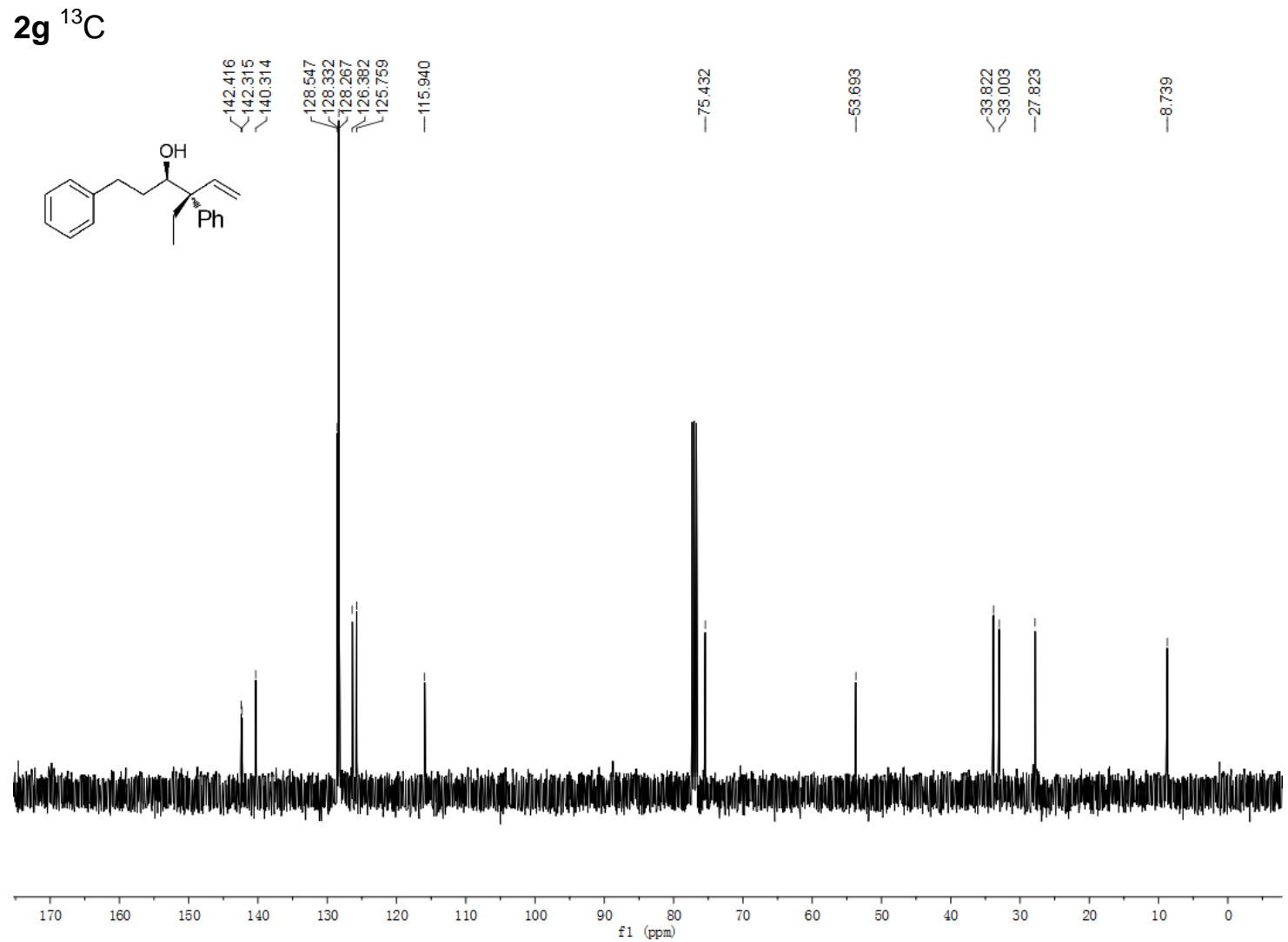

2g HPLC (racemic)

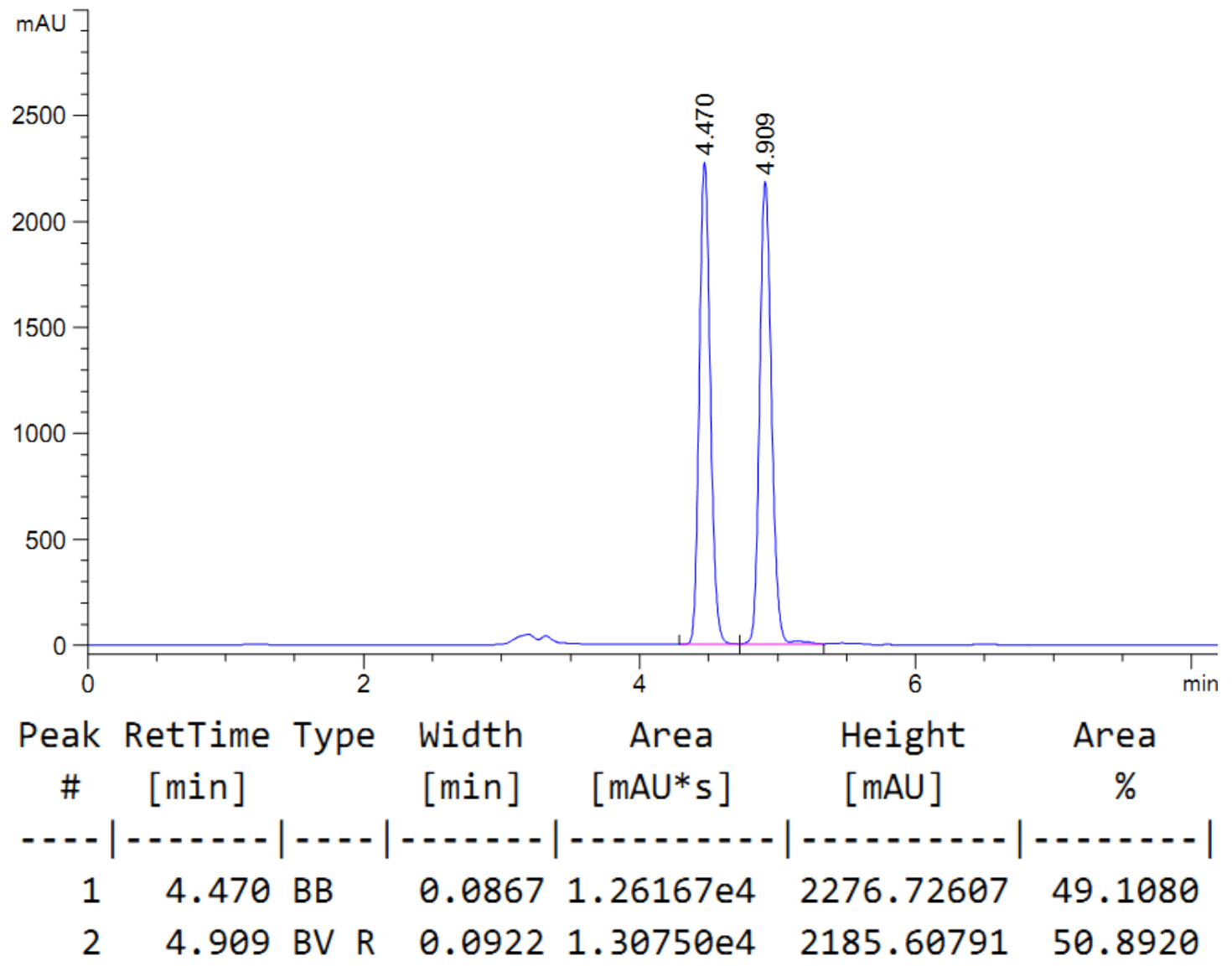




\section{2g HPLC(92\% ee)}

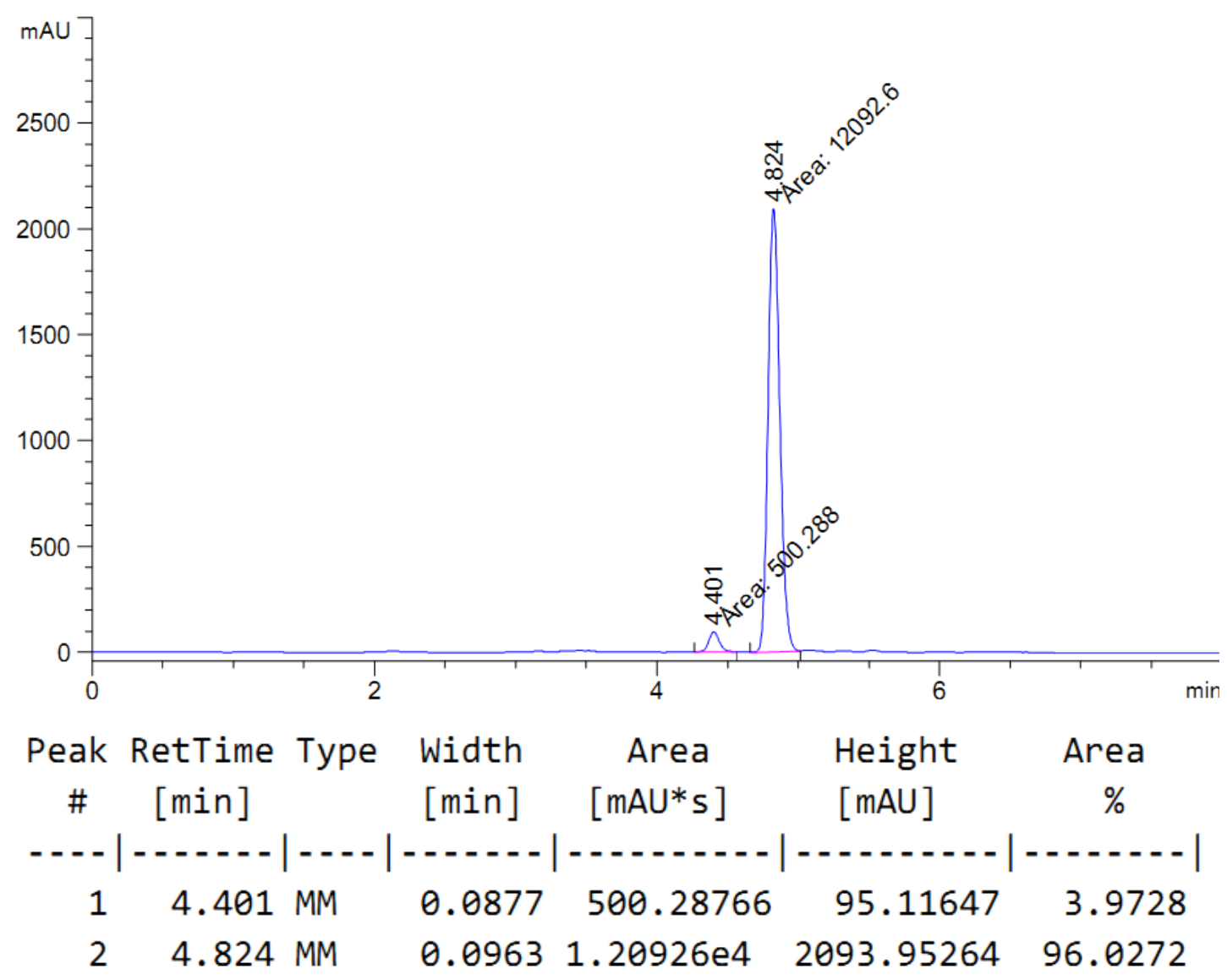

2h ${ }^{1} \mathrm{H}$

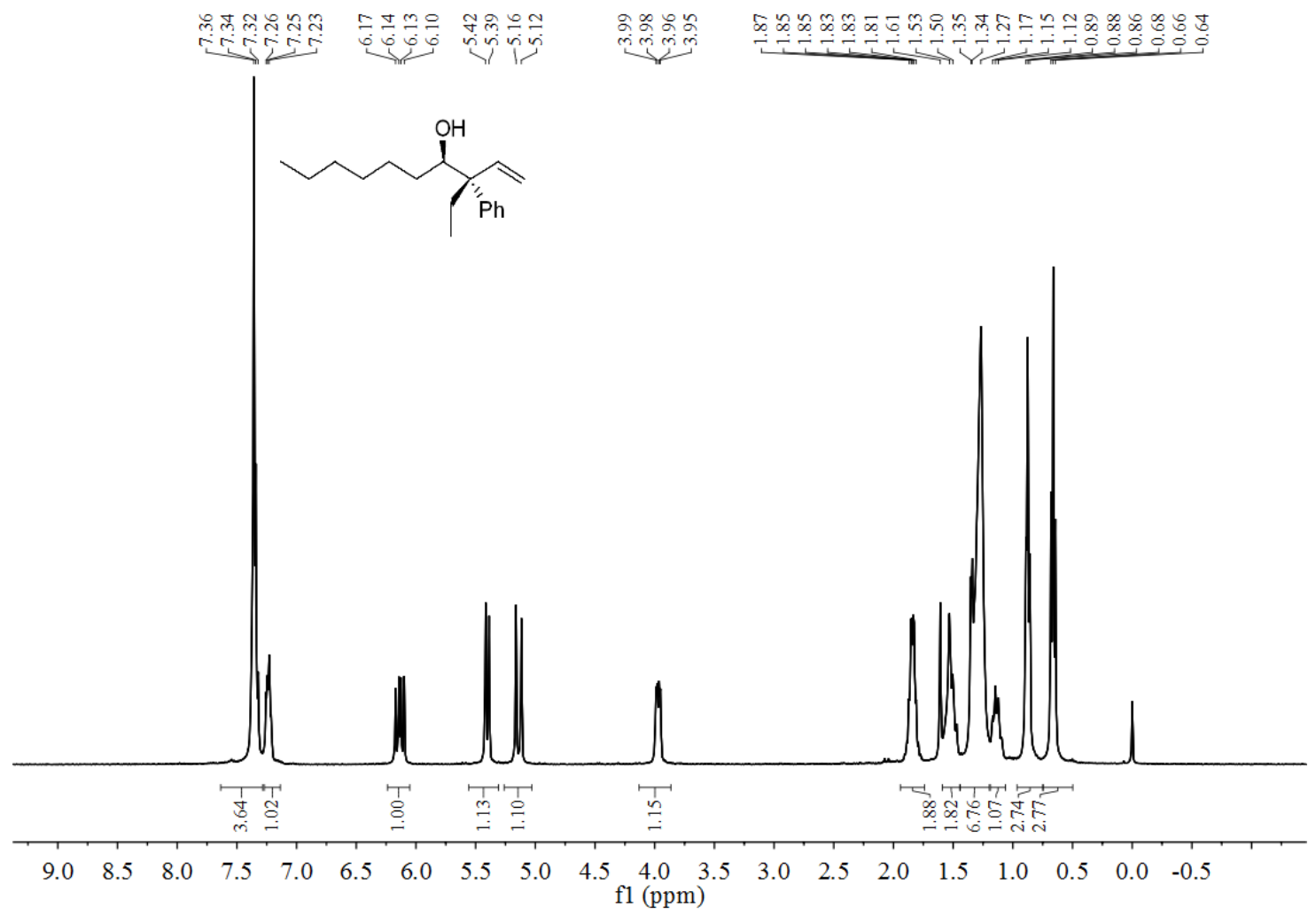


$2 h{ }^{13} \mathrm{C}$
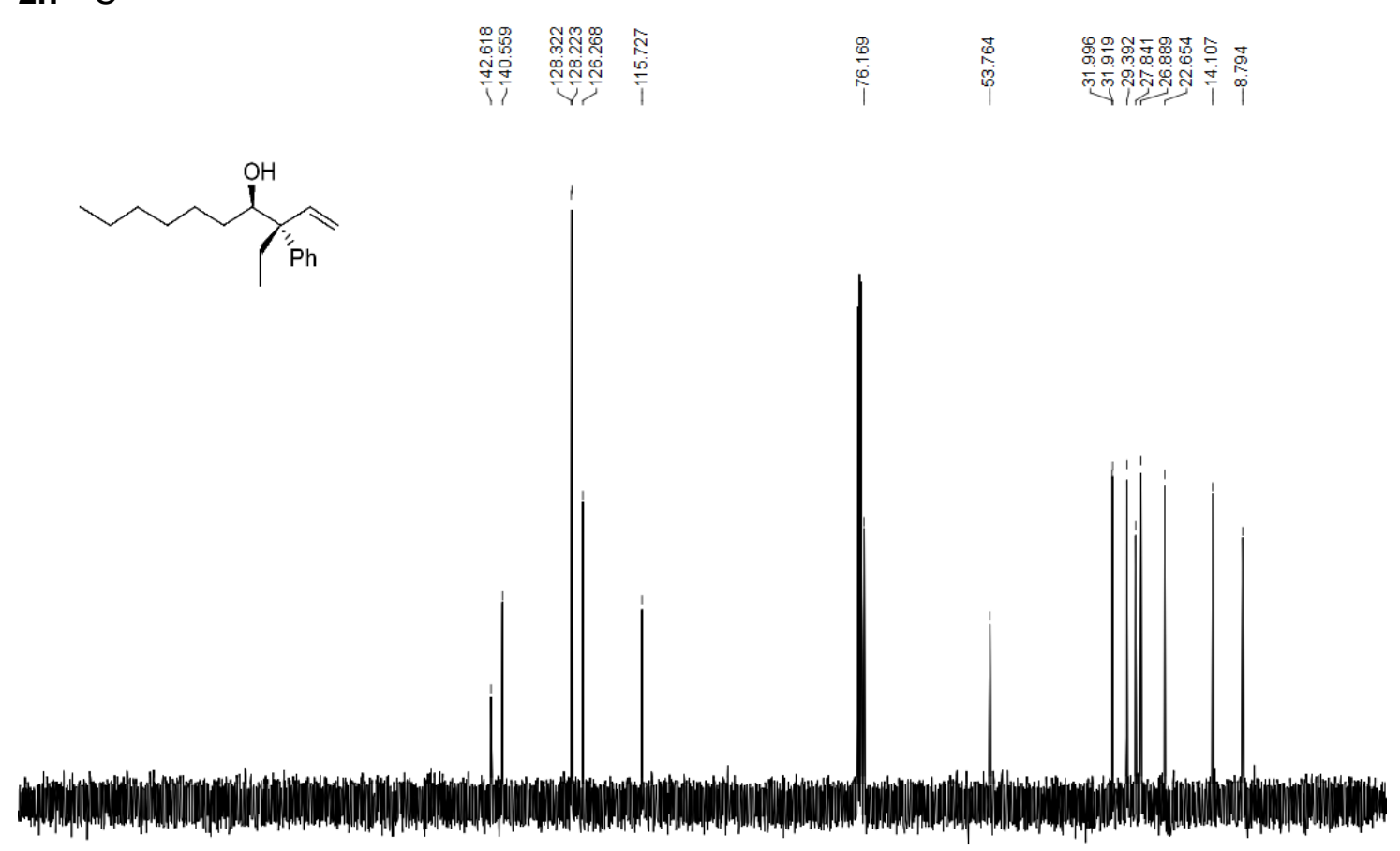

$\begin{array}{lllllllllllll}220 & 210 & 200 & 190 & 180 & 170 & 160 & 150 & 140 & 130 & 120 & 110 & 100 \\ f 1 & (\mathrm{ppm})\end{array}$

2h HPLC (racemic)

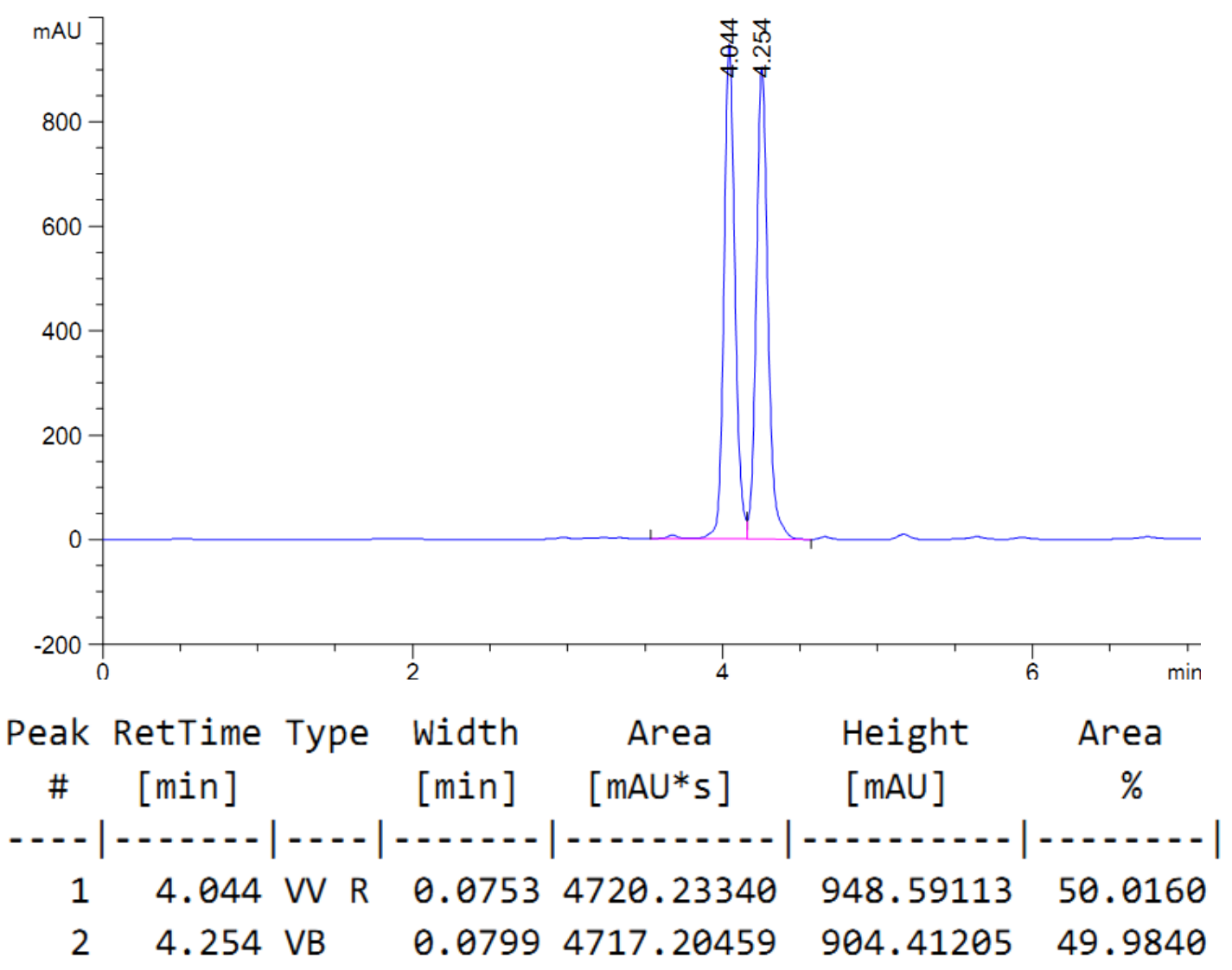




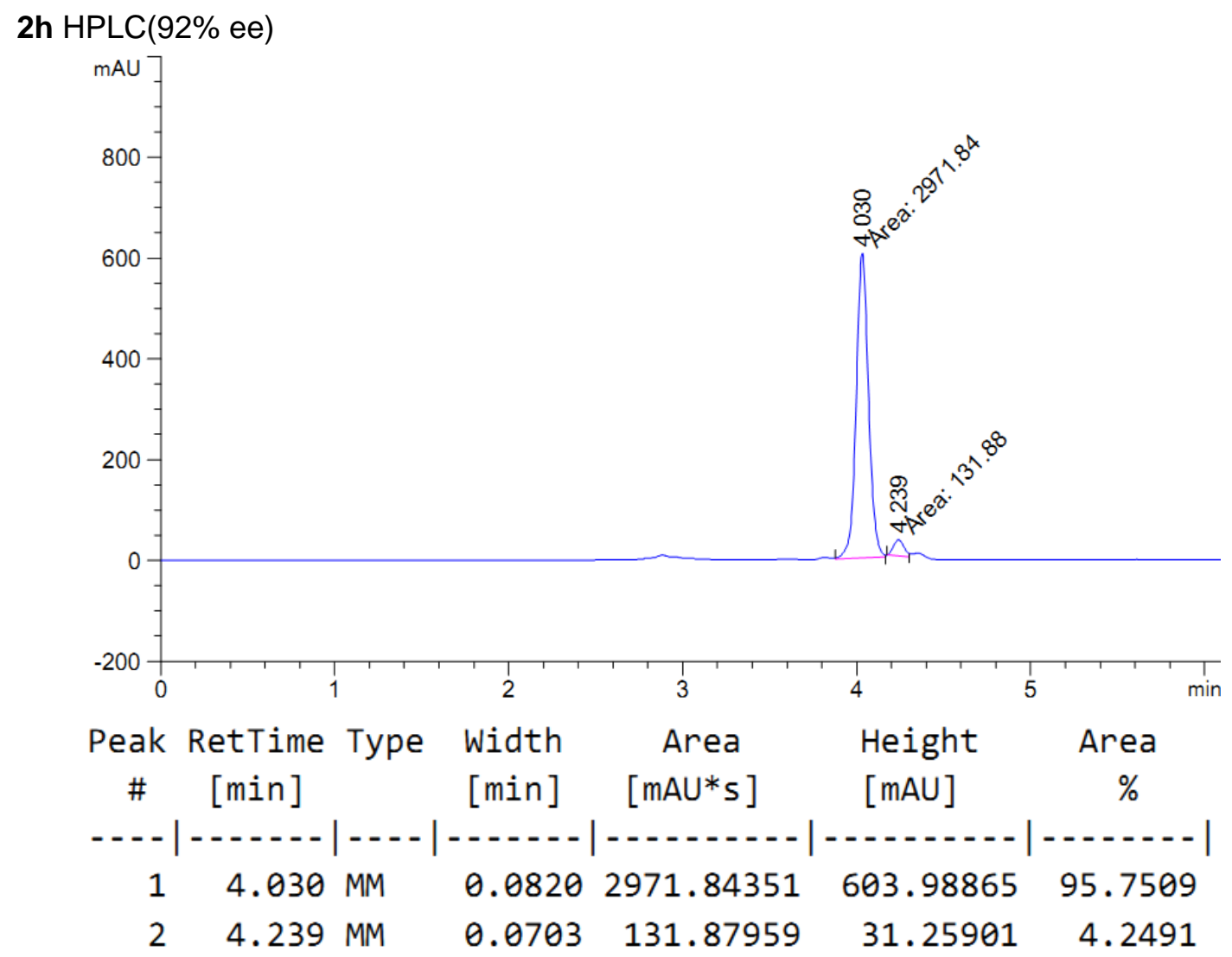

$2 \mathbf{i}^{1} \mathrm{H}$

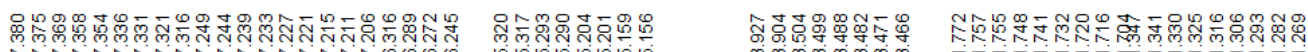
$\mathrm{Cl}_{\mathrm{P} \text { Ph }}^{\mathrm{OH}}$

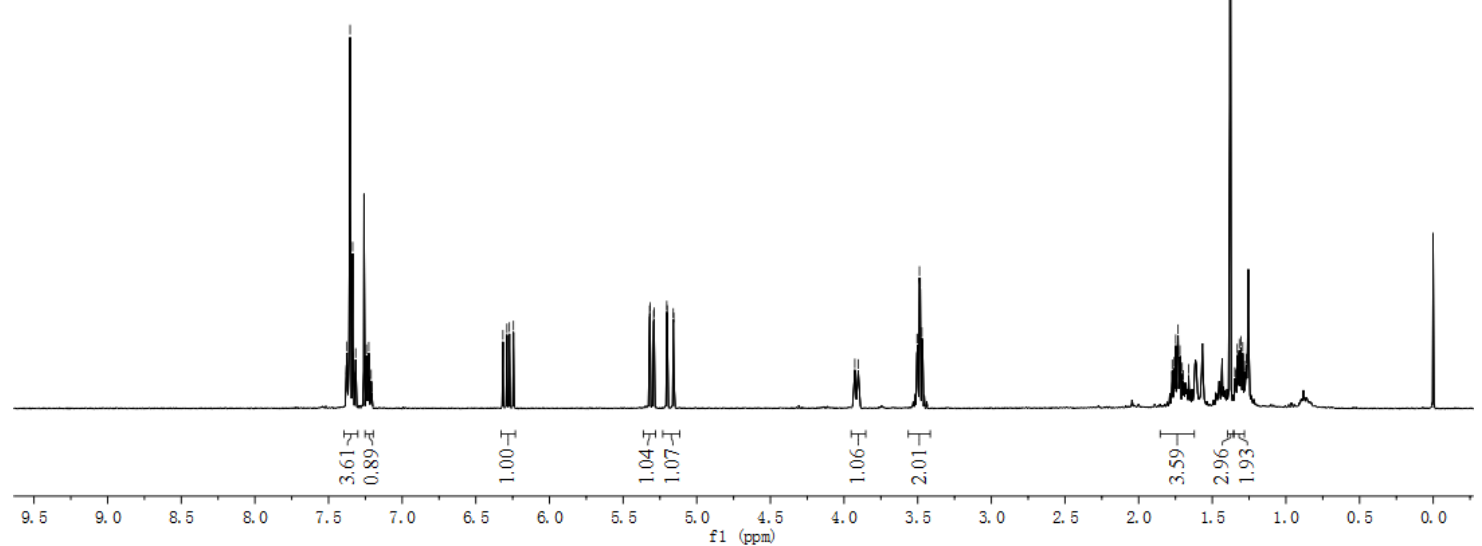


$2 \mathbf{i}^{13} \mathrm{C}$

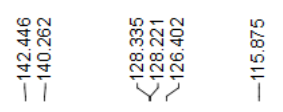

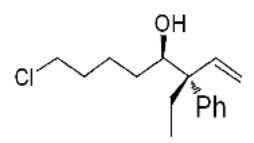

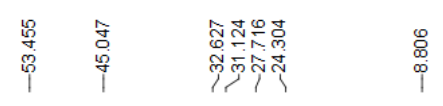
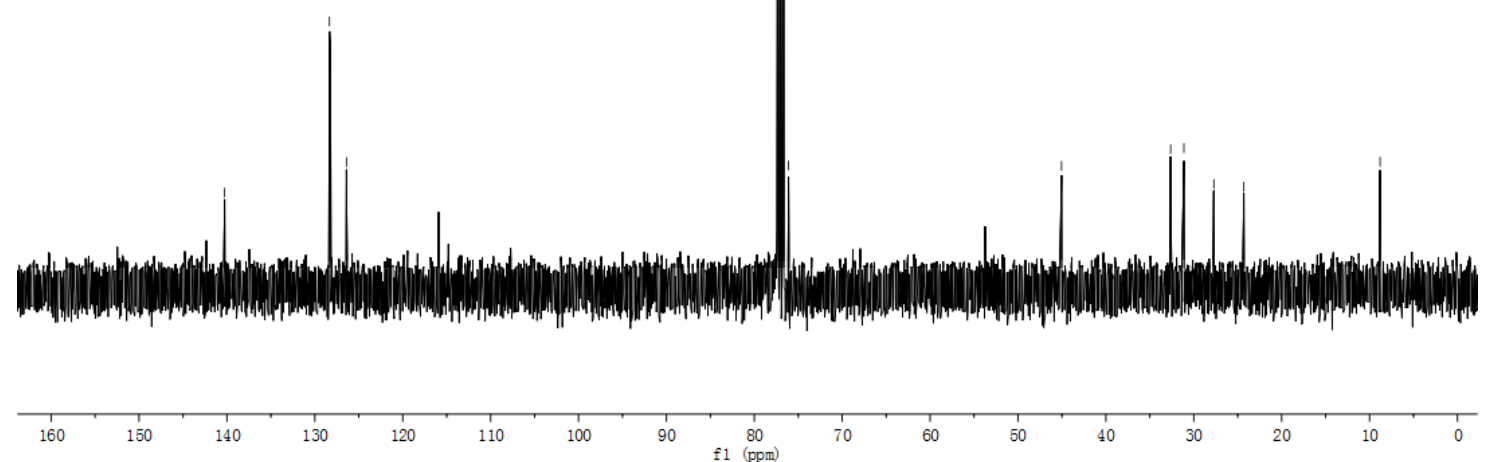

2i HPLC (racemic)

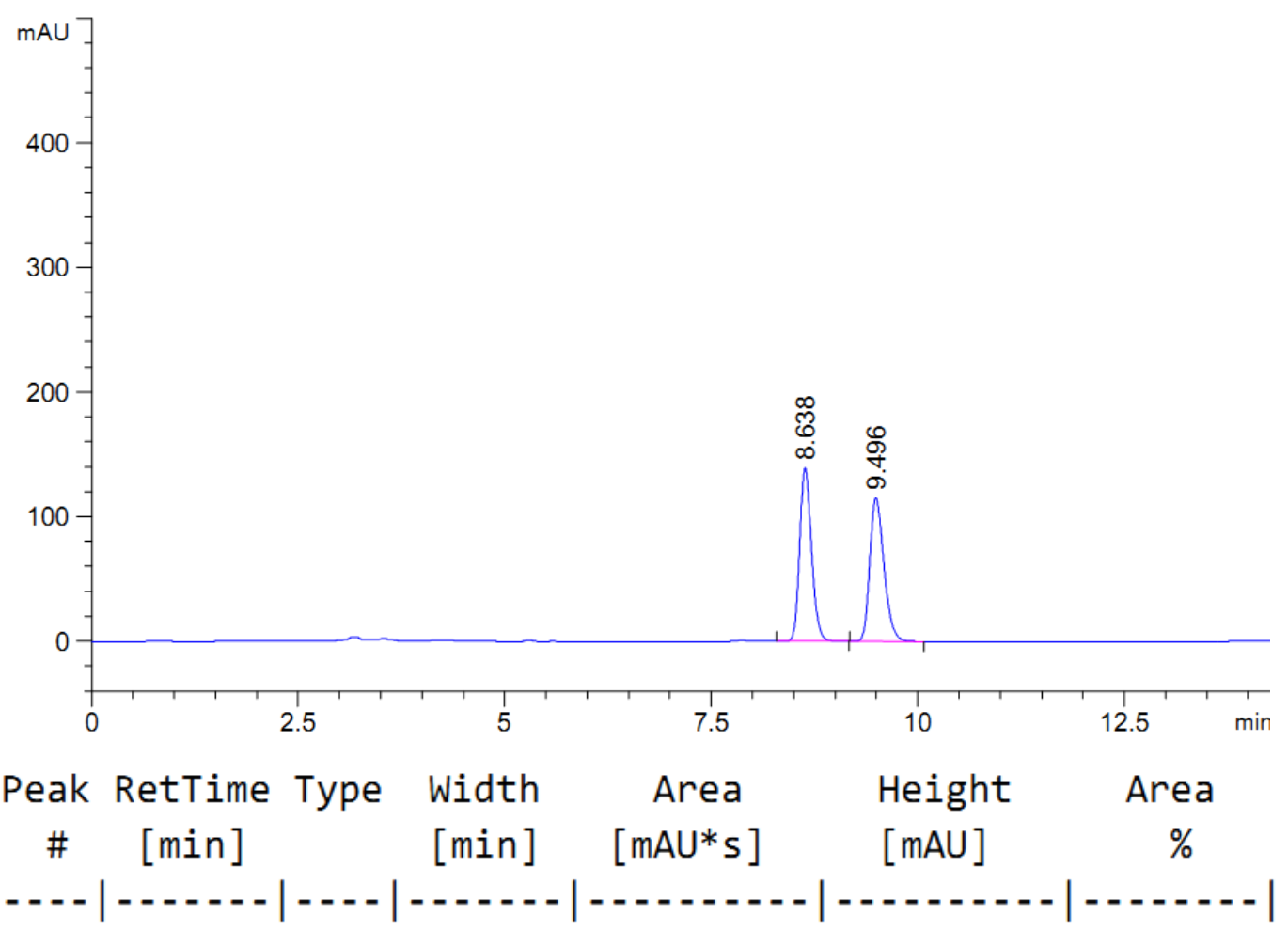

$\begin{array}{llllll}1 & 8.638 \text { BB } & 0.1535 & 1389.01465 & 139.13918 & 50.1805\end{array}$

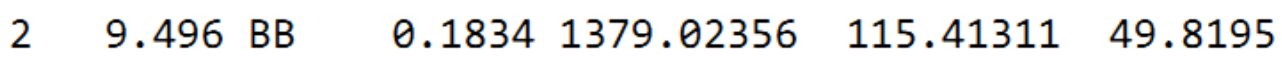




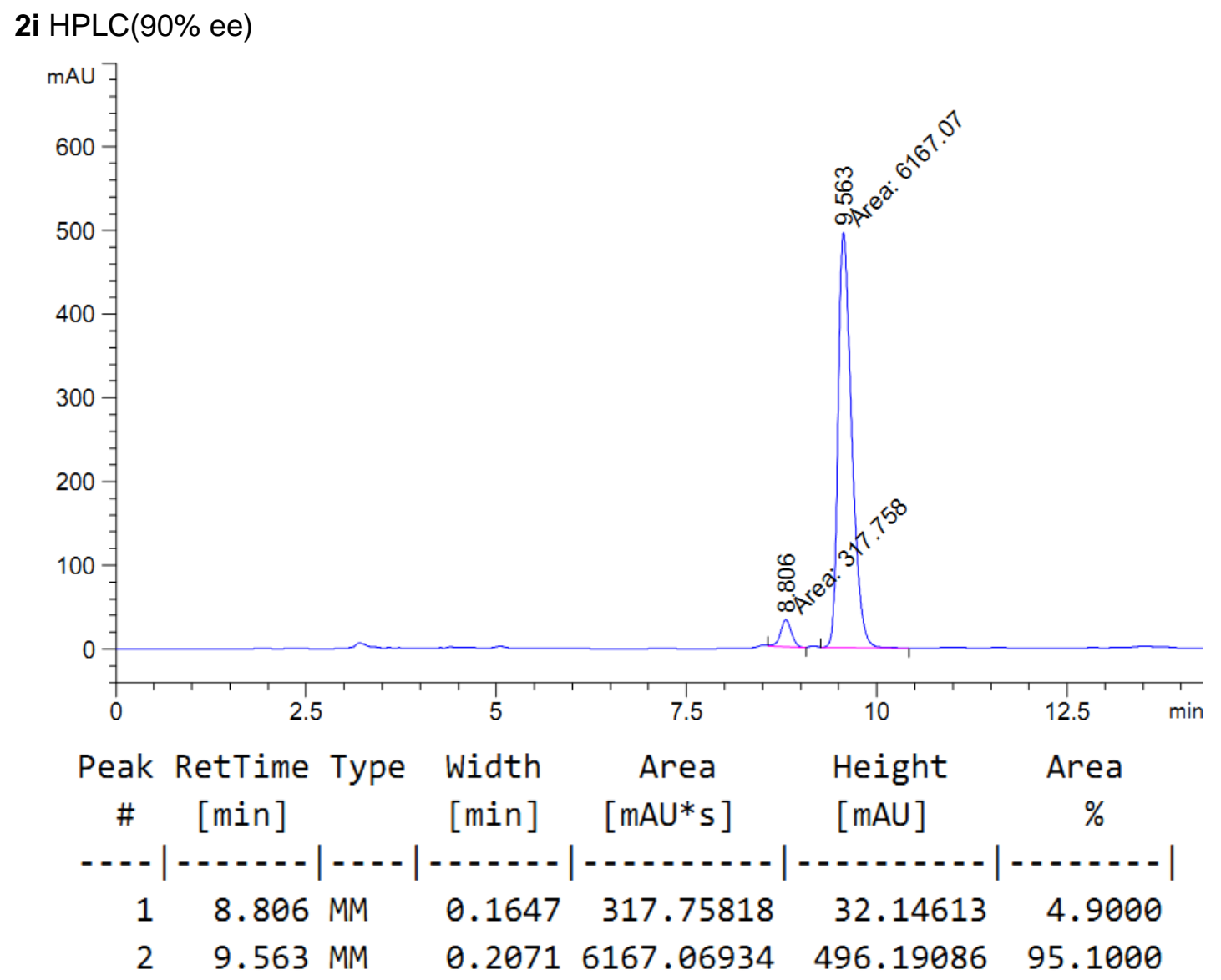

2j ${ }^{1} \mathrm{H}$

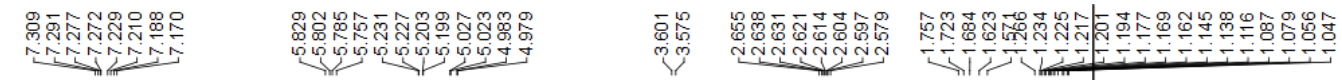

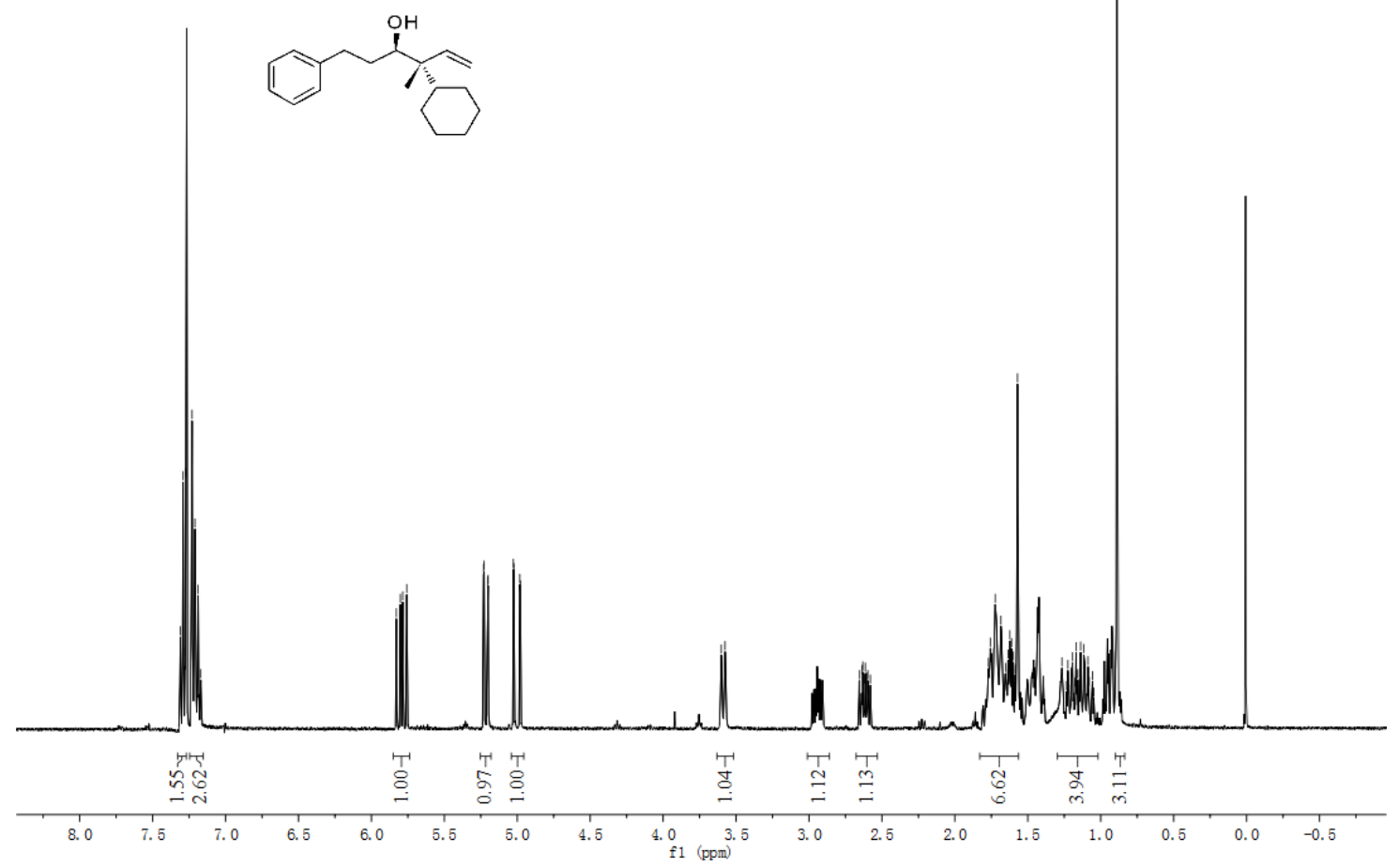


$2{ }^{13} \mathrm{C}$
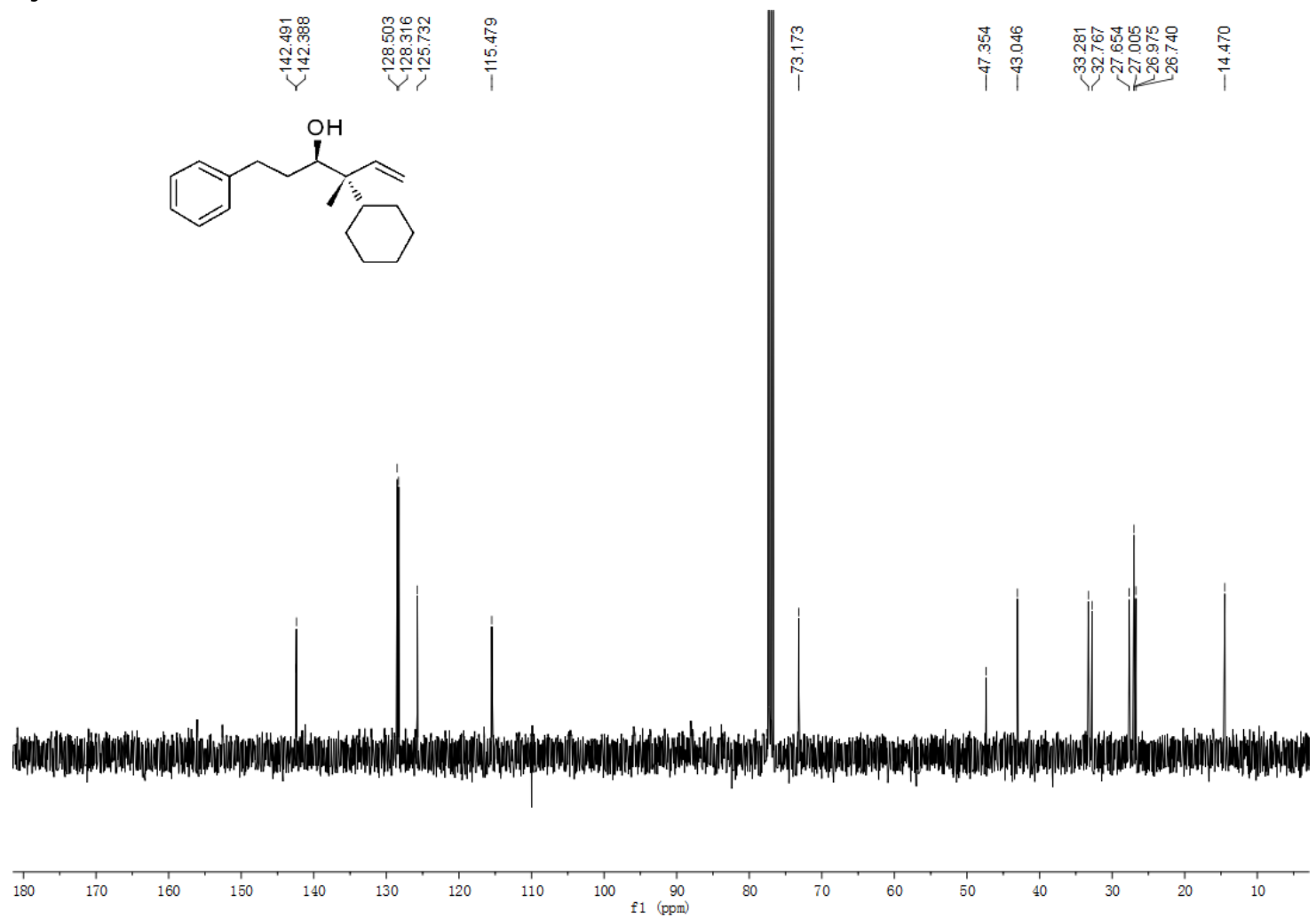

2j HPLC (racemic)

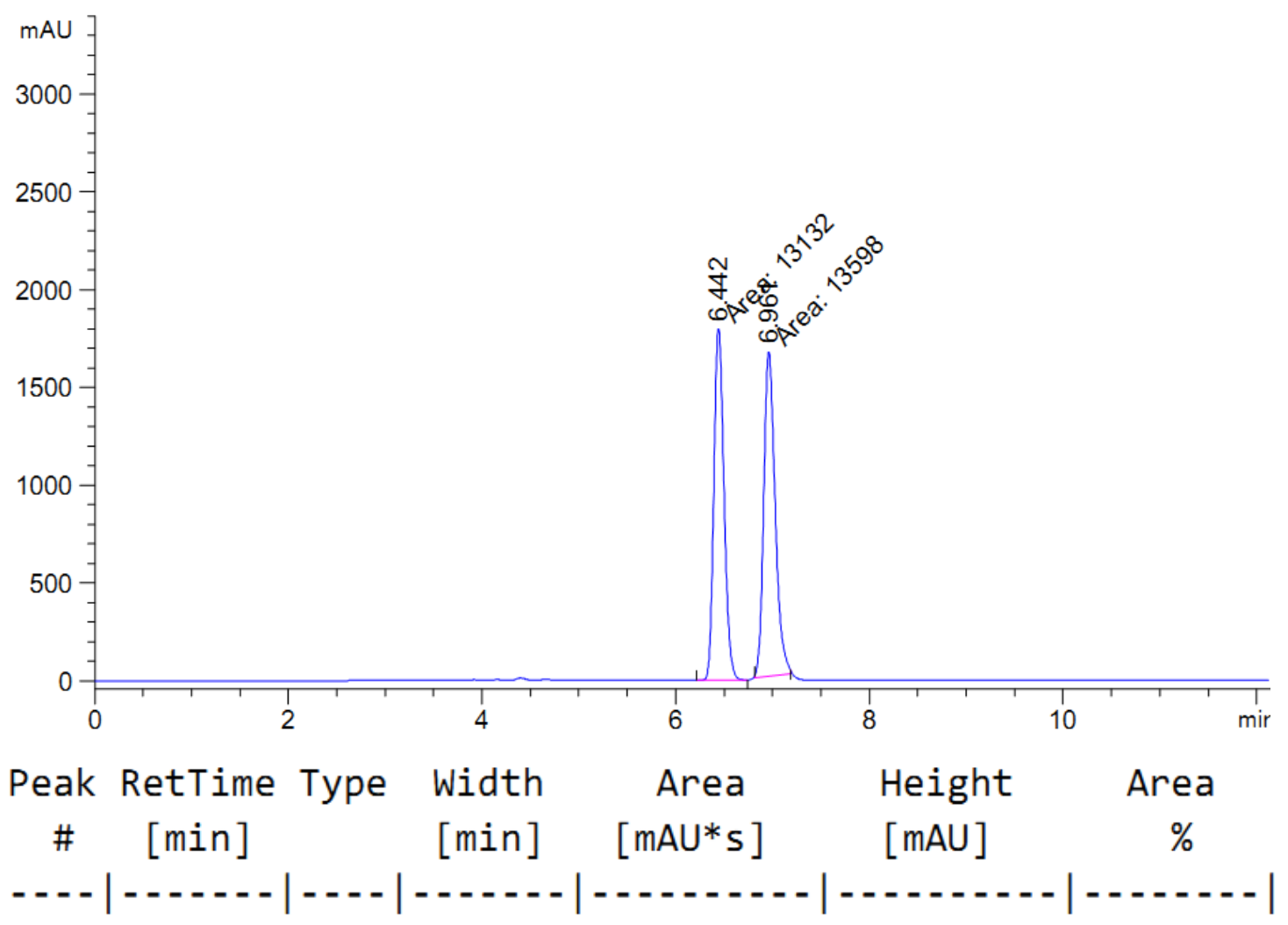

$\begin{array}{llllll}1 & 6.442 \mathrm{MM} & 0.1217 & 1.31320 \mathrm{e} 4 & 1798.22913 & 49.1283\end{array}$

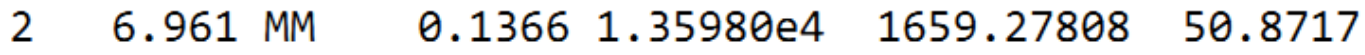




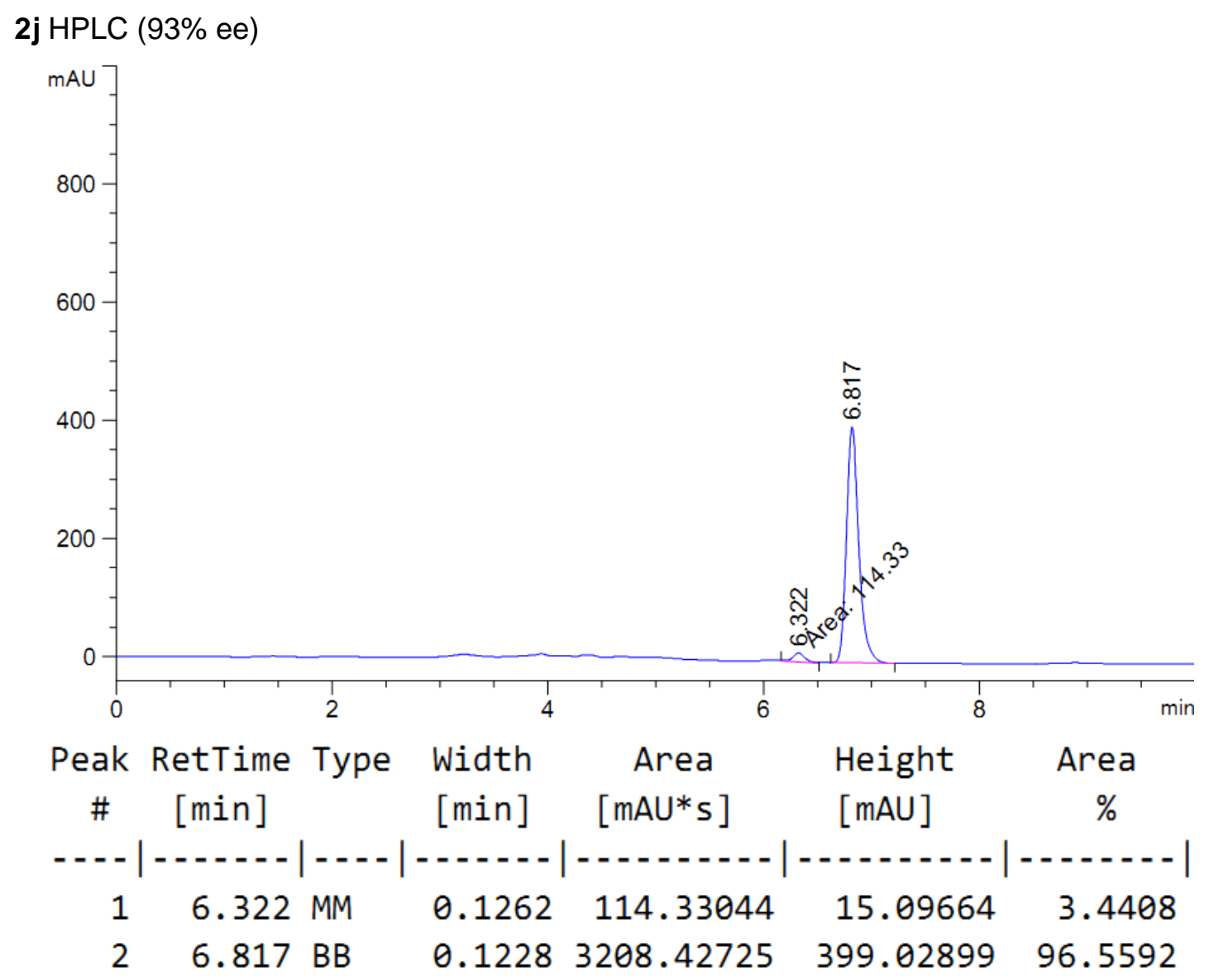

2k ${ }^{1} \mathrm{H}$

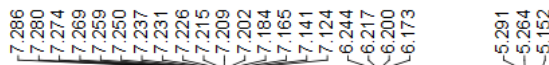<smiles>Oc1ccc(Cl)cc1C(O)c1ccccc1</smiles>

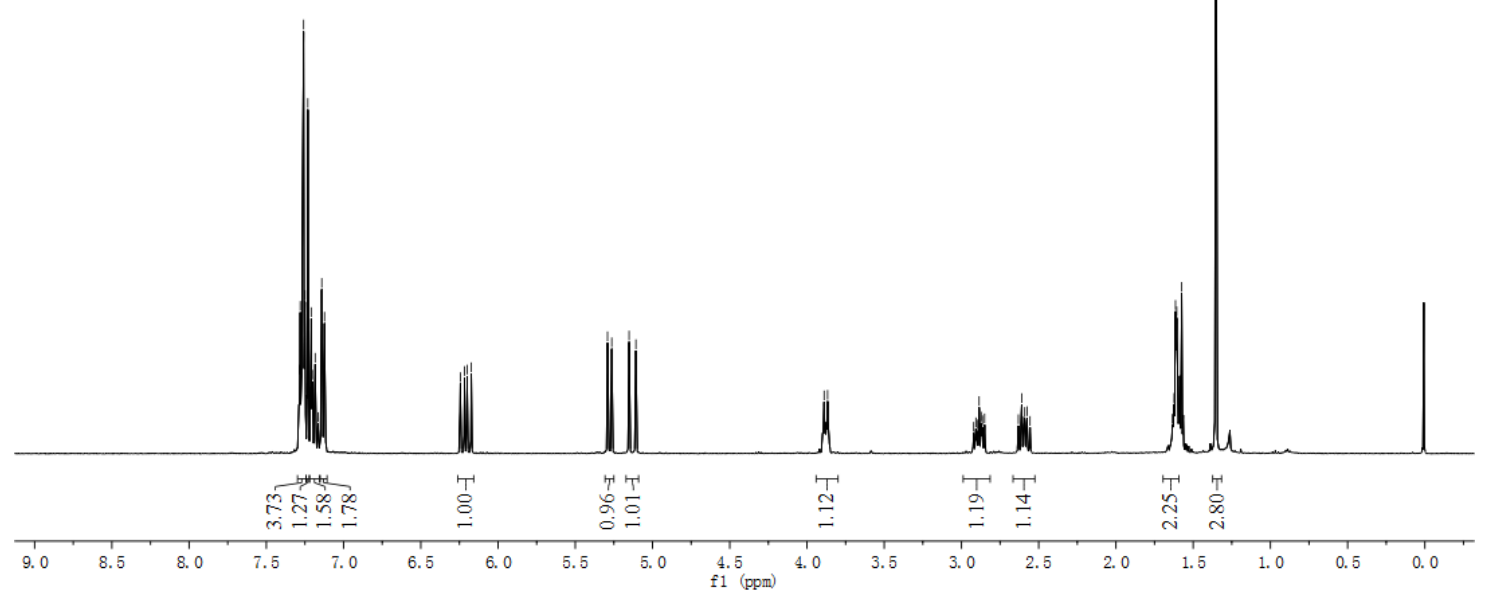


2k ${ }^{13} \mathrm{C}$
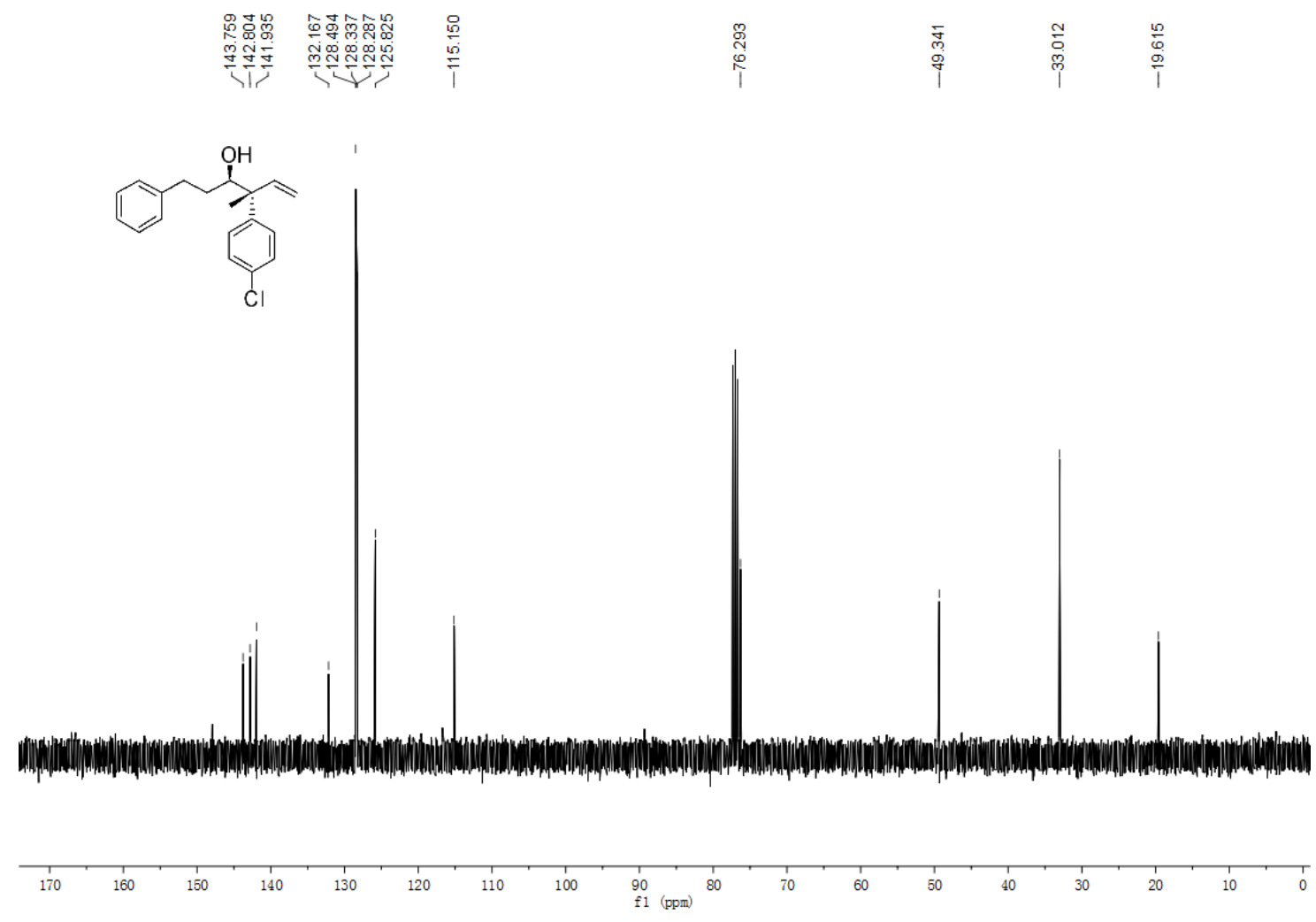

2k HPLC (racemic)

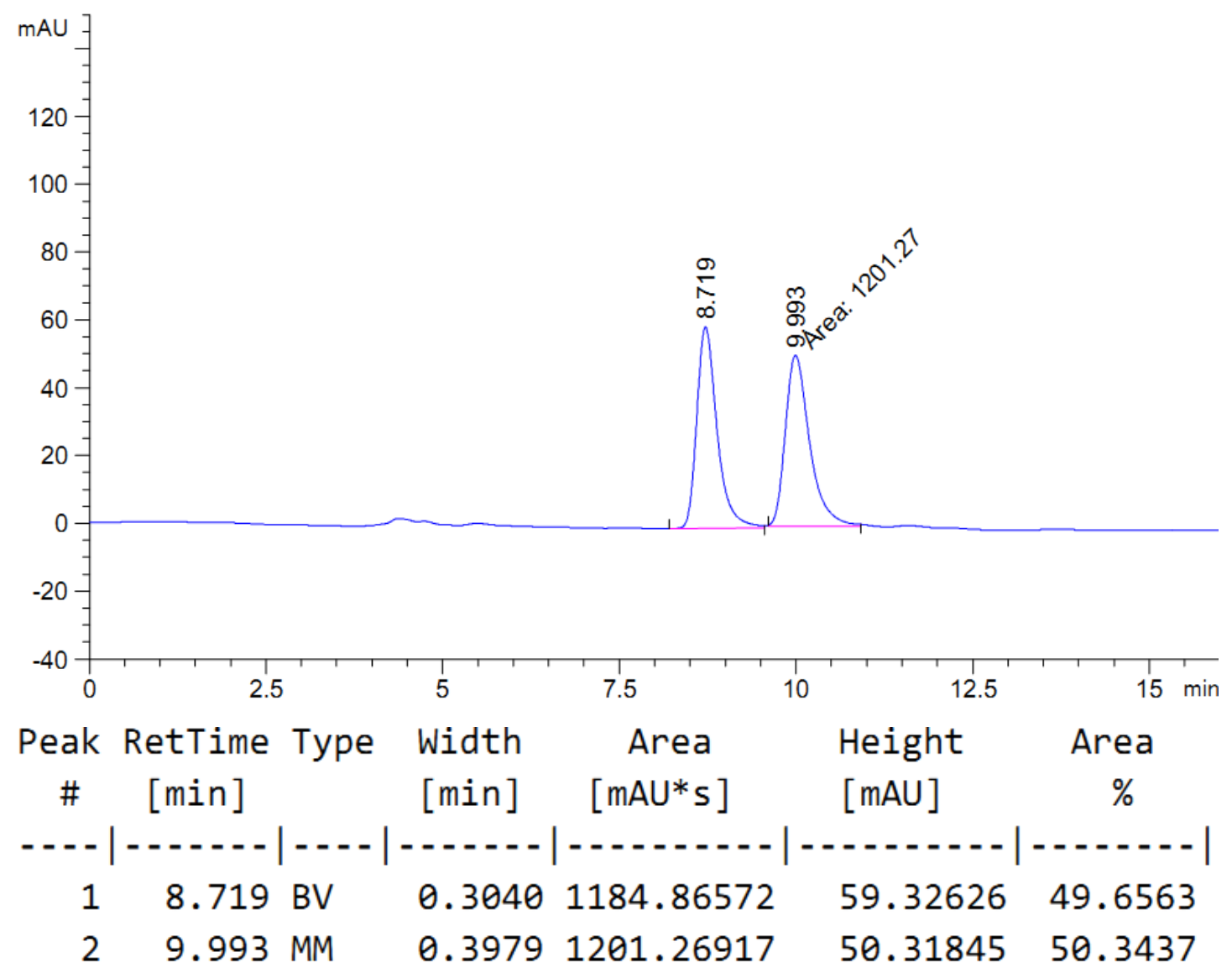


2k HPLC(93\% ee)

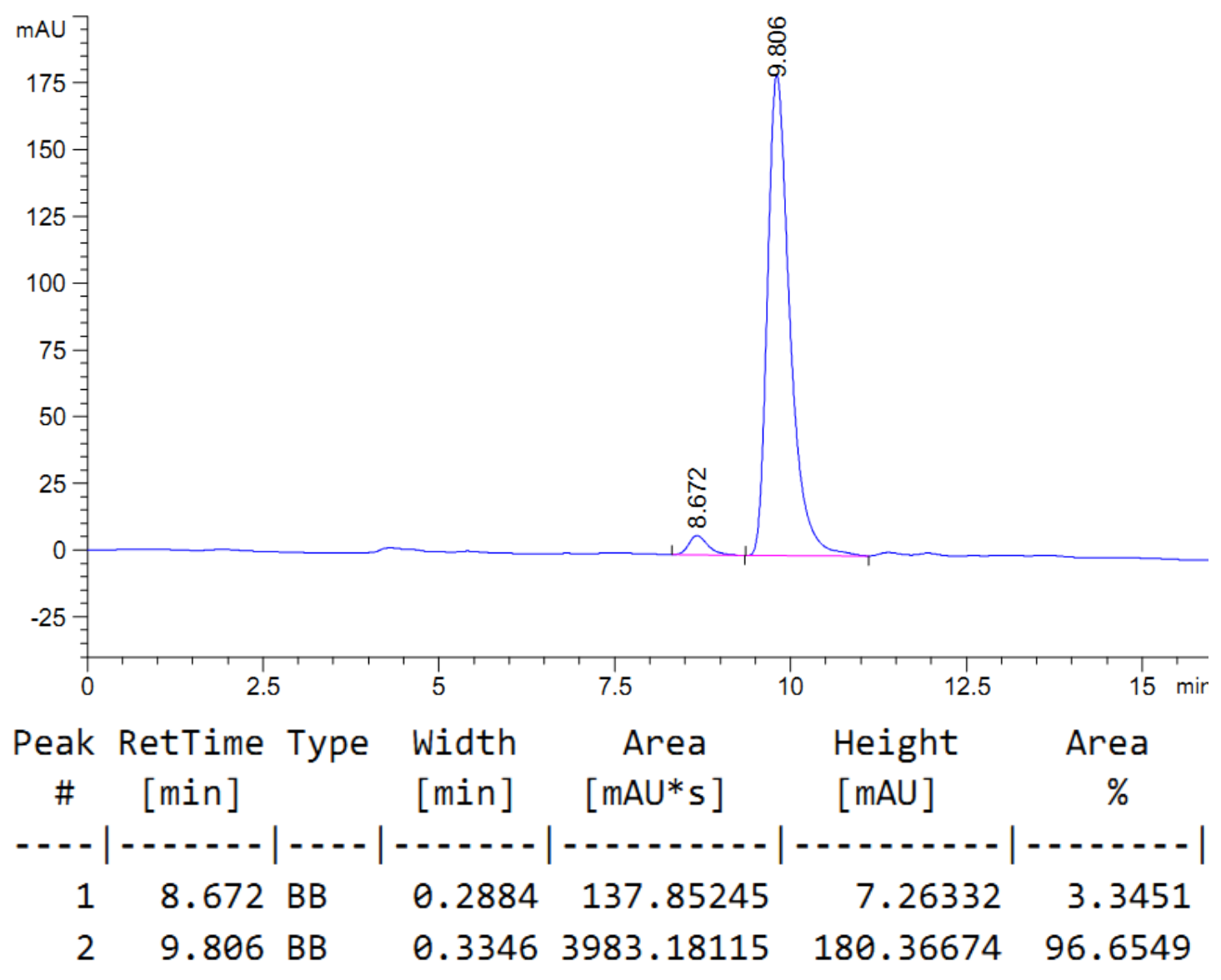
2I ${ }^{1} \mathrm{H}$

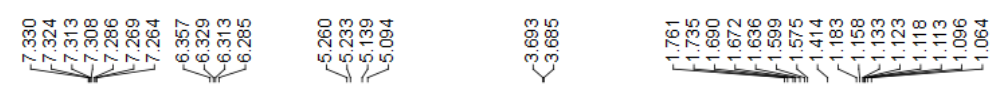<smiles>C=C[C@H](c1ccc(Cl)cc1)C(O)C1CCCCC1</smiles>

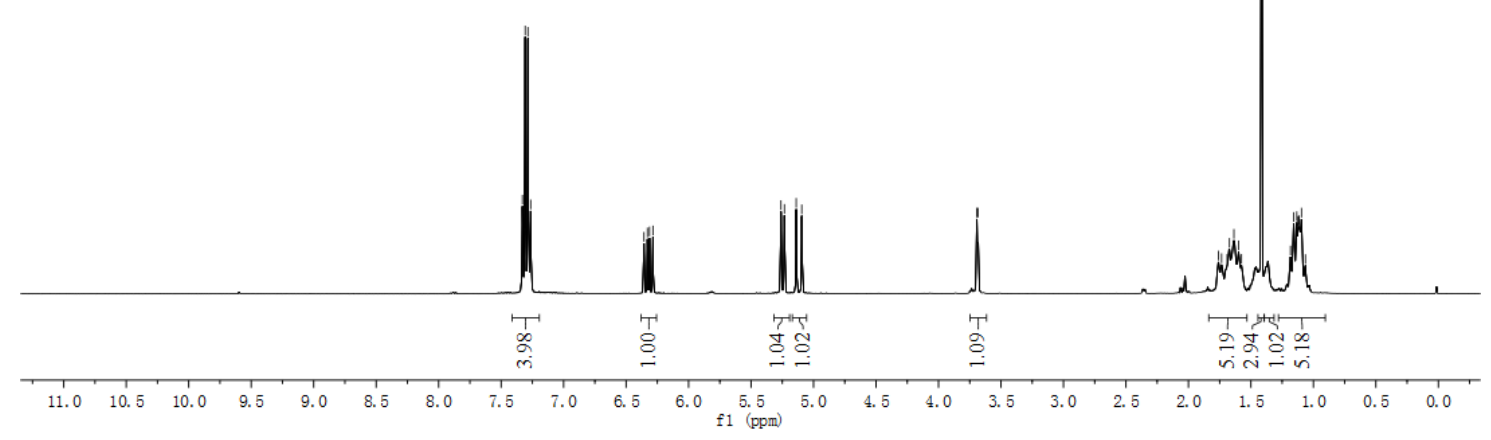


2| ${ }^{13} \mathrm{C}$
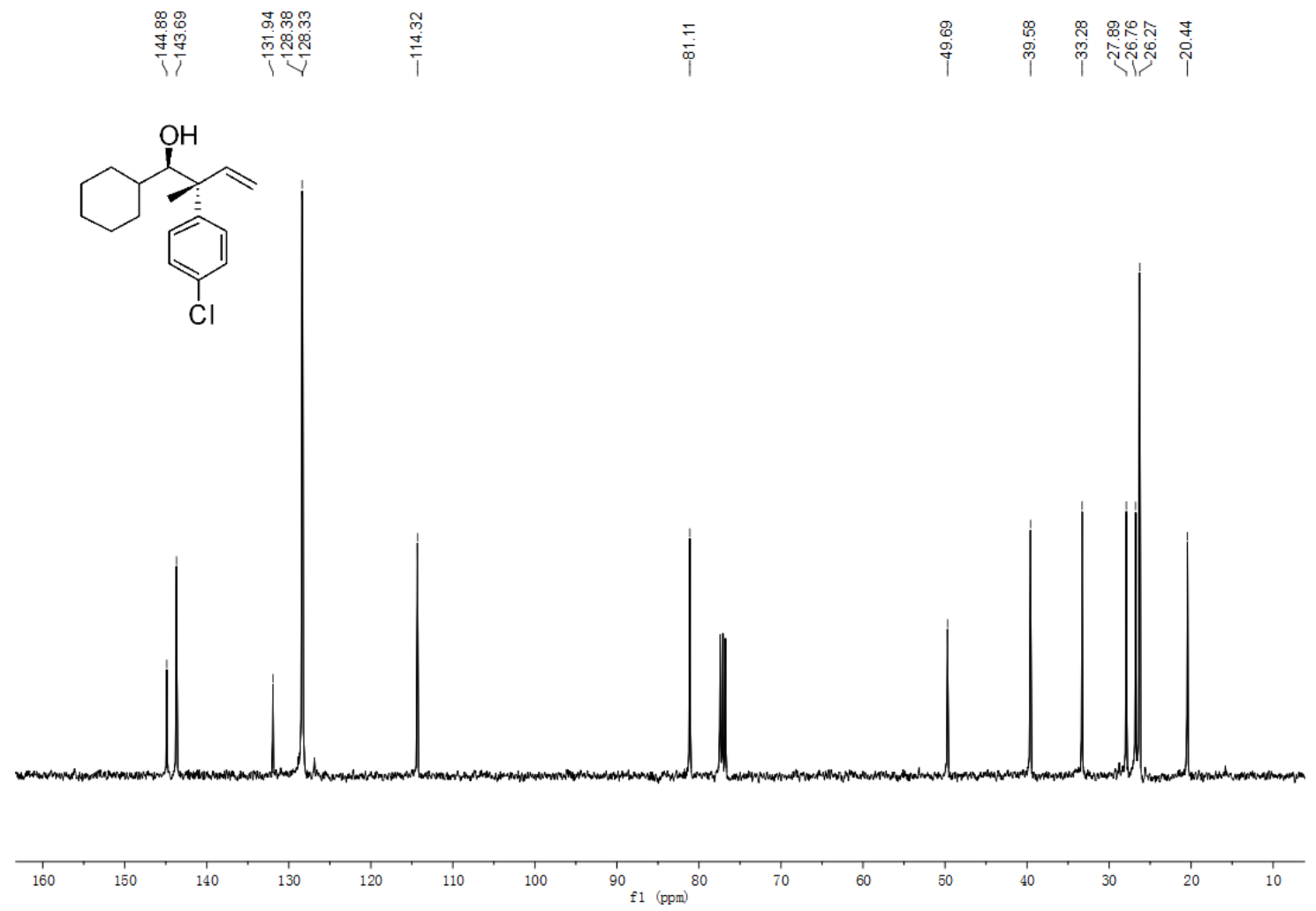

2I HPLC (racemic)

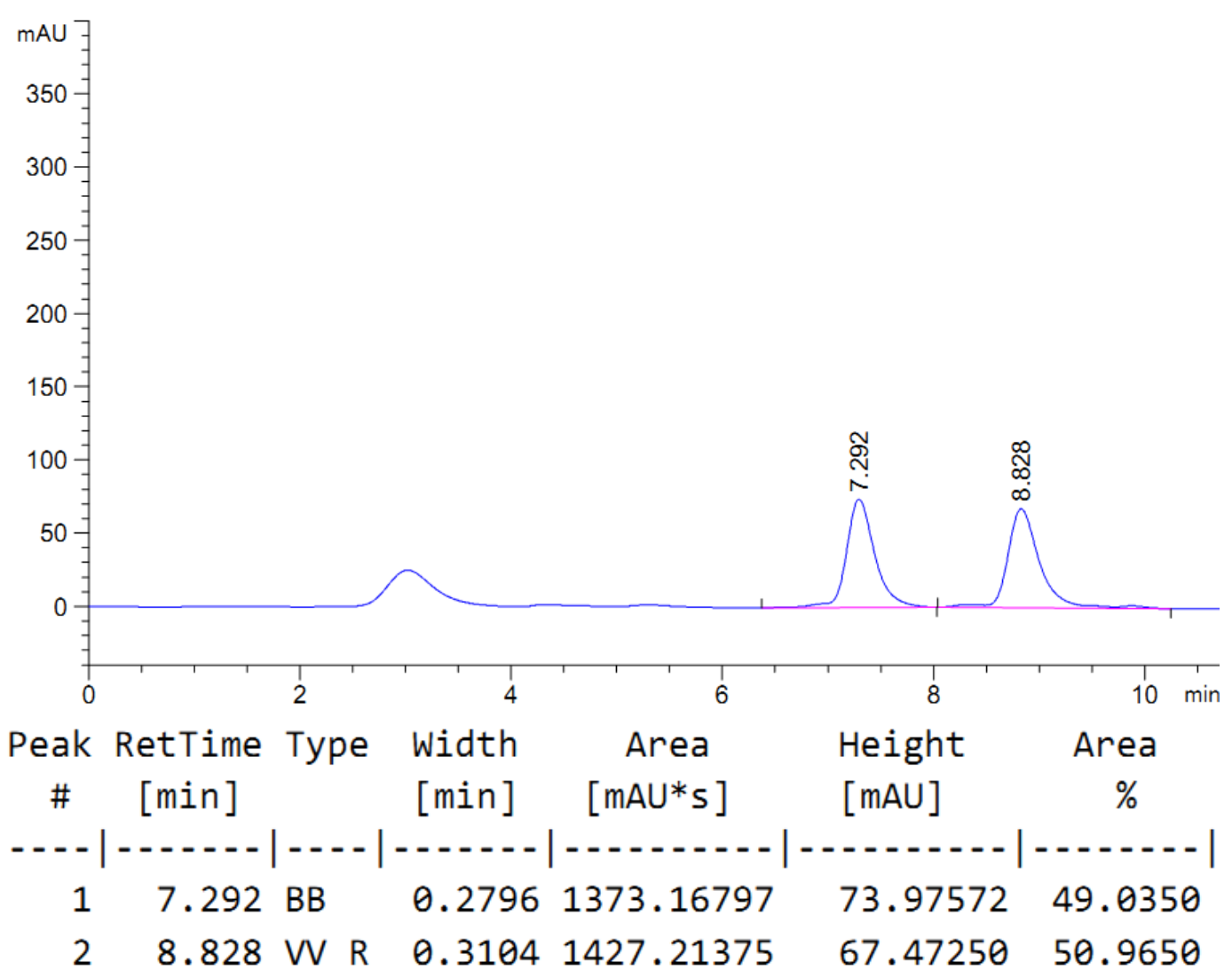




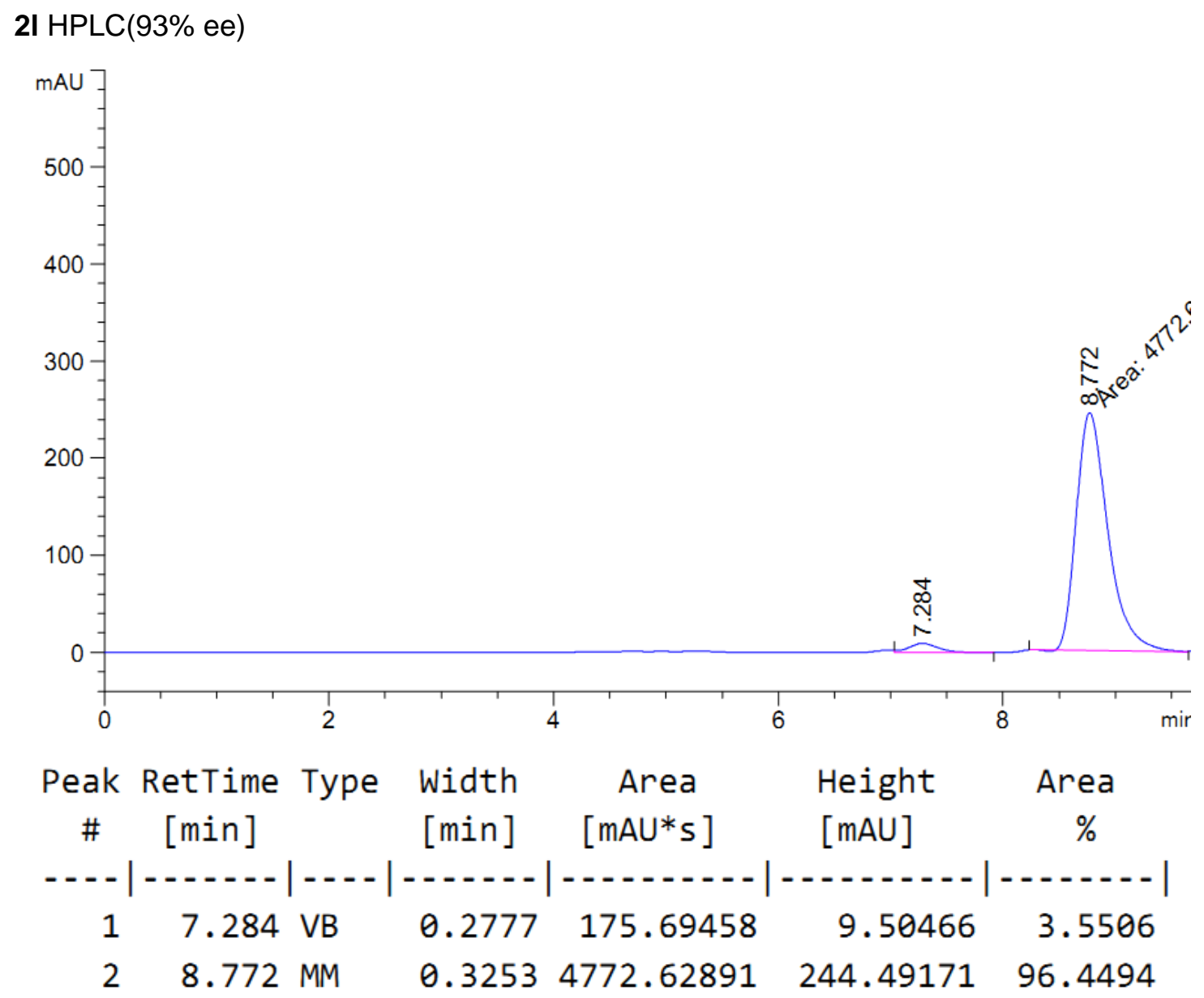

\section{$2 \mathrm{~m}^{1} \mathrm{H}$}

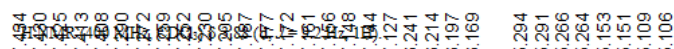

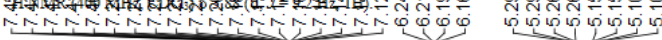

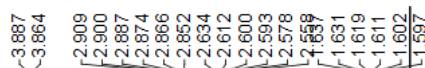<smiles>OC(Cc1ccccc1)Cc1ccc(Br)cc1</smiles>

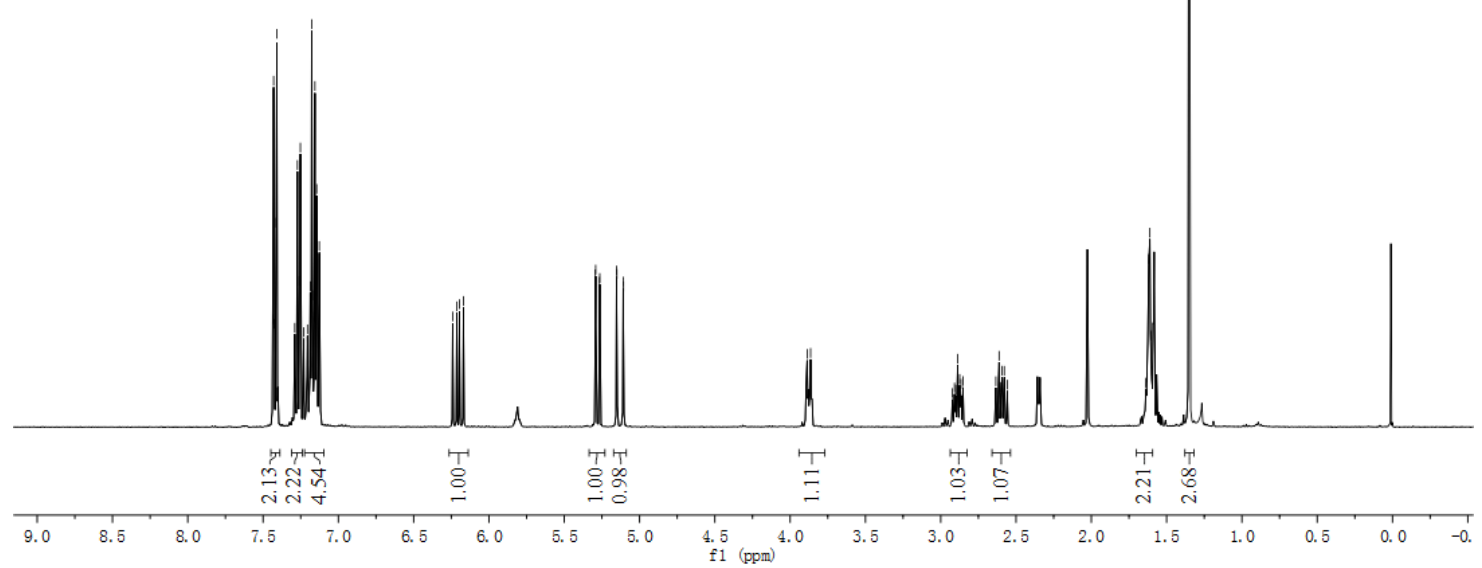


$2 m{ }^{13} \mathrm{C}$

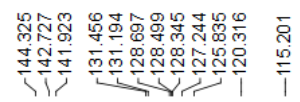

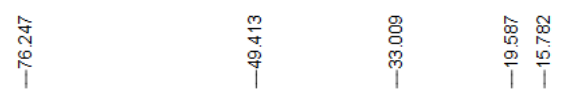<smiles>C=CC(O)(CCc1ccccc1)c1ccc(Br)cc1</smiles>

170

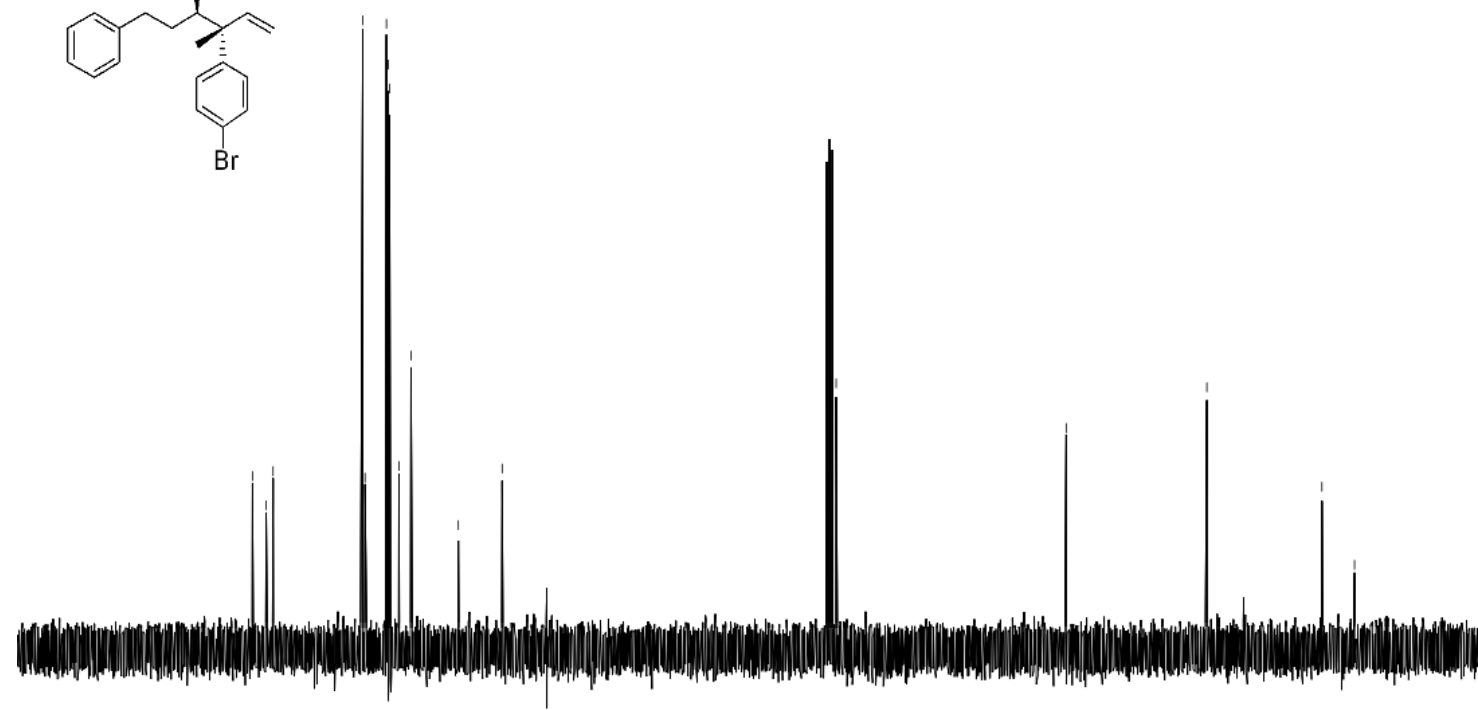

(n)

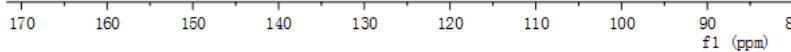

2m HPLC (racemic)

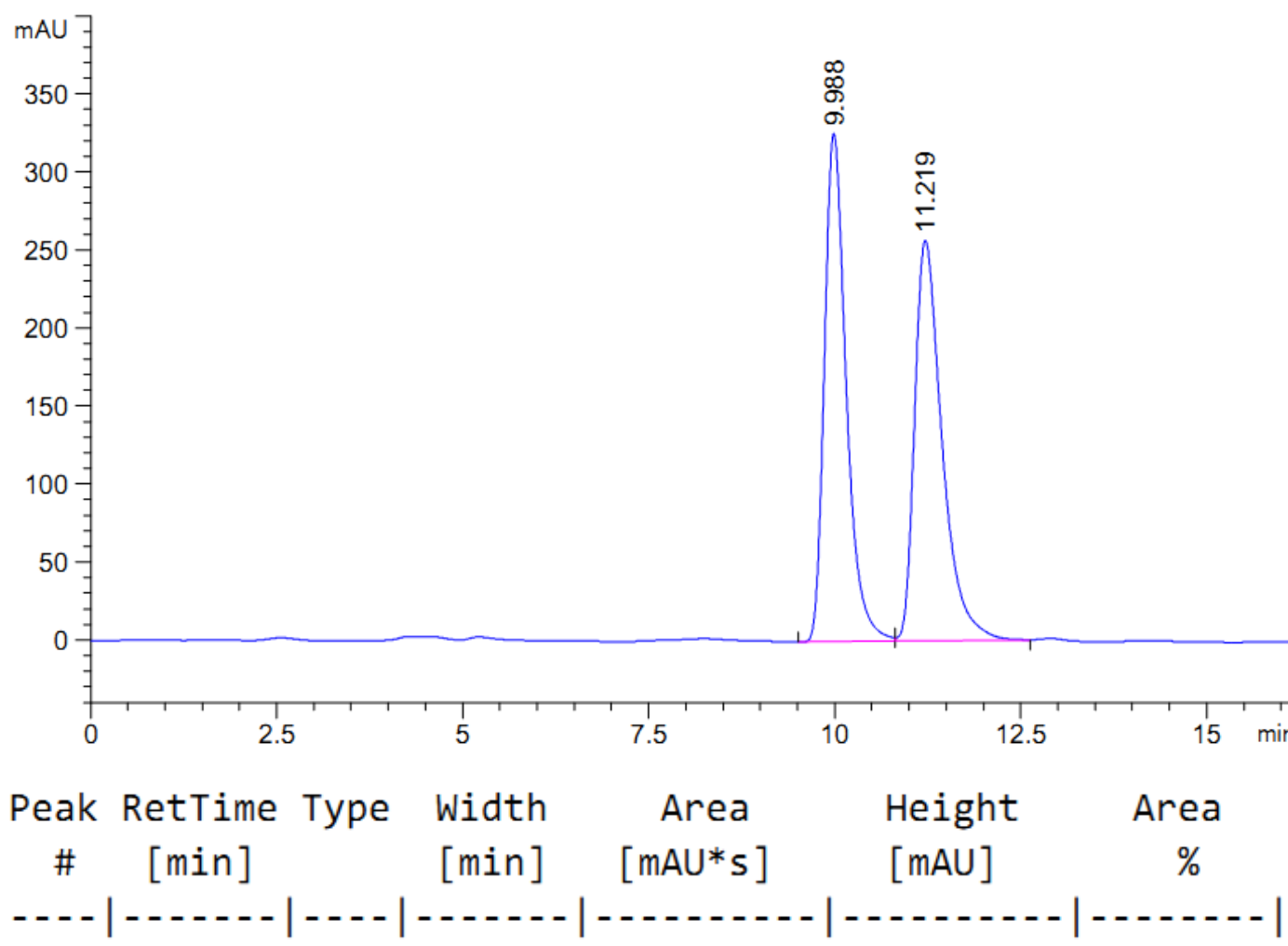

$\begin{array}{lllllll}1 & 9.988 & \text { BV } & 0.3108 & 6640.89014 & 325.77521 & 50.2146\end{array}$

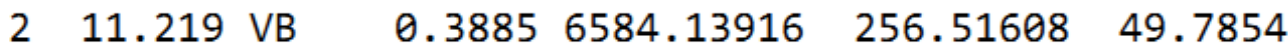




\section{2m HPLC(92\% ee)}

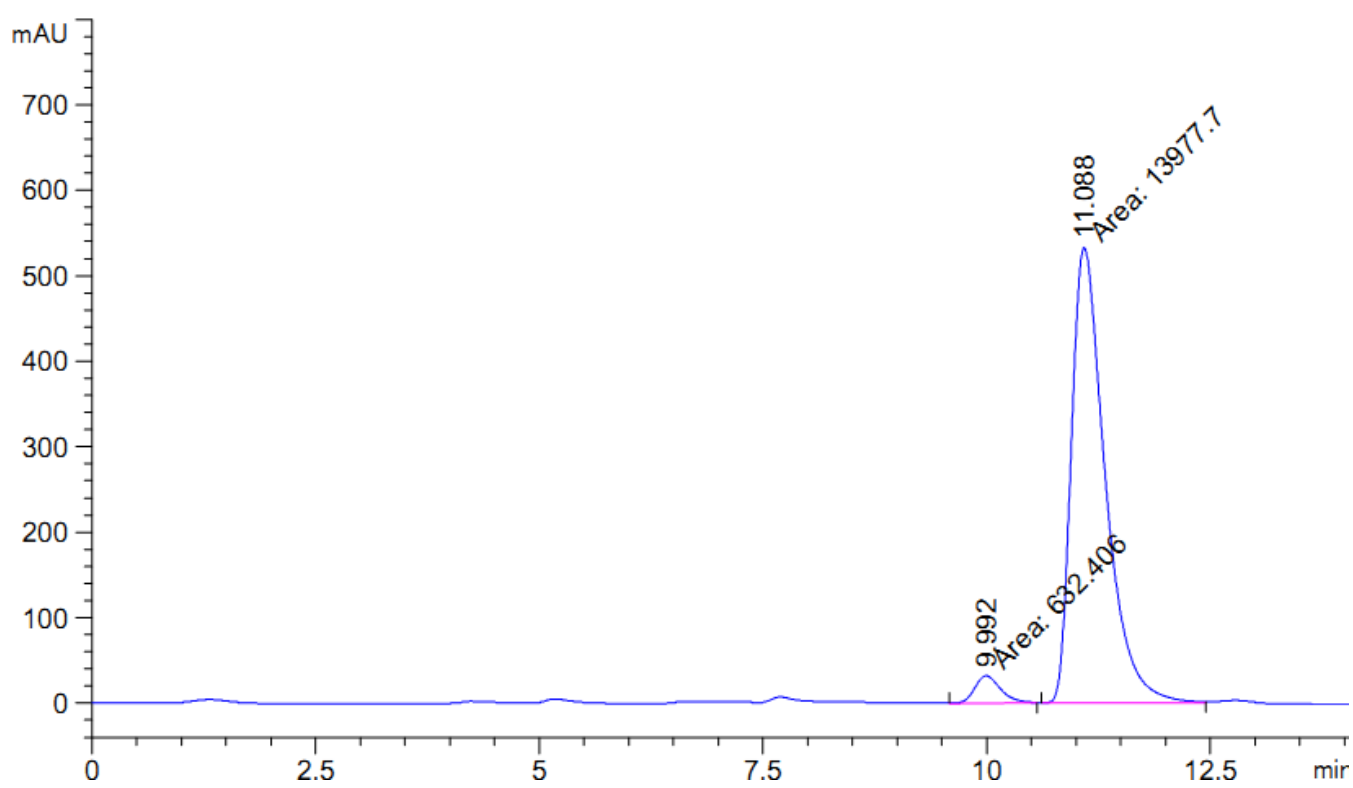

Peak RetTime Type Width Area Height Area

\# $[\mathrm{min}] \quad[\mathrm{min}] \quad[\mathrm{mAU} * \mathrm{~s}] \quad[\mathrm{mAU}] \quad \%$

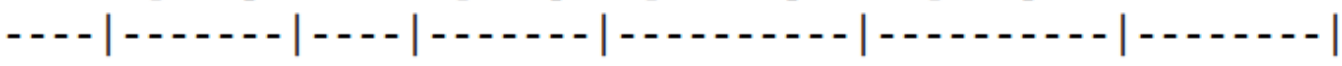
19.992 MM
$0.3299 \quad 632.40594$
31.95218
4.3285
$211.088 \mathrm{MM}$
$0.43721 .39777 \mathrm{e} 4$
$532.90424 \quad 95.6715$

2n ${ }^{1} \mathrm{H}$

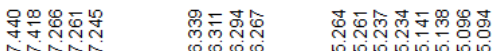

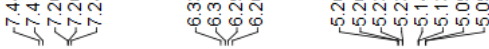

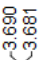

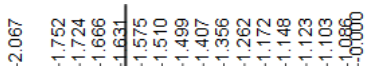<smiles>C=C[C@H](O)C(O)C1CCCCC1</smiles>

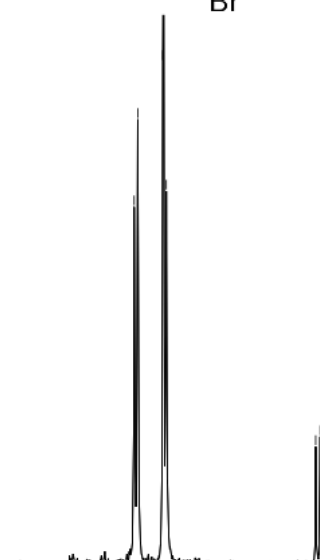

i

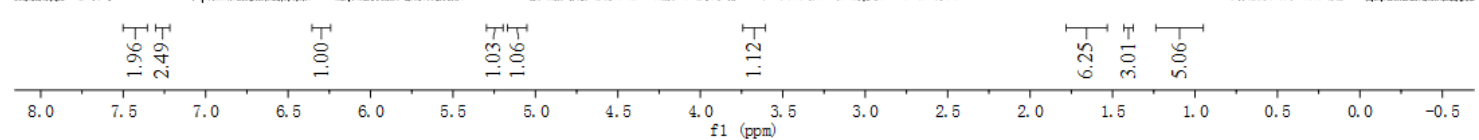


$2 n{ }^{13} \mathrm{C}$<smiles>C=C[C@](O)(c1ccc(Br)cc1)C1CCCCC1</smiles>

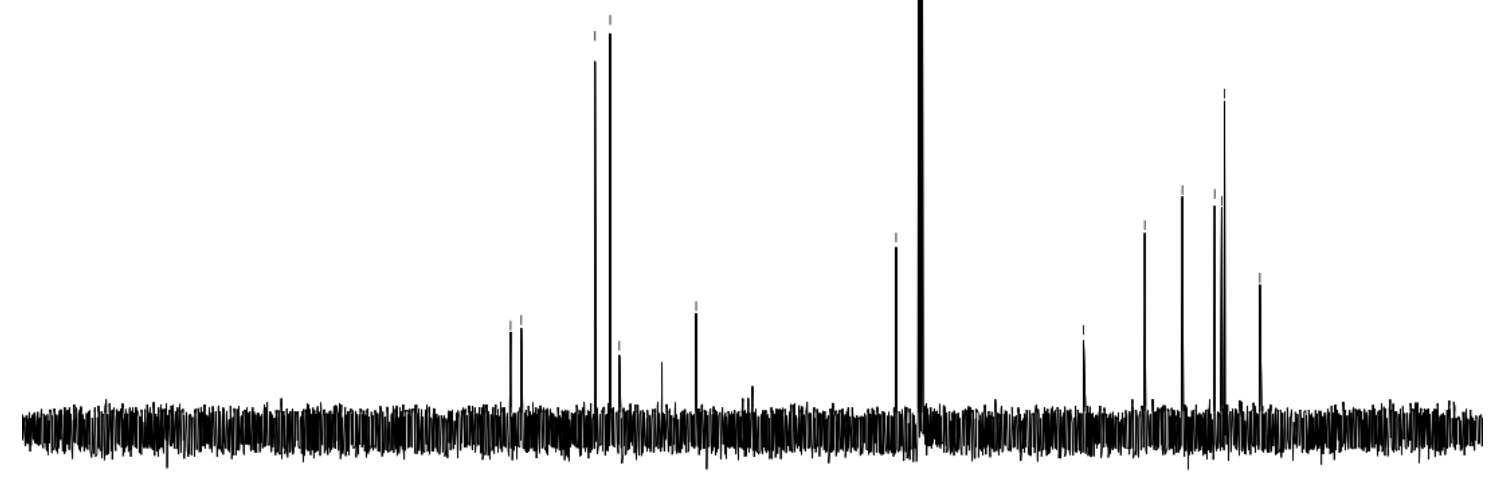

$\begin{array}{lllllllllllll}220 & 210 & 200 & 190 & 180 & 170 & 160 & 150 & 140 & 130 & 120 & 110 & 100 \\ \mathrm{f} 1 & (\mathrm{ppm})\end{array}$

2n HPLC (racemic)

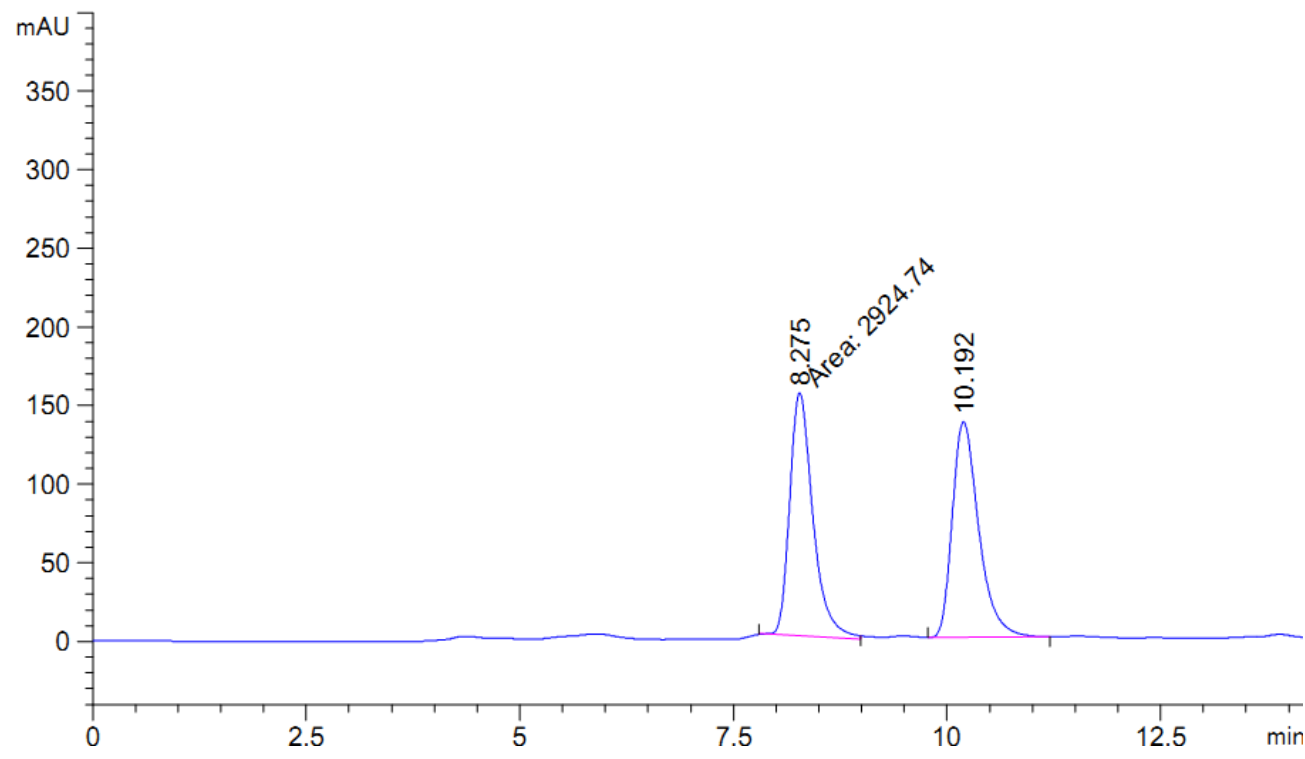

Peak RetTime Type Width Area Height Area

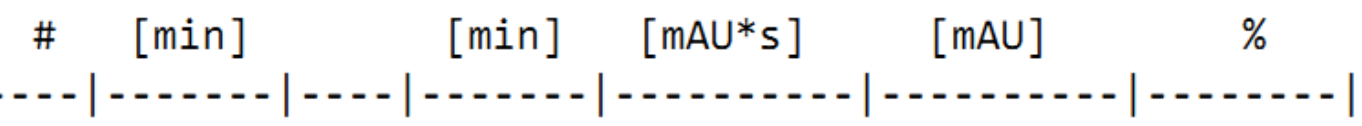

$\begin{array}{lllllll}1 & 8.275 & \text { MM } & 0.3156 & 2924.73511 & 154.45918 & 49.6791\end{array}$

$\begin{array}{lllllll}2 & 10.192 & \text { VB } & 0.3287 & 2962.51758 & 137.32736 & 50.3209\end{array}$ 
2n HPLC(92\% ee)

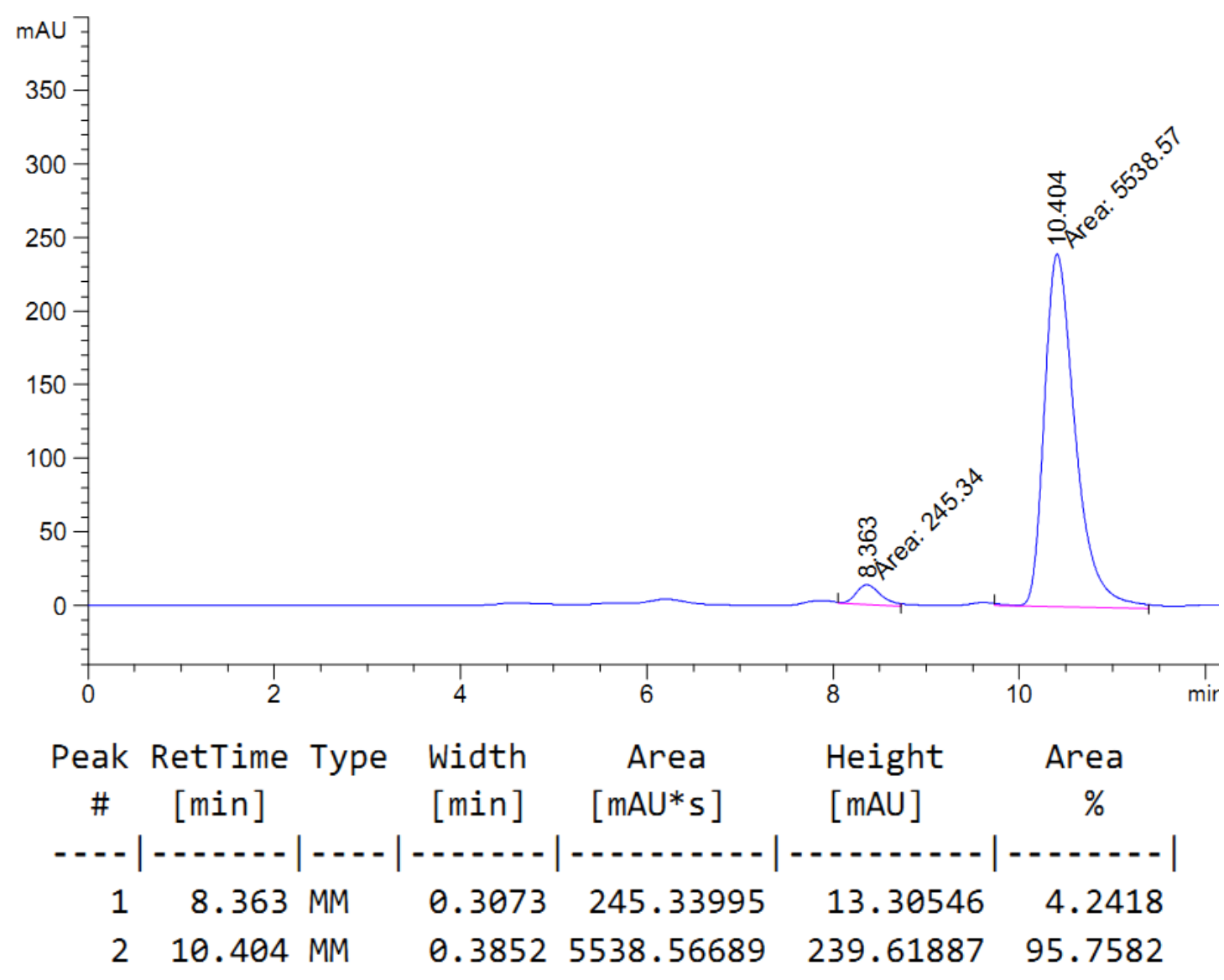

$20{ }^{1} \mathrm{H}$

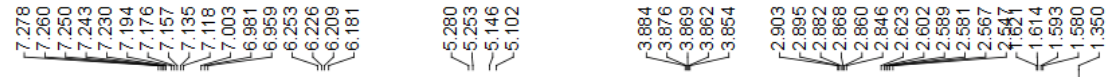

(1)

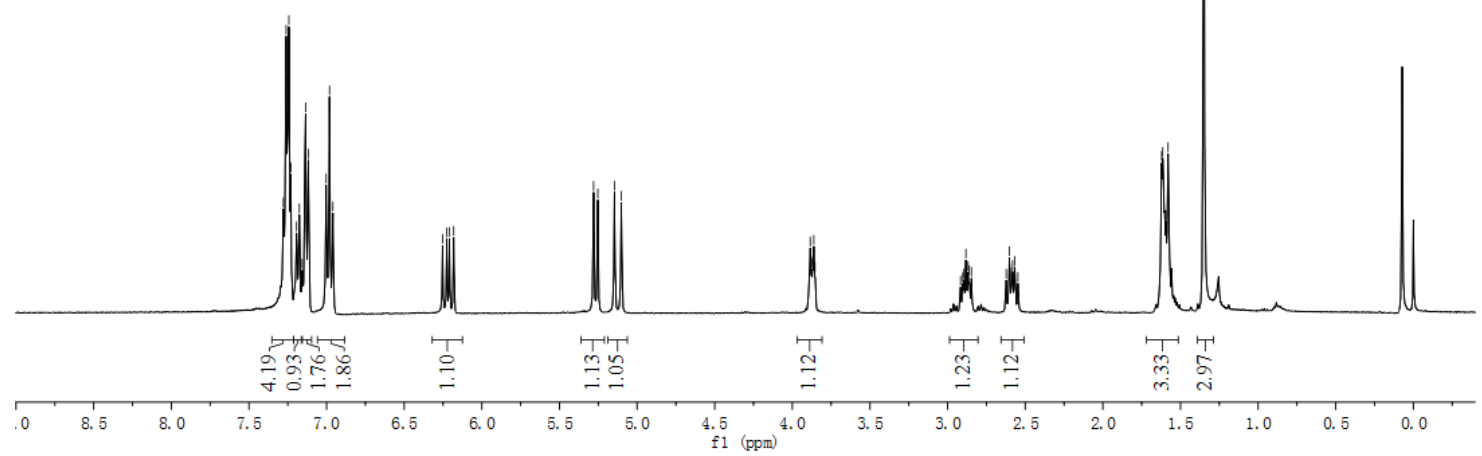


$20{ }^{13} \mathrm{C}$
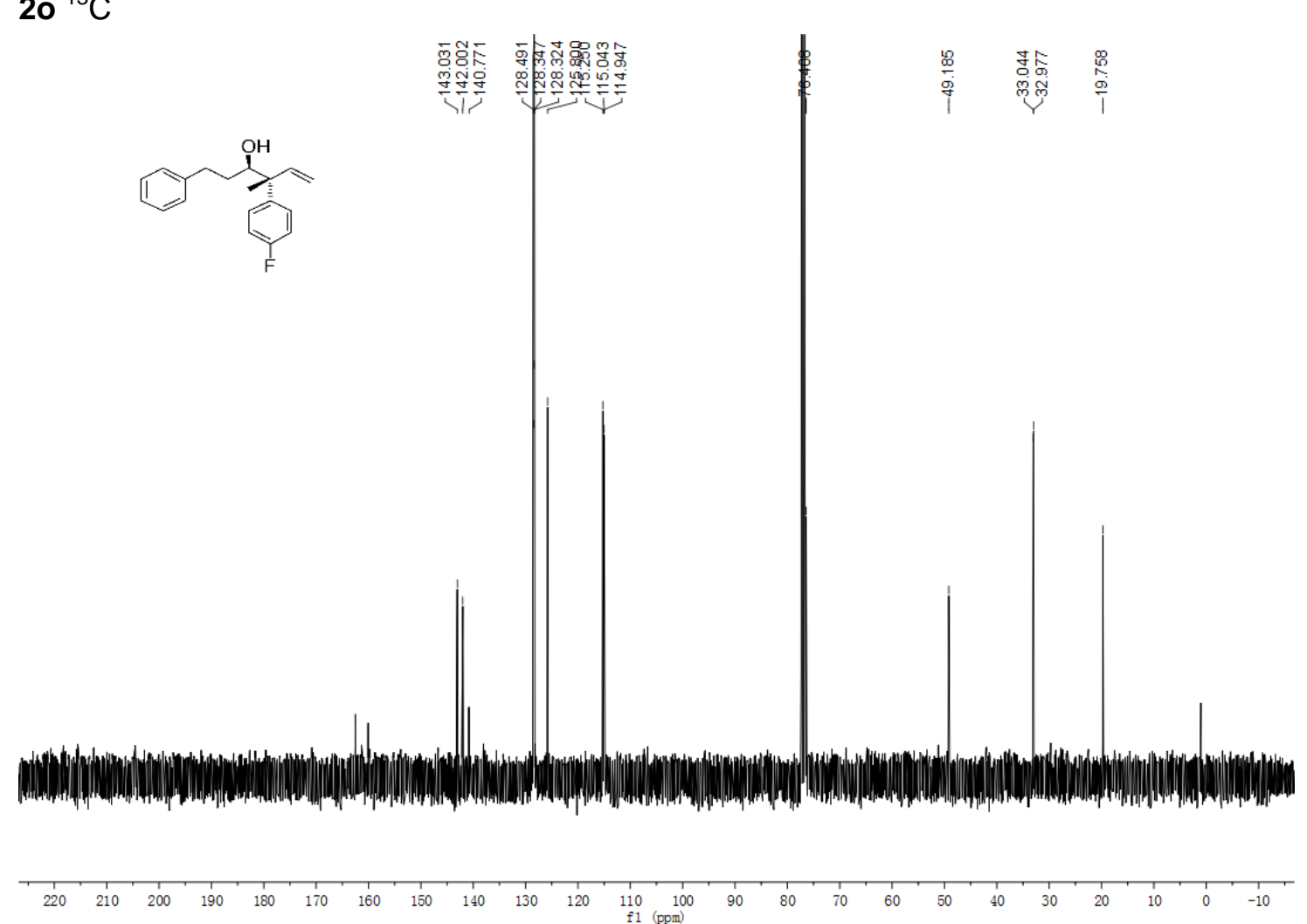

20 HPLC (racemic)

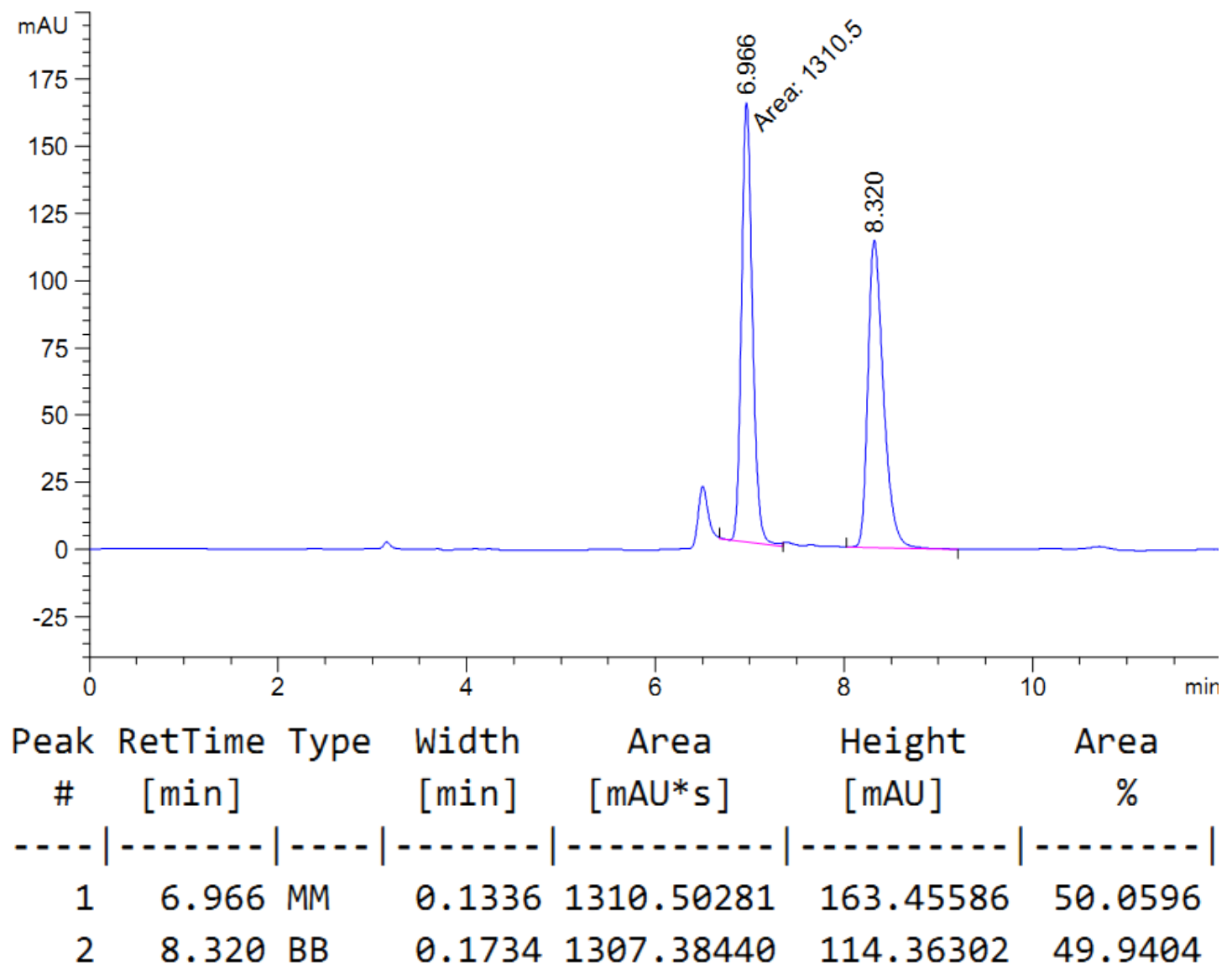


20 HPLC(94\% ee)

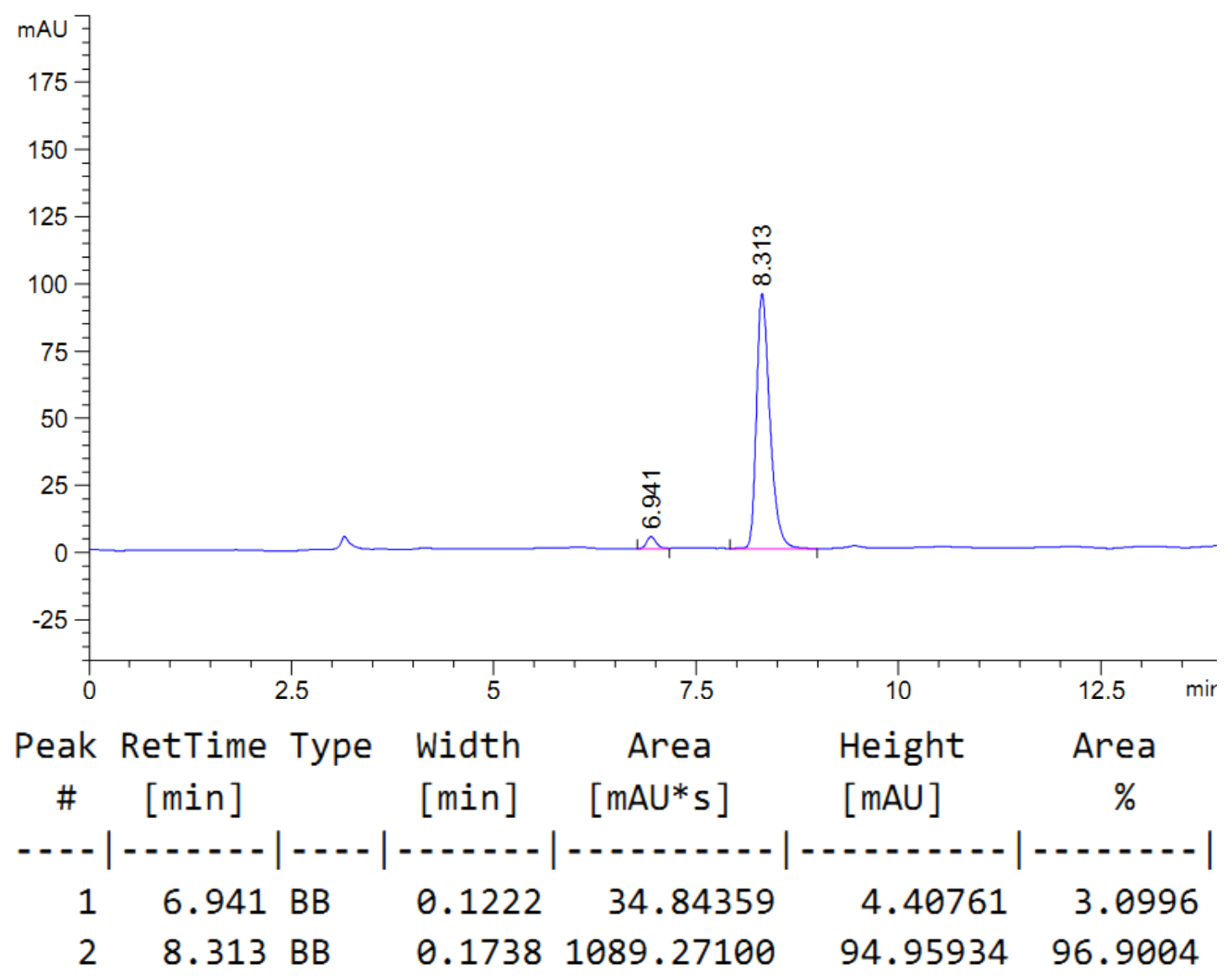

$2 p^{1} \mathrm{H}$

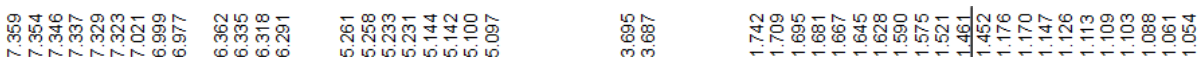<smiles>C=CC(O)[C@H](c1ccc(F)cc1)C1CCCCC1</smiles>

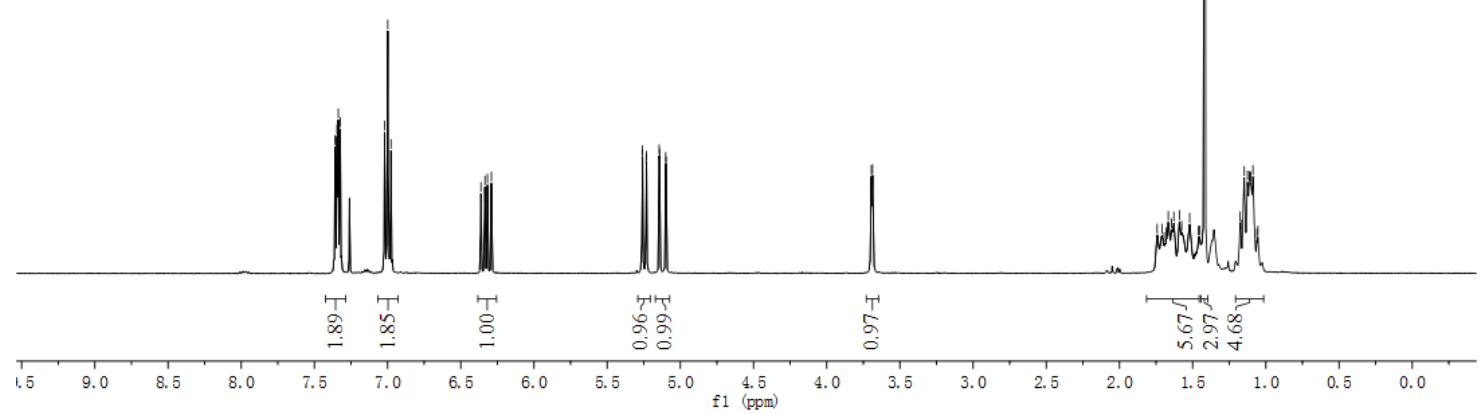


$2 p{ }^{13} \mathrm{C}$
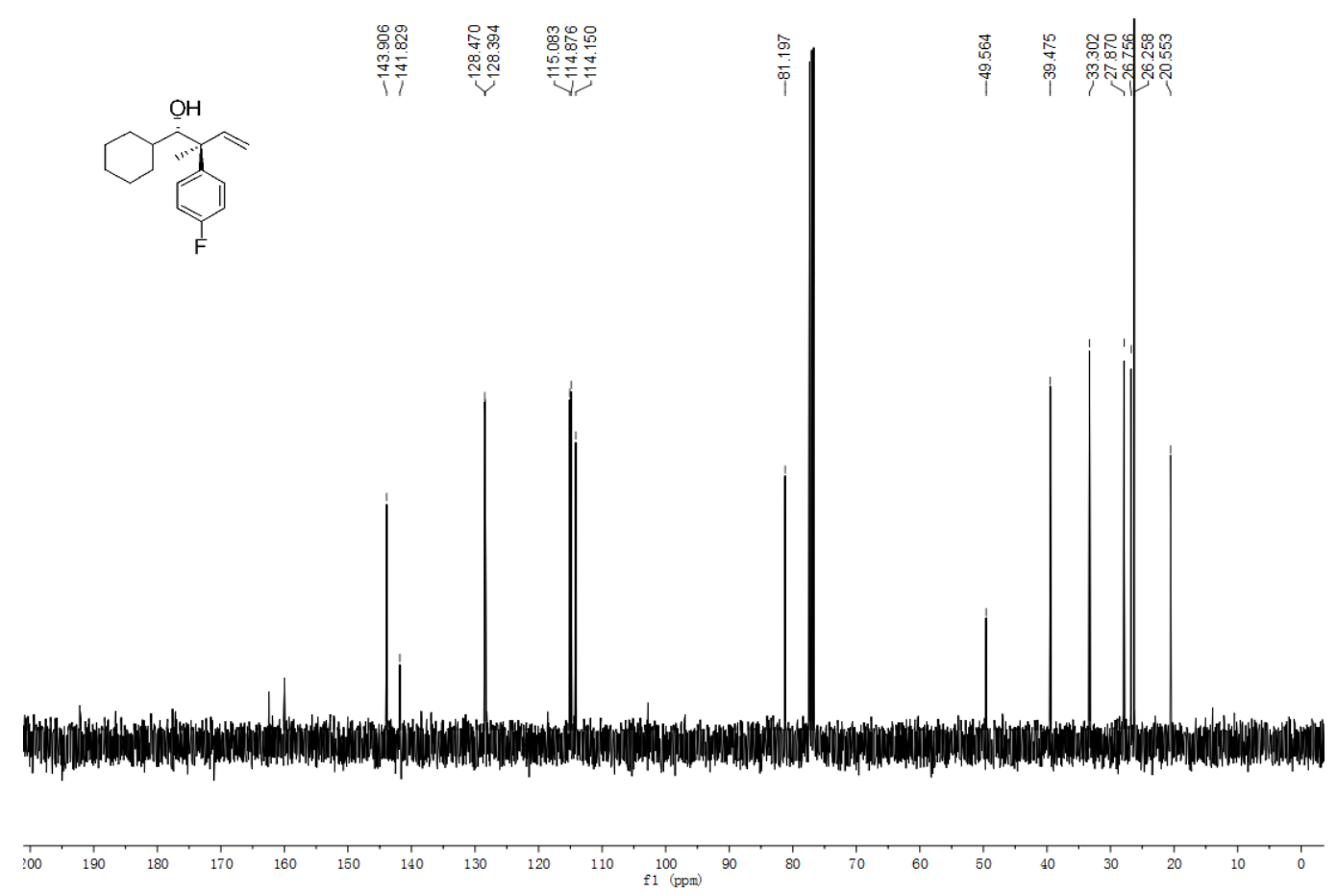

2p HPLC (racemic)

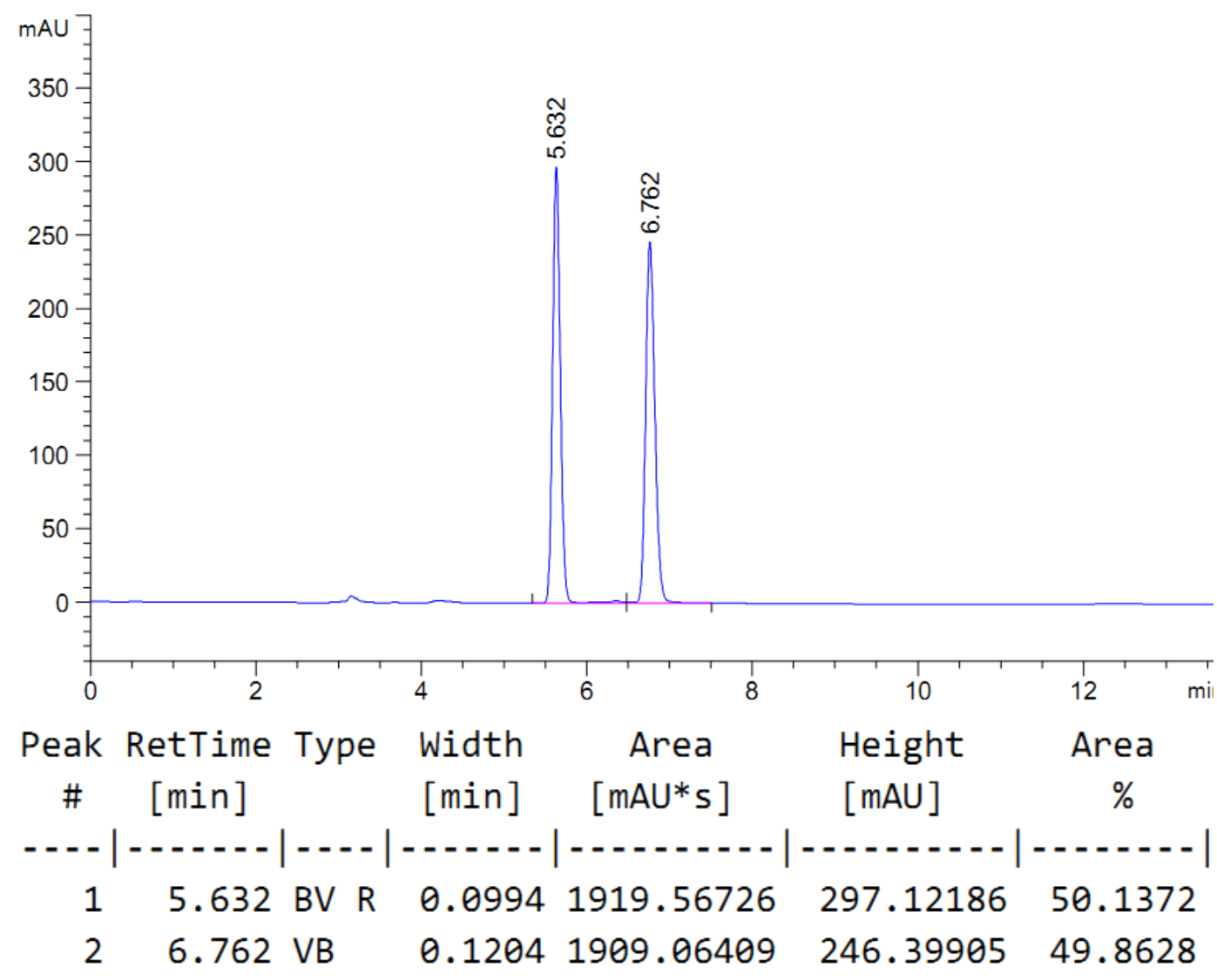


2p HPLC(95\% ee)

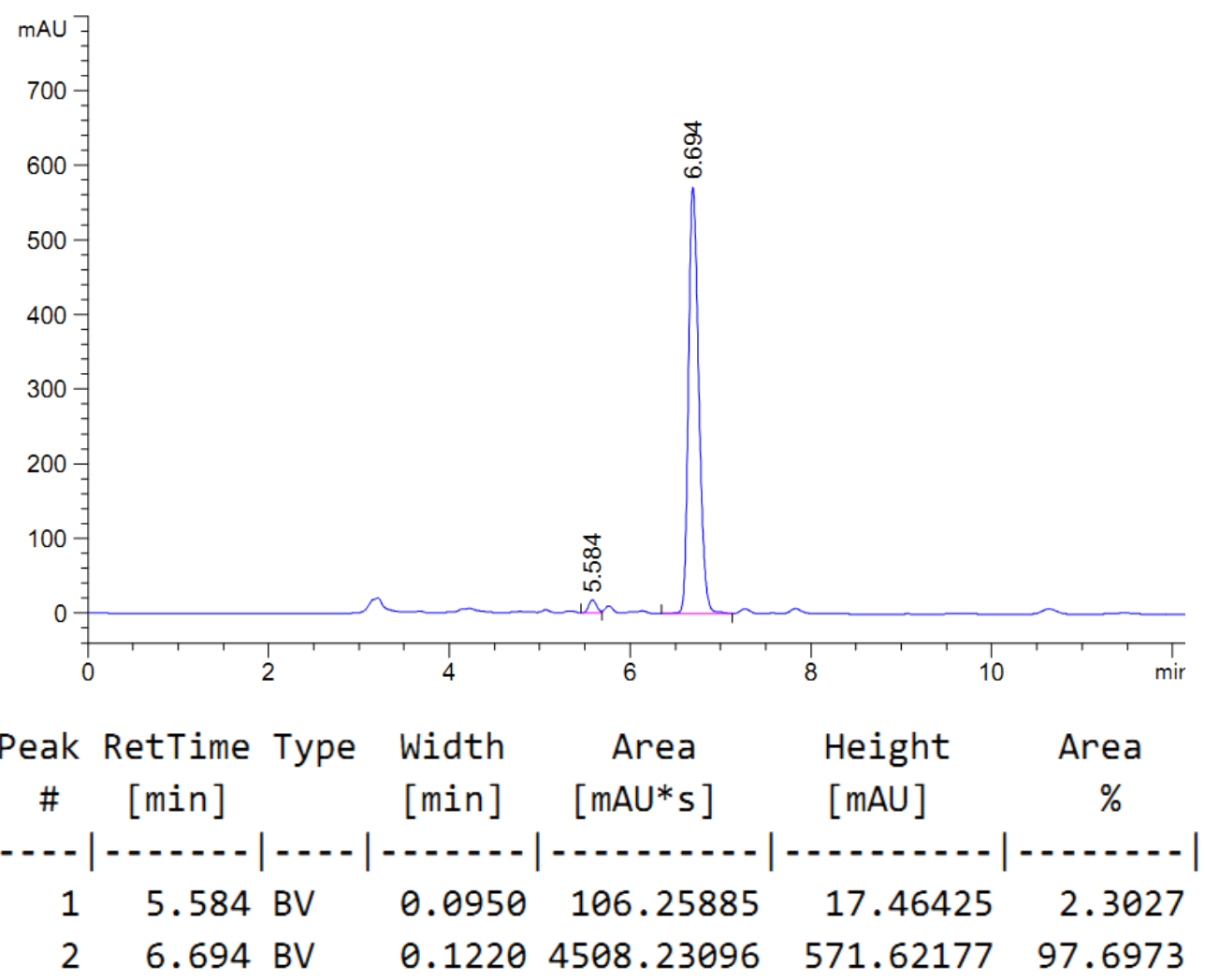

$2 q^{1} \mathrm{H}$

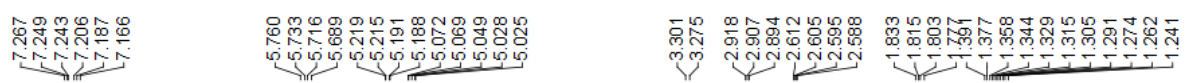

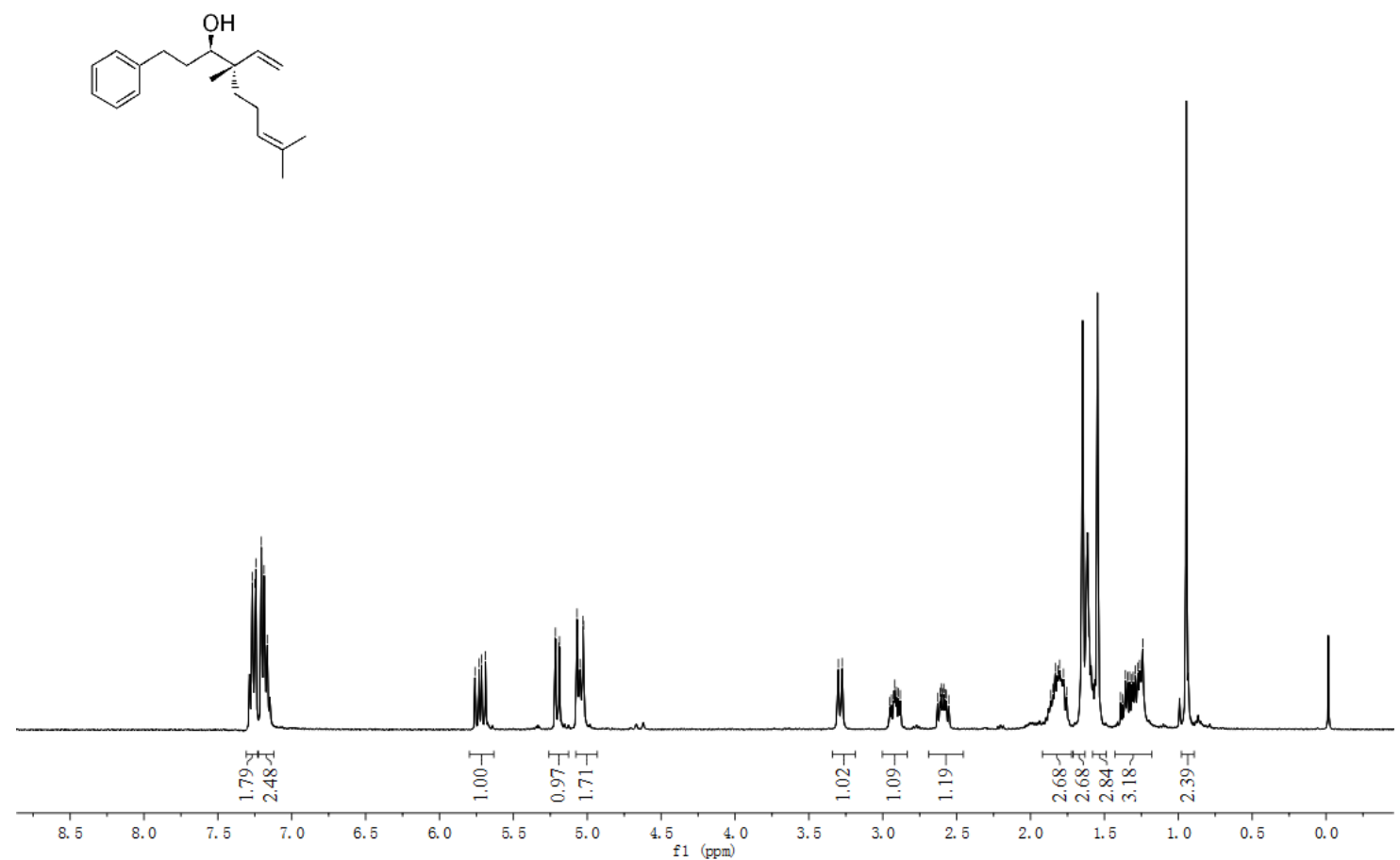


$2 q^{13} \mathrm{C}$
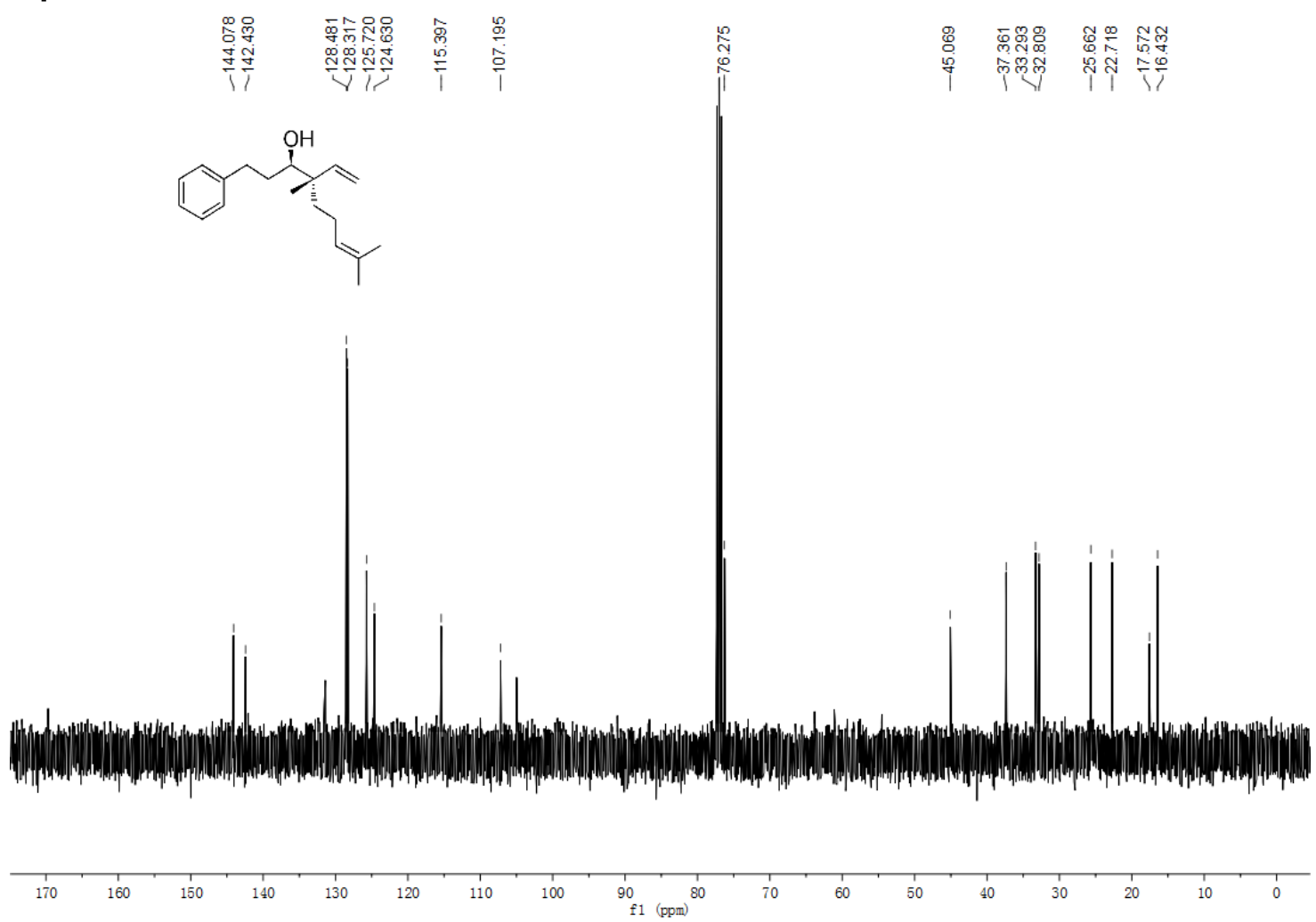

2q HPLC (racemic)

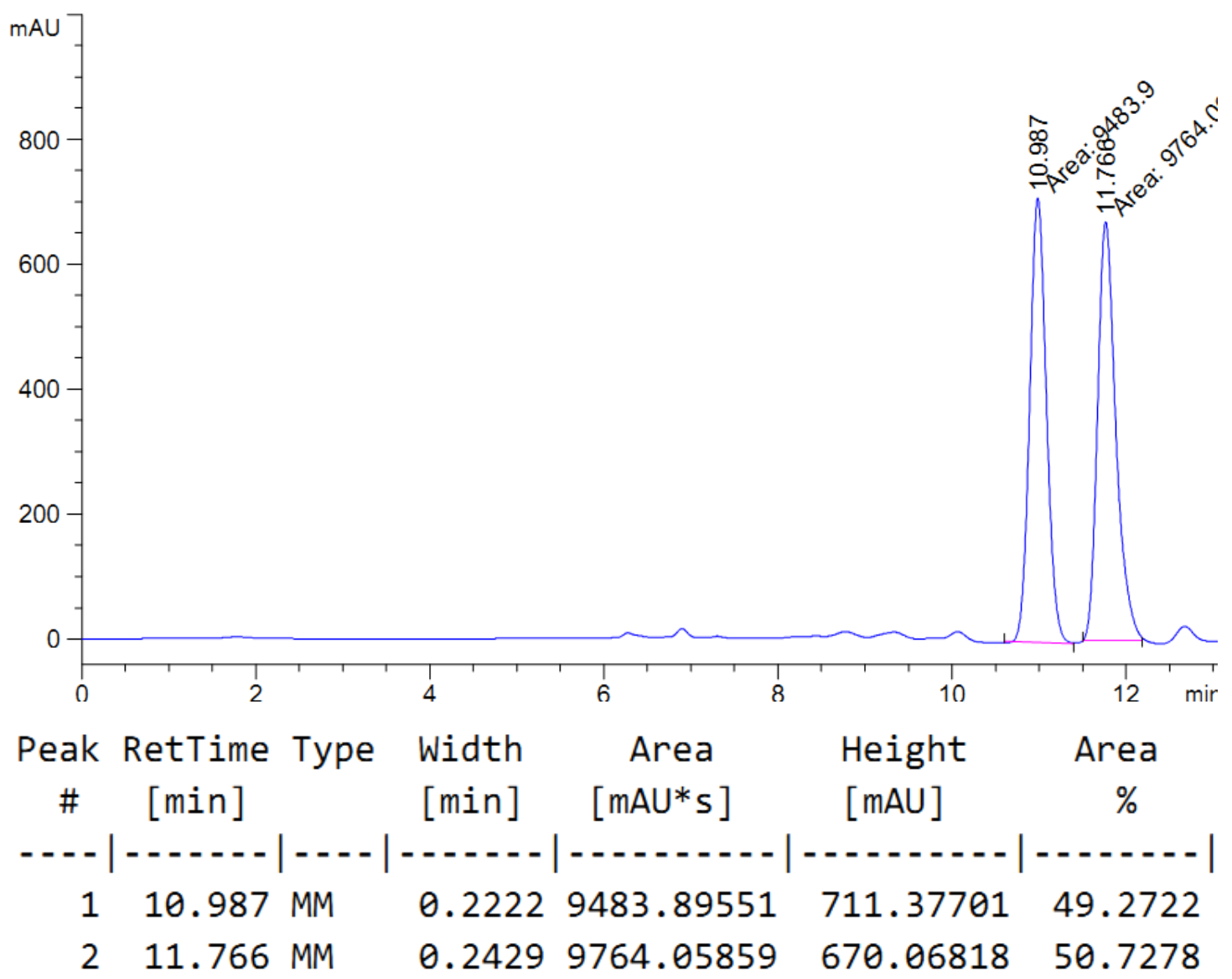


2q HPLC(95\% ee)
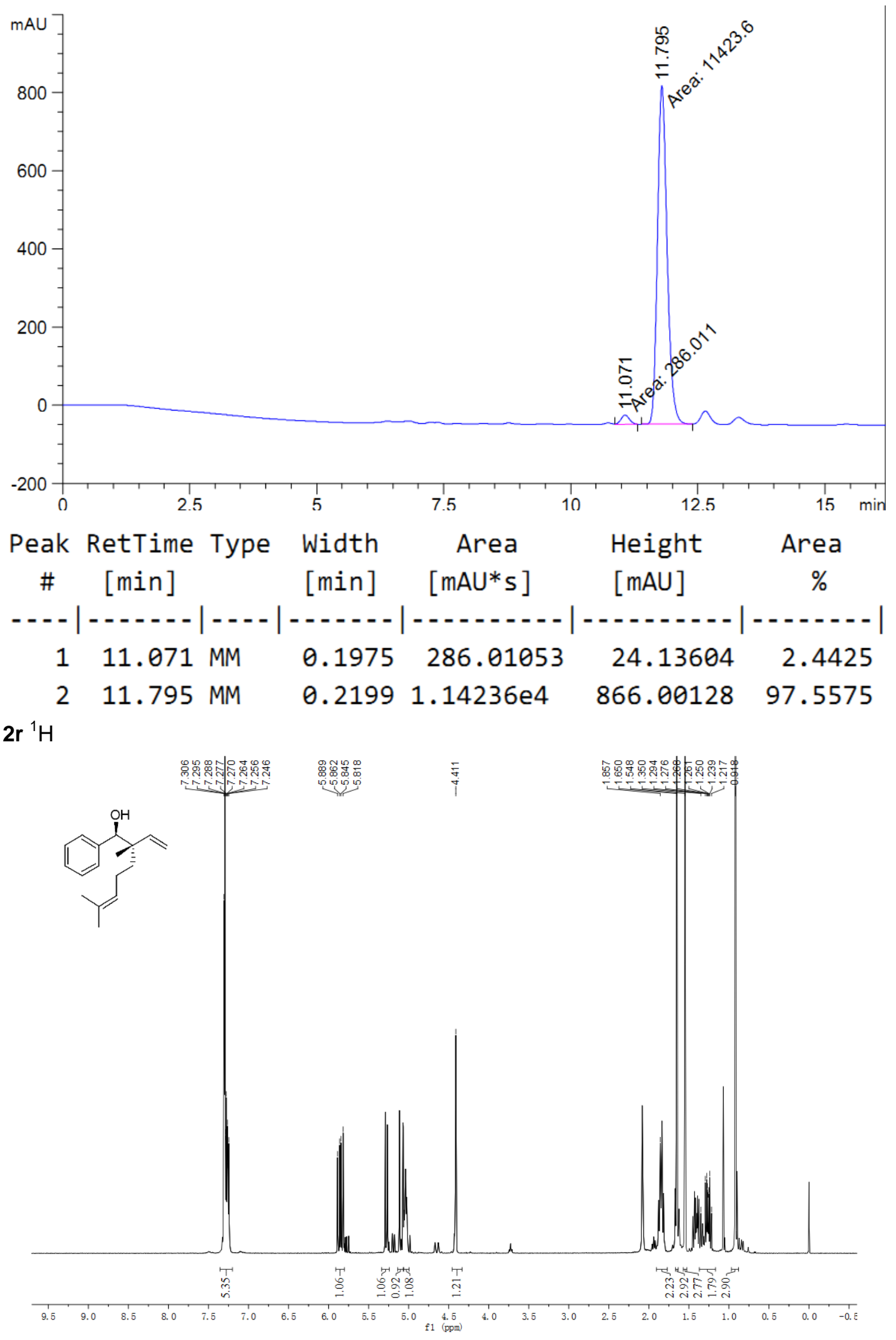
2r HPLC (racemic)

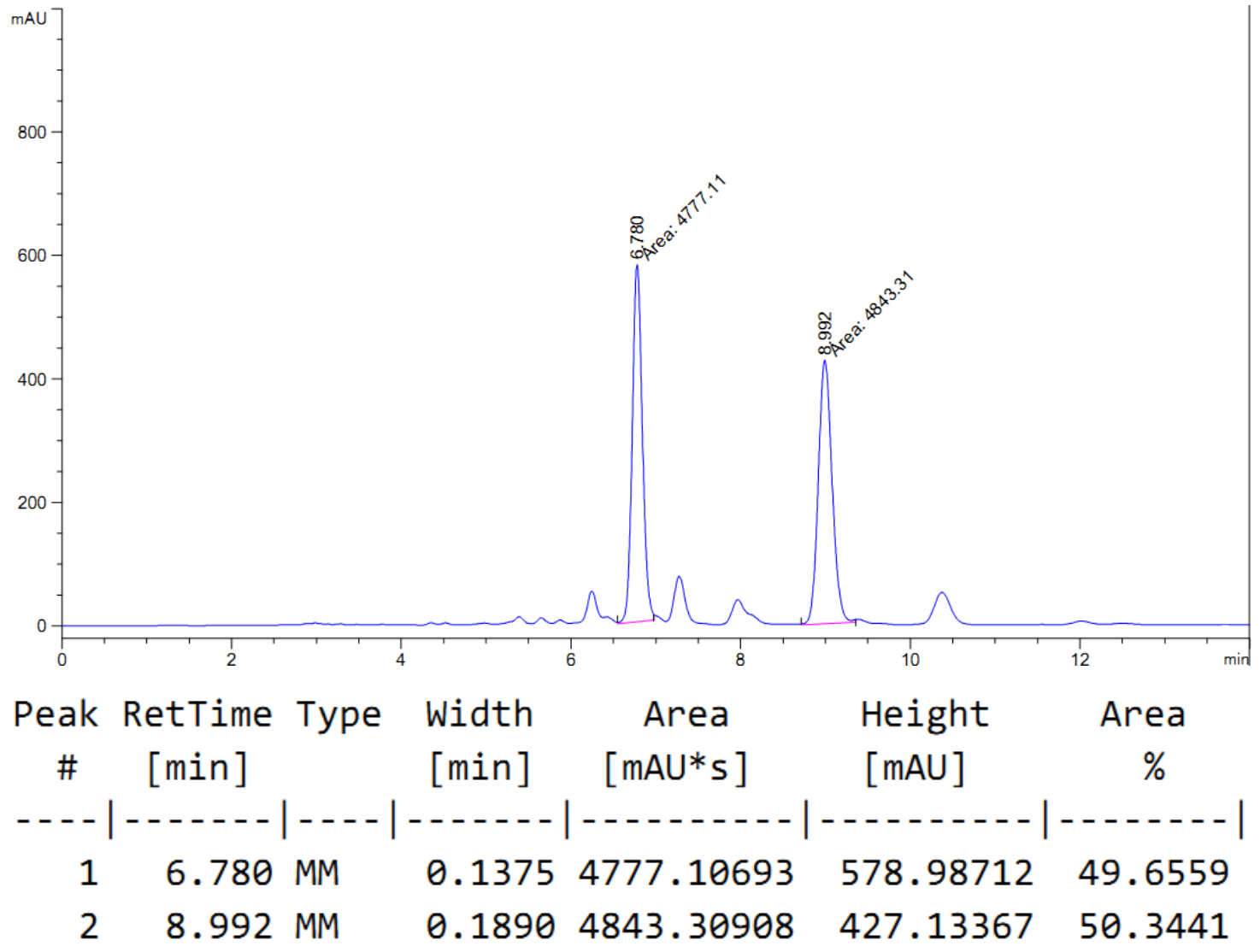

2r HPLC (93\% ee)

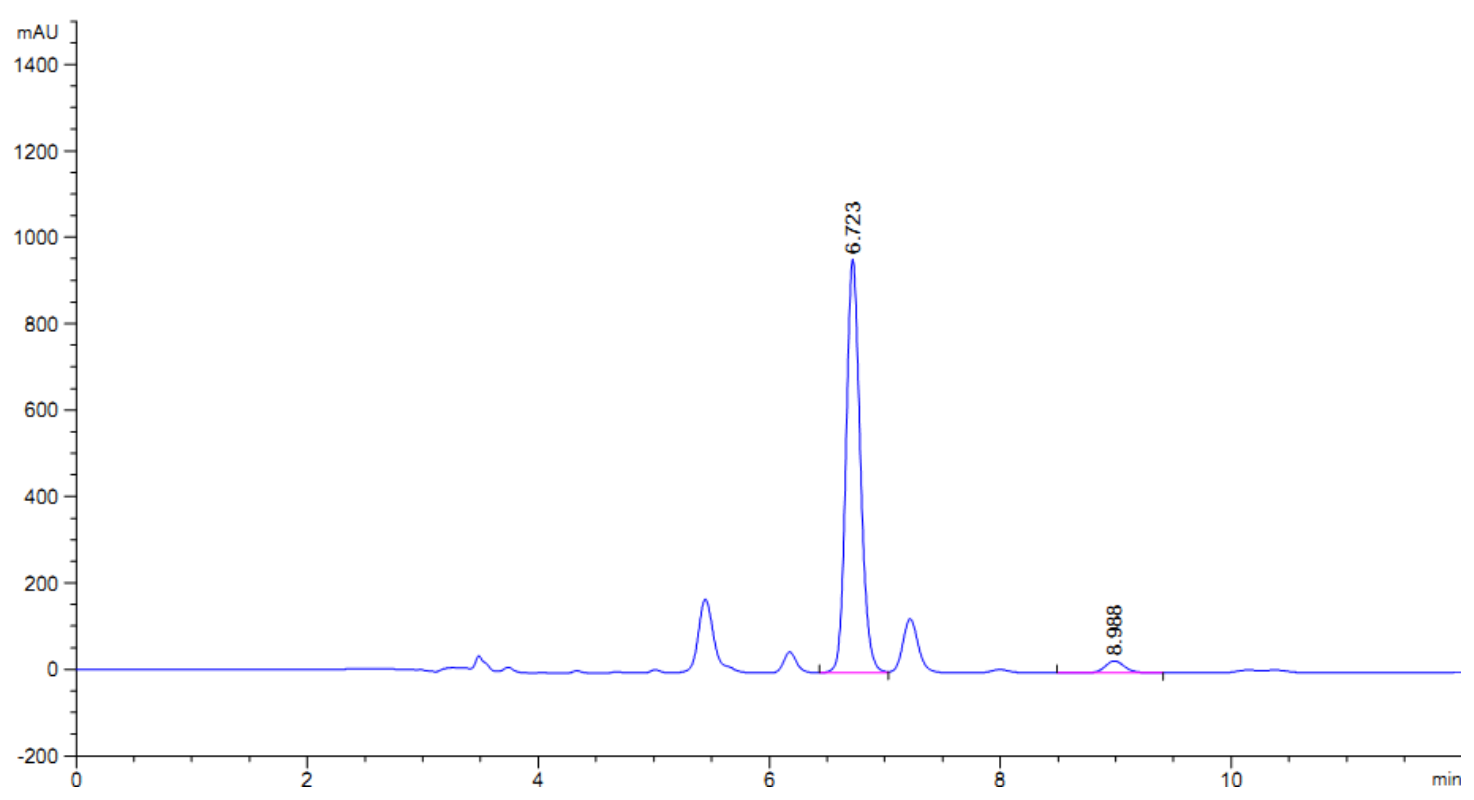




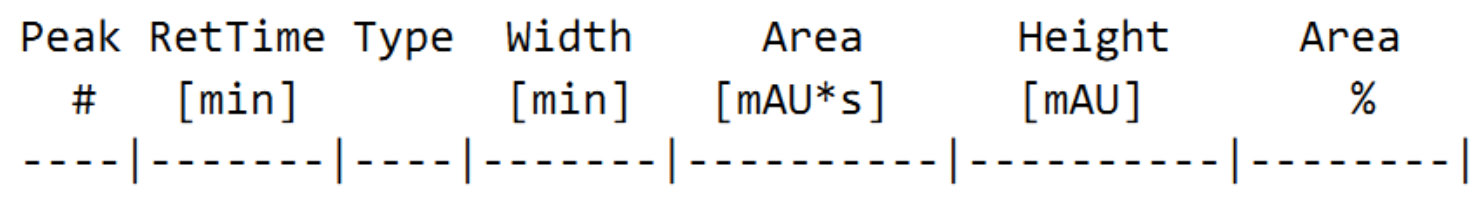
16.723 BV
$\begin{array}{llll}0.1323 & 8231.90137 & 957.27869 & 96.2067\end{array}$
$28.988 \mathrm{BB}$
$\begin{array}{lll}0.1799 & 324.57629 \quad 27.65638\end{array}$
3.7933

2s ${ }^{1} \mathrm{H}$

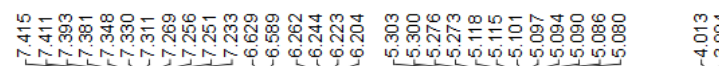

象
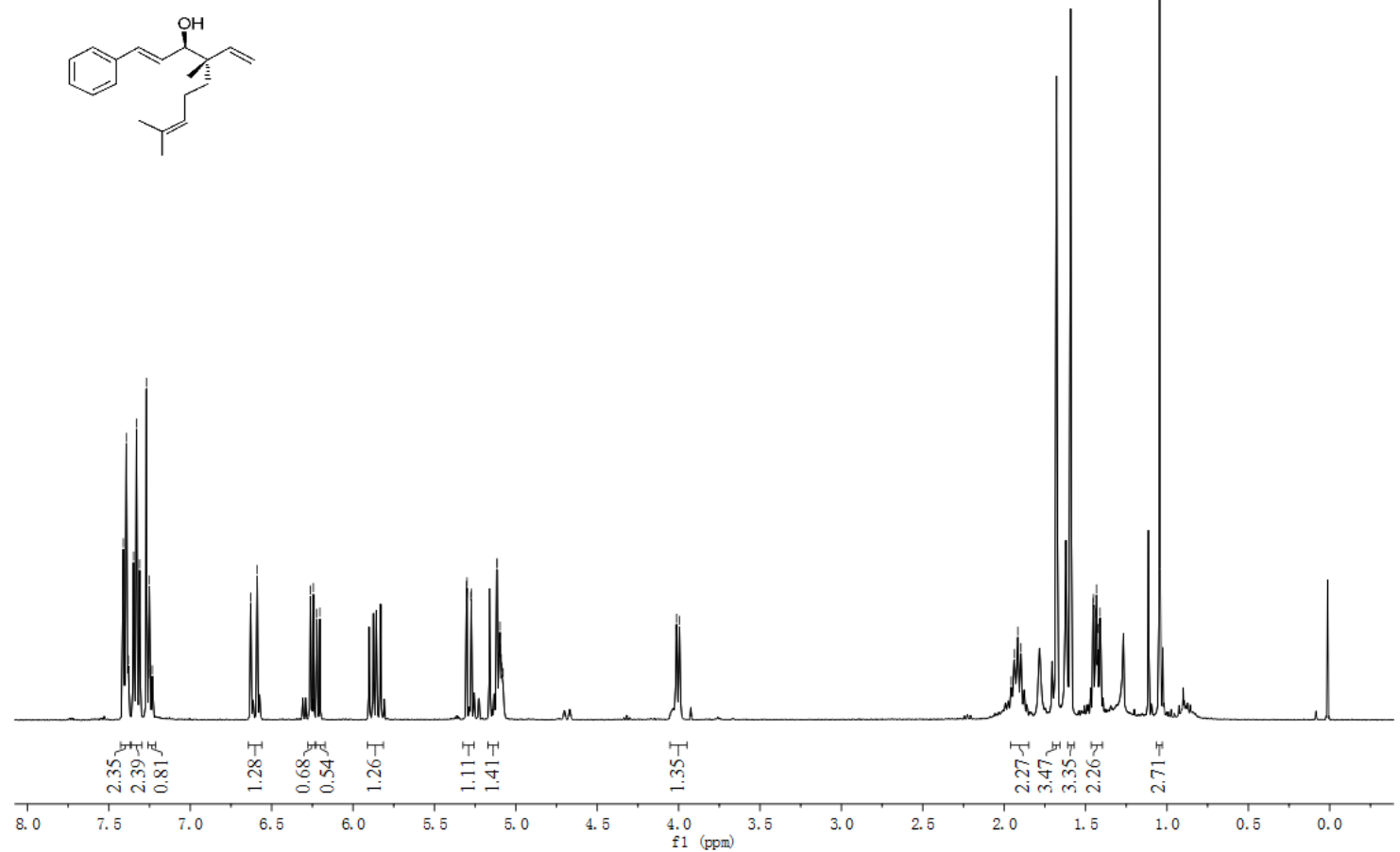

2s HPLC (racemic)

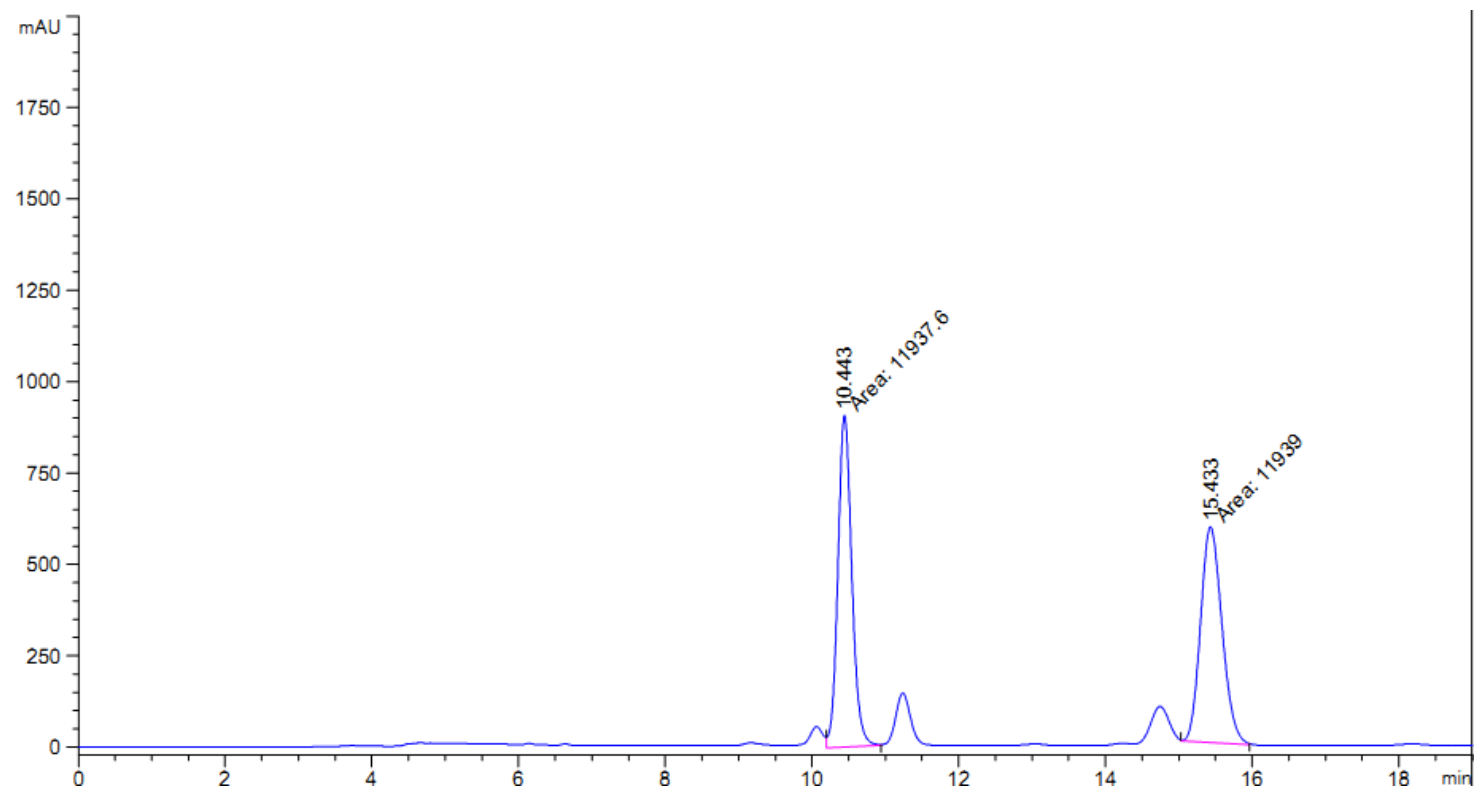




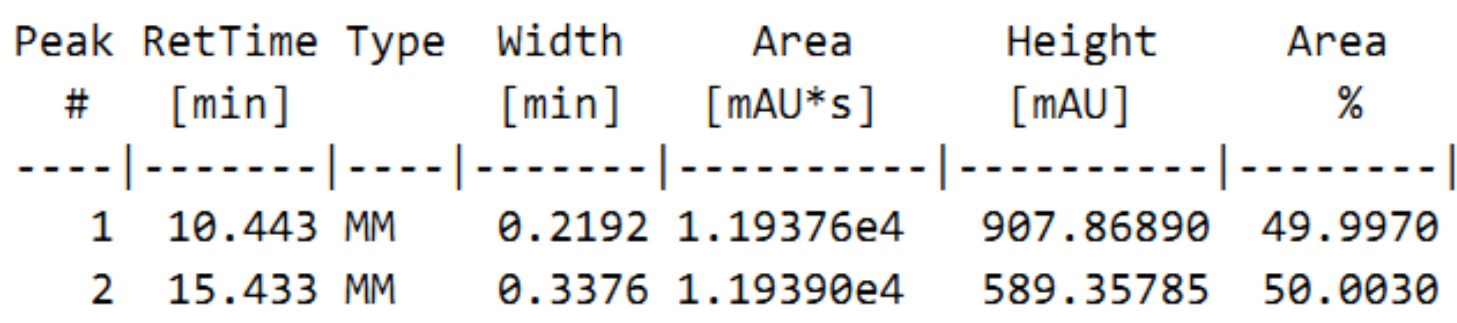

2s HPLC (90\% ee)

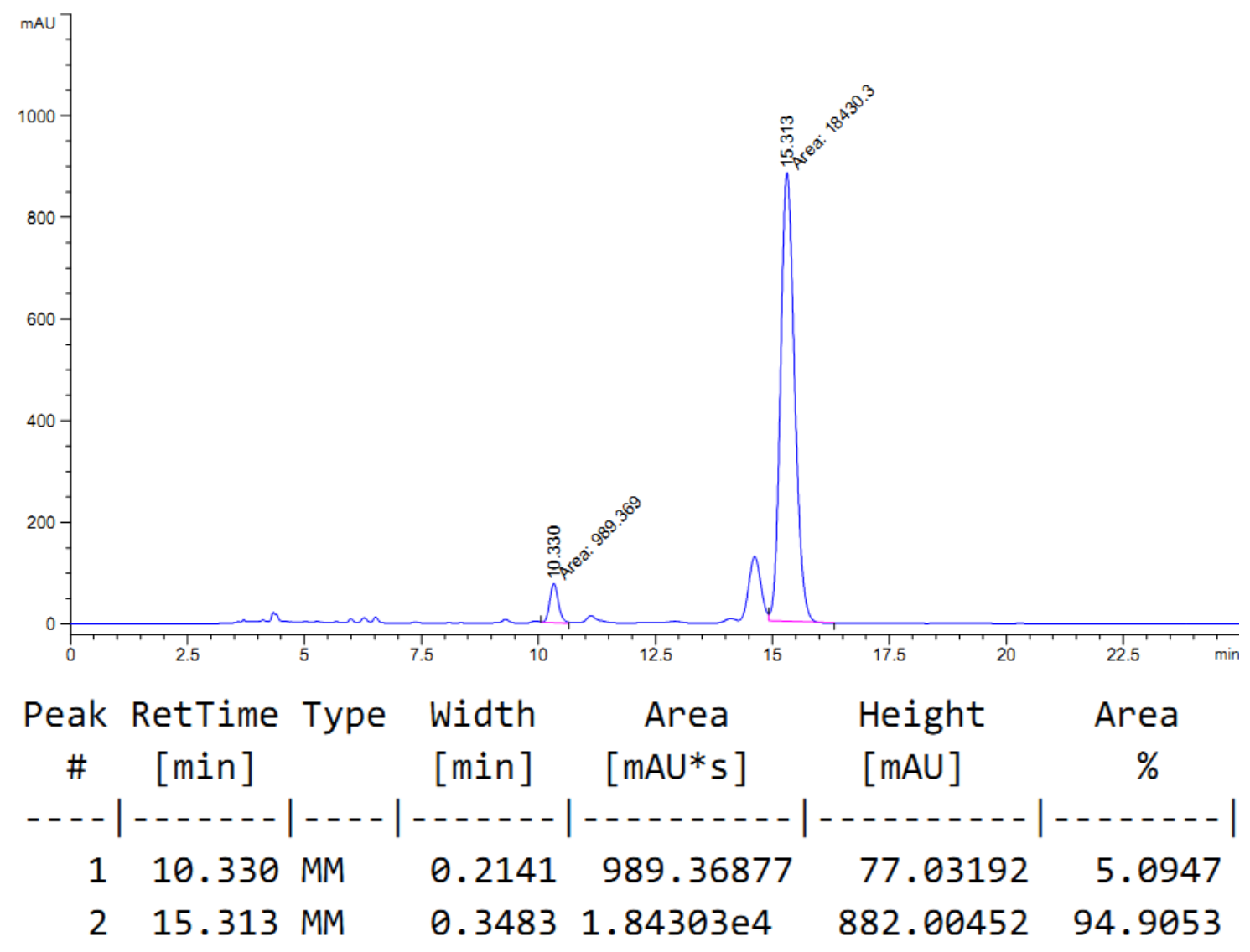


2t ${ }^{1} \mathrm{H}$

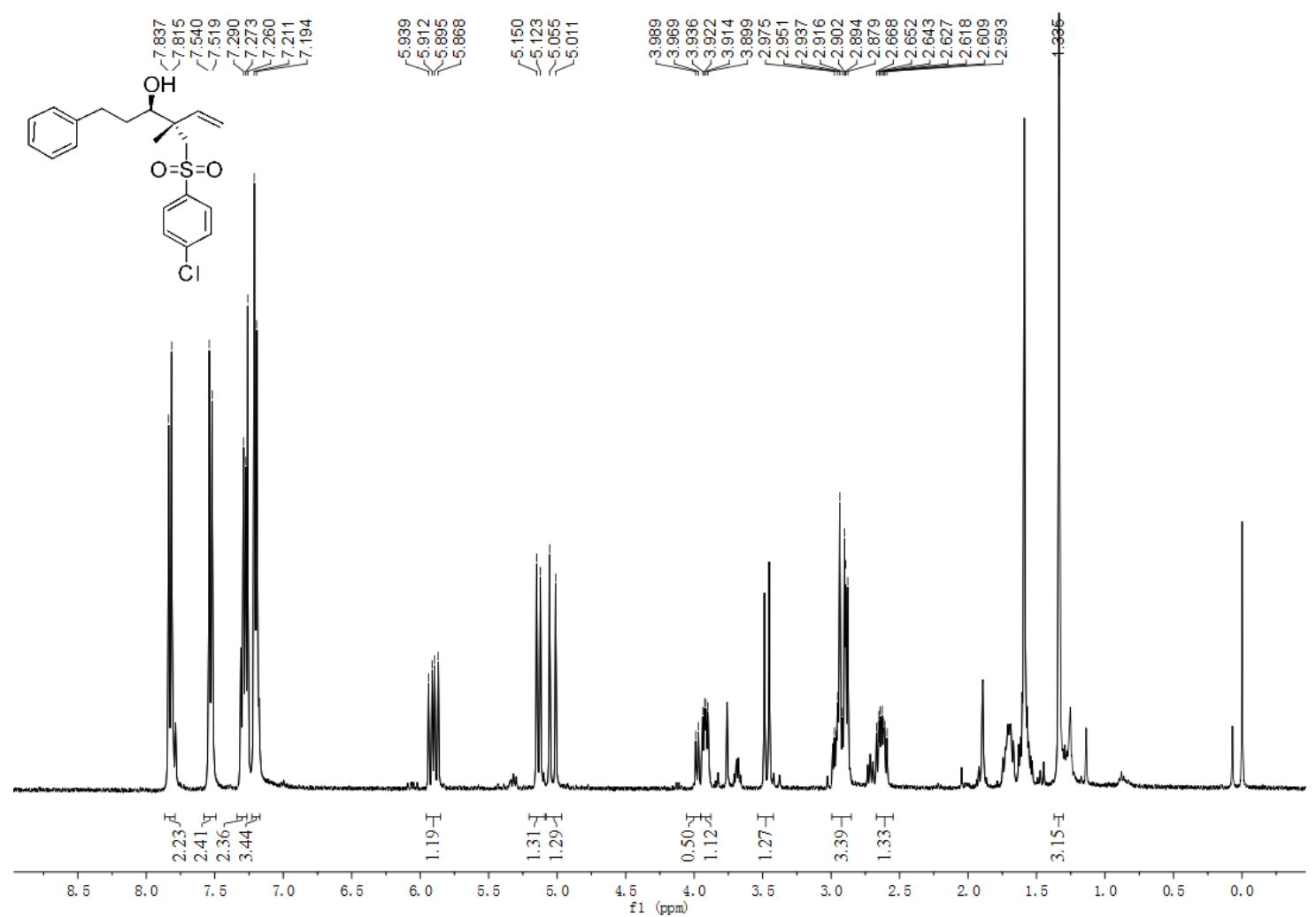

$2{ }^{13} \mathrm{C}$
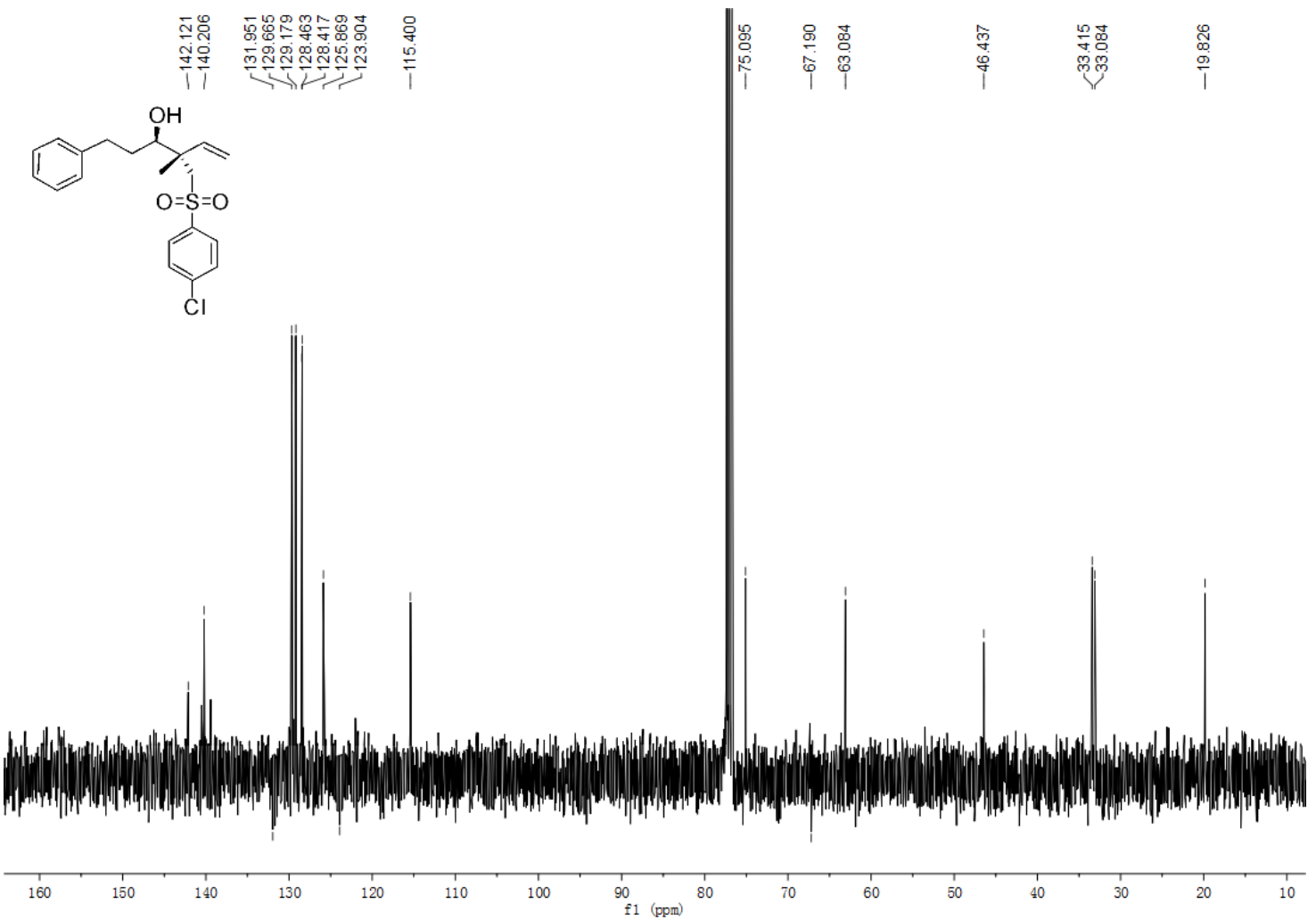
2t HPLC (racemic)

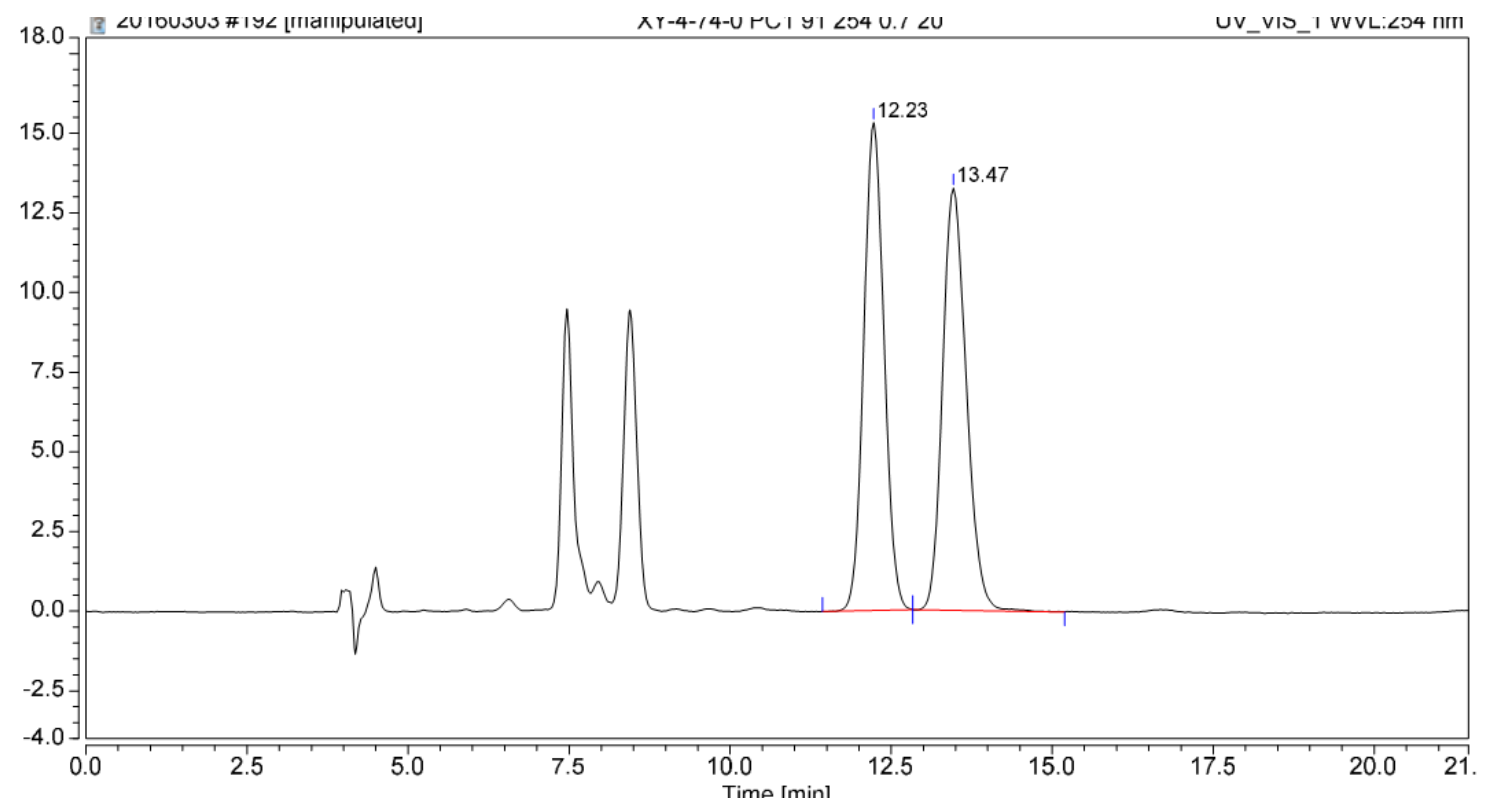

|lntegration Results

\begin{tabular}{|l|c|c|c|c|}
\hline No. & $\begin{array}{c}\text { Retention Time } \\
\min \end{array}$ & $\begin{array}{c}\text { Area } \\
\text { mAU*min }\end{array}$ & $\begin{array}{c}\text { Height } \\
\mathrm{mAU}\end{array}$ & $\begin{array}{c}\text { Relative Area } \\
\%\end{array}$ \\
\hline 1 & 12.227 & 5.588 & 15.312 & 49.69 \\
2 & 13.467 & 5.658 & 13.249 & 50.31 \\
\hline \multicolumn{2}{|r|}{ Total: } & 11.246 & $\mathbf{2 8 . 5 6 2}$ & 100.00 \\
\hline
\end{tabular}

2t HPLC (92\% ee)

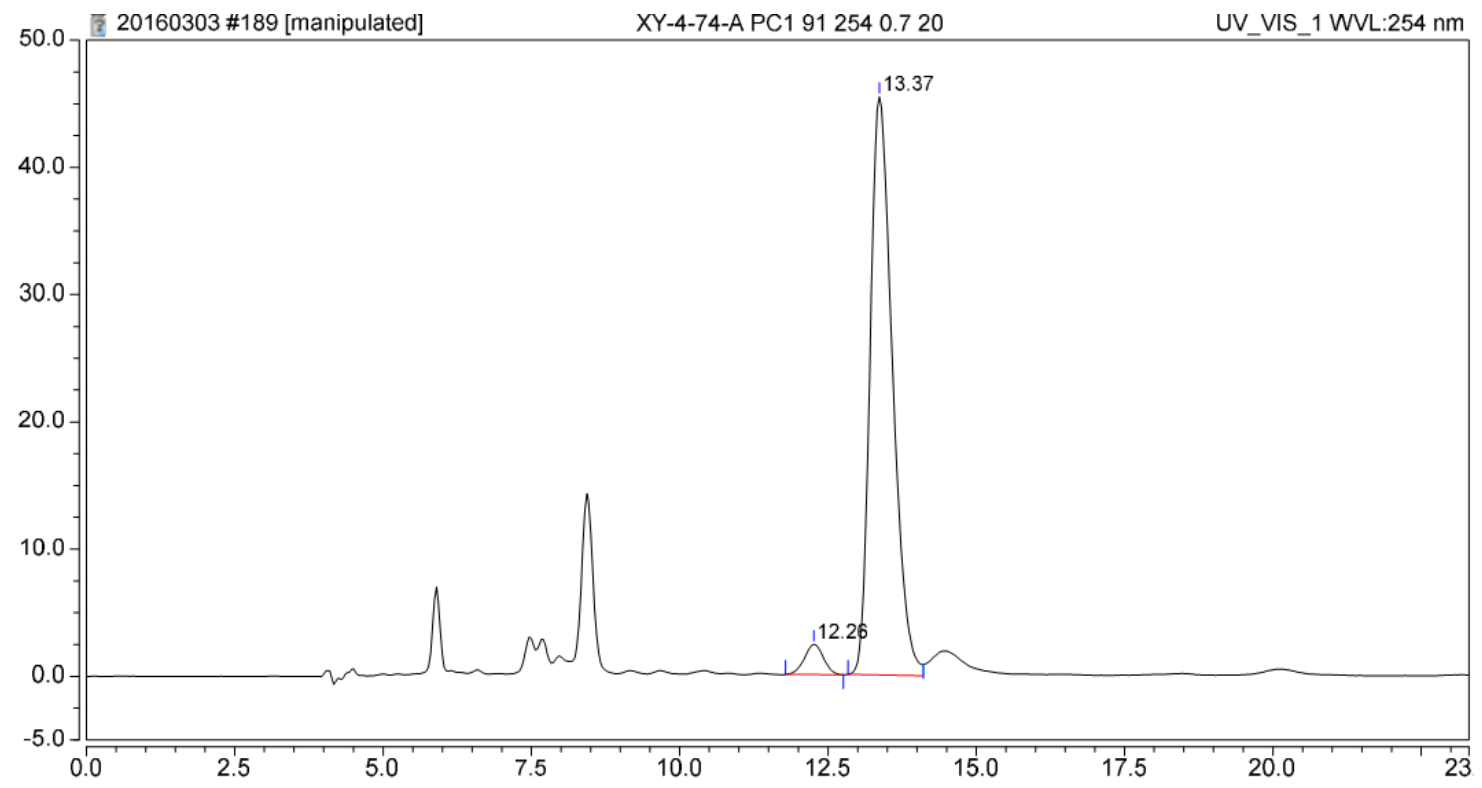

\begin{tabular}{|c|c|c|c|c|}
\hline \multicolumn{5}{|c|}{ Integration Results } \\
\hline No. & $\begin{array}{c}\text { Retention Time } \\
\text { min }\end{array}$ & $\begin{array}{c}\text { Area } \\
\mathrm{mAU* \operatorname {min }}\end{array}$ & $\begin{array}{c}\text { Height } \\
\text { mAU }\end{array}$ & $\begin{array}{c}\text { Relative Area } \\
\% \\
\end{array}$ \\
\hline 1 & 12.260 & 0.882 & 2.384 & 4.25 \\
\hline 2 & 13.367 & 19.864 & 45.457 & 95.75 \\
\hline \multicolumn{2}{|c|}{ Total: } & 20.745 & 47.841 & 100.00 \\
\hline
\end{tabular}


$2 u^{1} \mathrm{H}$

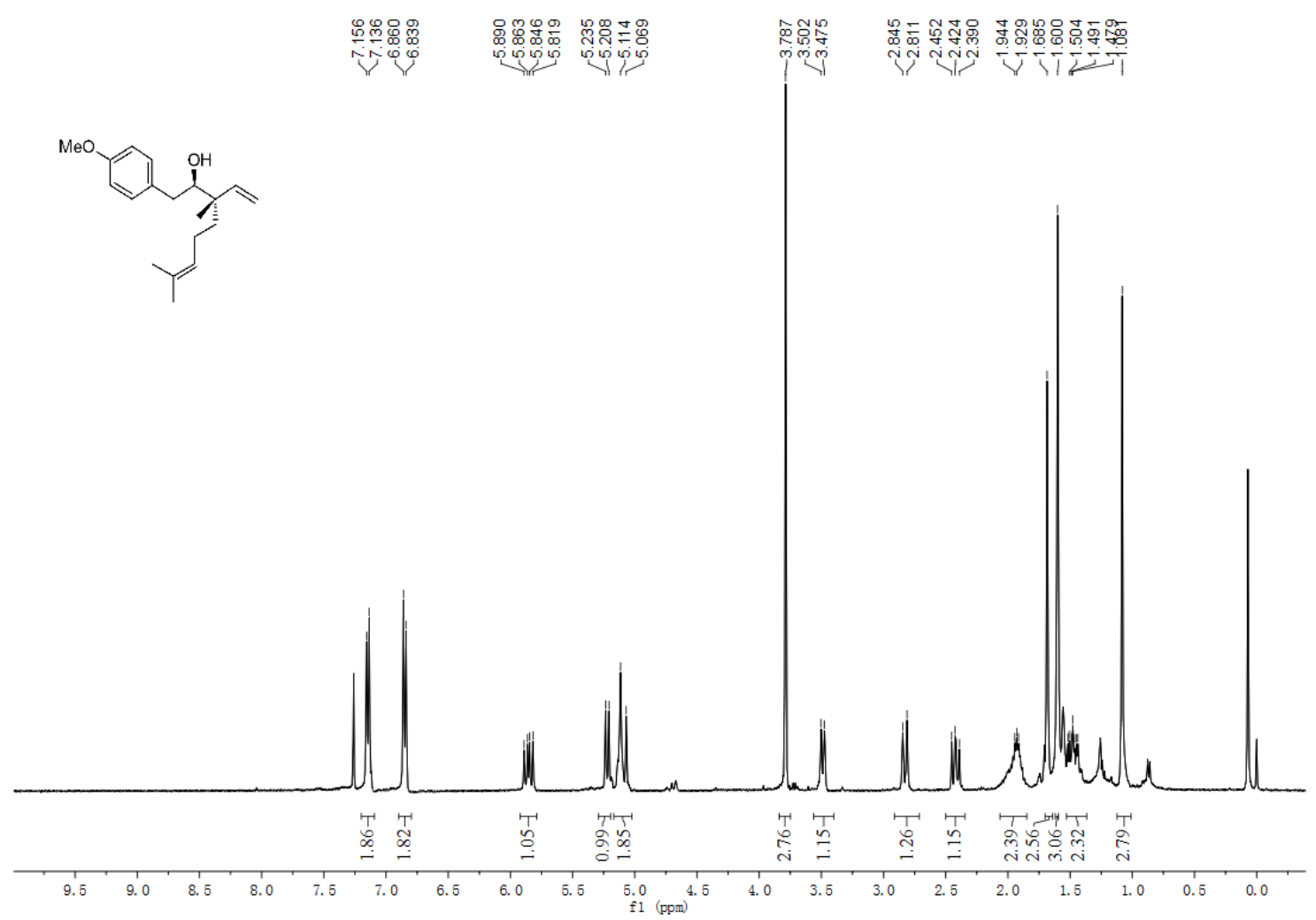

2u HPLC (racemic)
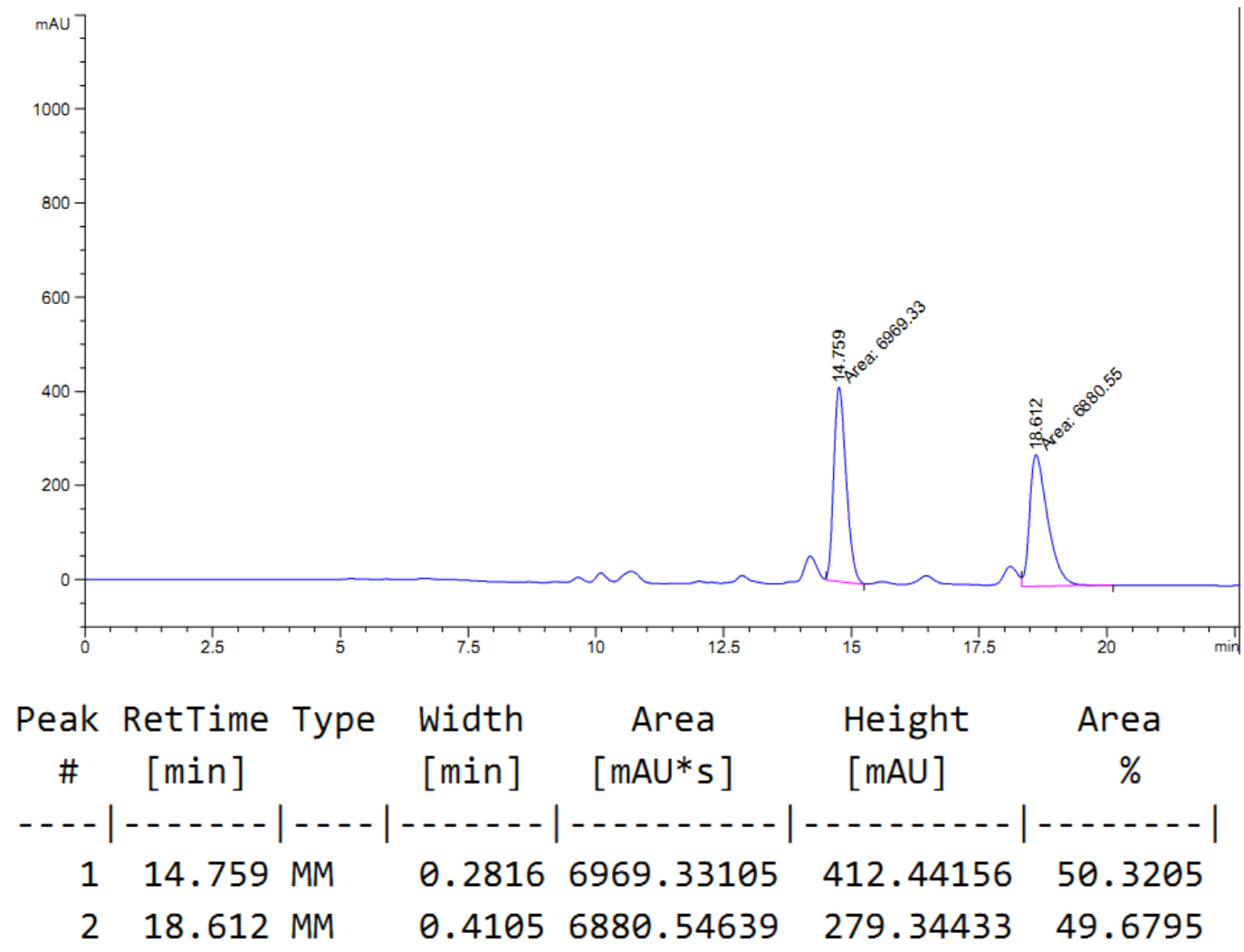

2u HPLC (91\% ee) 


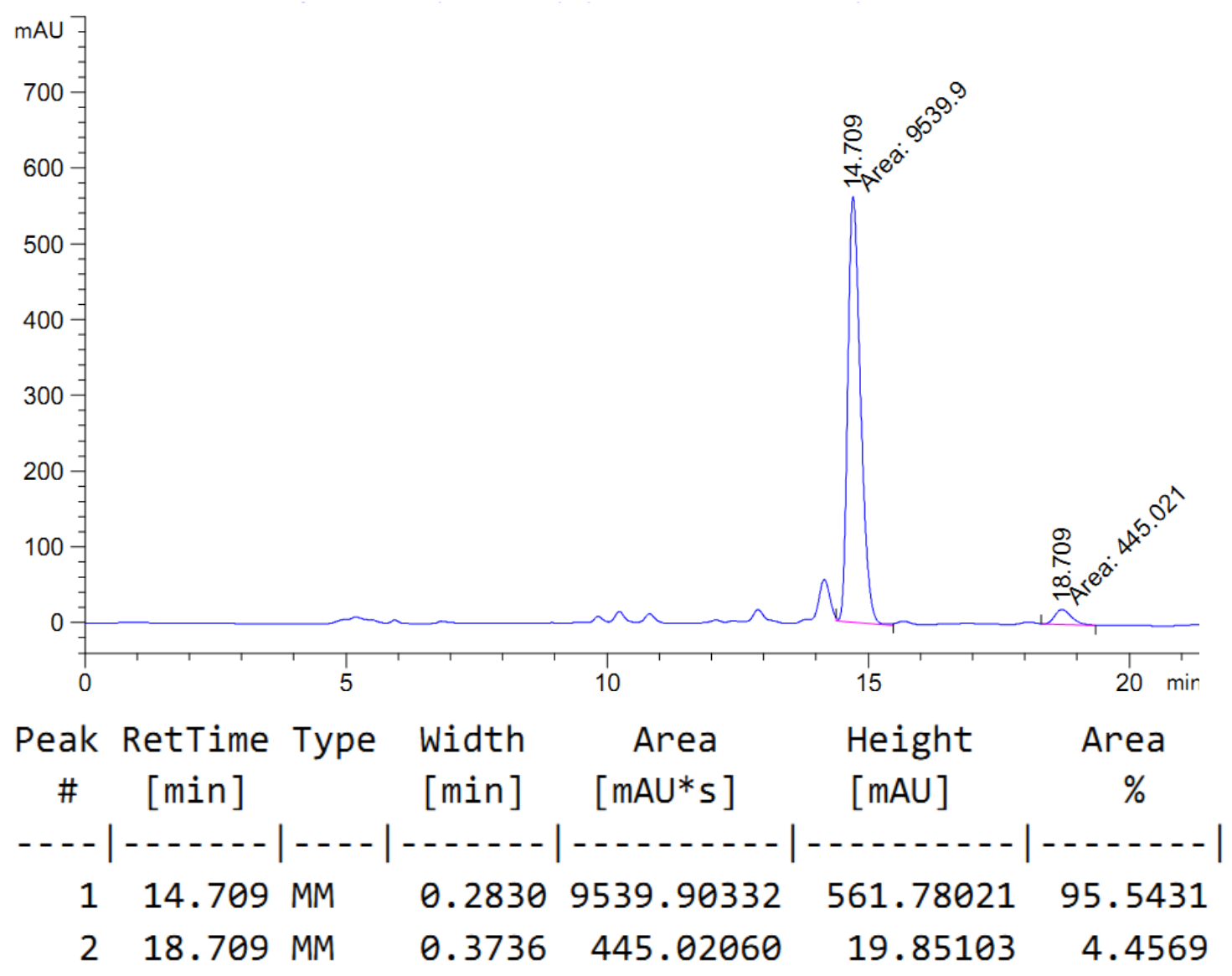

(1S,2S)-1-cyclohexyl-2-methyl-2-phenylbutane-1,4-diol- ${ }^{1} \mathrm{H}$ (3)

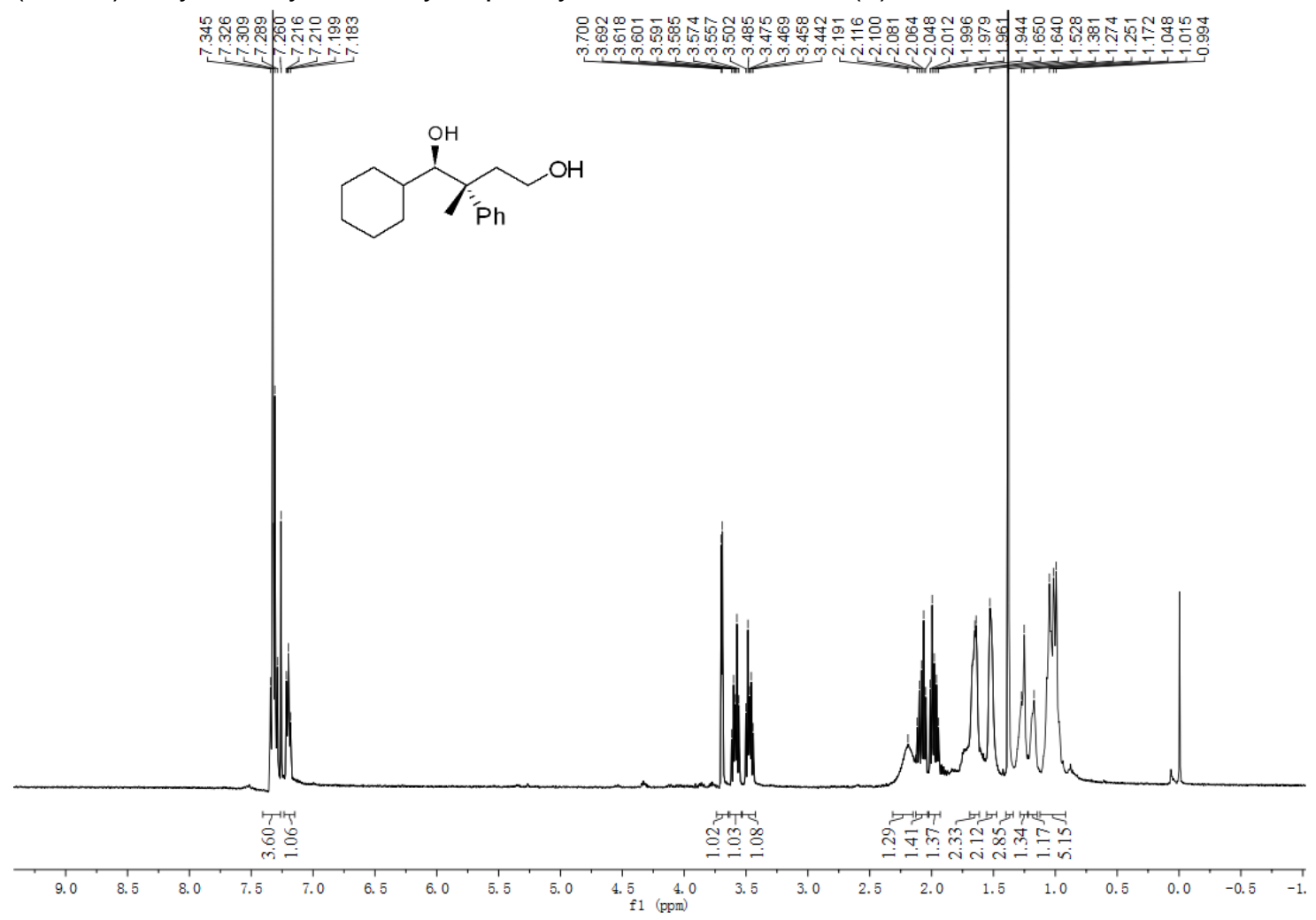


(S,E)-1-(3,7-dimethyl-3-vinylocta-1,6-dien-1-yl)-4-methoxybenzene- ${ }^{1} \mathrm{H}$

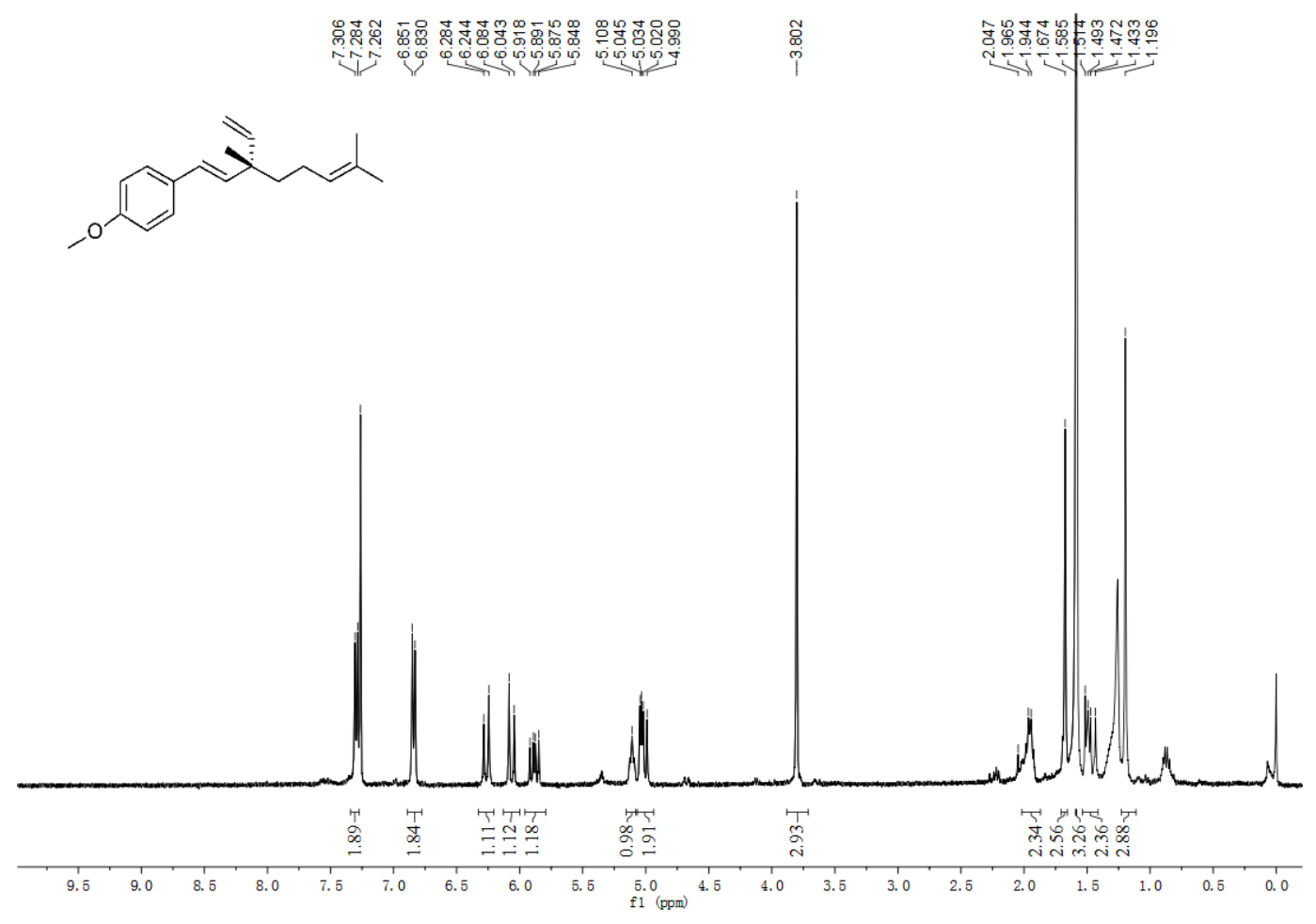

(S,E)-4-(3,7-dimethyl-3-vinylocta-1,6-dien-1-yl)phenol ((S)-Bakuchiol) (4) - ${ }^{1} \mathrm{H}$

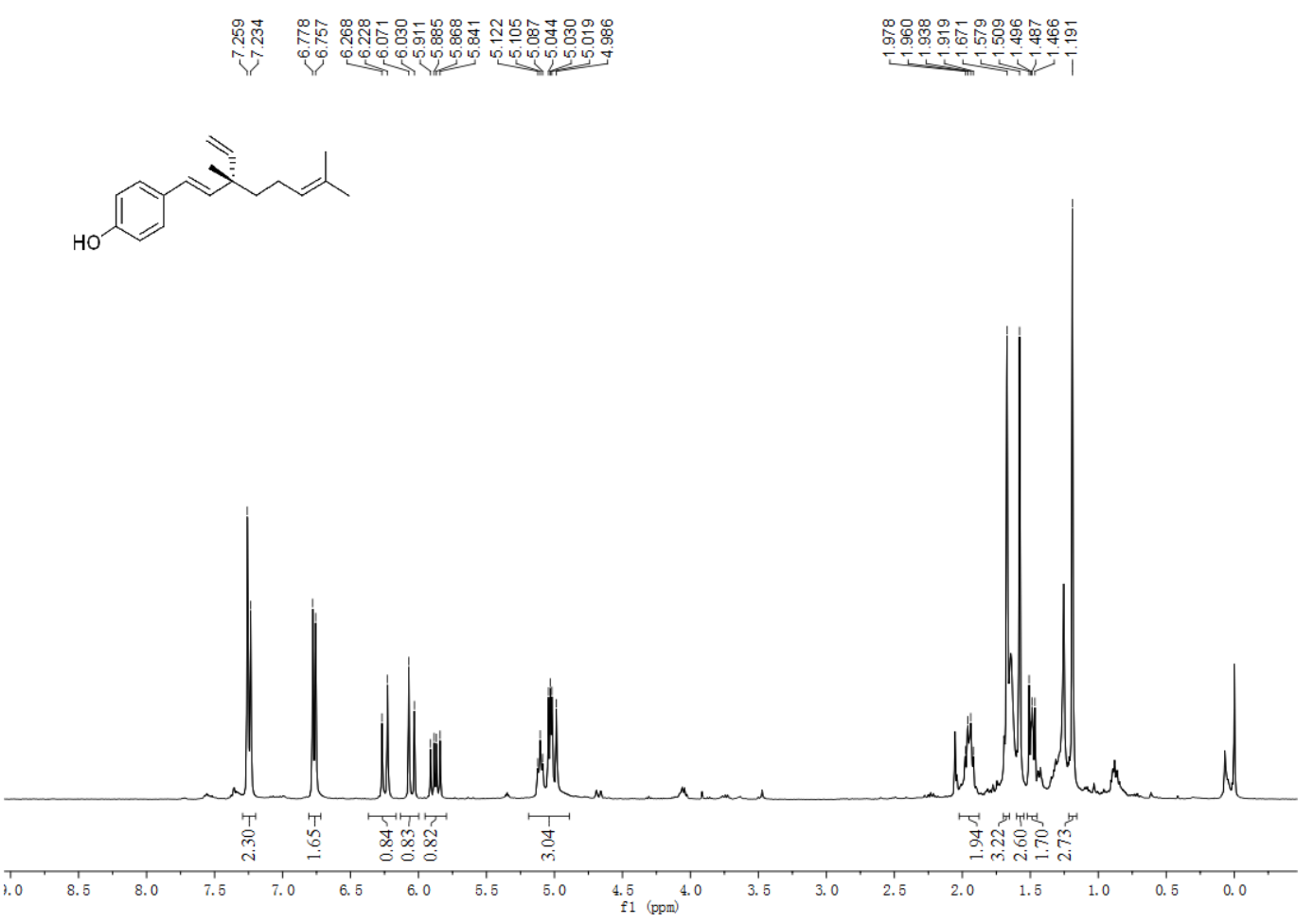


(S,E)-4-(3,7-dimethyl-3-vinylocta-1,6-dien-1-yl)phenol ((S)-Bakuchiol) $(5)-{ }^{13} \mathrm{C}$
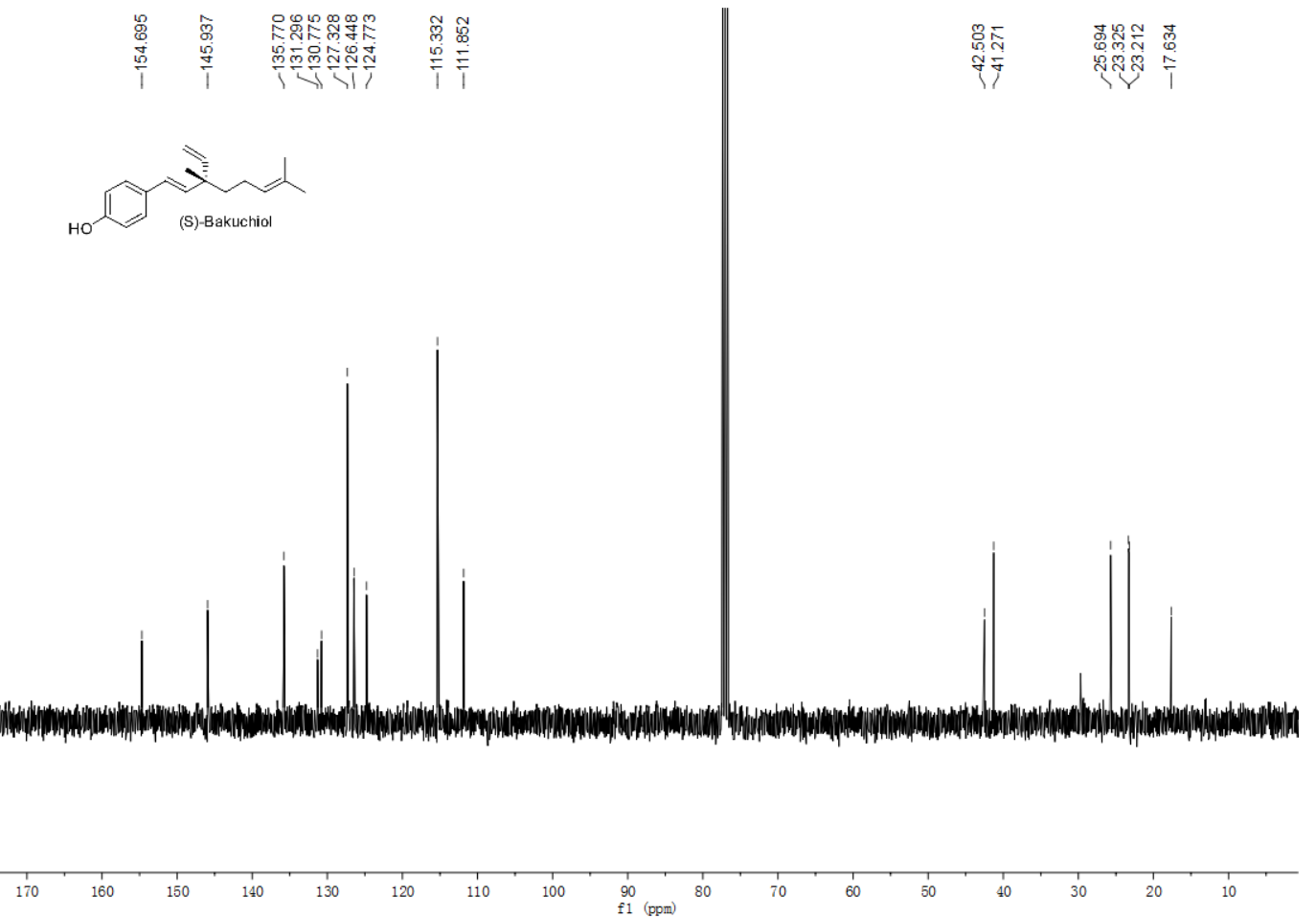